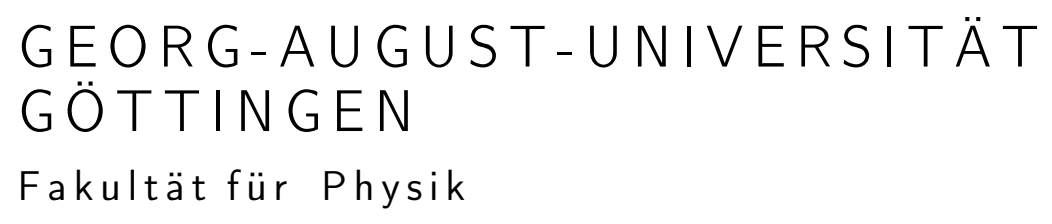

\title{
Multivariate Korrektur des Temperatureinflusses in der NIR-spektroskopischen Materialfeuchtebestimmung
}

\author{
DISSERTATION \\ zur Erlangung des Doktorgrades \\ der Mathematisch-Naturwissenschaftlichen Fakultäten \\ der Georg-August-Universität zu Göttingen
}

vorgelegt von:

Sven Groß

aus Göttingen

Göttingen 2009 
D7

Referent: Prof. Dr. Werner Lauterborn

Korreferent: Prof. Dr. Gerd Marowsky

Tag der mündlichen Prüfung: 29. April 2009 


\section{Inhaltsverzeichnis}

1 Einleitung 1

2 Grundlagen der Materialfeuchtebestimmung 3

2.1 Übersicht der Techniken und Methoden zur Bestimmung der Materialfeuchte . . . . . . . . . . . . . . . . 4

2.2 Unterschied Gesamt- und wasserselektive Materialfeuchte . . . . . . 5

2.3 Feuchte in festen und flüssigen Stoffen . . . . . . . . . . . . 6

2.4 Die chemische Verbindung Wasser . . . . . . . . . . . . . . . . . 9

2.5 Spektroskopische Eigenschaften des Wassermoleküls . . . . . . . . . 12

2.5.1 Der harmonische Oszillator . . . . . . . . . . . . . . . . . 12

2.5.2 Der anharmonische Oszillator . . . . . . . . . . . . 15

2.5.3 Normalschwingungen des Wassermoleküls . . . . . . . . . . 17

2.5.4 Normalschwingungen des Wassermoleküls in der kondensierten Phase, flüssig oder in einer Feststoffmatrix . . . . . . . . 19

2.5.5 Oberton- und Kombinationsschwingungen . . . . . . . . . 21

3 Experimentelle Techniken 25

3.1 Referenzanalytik . . . . . . . . . . . . . . . . 25

3.1.1 Gesamtfeuchtebestimmung nach dem Prinzip der Thermogravimetrie . . . . . . . . . . . . . . . 26 26

3.1.2 Wassergehaltsbestimmung nach dem Prinzip der Coulometrie. 28

3.2 Spektroskopische Methoden . . . . . . . . . . . . . . . . . 32

$3.2 .1 \quad$ NIR-Reflexionsspektroskopie . . . . . . . . . . . . . . . . 33

3.3 Beschreibung des Versuchsaufbaus zur NIR-Spektroskopie . . . . . . . 35

3.3.1 Aufbau zur Transmissionsmessung an Wasser . . . . . . . . 35

3.3.2 Aufbau zur Reflexionsmessung an Pulvern bei konstanter Materialfeuchte und variierender Temperatur . . . . . . . . 38

3.4 Auswahl der Modellsubstanz . . . . . . . . . . . . . . . . . . . . . 42

3.5 Datenaufnahme, Speicherung und Verarbeitung . . . . . . . . . . 43

3.6 Probenvorbereitung . . . . . . . . . . . . . . . . . . . . . . . 44

4 Methoden zur Datenauswertung 49

4.1 Chemometrie und multivariate Statistik . . . . . . . . . . . . . . 49

4.1.1 Partial Least Squares Regression - PLS . . . . . . . . . . . 49 
4.1.2 Bewertung der Güte einer Kalibrierung . . . . . . . . . . . . . 53

4.1.3 Ausreißererkennung . . . . . . . . . . . . . . . 56

4.2 Datenvorbehandlung und -verarbeitung . . . . . . . . . . . 65

4.2.1 Korrektur konstanten Basislinienversatzes . . . . . . . . . . 65

4.2.2 Min-Max-Normierung . . . . . . . . . . . . . . . 66

4.2.3 Multiplikative Streukorrektur (MSC) . . . . . . . . . . . . . 67

4.2.4 Standard Normal Variate (SNV) . . . . . . . . . . . . 68

4.2.5 Savitzky-Golay-Glättung und Ableitung . . . . . . . . . . . 69

4.3 Entwicklung chemometrischer Methoden . . . . . . . . . . . . . 71

4.3.1 Design eines Kalibrierdatensatzes . . . . . . . . . . . . . . . 71

4.4 Piecewise Direct Standardization (PDS) . . . . . . . . . . . . . . . 72

5 Experimentelle Ergebnisse 75

5.1 Quereinflüsse . . . . . . . . . . . . . . . . . . 75

5.1 Partikelgröße . . . . . . . . . . . . . . 76

5.1.2 Verfärbungen der Probe . . . . . . . . . . . . . . . 78

5.1.3 Abstand Probe - Sensorkopf . . . . . . . . . . . . . . 79

5.2 Einfluss der Probentemperatur auf die Vorhersagen . . . . . . . . . . 81

5.3 Veränderung der Kalibriermodellkenngrößen aufgrund der Temperaturvarianz . . . . . . . . . . . . . . . 83

5.4 Zusammenfassung der experimentellen Ergebnisse . . . . . . . . . . 85

6 Diskussion 89

6.1 Verhalten der Vorhersage bei variierender Temperatur . . . . . . . . . 89

6.1.1 Zusammenhang Vorhersagefehler und Steigung der linearen Regression . . . . . . . . . . . . . . . 89

6.1.2 Zusammenhang Steigung der linearen Regression und Temperaturvarianz . . . . . . . . . . . . . . . . 90 90

6.2 Temperaturkorrektur im Referenzwertraum . . . . . . . . . . . . . 94

6.3 Temperaturkorrektur über Dotierung des Kalibrierdatensatzes . . . . 96

6.4 Ansatz durch Kalibriertransfer des Spektraldatensatzes . . . . . . . . 98

6.5 Fazit der Diskussion . . . . . . . . . . . . . . . . . . . . 102

7 Methode zur Durchführung der Temperaturkorrektur 103

8 Zusammenfassung 109

9 Ausblick 111

A Probennachweis und Temperaturmesswerte $\quad 115$

A.1 Herkunftsnachweis der verwendeten Mehlproben . . . . . . . . . . . . 115

A.2 Temperaturmesswerte und Einstellungen des Thermostaten . . . . . . 116 
Abbildungsverzeichnis

Literaturverzeichnis 



\section{Kapitel 1}

\section{Einleitung}

Die Materialfeuchte spielt in vielen Bereichen des täglichen Lebens eine wichtige Rolle. Nicht zuletzt die Qualität eines Produktes (z. B. Lebensmittelindustrie, Pharmazie, chemische Industrie) wird von dem Gehalt an flüchtigen Bestandteilen oft maßgeblich beeinflusst. Mit der Automatisierung von Produktionsprozessen ist in der Regel der Antrieb zu einer höheren Ausbeute und Effizienzsteigerung verbunden. Außerdem ist der Bedarf an sicheren Prozessen nicht zuletzt in Hinblick auf die Produktqualität sehr groß. Im Zuge der Automatisierung von Produktionsprozessen steigt daher der Bedarf an schnellen, zerstörungsfreien Methoden zur insitu Materialfeuchtebestimmung ständig. Die Anforderungen an diese Methoden sind jedoch oft von unterschiedlicher Natur, so dass der Bedarf nicht von einer Technologie oder Methode allein gedeckt werden kann. In vielen Prozessen ist eine offline Messung völlig ausreichend oder sogar vom Anwender aus Prozessgründen gewünscht, wobei hier meist der Wunsch nach möglichst schnellen offline Technologien besteht. Aber auch der Bedarf an online Messtechniken, zur direkten Steuerung und Regelung des Prozesses bei sehr kurzen Messzeiten ist keine Seltenheit mehr. Die entsprechende Technologie ist dabei von den Probeneigenschaften wie Korngröße oder Konsistenz abhängig.

Die optischen Messtechniken bieten hier eine Vielzahl an Möglichkeiten zur zerstörungsfreien und berührungslosen [1, 2, 3, 4, 5] Datenerfassung und Sensorik. Viele Parameter eines industriellen Prozesses können mit Hilfe spektroskopischer Methoden $[6,7,8,9]$ erfasst und ausgewertet werden. Spektroskopische Methoden bieten von der einfachen Einzelanalytenbestimmung [10, 11, 12] wie z. B. der Bestimmung des Wassergehaltes einer Probe bis hin zur Multikomponentenanalyse von gleichzeitiger Bestimmung mehrerer Analyten eine Vielzahl an Möglichkeiten der Messung [13, 14, 15]. Die Komponentenbestimmung mittels optischer Spektroskopie lässt sich besonders in Hinsicht auf die Bestimmung des Wassergehaltes einer Probe, der meist den Hauptteil der Materialfeuchte darstellt [16], im nahen Infrarot zwischen 1100 und $2500 \mathrm{~nm}$ [17] im Normalfall sogar im Bereich zwischen 950 und $1650 \mathrm{~nm}$ [18] realisieren. Die hierfür relevanten Banden der IR-aktiven OH-Schwingungen des Wassermoleküls sind relativ breit und zeigen ihr Absorptionsmaximum bei $1450 \mathrm{~nm}$ und $1930 \mathrm{~nm}$ [19]. Anhand der spektralen Struktur der Wasserabsorption können Aussa- 
gen z. B. über die Temperatur oder die Art des Wassernetzwerkes gemacht werden. Zur Analyse der Ergebnisse und zur Erstellung einer Kalibrierung zur Feuchtebestimmung, und weiterer Parameter, kommen chemometrische Methoden zur Anwendung, die speziell an die Bedingungen und die Messmethode angepasst und validiert werden. Im Rahmen dieser Arbeit wird neben bekannten Quereinflüssen zur Charakterisierung des Messsystems, wie z. B. der Abstandsabhängigkeit einer spektroskopischen Messung, der Einfluss einer Veränderung der Probentemperatur auf die Bestimmung der Materialfeuchte untersucht. Zur Auswertung der Spektren kommt ein multivariates Verfahren (PLS) zum Einsatz. Das Problem solcher multivariaten Modelle ist die Art der Kalibrierung. Die Kalibriermodelle zeigen sehr leicht große Fehler in der Bestimmung des Analyten wie z. B. dem Wassergehalt, sobald Varianzen in einem zu analysierenden Spektrum auftreten, die nicht im Kalibrierdatensatz repräsentiert sind [20, 21]. Es ist zwar durchaus möglich diese Modelle gegen eine Vielzehl an Varianzen wie z. B. der Korngröße einer Probe [9, 22] oder dem Anbaugebiet eines Rohstoffs, die beide ihrerseits Einfluss auf das Analyseergebnis haben, unempfindlich zu gestalten. Es lässt sich auch sagen, dass ein Modell robust gegen diese Quereinflüsse gestaltet werden kann, wenn die störende Varianz im Kalibrierdatensatz mit berücksichtigt ist. Leider büßt das Modell durch die Erhöhung der Robustheit an Genauigkeit ein, so dass der Vorhersagefehler größer wird [23]. Der Quereinfluss einer veränderlichen Probentemperatur spielt in diesem Zusammnenhang eine nicht zu vernachläsigende Rolle. Dazu wird zunächst untersucht, welchen Einfluss die Probentemperatur auf die Modellbildung hat. Es wurden bereits verschiedene Ansätze zur Temperaturkorrektur untersucht. So lässt sich in bestimmtem Maß die Vorhersage eines Modells auf die neue Varianz der Temperatur anpassen, indem die Regressionsgleichung der Materialfeuchtewerte (Vorhersage gegen Referenzwert) so transformiert wird, dass die fehlerbehafteten Vorhersagewerte auf den eigentlichen Referenzwert zurückgerechnet werden können [24]. Das Problem dieses Ansatzes ist jedoch die Festlegung des Modells auf genau diese Temperatur. Besonders aber gehen dadurch sämtliche Vorteile eines multivariaten Modells wie die Robustheit gegen diverse Quereinflüsse und die Ausreißererkennung verloren. Ein weiterer Ansatz, der bereits getestet wurde, ist die implizite Berücksichtigung des Temperatureinflusses im Kalibrierdatensatz [23, 25]. Hier werden Spektren, die bei variierender Temperatur gemessen wurden, mit in den Kalibrierdatensatz aufgenommen. Anschließend wird ein neues Modell erstellt. Die implizite Berücksichtigung als neue Varianz geht zu Lasten der Genauigkeit der Vorhersage des Modells. Es wird zwar robuster gegen den Einfluss der Temperatur, führt jedoch nicht immer zu einem akzeptablen Ergebnis. Ein neuer Ansatz, der im Rahmen dieser Arbeit untersucht wird, ist der Transfer des gesamten Kalibrierdatensatzes so, als wären diese Spektren bei der gewünschten Zieltemperatur aufgenommen worden. Der Vorteil dieses Ansatzes ist, dass die Varianz der Temperatur in diesem Fall nicht implizit ins Modell mit einfließst, sondern sogar aus dem Modell herausgehalten wird, da der bisher vorhandene Datensatz so verändert wird, dass sich das neue Modell auf Basis dieser Daten wie ein Modell verhält, das für die Zieltemperatur erstellt wurde. 


\section{Kapitel 2}

\section{Grundlagen der Materialfeuchtebestimmung}

Wasser spielt eine zentrale Rolle bei der Herstellung von Nahrungsmitteln. Aber auch in vielen anderen Produktentstehungsprozessen, z. B. in der Pharmazie und der chemischen Industrie, ist die Verwendung von Wasser zwingend notwendig. So kann z. B. ein zu hoher Wassergehalt im abhärtenden Beton eines Neubaus zum Aufquellen und somit auch Aufbrechen eines neu verlegten Holzparketts führen. Der Anteil des Wassers während eines Produktionsprozesses kann von entscheidender Bedeutung für die Qualität des erzielten Endproduktes sein. So hat der Anteil des Wassers bei Nahrungsmitteln einen direkten Einfluss auf die Lagerfähigkeit und Qualität. Bei vielen Produkten würde ein zu hoher Wasseranteil zum Verklumpen der Produkte oder im Fall von Getreide sogar zur Selbstentzündung führen. Auch die Haltbarkeit aufgrund der Ansiedlung von Pilzsporen würde in diesem Fall merklich minimiert werden. Der Wasserbestandteil kann sogar sicherheitsrelvant sein. So würde ein zu niedriger Anteil des Wassers in der Kunststoffproduktion eine höhere Sprödigkeit des Kunststoffes bewirken. Dies könnte bei Verwendung des Kunststoffes in der Automobilindustrie als Stoßfänger sogar zu der Verminderung der Steifigkeit und somit zu einer direkteren Impulsfortpflanzung in die Fahrgastzelle führen. Beschrieben wird der Wassergehalt einer zu analysierenden Probe durch den Begriff der Materialfeuchte. Aber auch bereits hier muss die sprachliche Besonderheit der Materialfeuchte berücksichtigt werden (zur genaueren Erörterung siehe Kapitel 2.2). Die Materialfeuchte wird zum Einen wie beschrieben als Synonym für den Wassergehalt eines Materials verwendet. Hierbei handelt sich aber streng genommen um die wasserselektive Materialfeuchte. Zum Anderen wird eine weitere nichtselektive Definition der Materialfeuchte verwendet. Sie ist definiert als Summe aller bei Erwärmung flüchtigen Bestandteile einer Probe, wie z. B. Wasser, Lösemittel, Öle, Fette, usw. Eine gängige Definition der Materialfeuchte bezogen auf das Feuchtgewicht ist:

$$
\text { Materialfeuchte }=\frac{\text { Feuchtgewicht }- \text { Trockengewicht }}{\text { Feuchtgewicht }} \cdot 100 \% .
$$

Im Folgenden wird i.d. R. der Begriff Materialfeuchte als wasserselektive Materialfeuchte verwendet. Das hierbei betrachtete Wasser kann auf verschiedene Arten 
mit dem Material verbunden sein durch

- Adsorption

- Adhäsion

- Sorption

- chemische Bindung (Kristallwasser)

Eine genauere Erläuterung der Wasserbindungsarten ist in Kapitel $2.3 \mathrm{zu}$ finden.

\section{1 Übersicht der Techniken und Methoden zur Bestimmung der Materialfeuchte}

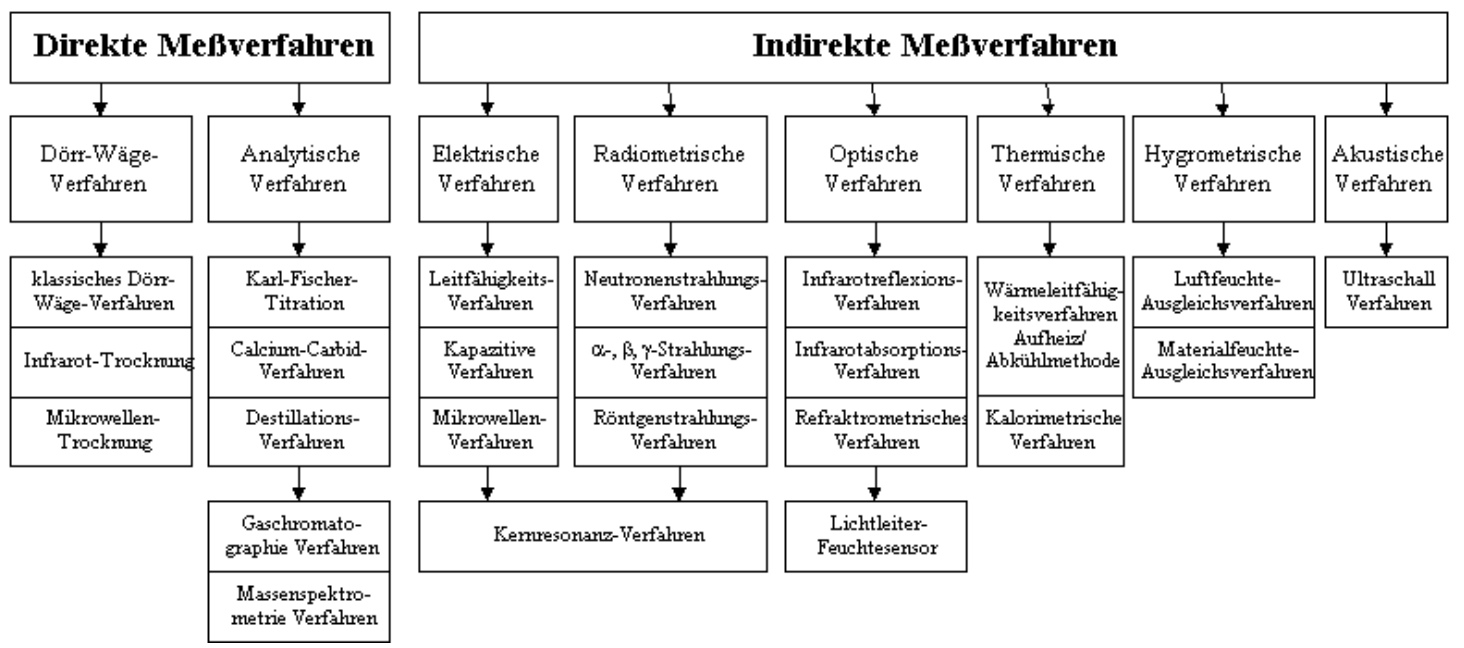

Abbildung 2.1: Übersicht der verschiedenen Feuchtemessverfahren [26]

Die Bestimmung des Wassergehaltes, also der wasserselektiven Materialfeuchte, kann in Gasen, Flüssigkeiten und Feststoffen von Interesse sein. Eine Übersicht der direkten und indirekten Messtechniken zur Bestimmung sowohl der Gesamt- als auch der wasserselektiven Materialfeuchte zeigt Abbildung 2.1. Gasfeuchtesensoren ermöglichen die Bestimmung des Wassergehalts in gasförmigen Stoffen. Hierzu werden prinzipiell zwei Methoden zum Einsatz gebracht. Zum Einen wird das thermodynamische Energiegleichgewicht des Wärme- und Wasserdampftransports zwischen Messmedium und Sensor ausgenutzt. Als Beispiel hierfür sei die Bestimmung des Taupunktes erwähnt [27]. Bei der Bestimmung des Taupunktes wird die Veränderung der Wasseraufnahmefähigkeit eines Gases durch Verringerung der Gastemperatur ausgenutzt. Aufgrund der Abkühlung des Gases und des damit sinkenden Wasseraufnahmevermögens wird der Sättigungsdampfdruck des Wasser-Gasgemisches 
erreicht, so dass Wasser an der kühlenden Fläche auskondensiert. Über die Temperatur, bei der der Sättigungsdampfdruck des Gemisches erreicht ist und somit Wasser auskondensiert, kann auf den Wassergehalt des untersuchten Gases geschlossen werden. Als weiteres Beispiel sei hier die Anwendung von Faserhygrometern erwähnt [27], bei denen die spezifische Änderung der Faserlänge in Abhängigkeit von der Gasfeuchte zur Bestimmung des Wassergehalts genutzt wird. So werden z.B. Menschen- oder Tierhaare als Messfasern verwendet. Die Kalibrierung dieser aufgrund ihrer mechanischen Bauweise komplett stromlos betreibbaren Messgeräte findet in Klimaschränken bei definierten Temperatur- und Gasfeuchteeinstellungen statt. Als zweite Eigenschaft zur Bestimmung der Feuchte von Gasen sei hier die Bestimmung der Energieabsorption durch das Wasser bei Durchstrahlung des zu untersuchenden Gases erwähnt. Hierbei wird das Wasser-Gasgemisch mit elektromagnetischen Wellen definierter Wellenlänge durchstrahlt und die Absorption der Energie durch Differenzmessung bestimmt [27].

Bei der Bestimmung des Wassergehalts in flüssigen Substanzen können prinzipiell die Methoden der Materialfeuchtebestimmung in Feststoffen verwendet werden, wie sie später in diesem Kapitel noch ausführlich erläutert werden. Dabei muss jedoch berücksichtigt werden, dass aufgrund der freieren Bindung des Wassers in Flüssigkeiten gegenüber der festeren Bindung in Feststoffen bereits durch Gravitation inhomogene Mischungsverhältnisse in einer flüssigen Probe auftreten können. Dabei sind Verfahren, die auf der Einlagerung der Wassermoleküle in den Sensor beruhen, sehr empfindlich bei Flüssigkeiten, die eine zu Wasser ähnliche Molekülgröße aufweisen. Bei konstanten Druck- und Temperaturbedingungen kann auch der Dampfdruck zur Bestimmung der maximalen Wasserkonzentration herangezogen werden [27].

\subsection{Unterschied Gesamtmaterialfeuchte und wasserselektive Materialfeuchte}

Wie bereits bei der Einführung des Begriffs der Materialfeuchte erläutert wurde, gibt es zwei verschiedene Arten von Feuchte in einem Feststoff. Die sprachliche Unterscheidung ist meist nicht vorhanden oder nur inkonsequent berücksichtigt. Selten wird auf eine konsistente Definition geachtet. Es kann jedoch sehr wichtig sein, die jeweils genaue Bedeutung der Feuchte zu kennen. Spricht ein Zigarrenhersteller beispielsweise von einer Feuchte von 70\%, so haben nicht seine Zigarren einen derart hohen Feuchtewert, der Tabak würde in diesem Fall nicht lagerfähig oder gar komsumierbar sein. Vielmehr meint der Zigarrenhersteller die umgebende Luft der Zigarren, woraus wiederum in Abhängigkeit vom herrschenden Luftdruck oder dem Wasserdampfpartialdruck eine Ausgleichsfeuchte in den Zigarren ensteht. Andererseits wird auch bei der Herstellung von organischen Membranen für den Einsatz in biotechnologischen Prozessen von einer Materialfeuchte gesprochen. Dies ist aber 
meist der gesamte Anteil aller bei Erwärmung flüchtigen Bestandteile. Es kann sogar so sein, dass die Feuchte in diesem Fall komplett wasserunabhängig ist, wenn lediglich organische Lösungsmittel in der Membran vorhanden sind. Generell werden in der chemischen Industrie alle flüchtigen Bestandteile einer Probe unter dem Begriff der Feuchte oder auch Materialfeuchte zusammengefasst. Es muss somit nicht zwangsläufig Wasser in der Probe enthalten sein. Bereits die beiden Begriffe Feuchte und Materialfeuchte werden i. d. R. als Synonym verwendet, so dass auch hier aufgepasst werden muss, dass der Begriff Feuchte in diesem Fall auch als Materialfeuchte verstanden und nicht mit der sonst üblichen Luftfeuchte verwechselt wird.

\subsection{Feuchte in festen und flüssigen Stoffen}

Der Begriff Feststofffeuchte bezieht den flüssigen, festen und gasförmigen Aggregatzustand des Wassers in Bindung mit Feststoffen ein. Zur Beschreibung von Wasser in festen Stoffen müssen bei der theoretischen Betrachtung die folgenden Größen mit einbezogen werden:

- Materialzusammensetzung

- Stoffdichte (Schüttdichte, Porosität)

- Temperatur

- Stoffkonzentration

- thermodynamische Größen

Der Zusammenhang zwischen Wasser, Wasserdampf und umgebender Matrix ist bei der Betrachtung von Wasser in einer Feststoffmatrix schwieriger als in der Gasphase, da spezifische Eigenschaften des jeweiligen Materials eine besondere Bedeutung haben. Das Wasser und der Wasserdampf stehen in einer Feststoff-

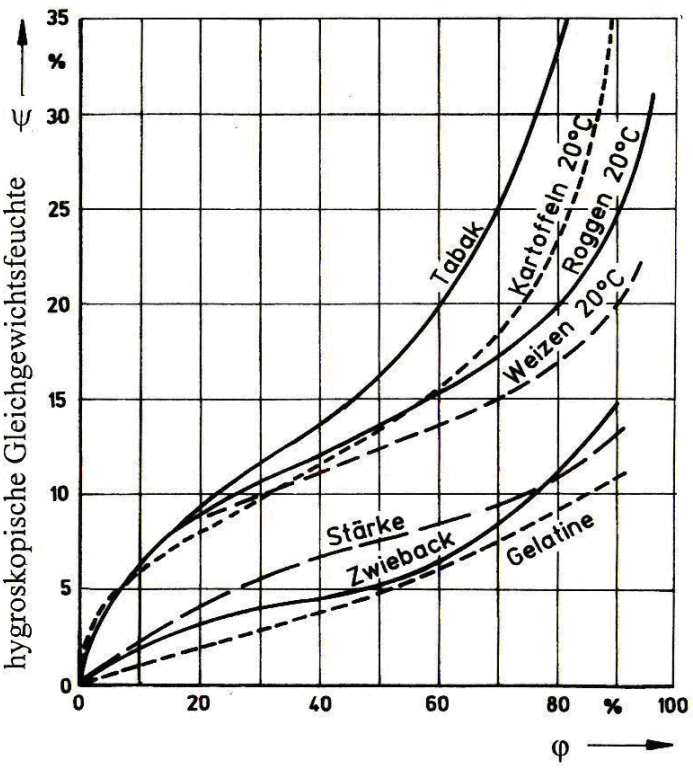

Abbildung 2.2: Sorptionsisothermen von Lebensmitteln [26], $\varphi$ : Luftfeuchte im Umfeld der Probe, $\Psi$ : Gleichgewichtsfeuchte che und den Kapillaren des Feststoffs. Bei einer Temperaturveränderung ändern sich folglich nicht nur die Gaszustände wie z. B. Dampfdruck, sondern auch die Materialeigenschaften wie z. B. Größe und Anzahl der Kapillaren. Die Veränderung der Materialkörnung kann eine starke Veränderung der hygroskopischen Eigenschaften des festen Stoffes nach sich ziehen. 
Der Grund hierfür ist die Änderung von Kapillar- und Porengrößen, da an diesen Stellen ein eigener von der Form und Größe des Volumens abhängiger Dampfdruck auftritt und somit die Bindungsenergie des Wassers davon abhängig ist. Meist muss zur theoretischen Betrachtung für jedes Material eine eigene Modellierung erstellt werden, da kein ideales Material zur Verfügung steht, von dem Gesetzmäßigkeiten abgeleitet werden können. Es lassen sich jedoch für jedes Material spezifische Adsorptionsisothermen bestimmen, die den Zustand der Wasseraufnahme und -abgabe bei konstantem Druck und konstanter Temperatur eindeutig charakterisieren. Aus der graphischen Darstellung der Adsorptionsisothermen lässt sich die Gleichgewichtsfeuchte des jeweiligen Materials in Abhängigkeit von der die Probe umgebenden Luftfeuchte für die Temperatur bestimmen, bei der die Adsorptionsisotherme aufgenommen wurde (siehe Abbildung 2.2). Wasser kann in einer Feststoffmatrix unterschiedlich gebunden sein (siehe Abbildung 2.3):

\section{Adsorbat- oder Adsorptionswasser}

Das Adsorptionswasser wird auch als Haftwasser bezeichnet. Es handelt sich dabei um eine ein- oder mehrmolekulare Schicht an einer Feststoffpartikeloberfläche, die auf die Ausbildung von Wasserstoffbrückenbindungen mit den polaren Molekülen oder den Atomen an der Oberfläche des adsorbierenden Feststoffs zurückzuführen sind. Aus diesem Grund kann die vollständige Entfernung dieser Bindung abhängig von der Stärke der Wasserstoff-Feststoff-Brückenbindung mit hohem Energieaufwand verbunden sein. Typische Wasserstoffbrückenbindungsenergien liegen im Bereich um $20 \mathrm{kJmol}^{-1}$ [28].

\section{Tropfwasser}

Tropfwasser ist eine besondere Form des Adsorptionswassers. Das Wasser ist in diesem Fall durch sehr schwache Wasserstoffbrückenbindungen oder Van-der-WaalsKräfte gebunden. Die Energie der Wasserstoffbrückenbindung ist in diesem Fall jedoch größer als die Bindungsenergie, die durch Van-der-Waals-Wechselwirkungen hervorgerufen wird (typische Wasserstoffbrückenbindungsenergien liegen im Bereich um $20 \mathrm{~kJ} \mathrm{~mol}^{-1}$, typische Energien der Van-der-Waals-Dipol-Dipol-Wechselwirkungen liegen im Bereich von $2 \mathrm{~kJ} \mathrm{~mol}^{-1}$ zwischen ruhenden polaren Molekülen und sogar nur im Bereich von 0,6 kJ mol ${ }^{-1}$ zwischen rotierenden polaren Molekülen) [29]. Dieser energiearme Verbund kann bereits durch die eigene Schwerkraft des sich bildenden Wassertropfens gelöst werden.

\section{Kapillarwasser}

Das Kapillarwasser wird aufgrund des Kapillareffektes bei polaren Molekülen in den Zwischenräumen der Feststoffstruktur gebunden. Daher wird es auch als Zwischenraum- oder freies Wasser bezeichnet. Der Kapillareffekt beruht auf den Anziehungskräften zwischen dipolaren Molekülen und der umgebenden Struktur. Es handelt sich dabei um Van-der-Waals-Dipol-Dipol-Wechselwirkungen, die gleichzei- 
tig zu den dominierenden Wasserstoffbrückenbindungen auftreten. Die Begründung für die Dominierung der Wasserstoffbrückenbindungen ist wiederum das Verhältnis der typischen Bindungsenergien wie sie bereits für "Tropfwasser" ausgeführt wurden.

Die Oberflächenmoleküle einer Flüssigkeit werden von der umgebenden Struktur (hier die kristalline oder amorphe Festkörperstruktur) stärker angezogen als von den Flüssigkeitsmolekülen, so dass die Flüssigkeit in diesen Strukturen festgehalten wird. Der Grund hierfür ist wiederum, dass typische Bindungsenergien von Wasserstoff-

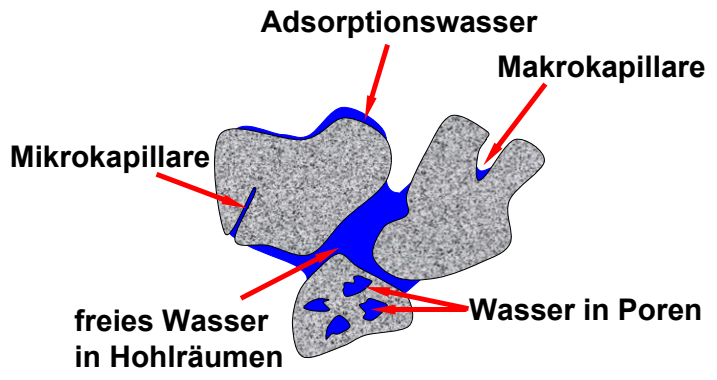
brücken zwischen Wassermolekülen und organischen Molekülen, die die Festkörperstruktur bilden, bis $\mathrm{zu} 40 \mathrm{~kJ} \mathrm{~mol}^{-1}$ betragen können [30] typische

Abbildung 2.3: Wasserbindungsarten Energien von Wasserstoffbrückenbindungen zwischen Wassermolekülen aber nur ca. $20 \mathrm{~kJ} \mathrm{~mol}^{-1}$ betragen [28]. Bei Kapillarwasser handelt es sich um eine typische für technische Prozesse interessante Bindungsart (Trocknung / Befeuchtung).

\section{Kristallwasser}

Kristallwasser ist chemisch in das Kristallgitter eines Feststoffes eingebunden. Die Bindung entsteht durch Ausbildung von Wasserstoffbrückenbindungen mit den Ionen der Kristalle. Bei Anionen werden die positiven Wasserstoffatome des Wassermoleküls von den negativen Ladungen angezogen, bei Kationen wird ein Wassermolekül durch die freien Elektronen des Sauerstoffs gebunden. Die hierbei auftretenden Anziehungskräfte sind relativ stark. Die gebundenen sogenannten Hydrationswassermoleküle bilden wiederum Wasserstoffbrückenbindungen zu weiteren Wassermolekülen, diese Wassermoleküle sind jedoch weniger stark gebunden als das direkte Hydrationswasser. Ein Beispiel für eine Verbindung, die Kristallwasser einlagert, ist Kupfervitriol oder auch Kupfer(II)-Sulfat, das bei eingelagertem Wasser eine blaue Farbe aufweist. Dargestellt wird der Kristallwasseranteil mit einem Punkt in der Summenformel. Für das Kupfervitriol gilt folgende Summenformel: $\mathrm{CuSO}_{4} \cdot 5 \mathrm{H}_{2} \mathrm{O}$. Demnach lagert es $5 \mathrm{H}_{2} \mathrm{O}$-Moleküle ein. Dabei binden 4 der Hydrationsmoleküle mit ihrem Sauerstoff an das Kupferatom und ein Hydrationsmolekül durch Wasserstoffbrückenbindung an das Sulfatanion [31]. 


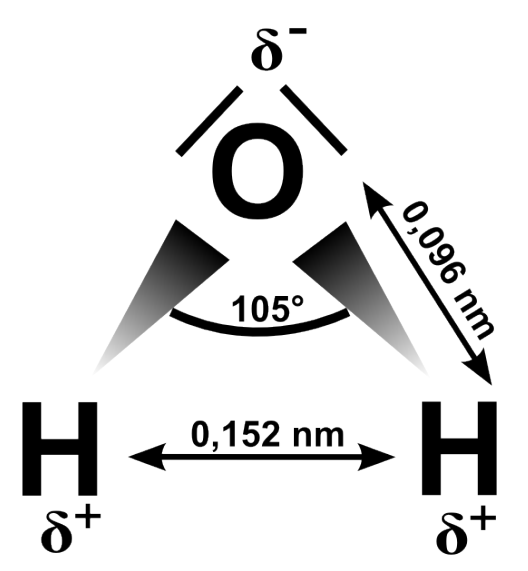

(a) geometrische Maße und Verteilung der Elektronegativitäten

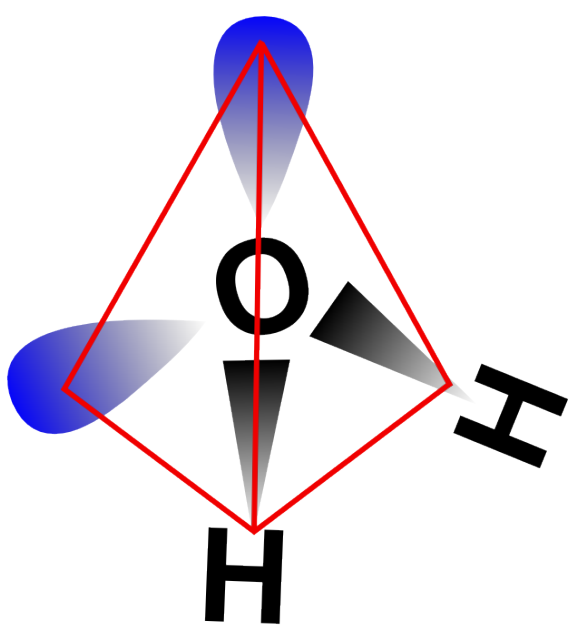

(b) Tetraederstruktur

Abbildung 2.4: Geometrie des Wassermoleküls

\subsection{Die chemische Verbindung Wasser}

Wasser ist eine der wichtigsten chemischen Verbindungen der Erde. Es stellt die Grundlage jeden Lebens auf der Erde dar. Seine Bedeutung ist so groß, dass es Wissenschaften gibt, die sich einzig mit der Verwendung, der Wirkung und Verteilung auf der Erde auseinandersetzen. Ohne die Verbindung aus Wasserstoff und Sauerstoff wäre unser Leben nicht möglich. So besteht der Mensch zu ca. $70 \%$ aus Wasser. Die meisten Pflanzenarten haben i. d. R. sogar einen Wasserbestandteil von mehr als $90 \%$.

Das Wassermolekül wird durch eine kovalente Bindung zwischen einem Sauerstoff- und zwei Wasserstoffatomen gebildet. Nach der Lewis-Valenz-Bindungstheorie [32] besteht eine kovalente Bindung aus einem gemeinsamen Elektronenpaar der miteinander gebundenen Atome. Die kovalente Bindung zwischen Wasserstoff und Sauerstoff ensteht durch die Überlappung der 1s-Orbitale der Wasserstoffatome und der $\mathrm{sp}^{3}$-Hybridorbitale des Sauerstoffs. Der Bindungswinkel der H-O-H Bindung müsste aufgrund der tetraedischen Struktur (siehe Abbildung 2.4), die durch die Anzahl der äußeren Elektronenpaare hervorgerufen wird, 109 betragen [28]. Durch die beiden nichtbindenden Elektronenpaare des Sauerstoffatoms werden die Bindungselektronenpaare jedoch abgestoßen, so dass die Sauerstoff-Wasserstoffbindungen lediglich einen Winkel von $105^{\circ}$ zueinander aufzeigen. Die unterschiedliche Elektronegativität von $\mathrm{H}^{+}$mit $\rho_{\mathrm{H}^{+}}=2,1$ und $\mathrm{O}^{2-}$ mit $\rho_{\mathrm{O}^{2-}}=3,44$ führt in dieser molekularen Verbindung zur Ausbildung von Dipolen (siehe Abbildung 2.4), da der Sauerstoff die Elektronen des Wasserstoffs stärker zu sich zieht, 


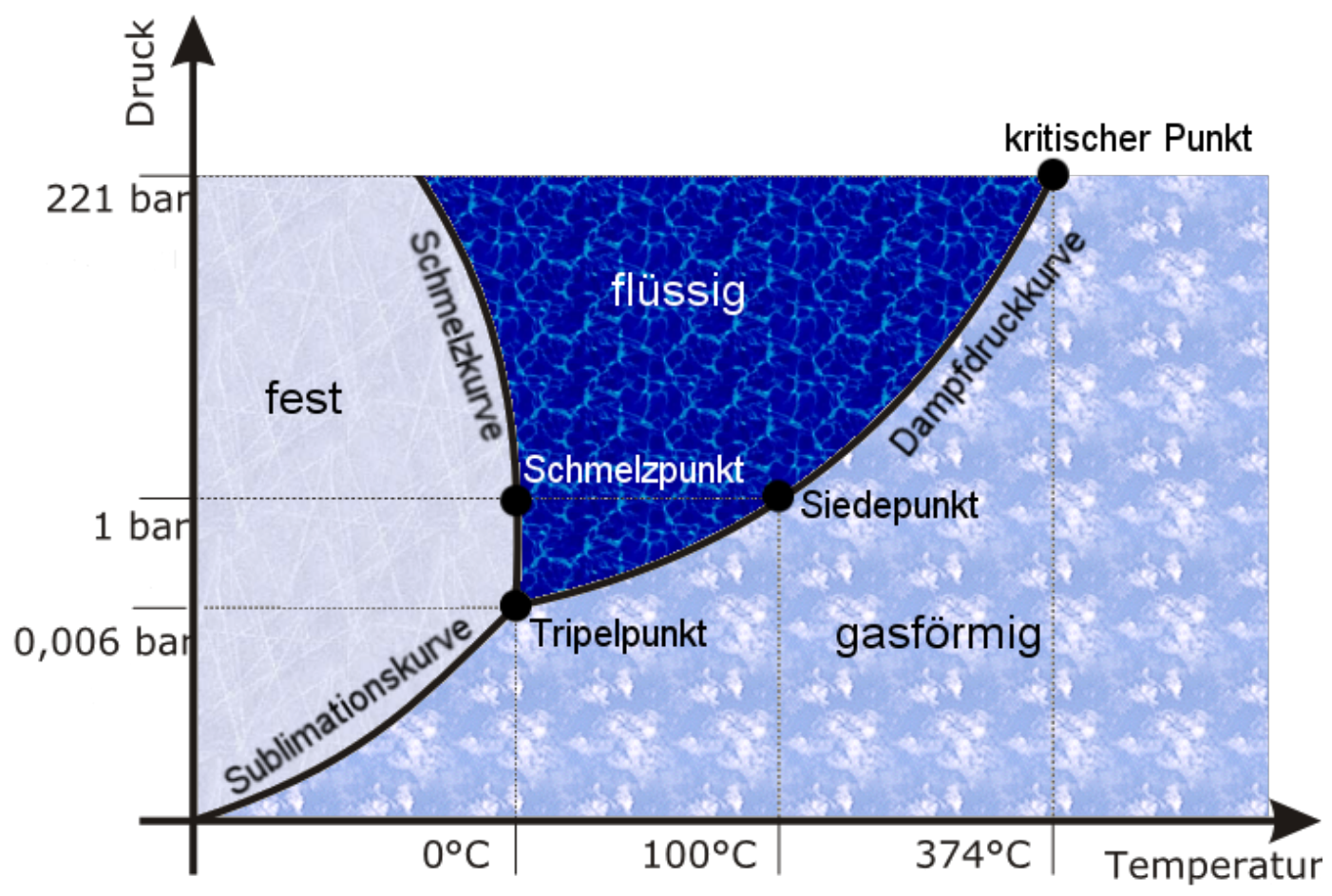

Abbildung 2.5: vereinfachtes Phasendiagramm von Wasser, Schmelzpunkt bei $0^{\circ} \mathrm{C}$, Tripelpunkt bei $0,01^{\circ} \mathrm{C}$

so dass sich in Richtung des Sauerstoffs eine negative Schwerpunktladung ausbildet. Die Energie der kovalenten Bindung von Wasserstoff und Sauerstoff beträgt ca. $463 \mathrm{~kJ} \mathrm{~mol}^{-1}$ [28]. Die Schwingungen des Wassermoleküls bewirken eine zusätzliche Verschiebung des Ladungsschwerpunktes, was zu einer weiteren Veränderung des Dipolmomentes führt. Der Dipolcharakter der Verbindung aus Sauerstoff und Wasserstoff führt zur Bildung von Wasserstoffbrückenbindungen mit typischen Bindungsenergien von $20 \mathrm{~kJ} \mathrm{~mol}^{-1}$ [29] zwischen den einzelnen Wassermolekülen [33], da es zu elektrostatischen Wechselwirkungen zwischen dem positiven Ladungsschwerpunkt um den Wasserstoff und dem negativen Ladungsschwerpunkt um den Sauerstoff kommt. Die tetraedische Struktur des Wassermoleküls ermöglicht bis zu vier Wasserstoffbrückenbindungen für ein einzelnes Molekül. Die damit einhergehenden Wassercluster (siehe Abbildung 2.6), also die festkörperartige Ausbildung einer Kristallstruktur in der flüssigen Phase (eine vereinfachte Darstellung der unterschiedlichen Wasserphasen zeigt Abbildung 2.5), werden unter Einwirkung von Temperaturänderungen in ihrer Struktur verändert. Die Umordnung dieser Strukturen ist ein Grund für die Anomalie des Wassers und führt z. B. bei der Erstarrung zur Volumenvergrößerung und einer damit einhergehenden Dichteabnahme. 
Die größte Dichte hat Wasser daher bei $\vartheta \approx 4^{\circ} \mathrm{C}$. Bei weiterer Temperaturerhöhung verringert sich die Dichte des Wassers analog zu anderen Flüssigkeiten. Der Siedepunkt von Wasser müsste aufgrund der molaren Masse des Wassermoleküls bei ca. $-80^{\circ} \mathrm{C}$ und der Schmelzpunkt bei ca. $-100^{\circ} \mathrm{C}$ liegen. Wird also die reine molare Masse betrachtet, so würde Wasser lediglich in einem Temperaturintervall von 20K im flüssigen Agregatzustand auftreten. In der Realität liegt der Schmelzpunkt jedoch bei $0^{\circ} \mathrm{C}$ und der Siedepunkt bei $100^{\circ} \mathrm{C}$. Der Grund der Abweichung der Sauerstoff-Wasserstoffverbindung von diesem Verhalten ist wiederum die Clusterbildung in der flüssigen Phase. Zum Brechen der dabei auftretenden Wasserstoffbrückenbindungen muss zusätzliche Energie aufgebracht werden, so dass sowohl der Schmelz- als auch der Siedepunkt höher liegen als es anhand der molaren Masse vorhergesagt würde. Nähere Erläuterungen zum Verhalten und den unterschiedlichen Arten von Wasserstoffbrückenbindungen folgen in Kapitel 2.5.4.
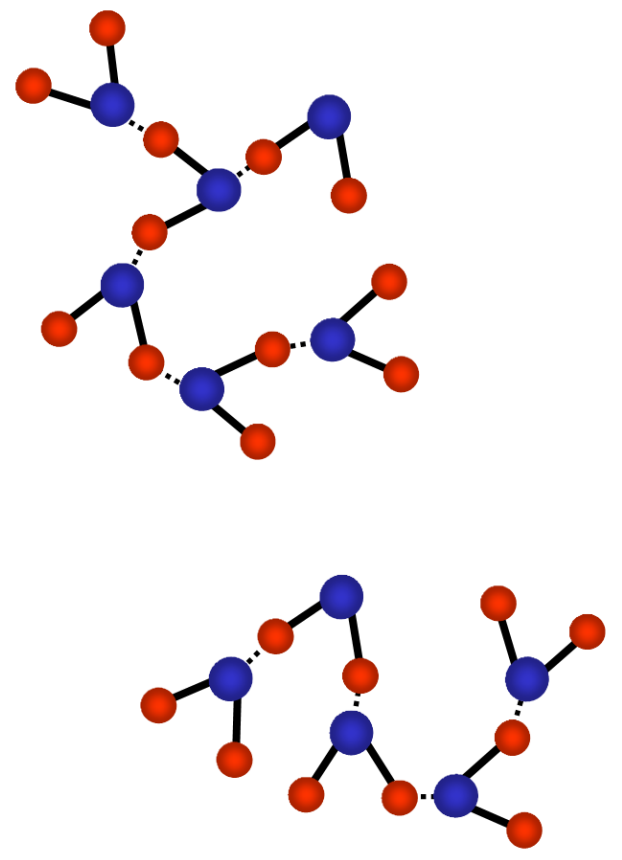

Abbildung 2.6: Bildung von Wasserclustern aufgrund der Wasserstoffbrückenbindungen (gestrichelte Linie) 


\subsection{Spektroskopische Eigenschaften des Wassermoleküls}

Zum Verständnis der spektroskopischen Eigenschaftem des Wassermoleküls müssen zunächst die mechanischen Schwingungseigenschaften des Moleküls betrachtet werden, die dann leicht auf das spektroskopische Verhalten übertragen werden können. Eine gutes Modell zur Beschreibung eines schwingenden Systems ist der harmonische Oszillator. Ihn $\mathrm{zu}$ verstehen bedeutet, auch das mechanische Verhalten von Molekularbindungen zu verstehen, da sich eine molekulare Bindung in ihren Grundzügen durch ein Masse-Feder-Masse-System beschreiben lässt. Die Schwingung des Wasseratoms lässt sich zunächst auf ein zweiatomiges Verhalten zurückführen, da die Schwingung der Wasserstoff-Sauerstoffbindung betrachtet wird.

\subsubsection{Der harmonische Oszillator}

Beim idealen harmonischen Oszillator wird die potentielle Energie $V$ des Oszillatorsystems lediglich durch einen quadratischen Term bestimmt:

$$
V=\frac{1}{2} \mathrm{k}\left(r-r_{e}\right)^{2}=\frac{1}{2} \mathrm{k} x^{2} .
$$

wobei k die Kraft-/ Federkonstante der Molekularbindung ist. $r$ ist die momentane Bindungslänge des Moleküls und $r_{e}$ die Bindungslänge in Ruhe. Die potentielle Energie weist eine parabolische Form auf, die symmetrisch zum Radius des Gleichgewichtszustands ist (siehe

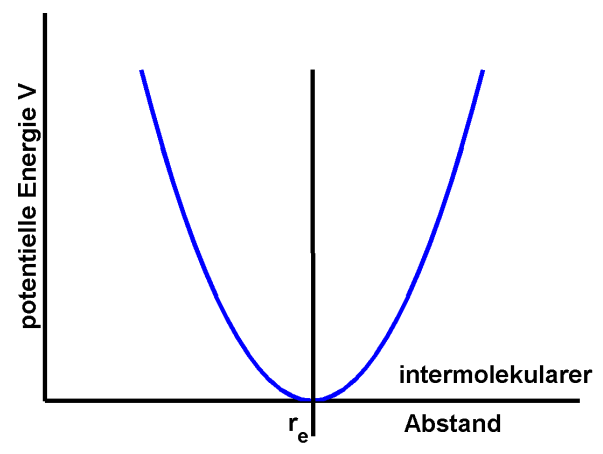

Abbildung 2.7: Potential des harmonischen Oszillators Abbildung 2.7). Die Lösung der Schrödinger-Gleichung [34] für den harmonischen Oszillator liefert als mögliche Eigenwerte für die Energieniveaus $E_{v}$ :

$$
E_{v}=\mathrm{h} \nu\left(v+\frac{1}{2}\right), \quad \text { mit } \quad v=1,2, \ldots
$$

wobei h das Planksche Wirkungsquantum und $v$ die Schwingungsquantenzahl ist, die Werte von $0,1,2, \ldots$ annehmen kann. Das quantenmechanische Schwingungsmodell für ein zweiatomiges Molekül führt zu der Schwingungsfrequenz $\nu$ :

$$
\nu=\frac{1}{2 \pi} \sqrt{\frac{\mathrm{k}}{\mu}},
$$


wobei $\mu$ die reduzierte molekulare Masse mit $\mu=m_{1} m_{2} /\left(m_{1}+m_{2}\right)$ ist und $m_{1}$ und $m_{2}$ die Massen der beiden gebundenen Atome sind. Üblicherweise werden statt der Frequenzen in der Spektroskopie Wellenzahlen angegeben, was mit dem Symbol $\tilde{\nu}$ von der Frequenzschreibweise $\nu$ unterschieden wird, da diese als Energieäquivalente betrachtet werden können:

$$
E_{v}=\operatorname{hc} \tilde{\nu}\left(v+\frac{1}{2}\right), \quad \text { mit } \quad \tilde{\nu}=\frac{\nu}{\mathrm{c}} .
$$

Die Gleichung 2.5 zeigt, dass sich die Schwingungsniveaus jeweils durch die gleiche Energiedifferenz $h c \tilde{\nu}$ unterscheiden. Für $v=0$ existiert zudem im quantenmechanischen System eine Nullpunktsenergie. Die Energieniveaus (siehe auch Abbildung 2.10 auf Seite 16) werden auch in der energieäquivalenten Wellenzahlschreibweise angegeben als $G(v)$ mit

$$
G(v)=\frac{E_{v}}{\mathrm{hc}}=\tilde{\nu}\left(v+\frac{1}{2}\right) .
$$

Die Auswahlregeln, welche Übergänge zwischen den Energieniveaus aktiv oder erlaubt sind, können vom Übergangsmoment $\boldsymbol{P}_{\boldsymbol{v}^{\prime \prime} \rightarrow \boldsymbol{v}^{\prime}}$ zwischen den einzelnen Energieniveaus abgeleitet werden:

$$
\boldsymbol{P}_{\boldsymbol{v}^{\prime \prime} \rightarrow \boldsymbol{v}^{\prime}}=\int \psi_{v^{\prime}}^{*} \varepsilon \psi_{v^{\prime \prime}} d \tau
$$

wobei $\psi_{v^{\prime}}$ und $\psi_{v^{\prime \prime}}$ die Wellenfunktionen der Zustände $v^{\prime}$ und $v^{\prime \prime}$ sind. $\varepsilon$ ist das Dipolmoment, welches für kleine Auslenkungen um den Ruhezustand, in dem das Dipolmoment den Wert $\varepsilon_{0}$ annimmt, als lineare Funktion von $x$ beschrieben werden kann:

$$
\varepsilon=\varepsilon_{0}+\left(\frac{d \varepsilon}{d x}\right) x
$$

Werden die jeweiligen Wellenfunktionen $\psi_{v^{\prime}}$ und $\psi_{v^{\prime \prime}}$ und das zugehörige Dipolmoment nach Gleichung 2.8 in die Gleichung 2.7 für das Übergangsmoment eingesetzt, ergeben sich die erlaubten Übergänge, wenn $\boldsymbol{P}_{\boldsymbol{v}^{\prime \prime} \rightarrow \boldsymbol{v}^{\prime}} \neq 0$ gilt. Dies wiederum zeigt, dass nur Moleküle, die eine Änderung des Dipolmomentes zeigen, schwingungsspektroskopisch aktiv sein können. Somit kommen hierfür nur Verbindungen voneinander unterschiedlicher Atome in Frage. Eine weitere Einschränkung des quantenmechanischen harmonischen Oszillators resultiert aus der Änderung der Schwingungsquantenzahl $v$ um jeweils nur eine Einheit. Folglich sind hier Übergänge zwischen nichtbenachbarten Energieniveaus verboten. Mathematisch müssen die erlaubten Übergänge die folgenden zwei Bedingungen erfüllen:

$$
\begin{gathered}
\left(\frac{d \varepsilon}{d x}\right) \neq 0, \\
\Delta v= \pm 1 .
\end{gathered}
$$


Bei Zimmertemparatur liegen die meisten Moleküle der Boltzmannverteilung [29] (siehe Gleichung 2.9 und Abbildung 2.8) folgend im Grundschwingungszustand mit $v=0$ vor, so dass der Fundamentalübergang $v=0 \rightarrow v=1$ das schwingungsspektroskopische Spektrum dominiert.

$$
N_{i}=N \frac{e^{-E_{i} / k T}}{\sum_{i} e^{-E_{i} / k T}}
$$

Die höheren Niveaus werden erst mit zunehmender Temperatur besetzt, so dass die höheren Schwingungsübergänge (die sogenannten „Hot Bands“, siehe Abbildung 2.10) wie $v=1 \rightarrow v=2$ etc. bei Zimmertemperatur fast nicht auftreten.

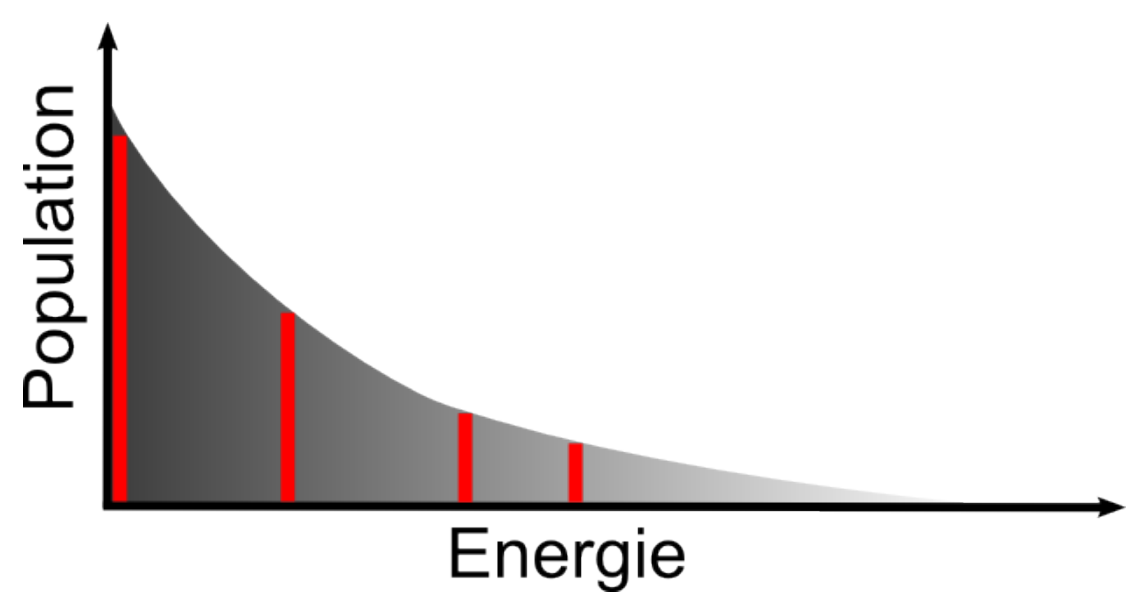

Abbildung 2.8: graphische Darstellung der Boltzmannverteilung 


\subsubsection{Der anharmonische Oszillator}

Bei der Betrachtung realer Moleküle lässt sich schnell feststellen, dass das Modell des harmonischen Oszillators das Verhalten der Moleküle nicht ausreichend wiedergibt. So lassen sich die nicht äquidistanten Energiedifferenzen zwischen höheren Energieniveaus im Vergleich zum Fundamentalübergang und auch die Dissoziation von Molekülen nicht erklären. Aber auch Obertonübergänge wie z. B. $v=0 \rightarrow v=2$ etc. sind erlaubt, so dass ein erweitertes Modell verwendet werden muss, um das Verhalten realer Moleküle besser beschreiben zu können. Die mechanische

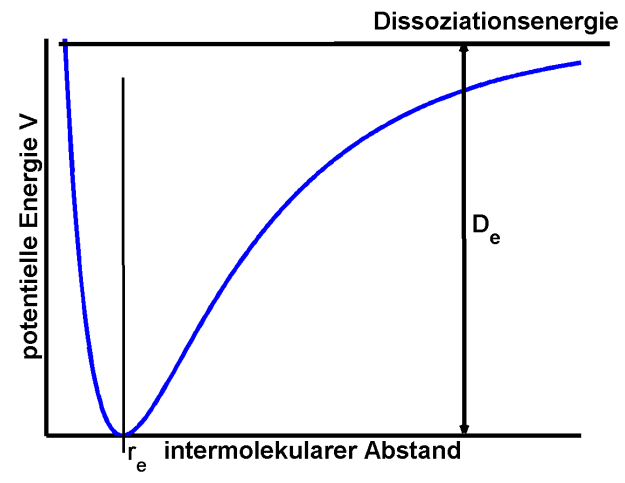

Abbildung 2.9: Potential des anharmonischen Oszillators, Morsepotential Anharmonizität ist einer der beiden Effekte, die zu der Abweichung vom harmonischen Oszillator hin zum erweiterten Modell des anharmonischen Oszillators führen. Erklärt werden kann diese Abweichung durch das Hinzunehmen kubischer und höherer Terme im Potential der mechanischen Schwingung:

$$
V=\frac{1}{2} \mathrm{k} x^{2}+\mathrm{k}^{\prime} x^{3}+\ldots, \quad \mathrm{k}^{\prime} \ll \mathrm{k} .
$$

Die Energieniveaus $G(v)$ (siehe Abbildung 2.10) können Nach Lösung der Schrödingergleichung für das Morse-Potential wie folgt beschrieben werden:

$$
\begin{aligned}
G(v)=\frac{E_{v}}{\mathrm{hc}} & =\tilde{\nu}\left(v+\frac{1}{2}\right)-x_{e} \tilde{\nu}\left(v+\frac{1}{2}\right)^{2} \\
& =\tilde{\nu}\left(v+\frac{1}{2}\right)-X\left(v+\frac{1}{2}\right)^{2} \\
& \operatorname{mit} X=x_{e} \tilde{\nu} .
\end{aligned}
$$

$x_{e}$ ist die Anharmonizitätskonstante. Durch den anharmonischen Term sind die Abstände der Energielevel nicht mehr äquidistant wie beim harmonischen Oszillator. Zur Beschreibung des anharmonischen Potentials (siehe Abbildung 2.9) wird die Morsefunktion verwendet:

$$
V=D_{e}\left(1-e^{-\beta x}\right)^{2},
$$

wobei $\beta$ konstant und $D_{e}$ die Dissoziationsenergie, gemessen vom Ruhezustand (Minimum der Morsefunktion), ist. Die Dissoziationsenergie wird in diesem Fall berechnet mit:

$$
D_{e}=\frac{\tilde{\nu}}{4 x_{e}} .
$$




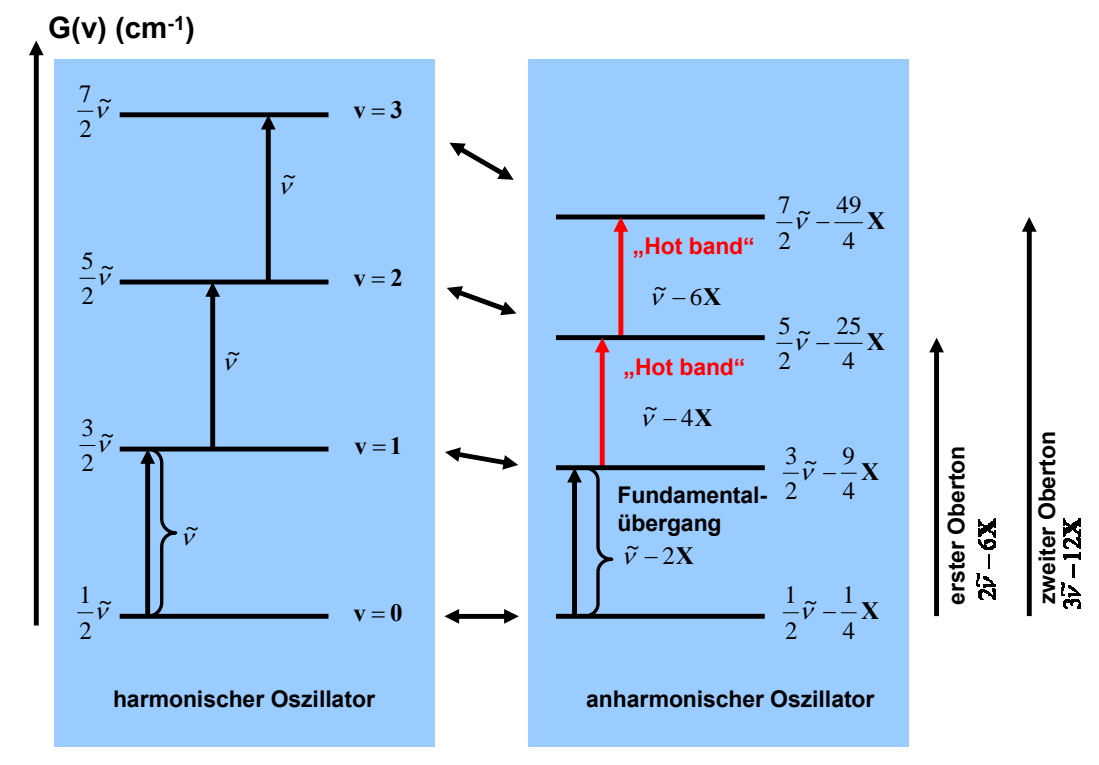

Abbildung 2.10: Energienieveaus des harmonischen und des anharmonischen Oszillators mit zugehörigen Übergängen

Die elektrische Anharmonizität, hervorgerufen von quadratischen und höheren Termen des Dipolmoments, ist der zweite Effekt, um den das Modell des harmonischen Oszillators erweitert werden muss. Dieser Effekt bewirkt Obertonübergänge zwischen Energieniveaus, die mehr als 2 oder 3 Schwingungsquantenzahlen auseinander liegen, so dass $\Delta v=+2,+3, \ldots$ werden kann, und wird beschrieben mit:

$$
\varepsilon=\varepsilon_{0}+\left(\frac{d \varepsilon}{d x}\right) x+\frac{1}{2}\left(\frac{d^{2} \varepsilon}{d x^{2}}\right) x^{2}+\ldots
$$

Die Abbildung 2.10 veranschaulicht, dass die Abstände der Energieniveaus beim anharmonischen Oszillator mit steigender Schwingungsquantenzahl $v$ aufgrund der anharmonischen Terme im Gegensatz zum harmonischen Oszillator keine Äquidistanz mehr aufzeigen und somit die höheren Übergänge („Hot Bands") in ihrer Energiedifferenz nicht mehr dem Fundamentalübergang entsprechen. Folglich zeigen auch die Obertonübergänge nicht, wie vom harmonischen Oszillator zu erwarten, das 2, 3, .. Vielfache des Fundamentalübergangs. 


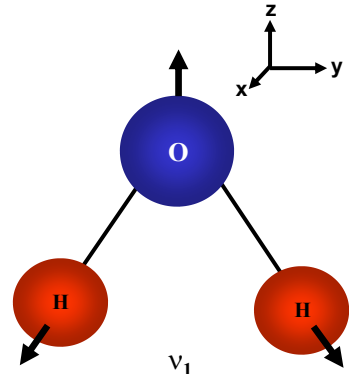

$3657 \mathrm{~cm}^{-1}$

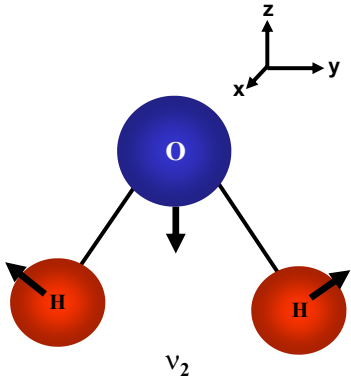

$1595 \mathrm{~cm}^{-1}$

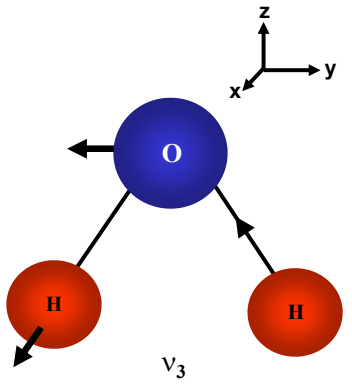

$3756 \mathrm{~cm}^{-1}$

Abbildung 2.11: Normal- / Grundschwingungen des Wassermoleküls, $\nu_{1}$ symmetrische Streckschwingung, $\nu_{2}$ Knickschwingung, $\nu_{3}$ asymmetrische Streckschwingung

\subsubsection{Normalschwingungen des Wassermoleküls}

Das Wassermolekül hat drei Freiheitsgrade bezüglich seiner Normal- oder Grundschwingungen. Dies lässt sich anhand der folgenden Betrachtung leicht nachvollziehen. Jedes Atom besitzt drei Freiheitsgrade zur Darstellung jeder möglichen Atomposition durch drei Koordinaten, z. B.x, y und z. Für ein N-atomiges Molekül gilt somit, dass es 3 N Freiheitsgerade im Raum besitzt. Davon beschreiben drei Freiheitsgrade die Translationsbewegung des Moleküls entlang der x-, y- und z-Achse. Drei weitere Freiheitsgrade beschreiben die Rotationsbewegungen des gesamten Moleküls um diese Achsen. Für ein nichtlineares Molekül verbleiben somit $3 N-6$ Freiheitsgrade, die die Bewegung der Molekülatomkerne relativ zueinander, also die Schwingungsbewegung, beschreiben [34].

Das dreiatomige Wassermolekül zeigt folglich drei Grundschwingungen (siehe Abbildung 2.11) bezüglich seiner Nulllage. Die erste Grundschwingung der OH-Bindung $\nu_{1}$ ist die symmetrische Streckschwingung und tritt bei einer Wellenzahl von $\tilde{\nu}_{1}=3657 \mathrm{~cm}^{-1}$ auf. Die Knick- oder auch Biegeschwingung $\nu_{2}$ zeigt die Absorption bei einer Wellenzahl von $\tilde{\nu}_{2}=1595 \mathrm{~cm}^{-1}$. Die asymmetrische Streckschwingung als dritte Grundschwingung des Wassermoleküls entspricht einer Wellenzahl von $\tilde{\nu}_{3}=3756 \mathrm{~cm}^{-1}$. Zur vereinfachten Veranschaulichung der Energieniveaus des Wassermoleküls wurde in Abbildung 2.12 das Energieschema für die zweiatomige $\mathrm{OH}-$ Bindung am Beispiel des harmonischen Oszillators dargestellt. Die einzelnen Energieniveaus werden durch Veränderung der jeweiligen Schwingungsquantenzahl $\left(v_{1}, v_{2}, v_{3}\right)$ erhalten, wie sie in der Abbildung den jeweiligen Energielinien zugeordnet 


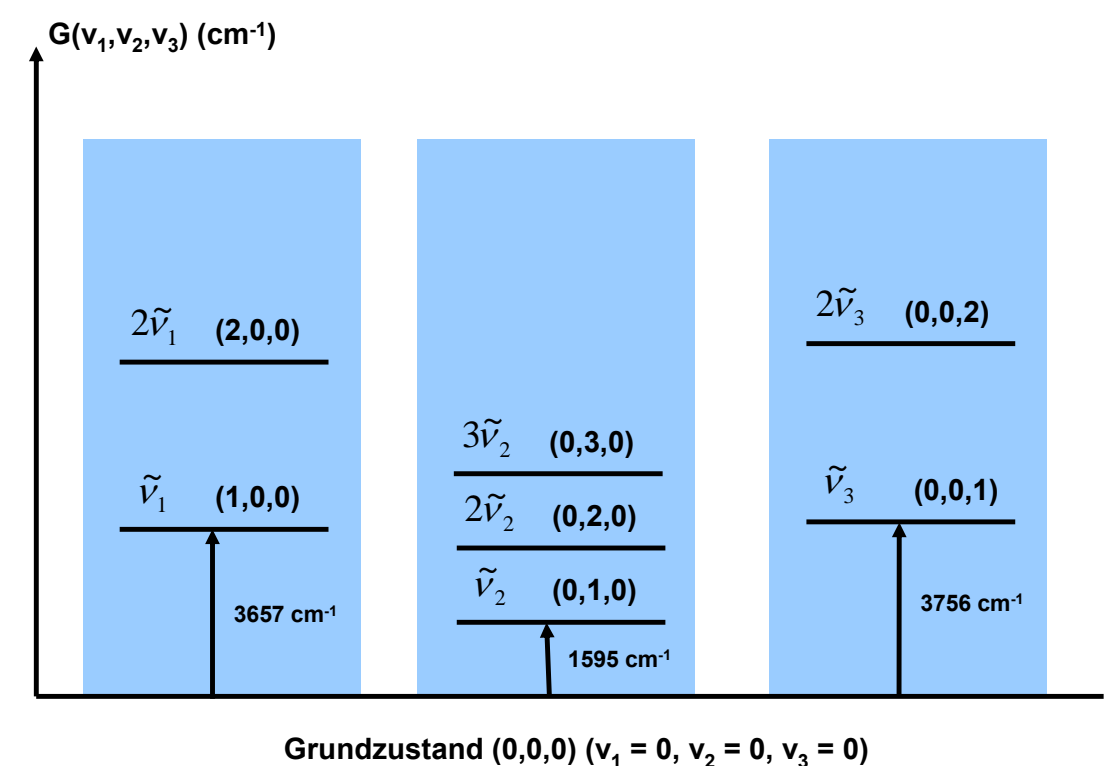

Abbildung 2.12: Schwingungsenergieniveaus des Wassermoleküls, vereinfachte Darstellung am Beispiel des harmonischen Oszillators für die $\mathrm{OH}-\mathrm{Bindung}$

sind. In diesem Fall werden die Energieniveaus beschrieben durch:

$$
\begin{aligned}
G\left(v_{1}, v_{2}, v_{3}\right) & =\frac{1}{\mathrm{hc}} E\left(v_{1}, v_{2}, v_{3}\right) \\
& =\tilde{\nu}_{1}\left(v_{1}+\frac{1}{2}\right)+\tilde{\nu}_{2}\left(v_{2}+\frac{1}{2}\right)+\tilde{\nu}_{3}\left(v_{3}+\frac{1}{2}\right),
\end{aligned}
$$

wobei $\tilde{\nu}_{1}, \tilde{\nu}_{2}, \tilde{\nu}_{3}$ jeweils die Schwingungswellenzahlen der drei Grundschwingungen sind. Der Grundzustand der Schwingungsenergie tritt für $v_{1}=0, v_{2}=0, v_{3}=0$ auf:

$$
G\left(v_{1}, v_{2}, v_{3}\right)=\frac{1}{2} \tilde{\nu}_{1}+\frac{1}{2} \tilde{\nu}_{2}+\frac{1}{2} \tilde{\nu}_{3}
$$

Die Energieänderung zwischen den Zuständen $G\left(v_{1}, v_{2}, v_{3}\right)$ und $G(0,0,0)$ berechnet sich mit

$$
\Delta G=\tilde{\nu}_{1} v_{1}+\tilde{\nu}_{2} v_{2}+\tilde{\nu}_{3} v_{3}
$$




\begin{tabular}{rrl}
\hline Wellenlänge $\lambda$ & Wellenzahl $\tilde{\nu}\left(\mathrm{cm}^{-1}\right)$ & Schwingungssymbol \\
\hline $6,08 \mu \mathrm{m}$ & 1645 & $v_{2}$, Knickschwingung \\
$3,05 \mu \mathrm{m}$ & 3277 & $v_{1}$, symmetrische Streckschwingung \\
$2,87 \mu \mathrm{m}$ & 3490 & $v_{3}$, asymmetrische Streckschwingung \\
$1900 \mathrm{~nm}$ & 5260 & $a v_{1}+v_{2}+b v_{3} ; a+b=1$ \\
$1470 \mathrm{~nm}$ & 6800 & $a v_{1}+b v_{3} ; a+b=2$ \\
$1200 \mathrm{~nm}$ & 8330 & $a v_{1}+v_{2}+b v_{3} ; a+b=2$ \\
$970 \mathrm{~nm}$ & 10310 & $a v_{1}+b v_{3} ; a+b=3$ \\
$836 \mathrm{~nm}$ & 11960 & $a v_{1}+v_{2}+b v_{3} ; a+b=3$ \\
$739 \mathrm{~nm}$ & 13530 & $a v_{1}+b v_{3} ; a+b=4$ \\
$660 \mathrm{~nm}$ & 15150 & $a v_{1}+v_{2}+b v_{3} ; a+b=4$ \\
$606 \mathrm{~nm}$ & 16500 & $a v_{1}+b v_{3} ; a+b=5$ \\
$514 \mathrm{~nm}$ & 19460 & $a v_{1}+b v_{3} ; a+b=6$ \\
\hline
\end{tabular}

Tabelle 2.1: Zuordnung einiger Absorptionsbanden von flüssigem Wasser

\subsubsection{Normalschwingungen des Wassermoleküls in der kondensierten Phase, flüssig oder in einer Feststoffmatrix}

Die bisherigen Grundlagen wurden alle an einem einzelnen Wassermolekül besprochen und können in erster Näherung zur Beschreibung des Verhaltens von Wasser in der Gasphase verwendet werden. Das schwingungsspektroskopische Verhalten von Wasser in der flüssigen Phase wird aber wiederum durch Wechselwirkungen zwischen den Wassermolekülen bestimmt. So verschiebt das Auftreten von Wasserstoffbrückenbindungen die Streckschwingungen $v_{1}$ und $v_{3}$ zu geringeren Wellenzahlen $\left(\tilde{\nu}_{1}=3277 \mathrm{~cm}^{-1}, \tilde{\nu}_{3}=3490 \mathrm{~cm}^{-1}\right)$ [35], da die Bildung der WasserstoffSauerstoff-Brücken zum „Aufweichen“ der intermolekularen OH-Bindungen durch Ladungsverschiebung führt. Das damit einhergehende Anwachsen des Dipolmomentes ist wiederum grund für das Anwachsen der Länge dieser Bindung. Das Anwachsen der Bindungslänge bewirkt letztendlich, dass die Streckschwingung niederfrequenter schwingt. Die Knick- oder auch Biegeschwingung $v_{2}$ wiederum wird aufgrund des Auftretens von Wasserstoffbrückenbindungen zu einer höheren Wellenzahl $\tilde{\nu}_{2}=1645 \mathrm{~cm}^{-1}$ verschoben [36]. Einige ausgewählte Absorptionsbanden (Schwingungs- und Kombinationsbanden) sind in Tabelle 2.1 zusammen mit den zugehörigen Schwingungssymbolen aufgeführt.

Die Stärke der Wasserstoffbrückenbindungen hat folglich einen direkten Einfluss auf das Schwingungsverhalten des Wassermoleküls. Somit bewirken Veränderungen in der Umgebung eines jeden Wassermoleküls signifikante Linienverbreiterungen, da die jeweilige lokale Stärke der Brückenbindung von der Art der umgebenden Brückenbindungen abhängt. Diese Art wird davon bestimmt, ob es sich bei der be- 


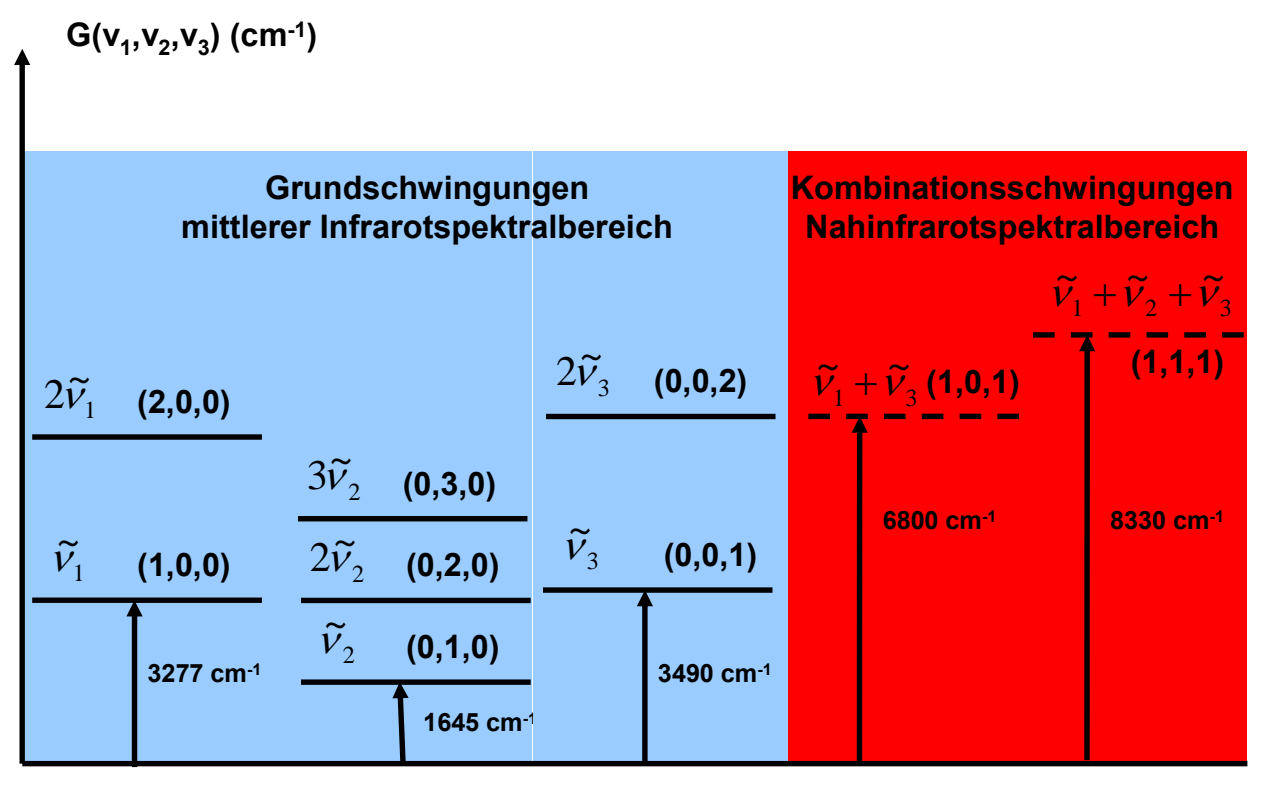

Grundzustand $(0,0,0)\left(v_{1}=0, v_{2}=0, v_{3}=0\right)$

Abbildung 2.13: Schwingungsenergieniveaus des Wassermoleküls in der flüssigen Phase

trachteten Brückenbindung um ein Brückenbindung gebendes oder akzeptierendes Molekül handelt, da dieses Verhalten eine unterschiedliche Stärke der Schwingungsverschiebung zu Folge hat. Dabei verändern sich die brückenbindungakzeptierenden Moleküle stärker in ihrer Schwingungsenergie als die bindungsgebenden Moleküle. Zum Verständnis dieses Vorgangs muss zunächst der Unterschied zwischen dem Geben und dem Akzeptieren einer Brückenbindung verstanden werden.

\section{Kooperative und antikooperative Wasserstoffbrückenbindungen}

Die Bildung einer Wasserstoffbrückenbindung bewirkt die Umverteilung der Elektronen sowohl in dem wasserstoffgebenden als auch in dem wasserstoffakzeptierenden Molekül, was wiederum die Möglichkeit weitere, neue Brückenbindungen zu bilden, beeinflusst. Im wasserstoffgebenden Molekül wird die Elektronendichte in der Ionenpaarregion, also der Region der freien Elektronen des Sauerstoffmoleküls, erhöht [37], da der Einfluss des nun gebundenen Wasserstoffatoms auf die Sauerstoffelektronen geringer wird. Dies wiederum erhöht die Akzeptanz weiterer Wasserstoffbrückenbindungen, da nun die Anziehungskraft der Elektronen um das Sauerstoffatom stärker geworden ist. Das wasserstoffakzeptierende Molekül wiederum verringert seine 
Elektronendichte um den intermolekularen Wasserstoff und gleichzeitig auch in der noch freien Ionenpaarregion, da die Elektronendichte in Richtung der Brückenbindung erhöht wurde. Dies hat wiederum zur Folge, dass dieses akzeptierende Molekül sein Bestreben, eine weitere gebende Wasserstoffbrückenbindung einzugehen, erhöht. Wiederum wird dadurch das Bestreben, eine akzeptierende Brückenbindung durch die Verringerung der Elektronendichte in der noch ungebundenen Ionenpaarregion zu bilden, verschlechtert. Die Elektronenumverteilung aufgrund der Bildung einer Wasserstoffbrückenbindung bewirkt sowohl das kooperative als auch das antikooperative Verhalten der Brückenbindungen. Dabei bedeutet die Kooperativität einer Wasserstoffbrückenbindung, dass die Akzeptanz einer Brückenbindung die Abgabe einer weiteren Brückenbindung bewirkt. Wiederum bedeutet die Inkooperativität einer Brückenbindung, dass die Akzeptanz einer Brückenbindung die Akzeptanz einer weiteren Brückenbindung verschlechtert. Eine detaillierte Beschreibung mit ausführlicher graphischer Darstellung möglicher Wasserstoffbrückenbindungen liefert die Arbeit von Chaplin [38].

Die hohe Diversität der möglichen Wasserstoffbrückenbindungsarten und der damit einhergehende Einfluss auf die Schwingungseigenschaften der Wassermoleküle im flüssigen Zustand bewirkt, dass die Schwingungsbanden gerade im Bereich der Kombinationsschwingungen (NIR-Spektralbereich, siehe auch Abbildung 2.13) nicht scharf begrenzt, sondern über einen Bereich im Spektrum ausgedehnt sind. Zusätzlich ist die Länge der Wasserstoffbrückenbindung nahezu linear temperatur- und druckabhängig [39].

\subsubsection{Oberton- und Kombinationsschwingungen}

Die Anharmonizität, wie sie bereits im Kapitel 2.5.2 zum anharmonischen Oszillator beschrieben wurde, bewirkt neben den Obertonübergängen, für die $\Delta v_{i}>1$ ist, auch Kombinationsbanden. Diese Kombinationsbanden enstehen durch die Überlagerung verschiedener Grundschwingungsniveaus, bei denen folglich mehrere $\Delta v_{i} \neq 0$ auftreten. Diese Übergänge sind jedoch schwächer als die Fundamentalübergänge, da die Anharmonizitätsterme i. d. R. klein sind.

Oberton- und Kombinationsschwingungen, die $\Delta v_{i}=2$ oder $\sum \Delta v_{i}=2$ zeigen, sind Schwingungen, bei denen sich die Schwingungsquantenzahl $v_{i}$ für eine Schwingung um 2 Quantenzahlen oder bei zwei Schwingungen um jeweils eine Quantenzahl ändern. Solche Kombinationen werden binäre oder zweifache Kombinationen genannt. Entsprechend werden Schwingungsänderungen mit $\Delta v_{i}=3$ oder $\sum \Delta v_{i}=3$ ternäre oder dreifache Kombinationen genannt. Die Abbildung 2.13 zeigt neben den Grundschwingungen, die sich im mittleren Infrarotspektralbereich in ihrer Absorption wiederfinden, auch Kombinationsschwingungen der drei Grundschwingungen $\tilde{\nu_{1}}, \tilde{\nu_{2}}, \tilde{\nu_{3}}$. Dabei handelt es sich um die Kombinationsschwingungen $\tilde{\nu_{1}}+\tilde{\nu_{3}}$ und $\tilde{\nu_{1}}+\tilde{\nu_{2}}+\tilde{\nu_{3}}$. Ob nun eine Schwingung infrarotaktiv ist oder nicht, also ob sich aufgrund der Schwingung das Dipolmoment ändert oder nicht, lässt sich beim Wassermolekül für die Grundschwingungen noch leicht erkennen. 


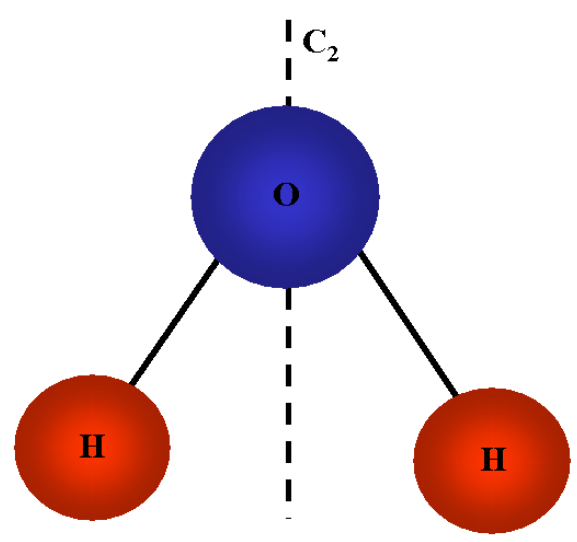

Abbildung 2.14: $\mathrm{C}_{2}$-Dreh- / Symmetrieachse des Wassermoleküls
Eine solche Beurteilung wird allerdings schwieriger, wenn es zu Ober- und Kombinationsschwingungen kommt. Um sicher sagen zu können, welcher Art eine solche Schwingung ist, müssen Symmetriebetrachtungen mit berücksichtigt werden. Die Symmetriebetrachtungen des Wassermoleküls zeigen, dass Wasser aufgrund seines Aufbaus eine Dreh- oder Symmetrieachse in vertikaler Richtung besitzt. Diese Achse wird nach Schönflies mit dem Symbol $\mathrm{C}_{2}{ }^{1}$ (siehe Abbildung 2.14) beschrieben [34]. Demnach führt eine Drehung um $\frac{2 \pi}{2}=\pi$ um die $\mathrm{C}_{2}$-Achse zu einer für einen stationären Beobachter nicht von der Ausgangskonfiguration zu unterscheidenden Anordnung des Wassermoleküls. Das Symmetrieelement $\mathrm{C}_{\mathrm{n}}$ ist gleichzeitig auch das Symbol für die zugehörige Symmetrieoperation, also der Drehung des Moleküls um $\frac{2 \pi}{n}$ um diese Achse.

Das aus Gleichung 2.7 auf Seite 13 bekannte Übergangsmoment $\boldsymbol{P}_{\boldsymbol{v}}$ für einen Schwingungsübergang bei einem zweiatomigen Molekül gilt ebenso für mehratomige Moleküle:

$$
\boldsymbol{P}_{\boldsymbol{v}}=\int \psi_{v^{\prime}}^{*} \varepsilon \psi_{v^{\prime \prime}} d \tau 4
$$

Folglich gilt:

$$
\begin{array}{lr}
\boldsymbol{P}_{\boldsymbol{v}}=0 & \text { für einen verbotenen Übergang und } \\
\boldsymbol{P}_{\boldsymbol{v}} \neq 0 & \text { für einen erlaubten Übergang. }
\end{array}
$$

Sind die beiden Schwingungszustände nichtentartet, so muss der Integrand aus Gleichung 2.18 totalsymmetrisch sein. Dies lässt sich in der totalsymmetrischen Darstellung wie folgt schreiben:

$$
\Gamma\left(\psi_{v^{\prime}}\right) \times \Gamma(\varepsilon) \times \Gamma\left(\psi_{v^{\prime \prime}}\right)=A
$$

wobei A der totalsymmetrischen Darstellung einer beliebigen, nicht-entarteten Punktgruppe entspricht. $\Gamma$ ist das Symbol für ,irreduzible Darstellung von“, auch zu verstehen als Symmetrierasse (näheres siehe [34]). Wird das Übergangsmoment in seine

\footnotetext{
${ }^{1}$ Schönflies-Nomenklatur: $\mathrm{C}_{\mathrm{n}}$, für Molekül mit n-facher Drehachse, Drehwinkel: $\frac{2 \pi}{n}$ im Bogenmaß, $n=2$ für Wassermolekül
} 
vektoriellen Komponenten in $x-, y$ - und $z$-Richtung zerlegt, so folgt die Schreibweise

$$
\begin{aligned}
P_{v, x} & =\int \psi_{v^{\prime}}^{*} \varepsilon_{x} \psi_{v^{\prime \prime}} d \tau \\
P_{v, y} & =\int \psi_{v^{\prime}}^{*} \varepsilon_{y} \psi_{v^{\prime \prime}} d \tau \\
P_{v, z} & =\int \psi_{v^{\prime}}^{*} \varepsilon_{z} \psi_{v^{\prime \prime}} d \tau .
\end{aligned}
$$

Für den Betrag des Übergangsmomentvektors gilt:

$$
\left|\boldsymbol{P}_{\boldsymbol{v}}\right|^{2}=\left(P_{v, x}\right)^{2}+\left(P_{v, y}\right)^{2}+\left(P_{v, z}\right)^{2} .
$$

Somit ist der Übergang $v^{\prime} \rightarrow v^{\prime \prime}$ erlaubt, wenn eine der Komponenten von Null verschieden ist. Das Dipolmoment ist ebenso vektorieller Natur und zeigt somit in eine bestimmte Richtung. Daher zeigt es die Symmetrieeigenschaften einer Translationsbewegung in gleicher Richtung. Allgemein lässt sich die Darstellung also wie folgt schreiben:

$$
\begin{aligned}
& \Gamma\left(\mu_{x}\right)=\Gamma\left(T_{x}\right) \\
& \Gamma\left(\mu_{y}\right)=\Gamma\left(T_{y}\right) \\
& \Gamma\left(\mu_{z}\right)=\Gamma\left(T_{z}\right) .
\end{aligned}
$$

Für Gleichung 2.21 lässt sich mit dieser Erkenntnis schreiben:

$$
\begin{array}{r}
\Gamma\left(\psi_{v^{\prime}}\right) \times \Gamma\left(T_{x}\right) \times \Gamma\left(\psi_{v^{\prime \prime}}\right)=A \\
\text { und } / \text { oder } \\
\Gamma\left(\psi_{v^{\prime}}\right) \times \Gamma\left(T_{y}\right) \times \Gamma\left(\psi_{v^{\prime \prime}}\right)=A \\
\text { und } / \text { oder } \\
\Gamma\left(\psi_{v^{\prime}}\right) \times \Gamma\left(T_{z}\right) \times \Gamma\left(\psi_{v^{\prime \prime}}\right)=A
\end{array}
$$

wiederum unter der Berücksichtigung, dass nicht alle drei Komponenten des Übergangsmoments von Null verschieden sein müssen, damit ein Übergang erlaubt ist. Ein Schema zum Finden der Auswahlregeln für ein bestimmtes Molekül kann wie folgt lauten [34]:

1. Punktgruppe des Moleküls bestimmen

2. irreduzible Darstellungen der Translation der entsprechenden Punktgruppe nachschauen

3. ausgehend vom Zustand $v^{\prime \prime}=0$ muss für erlaubte Übergänge gelten: 


\begin{tabular}{l|rrrr|cc}
\hline$C_{2 v}$ & $\mathrm{I}$ & $C_{2}$ & $\sigma_{x z}$ & $\sigma_{y z}^{\prime}$ & Rotationen & Translationen \\
\hline $\mathrm{A}_{1}$ & 1 & 1 & 1 & 1 & & $\mathrm{~T}_{\mathrm{z}}$ \\
$\mathrm{A}_{2}$ & 1 & 1 & -1 & -1 & $\mathrm{R}_{\mathrm{z}}$ & \\
$\mathrm{B}_{1}$ & 1 & -1 & 1 & -1 & $\mathrm{R}_{\mathrm{y}}$ & $\mathrm{T}_{\mathrm{x}}$ \\
$\mathrm{B}_{2}$ & 1 & -1 & -1 & 1 & $\mathrm{R}_{\mathrm{x}}$ & $\mathrm{T}_{\mathrm{y}}$ \\
\hline
\end{tabular}

Tabelle 2.2: Charaktertafel der $C_{2 v}$-Punktgruppe, I ist die Identitätsoperation, die für eine $C_{2 v}$-Punktgruppe immer eins ist (näheres siehe [34])

$$
\Gamma\left(T_{z}\right) \leftarrow A, \quad \Gamma\left(T_{x}\right) \leftarrow A, \quad \Gamma\left(T_{y}\right) \leftarrow A,
$$

wobei diese Schreibweise den Übergang der jeweiligen irreduziblen Darstellung der Punktgruppe in den totalsymmetrischen Zustand A darstellt. Mit Hilfe der Charaktertafel in Tabelle 2.2, die alle Symmetrieoperationen aufführt, die zur $C_{2 v}$-Punktgruppe gehören, und der Gleichung 2.26 können die erlaubten Übergänge mit dem Zustand $v=0$ aufgeschrieben werden:

$$
A_{1} \leftarrow A, \quad B_{1} \leftarrow A, \quad B_{2} \leftarrow A .
$$

Diese Übergänge sind entlang der $z-, x-$ bzw. $y$-Achse polarisiert. Für die drei Grundschwingungen des Wassermoleküls sind die Punktgruppencharaktere bezüglich der $C_{2}$-Achse und der Spiegeloperation $\sigma_{v}(x z)+1$ und +1 für $v_{1},+1$ und +1 für $v_{2}$ und -1 und -1 für $v_{3}$. Daher sind die irreduziblen Darstellungen dieser Normalschwingungen:

$$
\Gamma\left(\psi_{v_{1}}\right)=A_{1}, \quad \Gamma\left(\psi_{v_{2}}\right)=A_{1}, \quad \Gamma\left(\psi_{v_{3}}\right)=B_{2} .
$$

Bei einer Kombinationsschwingung wird entsprechend das Produkt der Einzelschwingungen zweier Darstellungen bestimmt, indem jeweils die Charaktere jedes Symmetrieelements miteinander multipliziert werden. Die hierbei zu beachtenden Regeln sind wiederum in [34] zu finden. Werden nun z. B. gleichzeitig die Normalschwingungen $v_{1}$ und $v_{3}$ angeregt, so ergibt sich für die Kombinationsschwingung der beiden Grundschwingungen die Symmetrie:

$$
\Gamma\left(\psi_{v}\right)=\Gamma\left(\psi_{v_{1}}\right) \times \Gamma\left(\psi_{v_{3}}\right)=A_{1} \times B_{2}=B_{2} .
$$

Die Symmetriedarstellung $B_{2}$ ist laut Charaktertafel in Tabelle 2.2 mit einer Translation in $y$-Richtung verbunden, so dass auch hier eine Polarisation des Dipolmomentes entlang der $y$-Achse vorliegt. Dies wiederum bedeutet, dass die Kombinationsschwingung der Normalschwingungen $v_{1}$ und $v_{3}$ IR-aktiv ist und somit im Infrarotbereich absorbiert. 


\section{Kapitel 3}

\section{Experimentelle Techniken}

\subsection{Referenzanalytik}

Der Messwert eines direkten Messverfahrens entspricht der zu bestimmenden Größe. Er wird durch den direkten Vergleich mit einer Bezugsgröße gewonnen, z. B. lässt sich die Masse bestimmen, indem eine Balkenwaage auf einen Massestandard abgeglichen wird. Indirekte Messverfahren bestimmen die zu untersuchende Größe durch Messung einer über eine Beziehung verknüpften Größe und Rücktransformation mit Hilfe dieser Beziehung auf die gesuchte Größe. Die für die Rücktransformation notwendige Beziehung zwischen direkt bestimmter und zu bestimmender Größe muss zunächst ermittelt werden. Das Festlegen der Beziehung zwischen gemessener und zu bestimmender Größe heißt Kalibrieren [40].

Für die Bestimmung der Materialfeuchte mit Hilfe der NIR-Spektroskopie ist die Absorbanz der Probe im nahen Infrarot, im Speziellen die Absorbanz bei bestimmten Wellenlängen des IR-Bereiches, ein Maß für den Wassergehalt. Ebenso ist bei der Mikrowellenresonanzmethode die Verschiebung der Resonatorgüte ein Maß für den Wassergehalt. Hier wirft sich zunächst die Frage auf, ob die Bestimmung der gesamten Materialfeuchte mit Hilfe dieser beiden Methoden überhaupt sinnvoll zu realisieren ist, da diese indirekten Methoden selektiv nur den Wassergehalt einer Probe und nicht den Materialfeuchtegehalt bestimmen. In der Regel ist davon auszugehen, dass Proben aus einer Charge immer das gleiche Verhältnis von Wasser- und NichtWasserfeuchte besitzen. Wird das indirekte Verfahren nun mit Hilfe von Proben aus der zu untersuchenden Charge kalibriert, so sollte die Bestimmung der Feuchte auch über den reinen Wassergehalt unter der Berücksichtigung eines Umrechnungsfaktors „Wassergehalt in Feuchte" durchführbar sein. Dieser Umrechnungsfaktor befindet sich jedoch bereits in der Kalibriergleichung, da als Referenzwert bereits die Feuchte und nicht der Wassergehalt verwendet wurde. 


\subsubsection{Gesamtfeuchtebestimmung nach dem Prinzip der Thermogravimetrie}

Der Begriff Thermogravimetrie hat den Ursprung in der altgriechischen und lateinischen Sprache und setzt sich zusammen aus therme (griech.) $=$ Wärme, gravis (lat. $)=$ Gewicht und metrum (lat.) $=$ Methode. Die Thermogravimetrie umfasst alle Verfahren der thermischen Analyse, die eine Gewichtsveränderung bestimmen, die eine Probe aufgrund von Wärmezufuhr erfährt. Einige thermogravimetrische Verfahren sind aufgrund ihrer Methodik auch unter den Begriffen „Wäge-Trocknungsverfahren“ (engl. ,loss-on-drying LOD“)oder „Dörr-Wäge-Verfahren“ [16] bekannt. Die Wäge-Trocknungsverfahren zählen zu den direkten Messverfahren und treiben sowohl die Oberflächen- als auch die Kapillarfeuchte aus einer Probe. Dabei wird zunächst das Feuchtgewicht einer Probe bestimmt und die Probe anschließend so lange getrocknet, bis sie eine Gewichtskonstanz aufzeigt, d. h. dass sich ihr Gewicht trotz weiteren Trocknens nicht mehr ändert. Dieses Gewicht entspricht dem Trockengewicht. Die Materialfeuchte berechnet sich nach der Gleichung 2.1 auf Seite 3.

\section{Trockenschrankverfahren}

Eines der weltweit am häufigsten verwendeten und dabei auch das älteste WägeTrocknungsverfahren zur Bestimmung der Materialfeuchte ist das Trockenschrankverfahren [16]. Wie bei allen Wäge-Trocknungsverfahren wird hier sowohl eine Waage als auch eine Trocknungsapparatur benötigt. Die Auflösung der verwendeten Waage ist stets in Abhängigkeit von der gewünschten Genauigkeit auszuwählen, sollte jedoch i.d.R. mindestens $0,1 \mathrm{mg}$ sein, um ein hinreichend genaues Ergebnis zu erreichen. Die Trocknungsapparatur ist bei diesem Verfahren ein Trockenschrank, der vereinfacht nichts anderes als ein auf Labor- und Industriebedürfnisse abgestimmter Ofen ist. Bei einem Trockenschrank wird die Methode der Konvektionstrocknung zum Trocknen der Probe ausgenutzt. Bei der Konvektionstrocknung wird warme Luft (i. d. R. $\vartheta=105^{\circ} \mathrm{C}$ bei Lebensmittelproben) über die Oberfläche der Probe und um das Probengefäß herum geleitet, so dass zunächst die Feuchte an der Oberfläche verdampft. Durch das Verdampfen der Oberflächenfeuchte entsteht ein Feuchtegradient zwischen dem Volumen und der Oberfläche der Probe. Daher strömt aus dem Volumen der Probe ständig neue Feuchte an die Oberfläche, wo sie verdampft bis irgendwann der Gleichgewichtszustand eintritt. Im Gleichgewichtszustand entspricht die an die Umgebung abgegebene Feuchte der aus der Umgebung aufgenommen Feuchte und bewirkt eine Konstanz der Feuchte in der Probe (ideal: Feuchte 0 \% F). Dieser Gleichgewichtszustand ist eingetreten, wenn die Gewichtskonstanz erreicht ist. Ein Nachteil der Trockenschrankmessung ist die lange Messzeit, welche inklusive der Vor- und Nachbereitungszeit mindestens 2 Stunden beträgt. Die Trockenschrankmethode ist i.d. R. das Standardreferenzverfahren für Materialfeuchte, auf das alle anderen Verfahren zurückführbar sein müssen, und wird je nach Anwen- 


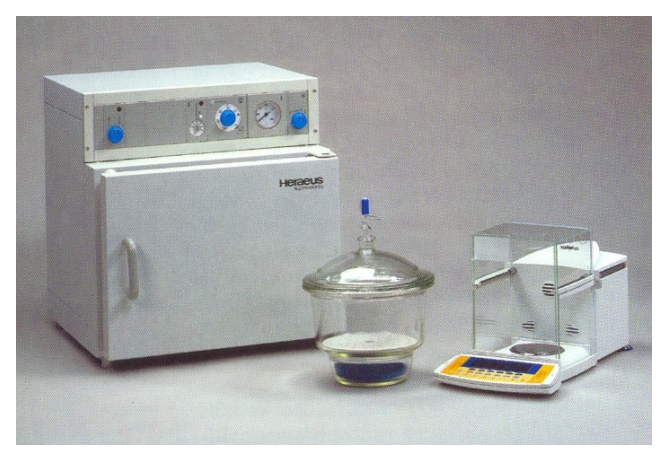

(a) Trockenschrank mit Waage und Exsikkator

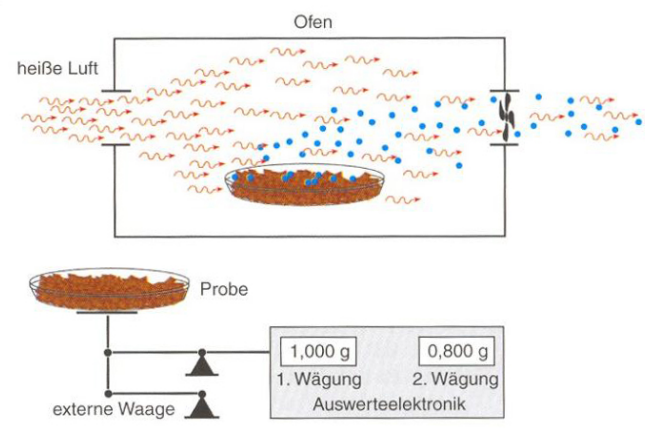

(b) Prinzip der Trockenschrankmethode

Abbildung 3.1: Trockenschrankmethode, Gerätedarstellung und Prinzipskizze [16]

dung in einer DIN-Norm festgelegt. Die DIN 52183 regelt z. B. das Vorgehen zur Holzmaterialfeuchtebestimmung mit der Trockenschrankmethode. Die Bestimmung der Materialfeuchte von Getreide nach der Trockenschrankmethode regelt die DIN 10350 [41].

\section{Materialfeuchteschnellbestimmer}

In vielen Bereichen der Industrie und der Wissenschaft kommt es auf die Schnelligkeit einer Methode oder eines Messgerätes an. So muss bei einer Prozesssteuerung häufig innerhalb weniger Minuten untersucht werden, wie hoch die Materialfeuchte eines Ausgangsstoffes oder eines Produktes ist. Hier zeigt die Trockenschrankmessung ihre Schwächen aufgrund der langen Messzeit. Vergleichbar gute Ergebnisse wie die Trockenschrankmethode geben Materialfeuchteschnellbestimmer bei einer deutlich verringerten Messdauer. Materialfeuchteschnellbestimmer sind Geräte, die nach dem thermogravimetrischen Prinzip die Materialfeuchte einer Probe bestimmen. Diese Geräte bestehen prinzipiell aus einer Waage und einer Heizeinheit, die sich über der Waagschale befindet. Genau wie bei der Trockenschrankmessung wird hier zunächst das Gewicht der feuchten Probe bestimmt und dann die Probe bis zur Gewichtskonstanz getrocknet. Anschließend wird das Gewicht im getrockneten Zustand bestimmt und nach Gleichung 2.1 auf Seite 3 die Materialfeuchte berechnet. Genauer betrachtet vereint ein Materialfeuchteschnellbestimmer die Funktion eines Trockenschrankes mit der Funktion einer Waage. Die Schnelligkeit der Messung im Vergleich zum Trockenschrank beruht auf der Art der Wärmeeinkopplung. Beim Trockenschrank wird die Wärme hauptsächlich konvektiv und somit sehr langsam in die Probe eingekoppelt, da die Wärme von der Oberfläche über Wärmeleitung in- 


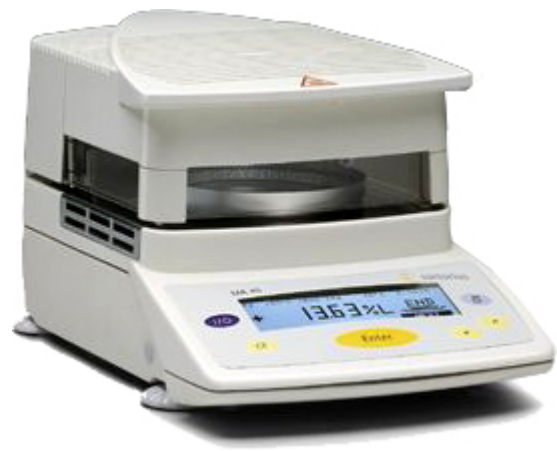

(a) MA45

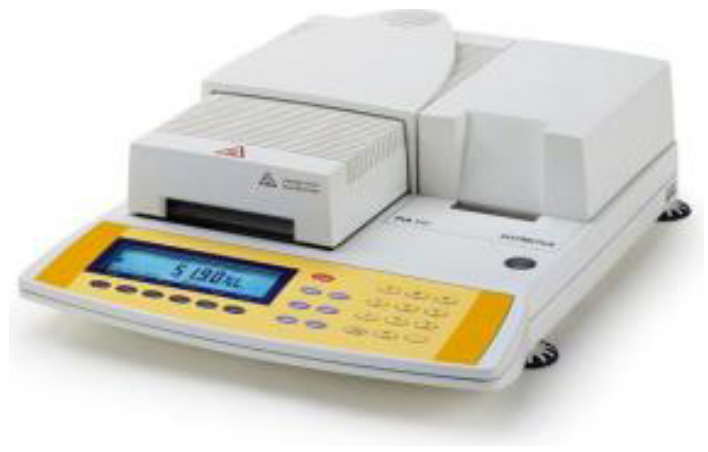

(b) MA100

Abbildung 3.2: Materialfeuchteschnellbestimmer MA45 und MA100 der Sartorius AG, MA ist Abkürzung für Moisture Analyzer

nerhalb der Probe erst verteilt werden muss. Beim Materialfeuchteschnellbestimmer hingegen wird die meiste Wärmeenergie über Wärmestrahlung eingekoppelt, die in die Probe eindringen kann. Erst dort wird die Wärmestrahlung in Wärmeenergie umgesetzt.

Die hohe Automatisierung der Messung eines Materialfeuchteschnellbestimmers vereinfacht den Messablauf, da der Nutzer im Routinebetrieb nur die Probe einbringen und die Messung starten muss. Aufgrund der ständigen Wägung der zu trocknenden Probe kann bereits während der Messung die momentane aus der Probe ausgetriebene Feuchte abgelesen werden. Zur Veranschaulichung von Materialfeuchteschnellbestimmern sind in Abbildung 3.2 exemplarisch zwei Geräte dargestellt. Die Materialfeuchteschnellbestimmer sind Rückführbar auf die Trockenschrankmethode, da die jeweilige Applikation für ein solches Gerät stets so entwickelt wird, dass die Ergebnisse dieser Referenzmethode mit den Materialfeuchteschnellbestimmern reproduziert werden kann. Der Begriff Applikation meint hier die Einstellungen der Parameter und die Vorschrift zur Handhabung der Probe. Diese Parameter sind u.a. Trocknungsart, Dauer und Temperatur der Trocknung. Diese Parameter werden auf jede Probe individuell angepasst. So wird Mehl bei einer anderen Temperatur als Kaffee getrocknet. Die Materialfeuchtewerte für die Mehlproben wurden mit der Applikationsvorschrift für den MA100 aus Tabelle 3.1 bestimmt. Die Ergebnisse der Mehlmessungen für Kapitel 5,6 und 7 sind in Tabelle 3.2 aufgelistet.

\subsubsection{Wassergehaltsbestimmung nach dem Prinzip der Coulometrie}

Die Coulometrie ist ein Absolutverfahren zur direkten Bestimmung von Stoffmengen. Hierbei wird die Konzentration eines elektrochemisch umgesetzten Stoffes aus der für diese Umsetzung benötigten Ladungsmenge (Produkt aus Stromstärke und 


\begin{tabular}{|c|c|}
\hline Einwaage & $5 \mathrm{~g}$ \\
\hline $\begin{array}{l}\text { Trocknungs- } \\
\text { temperatur }\end{array}$ & $110^{\circ} \mathrm{C}$ \\
\hline Trocknungsart & Standardtrocknung \\
\hline Trocknungsende & Vollautomatik \\
\hline Vorheizen & $\begin{array}{l}\text { Leermessung mit Aluscha- } \\
\text { le bei Trocknungstempera- } \\
\text { tur für } 10 \text { Minuten }\end{array}$ \\
\hline
\end{tabular}

Tabelle 3.1: Applikationsvorschrift des MA100 für Materialfeuchtebestimmung an Mehl

Zeit) mit Hilfe des Faradayschen Gesetzes [42] berechnet.

In dieser Arbeit wurde die Coulometrie u. a.zur Klärung der bereits in Kapitel 3.1 erwähnten Frage verwendet, inwieweit der thermogravimetrisch bestimmte Feuchtewert mit dem durch NIR-Spektroskopie indirekt gemessenen Wassergehalt korreliert. Wird der Wassergehalt selektiv bestimmt und gegen die Materialfeuchte aufgetragen, sollte sich ein funktioneller Zusammenhang zwischen Materialfeuchte und Wassergehalt ergeben, um eine Kalibrierung der Absorbanzmessung auf die Materialfeuchte vornehmen zu können.

Für die Untersuchungen im Rahmen dieser Arbeit wurde das Coulometer WDS 400

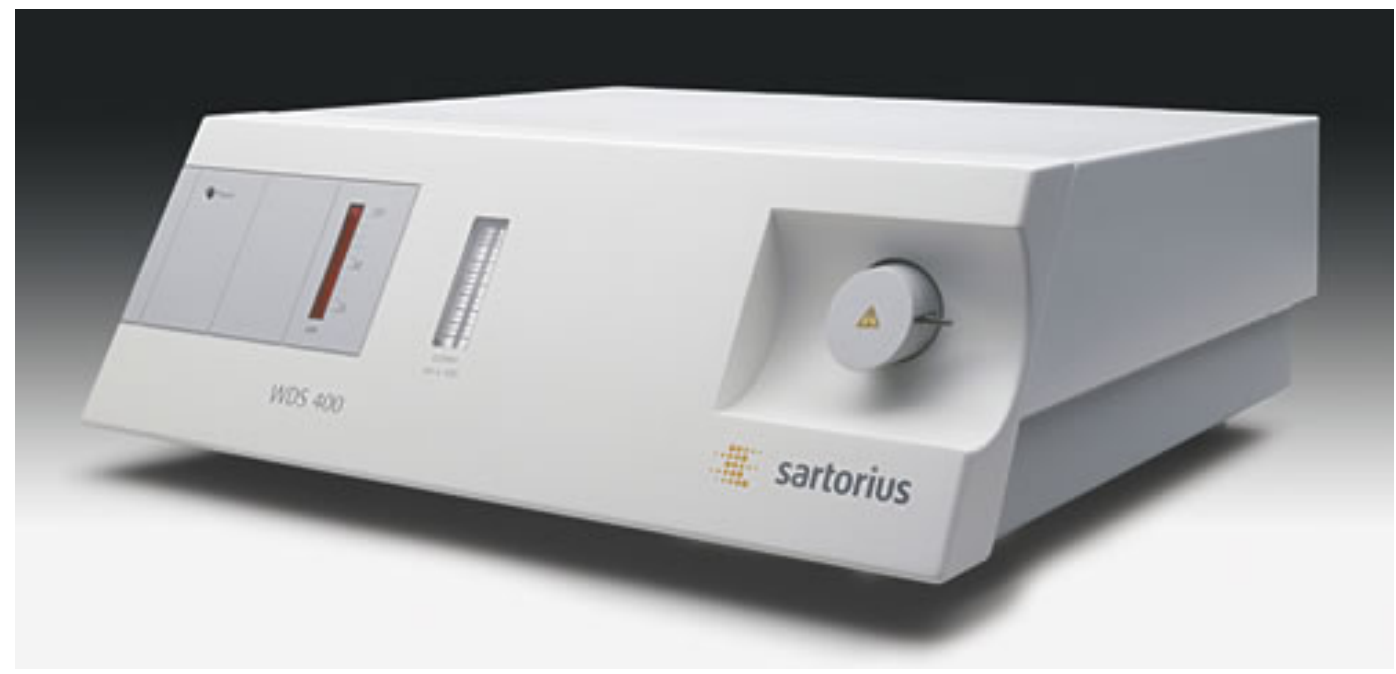

Abbildung 3.3: Darstellung des WDS 400

der Sartorius AG verwendet, das die selektive Bestimmung des Wassergehalts von

\footnotetext{
${ }^{1}$ WDS: water detection system
} 


\begin{tabular}{|llr|llr|}
\hline $\begin{array}{l}\text { Mehlbe- } \\
\text { zeichnung }\end{array}$ & $\begin{array}{l}\text { Befeuch- } \\
\text { tungsart }\end{array}$ & $\begin{array}{r}\text { Material- } \\
\text { feuchte in } \\
\text { \% F }\end{array}$ & $\begin{array}{llll}\text { Mehlbe- } \\
\text { zeichnung }\end{array}$ & $\begin{array}{l}\text { Befeuch- } \\
\text { tungsart }\end{array}$ & $\begin{array}{r}\text { Material- } \\
\text { feuchte in } \\
\text { \% F }\end{array}$ \\
\hline M09 & TRO & 8,23 & M12 & TRO & 6,29 \\
M09 & DIR & 10,57 & M12 & DIR & 10,45 \\
M09 & F35 & 10,14 & M12 & F35 & 8,79 \\
M09 & F76 & 12,37 & M12 & F55 & 12,37 \\
& & 14,68 & M12 & F76 & 13,29 \\
M13 & TRO & & M12 & H2O & 14,12 \\
M13 & DIR & 6,95 & M17 & TRO & 6,07 \\
M13 & F35 & 10,58 & M17 & DIR & 10,46 \\
M13 & F76 & 10,11 & M17 & F35 & 8,68 \\
M13 & H2O & 13,12 & M17 & F55 & 12,27 \\
& & 16,62 & M17 & F76 & 13,32 \\
M18 & TRO & & M17 & H2O & 14,02 \\
M18 & DIR & 6,27 & M19 & TRO & 5,77 \\
M18 & F35 & 10,48 & M19 & DIR & 10,22 \\
M18 & F55 & 8,83 & M19 & F35 & 9,91 \\
M18 & F76 & 12,26 & M19 & F55 & 11,68 \\
M18 & H2O & 13,59 & M19 & F76 & 13,45 \\
M21 & TRO & 15,02 & M19 & H2O & 16,43 \\
M21 & DIR & 6,59 & M45 & TRO & 5,48 \\
M21 & F35 & 10,74 & M45 & DIR & 10,78 \\
M21 & F76 & 10,35 & M45 & F76 & 13,93 \\
M21 & H2O & 12,73 & M45 & H2O & 18,16 \\
\hline
\end{tabular}

Tabelle 3.2: Materialfeuchtewerte der Mehlproben bestimmt mit MA100 nach Vorschrift aus Tabelle 3.1

festen und flüssigen Proben im Spurenbereich ermöglicht. Das WDS 400 vereint die Thermoanalyse und die Coulometrie in einem Gerät [43]. In Abhängigkeit vom vorgegebenen Temperaturverlauf kann zwischen den unterschiedlich stark gebundenen Wasseranteilen einer Probe (Oberflächenwasser, Kapillarwasser, Kristallwasser) unterschieden werden. Es besteht im Wesentlichen aus einem Quarzglasofen und einer Phosphorpentoxidzelle. Der Messablauf beim WDS beginnt zunächst mit dem Einwiegen der feuchten Probe auf einer separaten Mikrowaage in einem Messingschiffchen. Anschließend wird das Messingschiffchen mit der Probe in den Quarzglasofen des WDS gegeben. Dort wird die Probe nach einem vom Nutzer vorgegebenen Temperaturverlauf erwärmt. Der entstehende Wasserdampf wird von einem Gasstrom aus getrocknetem Stickstoff in die Phosphorpentoxid-Messzelle geleitet. Dort rea- 
giert das Phosphorpentoxid mit dem Wasser zu Phosphorsäure:

$$
\mathrm{P}_{2} \mathrm{O}_{5}+3 \mathrm{H}_{2} \mathrm{O} \longrightarrow 2 \mathrm{H}_{3} \mathrm{PO}_{4}
$$

Die Phosphorsäure wird anschließend elektrolytisch durch den Strom, der von der an der Messzelle anliegenden Spannung hervorgerufen wird, zu Phosphorpentoxid, Wasserstoff und Sauerstoff reduziert:

$$
4 \mathrm{H}_{3} \mathrm{PO}_{4}+W_{\text {elektrisch }} \longrightarrow 2 \mathrm{P}_{2} \mathrm{O}_{5}+6 \mathrm{H}_{2} \uparrow+3 \mathrm{O}_{2} \uparrow
$$

Aus dem Produkt der Stromstärke und der Zersetzungsdauer lässt sich die Ladungsmenge und daher über das Faradaysche Gesetz (siehe Gleichung 3.3) die abgeschiedene Masse berechnen. Die berechnete Masse entspricht der vom Phosphorpentoxid absorbierten und somit der aus der Probe ausgetriebenen Wassermenge.

Faradaysches Gesetz:

$$
m=\frac{M}{z F} \cdot Q=\frac{M}{z F} \cdot \int I \Delta t
$$

$\mathrm{m}$ ist die abgeschiedene Masse, $M$ die Molmasse, $Q$ die transportierte Ladung, $z$ die Ionenwertigkeit oder Ladungszahl [33] pro Molekül (hier: $z=3$, da $\mathrm{PO}_{4}^{3-}$ und $3 \mathrm{H}^{+}$), $I$ die Stromstärke, $F$ die Faraday-Konstante und $t$ die Zeit. Der Wassergehalt errechnet sich dann aus

$$
\text { Wassergehalt }=\frac{m_{\mathrm{H}_{2} 0}}{m_{\mathrm{feucht}}}
$$

und wird häufig in Prozent angegeben. 


\subsection{Spektroskopische Methoden}

Die Spektroskopie ${ }^{2}$ untersucht die Wechselwirkung elektromagnetischer Strahlung Lichtquelle mit Materie. Die Wechselwirkungen lassen sich in Absorption, Emission oder Streuung elektromagnetischer Strahlung an Atomen oder Molekülen einteilen. Bereits

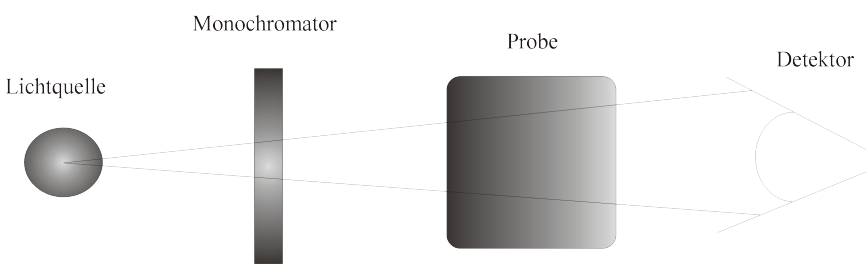

Abbildung 3.4: Transmissionsmessung

1665 zeigte Newton in seinen berühmten Experimenten die Zerlegung des weißen Lichtes in die Spektralfarben mit Hilfe eines dreieckigen Glasprismas [34]. Es gibt drei Hauptarten der Spektroskopie. Dies sind die Transmissions-, die Reflexions- und die Emissionsspektroskopie. Da die Emissionsspektroskopie in dieser Arbeit nicht verwendet wurde, wird auf eine nähere Erläuterung an dieser Stelle verzichtet (für nähere Informationen sei hier z. B. auf [44] verwiesen). Bei der Transmissionsspektroskopie werden die Eigenschaften wie z. B. die Absorbanz eines Stoffes bestimmt, indem die Probe von einer Lichtquelle bestrahlt wird und hinter der Probe das hindurch getretene (transmittierte) Licht detektiert wird (vgl. Abbildung 3.4). Bei der Reflexionsspektroskopie wird das von der beleuchteten Probe zurückgeworfene (reflektierte) Licht durch den Detektor aufgenommen (vgl. Abbildung 3.5). Das Ergebnis einer Spektroskopiemessung ist ein Spektrum. Ein Spektrum stellt i. d. R. die Intensität des transmittierten oder reflektierten Lichtes in Abhängigkeit von der Wellenlänge dar. In der (N)IR-Spektroskopie wird eine weitere Darstellung eines Spektrums verwendet. Hier wird die Intensität in Abhängigkeit von der Wellenzahl dargestellt. Die Wellenzahl berechnet sich aus der Wellenlänge mit:

$$
\tilde{\nu}=\frac{10000}{\lambda[\mu \mathrm{m}]} \cdot \frac{\mu \mathrm{m}}{\mathrm{cm}}
$$

Die mit Hilfe der Transmissions- und Reflexionsspektroskopie bestimmbare Absorbanz des eingestrahlten Lichts ist abhängig von der untersuchten Probe. So zeigen unterschiedliche Stoffe unterschiedliche Absorbanzen bei bestimmten Wellenlängen und somit besitzt jeder Stoff ein charakteristisches Absorbanzspektrum. Das Verhalten einer Probe lässt sich theoretisch für beide Spektroskopiearten berechnen. Die Absorbanz bei der Transmissionsmessung lässt sich mit Hilfe des Boguer-LambertBeer'schen Gesetzes bestimmen [44] und ist z. B. abhängig von der Konzentration und der Schichtdicke des Stoffes und der eingestrahlten Wellenlänge. Das Reflexionsverhalten einer Probe unter Voraussetzung einer diffusen (in alle Richtungen gleiche) Reflexion kann nach der Kubelka-Munk-Theorie approximiert werden [45].

\footnotetext{
${ }^{2}$ spectrum lat. $=$ Geist, Gespenst und skopein griech.$=$ schauen
} 


\subsubsection{NIR-Reflexionsspektroskopie}

Die Spektroskopie gliedert sich in verschiedene Bereiche abhängig von dem verwendeten Wellenlängenbereich. Eine dieser Spektroskopiearten ist die Infrarotspektroskopie, die ihre Wurzeln in einem Experiment von Sir William Herschel findet, das er um das Jahr 1800 durchführte.

Herschel untersuchte die Temperatur von Lichtstrahlen, indem er Sonnenlicht mit Hilfe eines Prismas aufteilte und die Teilstrahlen auf mehrere Thermometer lenkte. Die größte Temperaturzunahme erwartete er bei dem Thermometer, das mit dem intensivsten Licht, dem gelbgrünen, bestrahlt wurde. Das Thermometer, welches jedoch die höchste Temperaturzunahme anzeigte, wurde scheinbar von keinem Licht beleuchtet. Er bewies in weiteren Versuchen, dass es sich um eine für das menschliche Auge unsichtbare Strahlung handelt, die den Gesetzen der Optik folgt. Er nannte diese

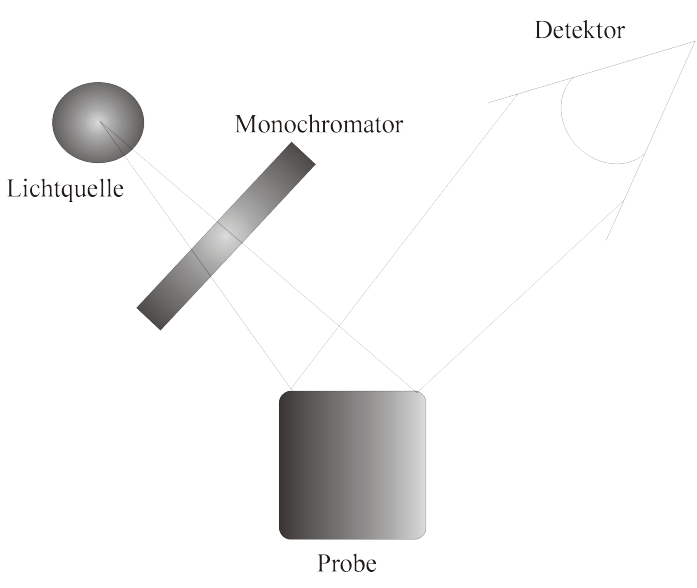

Abbildung 3.5: Reflektionsmessung Strahlung „Infrarot“ (jenseits von Rot), da die Strahlung bei seinem Versuch neben der Farbe Rot durch das entsprechende Thermometer angezeigt wurde [46]. Die NahInfrarot-Spektroskopie (NIR-Spektroskopie) gewinnt heute immer mehr an Bedeutung in Industrie und Forschung als ,nicht-invasive“ Analysetechnik [47]. So findet sie in der Forschung z. B. Anwendung bei der Bestimmung von Molekülparametern. Sie ist Werkzeug in den Agrar-, Tier-, Lebensmittel-, Pflanzen-, Polymer- und vielen weiteren Wissenschaften [48, 49]. In der pharmazeutischen Industrie wird sie z. B. zur In-Line-Überwachung der Medikamentenzusammensetzung eingesetzt [48]. Außerdem wird die NIR-Spektroskopie sehr häufig zur In-Line-Messung des Feuchtegehalts verwendet [50]. Wasser zeigt im NIR-Bereich, welcher sich in Wellenlängen ausgedrückt von $0,8 \mu \mathrm{m}$ bis $2,5 \mu \mathrm{m}$ erstreckt, ausgeprägte Absorbanzbanden (Spektralbereich, in dem ein Stoff Licht bestimmter Wellenlängen absorbiert). Es wurde bereits in vielen Publikationen [10, 18, 51, 52] gezeigt, dass zwei dieser Banden besonders gut zur Wassergehaltsbestimmung geeignet sind. Diese häufig verwendeten Banden liegen mit ihren Absorbanzmaxima bei $\lambda \approx 1,45 \mu \mathrm{m}$ und $\lambda \approx 1,94 \mu \mathrm{m}$. Die Abbildung 3.6(a) zeigt das Absorbanzspektrum von flüssigem Wasser. Die Abbildung 3.6(b) zeigt Absorbanzspektren von Mehl, an denen der Varianzbeitrag der veränderlichen Materialfeuchte an der Wasserbande bei $\lambda \approx 1,45 \mu \mathrm{m}$ zu erkennen ist. 


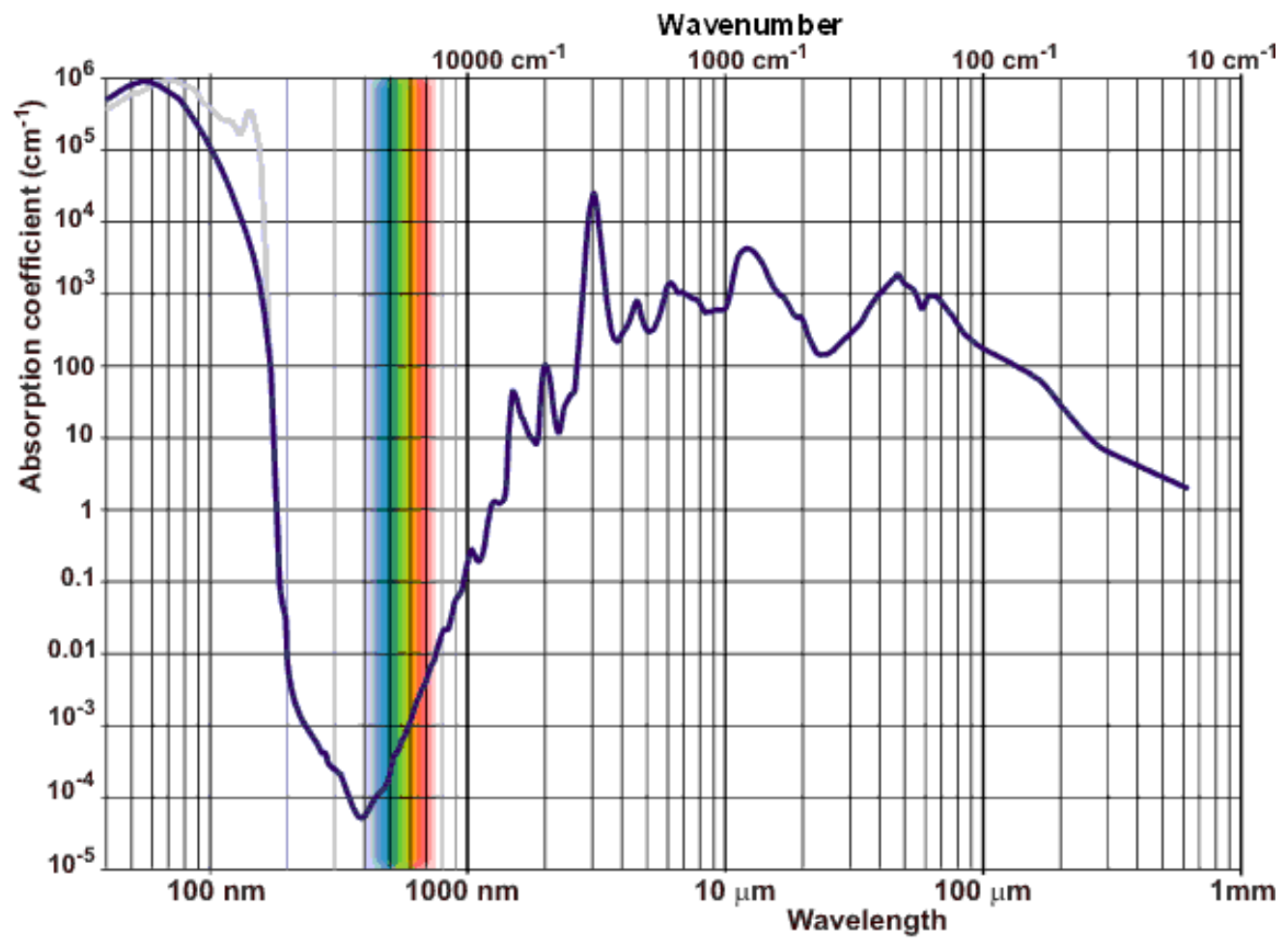

(a) Absorbanzspektrum von flüssigem Wasser [53]

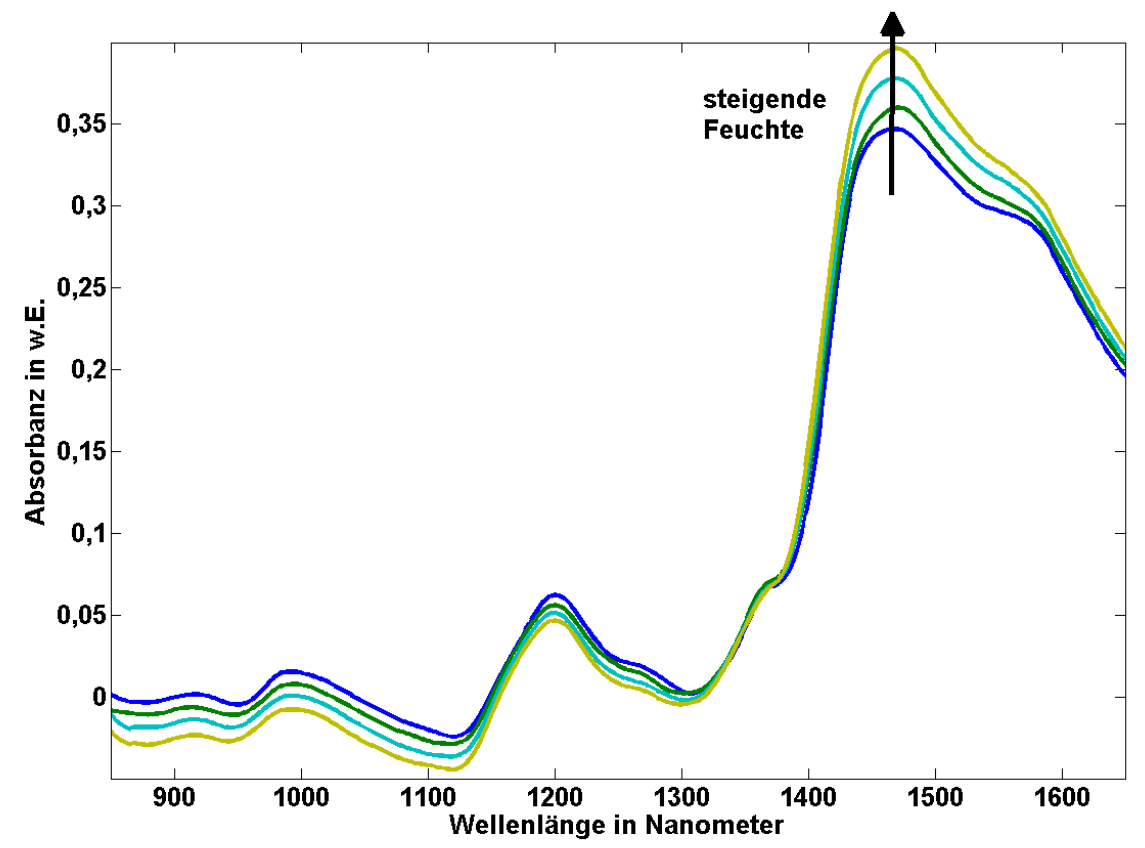

(b) Absorbanzspektrum von Mehl Type M12 (Bezeichnung siehe Kapitel A.1) zur Veranschaulichung des Varianzbeitrages durch Variation des Feuchtegehalts

Abbildung 3.6: Absorbanzspektren von Wasser und Mehl 


\subsection{Beschreibung des Versuchsaufbaus zur NIR- Spektroskopie}

In der NIR-Spektroskopie lassen sich die Varianzbeiträge durch veränderliche Materialfeuchte und den Quereinfluss einer variablen Probentemperatur nicht eindeutig unterscheiden, da beide Varianzen die Form der Wasserbande beeinflussen [54]. Zum besseren Verständnis wurden daher sowohl Untersuchungen an reinem Wasser als auch an Feststoffen mit unterschiedlichen Materialfeuchtegehalten durchgeführt. Die Aufnahme von Spektren an reinem Wasser ist aufgrund der hohen Absorbanz von Wasser jedoch nur in Transmission oder Transflektion möglich. Die Aufnahme von Spektren an Pulvern ist i.d. R. nur in diffuser Reflexion sinnvoll, so dass für beide Anwendungen ein eigener Versuchsaufbau verwendet wurde. Bei beiden Versuchen wurde ein Spektrometer vom Typ, wie er in Tabelle 3.13 auf Seite 48 beschrieben ist, verwendet.

\subsubsection{Aufbau zur Transmissionsmessung an Wasser}

Zur Aufnahme der temperaturabhängigen Spektren von reinem Wasser wurde eine temperierbare Küvette verwendet. Eine Skizze des Aufbaus und die Erläuterung der einzelnen Komponenten und Begriffe befinden sich in Abbildung 3.7. Die Küvette der Firma Hellma ${ }^{3}$ bietet durch ihre Konstruktion die Möglichkeit, temperiertes Wasser durch die äußere Wand zu leiten, so dass die zu untersuchende Flüssigkeit im Inneren des optischen Messvolumens auf konstante Temperatur gebracht werden kann. Die Temperatur der Küvette und somit der Messflüssigkeit wurde mit Hilfe eines Thermostaten (siehe Tabelle 3.11) und Wasser als Wärmemittel geregelt. Die Temperatur des zu untersuchenden Wassers im Innern des Küvettenvolumens wurde mit einem Thermoelement bestimmt. Hierzu wurde ein kleines Loch durch einen der PTFE-Stopfen gebohrt, die das Messvolumen abschließen. Um das Wasser im Messvolumen möglichst am Verdampfen zu hindern, wurde das Thermoelemt mit dem PTFE-Stopfen mit Hilfe des temperaturbeständigen Klebers Technosil (siehe Kapitel 3.3.2) verklebt. Ein Problem stellte die Lichtleitung des anregenden Lichtes in der Kühlwandung dar, da dieses zu einem zusätzlichen Signal auf dem Detektor führte. Um diesen Effekt zu minimieren, wurden auf beiden Seiten der Küvette sowohl Linsen (nähere Beschreibung weiter unten) in einer 1:1-Teleskopanordnung als auch Blenden angebracht, so dass sowohl kein Licht in die Außenwand der Küvette eingekoppelt werden konnte und auch kein Licht aus diesem Volumen wieder in die Detektionsfaser eingekoppelt werden konnte. Beim ersten Duchströmen der Küvette mit dem Temperierwasser muss darauf geachtet werden, dass keine Luftblasen im Temperiervolumen entstehen, um eine homogene Erwärmung der Küvette zu

\footnotetext{
${ }^{3}$ Bestellbezeichnung: Thermo-Küvette mit 2 PTFE-Stopfen 011.102, 165-QS, Schichtdicke $1 \mathrm{~mm}$, Volumen: $160 \mu \mathrm{l}$
} 


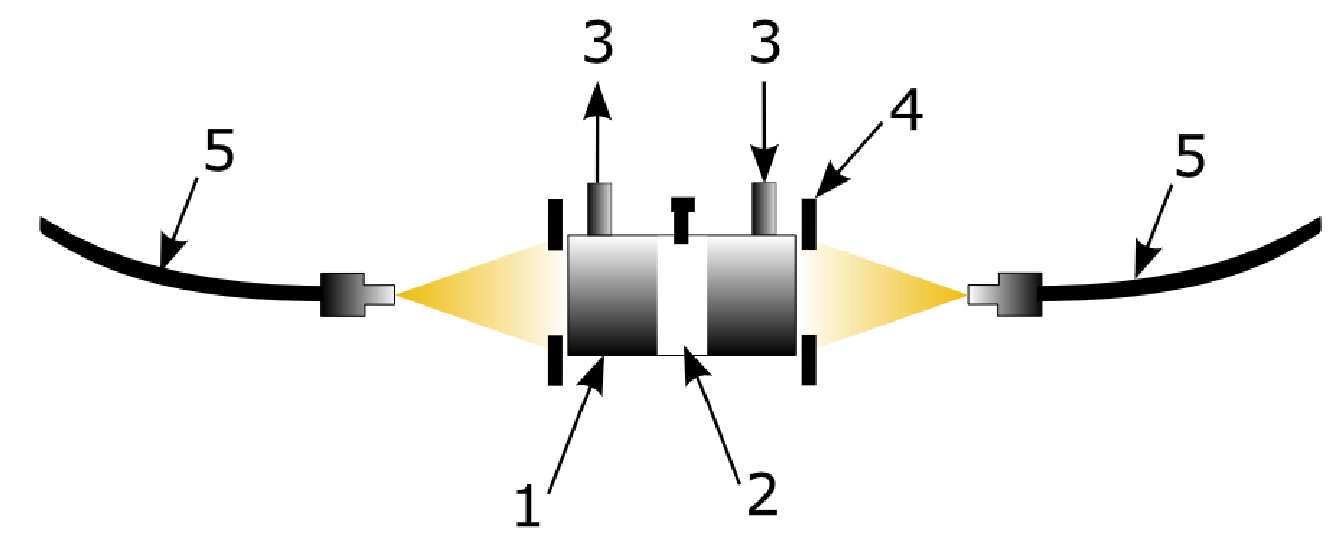

Abbildung 3.7: Schema des Aufbaus zu Transmissionsmessungen an reinem Wasser

1 temperierbare Küvette, $\mathbf{2}$ Messvolumen, $\mathbf{3}$ Temperierwasserzu- und ablauf, 4 Blende und abbildende Optik, 5 Lichtleitfaser mit SMA-Stecker

ermöglichen. Kleine Luftbläschen konnten hier durch leichtes Klopfen aus der Küvette getrieben werden. Zur besseren Thermostatisierung wurde der gesamte Aufbau zusätzlich komplett mit Schaumstoff umwickelt. Für die Bestimmung der Absorbanzspektren muss sowohl ein Leerspektrum (Messvolumen ohne Wasser) als auch ein Spektrum bei eingefülltem Wasser aufgenommen werden, um den Untergrund und die Veränderung des optischen Weges durch Temperaturänderungen herausrechnen aber auch die Absorbanz durch Quotientenbildung $I / I_{0}$ bestimmen zu können. Hierzu wurde die Temperatur der Küvette zunächst mit leerem Messvolumen auf die jeweils zu untersuchenden Temperaturschritte eingestellt. Im jeweils statischen Zustand wurde dann ein Transmissionsrohspektrum aufgenommen. Nachdem diese Reihe abgeschlossen war, wurde die Küvettentemperatur wieder auf die niedrigste Temperatur der Messreihe gebracht, indem der Thermostat entsprechend eingestellt wurde. Anschließend wurde deionisiertes Wasser in das Messvolumen eingebracht. In dieser Anordnung wurde wiederum die gleiche Temperaturreihe durchfahren und 
jeweils im temperaturstabilen Zustand das jeweilige Transmissionsrohspektrum für Wasser aufgenommen. Die Wasserspektren wurden in einem Temperaturbereich zwi-

\begin{tabular}{|c|c|c|c|c|c|c|c|}
\hline $\begin{array}{r}\text { am Thermostat eingestellte } \\
\text { Temperatur in }{ }^{\circ} \mathrm{C}\end{array}$ & 15,00 & 20,00 & 25,00 & 30,00 & 35,00 & 40,00 & 45,00 \\
\hline $\begin{array}{r}\text { gemessene } \\
\text { Wassertemperatur in }{ }^{\circ} \mathrm{C}\end{array}$ & 16,65 & 21,10 & 25,92 & 29,97 & 34,09 & 38,49 & 43,16 \\
\hline $\begin{array}{r}\text { am Thermostat eingestellte } \\
\text { Temperatur in }{ }^{\circ} \mathrm{C}\end{array}$ & 50,00 & 55,00 & 60,00 & 65,00 & 70,00 & 75,00 & 80,00 \\
\hline $\begin{array}{r}\text { gemessene } \\
\text { Wassertemperatur in }{ }^{\circ} \mathrm{C}\end{array}$ & 47,45 & 51,18 & 55,91 & 60,08 & 64,20 & 68,21 & 72,69 \\
\hline
\end{tabular}

Tabelle 3.3: Am Thermostat eingestellte und im Wasser gemessene Temperatur

schen $15^{\circ} \mathrm{C}$ und $80^{\circ} \mathrm{C}$ aufgenommen. Die dabei für das Wasser im Messvolumen der Küvette erhaltenen Temperaturen wichen von den am Thermostaten eingestellten Temperaturen ab, da die Zuleitungsschläuche der Küvette für das Temperierwasser nicht isoliert wurden, so dass eine Abstrahlung der Wärme auf dem Transportweg des Temperierwassers stattfand. Dies wurde jedoch vernachlässigt, da die eigentliche Probentemperatur direkt im zu untersuchenden Wasser bestimmt wurde und somit die am Thermostat eigestellte Temperatur lediglich ein Richtwert für die Regelung darstellte. Eine Auflistung der am Thermostat eingestellten und im Wasser gemessenen Temperaturen liefert Tabelle 3.3. Die gemessenen Wassertemperaturen sind exemplarisch in Abbildung 3.8 zusammen mit zugunsten der Übersicht ausgewählten Spektren von Wasser dargestellt. Die Spektren waren aufgrund der Veränderung des optischen Weges durch Veränderung der Temperatur teilweise stark verrauscht. Zur Entfernung der Rauschanteile in den Spektren wurden die jeweils mit Hilfe von Wavelets entrauscht. Zur Entrauschung wurde die WAVELET TOOLBox ${ }^{T M}$ von MAT$\mathrm{LAB}^{\circledR}$ in der Version 4.1 verwendet. Als Wavelet fand ein Meyer-Wavelet mit einer fünffachen Dekomposition der Spektren Verwendung. Durch diesen Schritt konnte ein Großteil des spektralen Rauschens aus den Spektren eleminiert werden. Die Justage des optischen Wegs wurde mit einem Helium-Neon-Laser durchgeführt. Hierzu wurde der Laserstrahl in die Lichtleitfaser eingekoppelt, die das anregende Licht zur Küvette leiten sollte. Die Position der Küvette wurde so eingestellt, dass der aus der Faser austretende Laserstrahl mittig und zu den Küvettenwänden parallel durch die Küvette verlief. Auf der Austrittsseite der Küvette wurde die Apertur der Detektionsfaser, die das transmittierte Licht zum Spektrometer zurückleiten sollte, so positioniert, dass der Laserstrahl wiederum möglichst gut in die Faser eingekoppelt wurde. Zur Erhöhung der Signalausbeute wurden auf beiden Seiten der Küvette (Anregungs- und Detektionsseite) NIR-transparente Linsen (Typ siehe Tabelle 3.12) verwendet. Diese Linsen wurden so justiert, dass das anregende Licht ein paralle- 


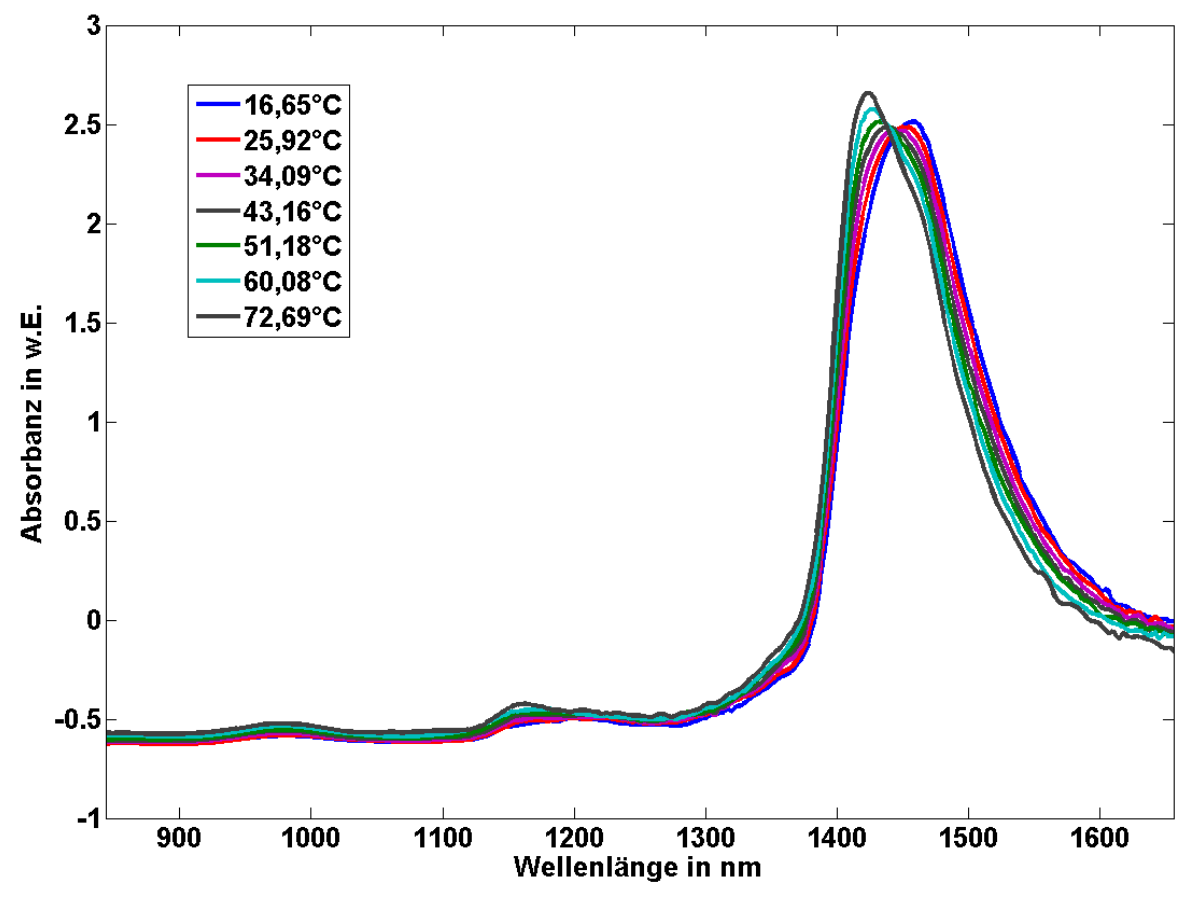

Abbildung 3.8: Ausgewählte temperaturabhängige Spektren von Wasser

les Lichtbündel ergab. Die Linse auf der Detektionsseite wurde so einjustiert, dass das spektrale Signal auf dem Spektrometerdetektor möglichst groß war und somit möglichst viel Licht von der Detektionsfaser wieder aufgenommen wurde.

\subsubsection{Aufbau zur Reflexionsmessung an Pulvern bei konstanter Materialfeuchte und variierender Temperatur}

Zur Untersuchung des Temperatureinflusses auf die Form der Wasserbande in schüttund rieselfähigen Produkten (i.d. R. Pulver) muss der Einfluss einer variierenden Probentemperatur vom Einfluss einer variierenden Materialfeuchte der Probe unterschieden werden, da sich, wie bereits erwähnt, die beiden Einflüsse in der Form der Wasserbande überlagern [54]. Daher wurde ein Versuchsaufbau (siehe Abbildung 3.9) entwickelt, der es ermöglicht, bei konstanter Materialfeuchte die Probentemperatur zu variieren, so dass die aufgenommenen Spektren lediglich noch den Beitrag der Temperaturvarianz zeigen. Die Apparatur besteht im Wesentlichen aus einem Aluminiumblock, in den Vertiefungen für die Probe eingebracht sind. Diese Probenräume lassen sich jeweils einzeln mit einer Quarzglasscheibe, die in einen Aluminiumrahmen eingeklebt ist, verschließen. Zum Einkleben der Quarzglasscheiben 


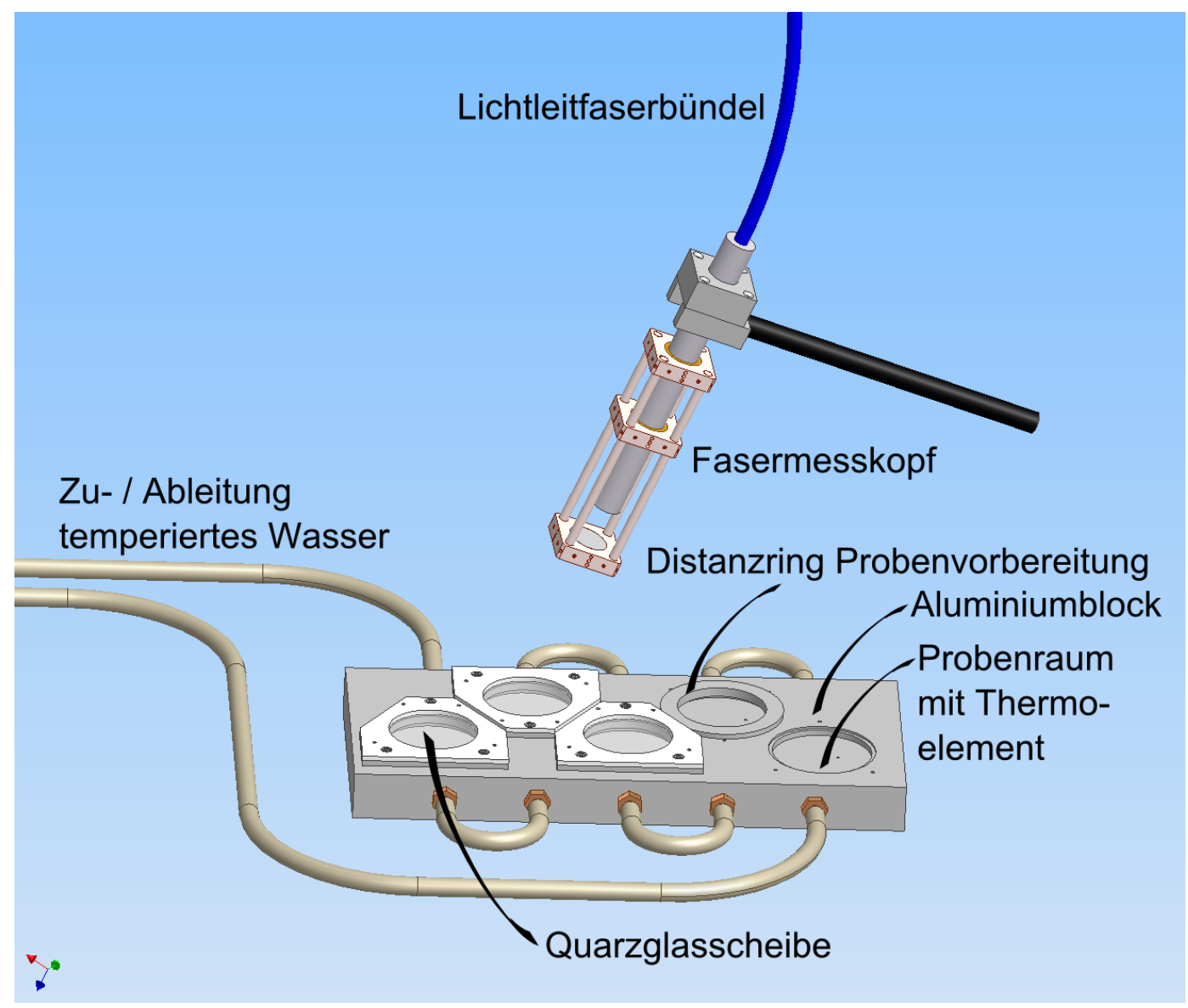

Abbildung 3.9: Versuchsaufbau zur Aufnahme der temperaturabhängigen Spektren bei konstantem Feuchtegehalt

in den Halter wurde ein bis zu einer Temperatur von ca. $150^{\circ} \mathrm{C}$ beständiger Kleber auf Silikonbasis mit dem Handelsnamen Technosil verwendet. Dieser Kleber hat den Vorteil, dass er im ausgehärteten Zustand flexibel und trotzdem ausreichend gasdicht bleibt, so dass ein Austreten der abdampfenden Materialfeuchte bei Erhöhung der Probentemperatur verhindert werden kann. Gleichzeitig wird aber auch die thermische Ausdehnung der Quarzglasscheibe und der Scheibenhalterung durch die Flexibilität des Klebers kompensiert, da es bei einem im ausgehärteten Zustand unflexiblen Kleber zum Bruch der Glasscheibe kommen kann. Der Kleber wurde im Rahmen dieser Arbeit auf die nötige Gasdichtigkeit speziell für diese Anwendung hin getestet und ausgewählt. Die jeweilige Probenkammer wurde durch Anpressen eines O-Rings mit der Aluminiumscheibenhalterung an den Aluminiumblock abgedichtet. Das Anpressen der Scheibenhalterung bewirkten jeweils drei Schrauben pro Halterung. Ein Luftvolumen im Probenraum bei eingefüllter Probe würde evtl. zu einer Änderung der Materialfeuchte der eingefüllten Probe führen, da Wasser aus 


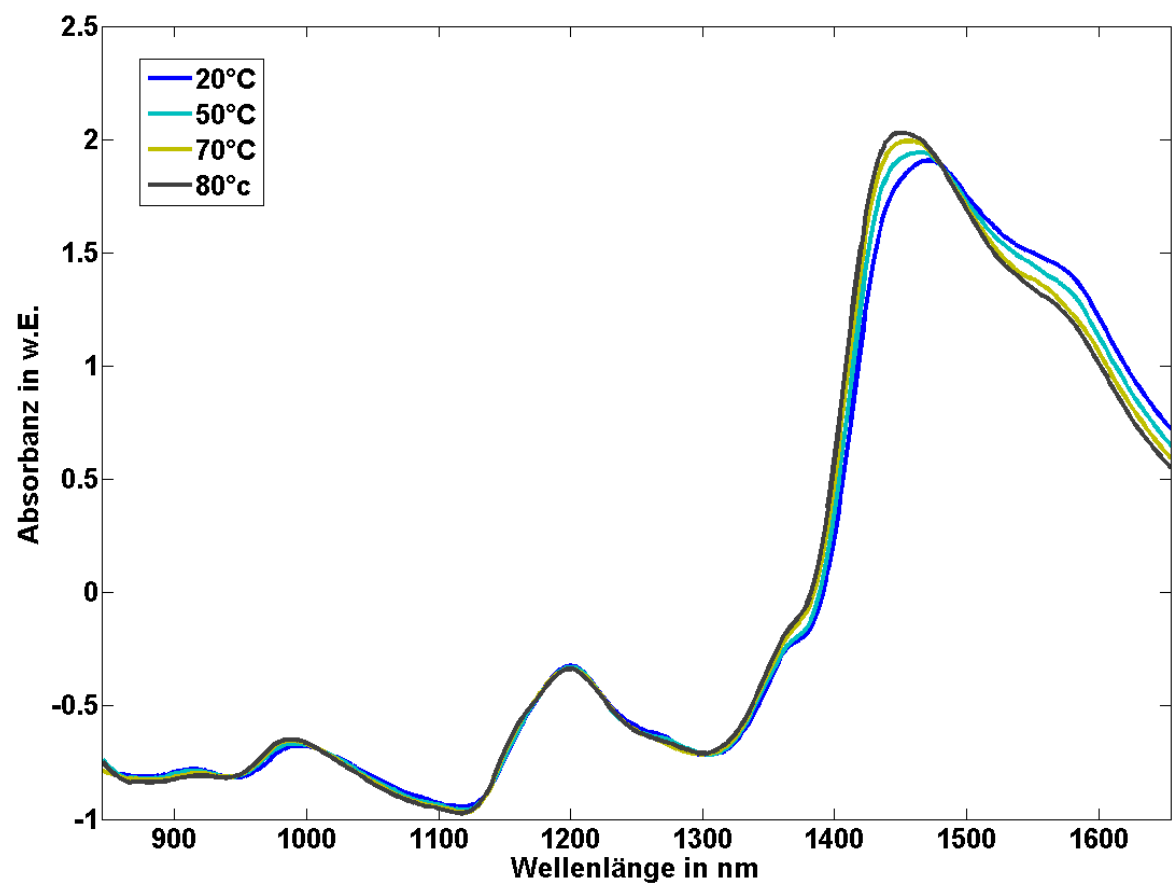

Abbildung 3.10: ausgewählte temperaturabhängige Spektren von Mehl M12 (siehe A.1) bei handelsüblicher Materialfeuchte von ca. 10,45 \% F

der Probe in den probenleeren Raum abdampfen könnte. Zusätzlich könnte ein Luftspalt zwischen Glasscheibe und Probe zu weiteren optischen Effekten führen, die die Messung verfälschen könnten. Um die Verfälschung der Messergebnisse durch diese Effekte zu verhindern, wurde ein Distanzring zur Probenvorbereitung konstruiert. Dieser Distanzring hatte die gleichen geometrischen Maße wie die Fensterhalterung mit eingeklebter Glasscheibe, so dass ein Einfüllen und Glattstreichen der Probe an der Oberkante des Distanzrings zu einer glatten Probenoberfläche führte, die somit in direktem Kontakt mit der abdeckenden Quarzglasscheibe stand. Dadurch konnten beim Verschließen des gefüllten Probenraums keine signifikanten mit Luft gefüllten Leerräume entstehen. Die Probentemperatur konnte mit diesem Aufbau variiert werden, da der Aluminiumblock jeweils unter den Probenkammern einen Temperierkanal besitzt, der mit Wasser durchspült werden kann. In Abhängigkeit von der Wassertemperatur ließ sich somit eine definierte Probentemperatur einstellen. Den Zusammenhang zwischen Temperierwasser- und Probentemperatur stellt Tabelle 3.4 beispielhaft für eine Mehlprobe dar. Die Zuverlässigkeit der spektroskopischen Messung wurde zusätzlich verbessert, indem in jede Probenkammer ein Thermoelement so eingebracht wurde, dass es die Probentemperatur messen konnte, ohne dabei die spektroskopische Messung zu beeinflussen. Desweiteren wurde nach jeder Änderung 


\begin{tabular}{|c|c|c|c|c|c|c|c|}
\hline $\begin{array}{r}\text { am Thermostat eingestellte } \\
\text { Temperatur in }{ }^{\circ} \mathrm{C}\end{array}$ & 20,00 & 30,00 & 40,00 & 50,00 & 60,00 & 70,00 & 80,00 \\
\hline $\begin{array}{r}\text { gemessene } \\
\text { Probentemperatur in }{ }^{\circ} \mathrm{C}\end{array}$ & 19,78 & 29,62 & 39,05 & 49,10 & 58,70 & 68,50 & 78,33 \\
\hline
\end{tabular}

Tabelle 3.4: Beispielhafte Darstellung des Zusammenhangs zwischen der am Thermostat eingestellten und der in der Mehlprobe M12 gemessenen Temperatur

der Zieltemperatur des Thermostaten (siehe Tabelle 3.11), der für die Regelung der Temperatur des Heizwassers zuständig war, mindestens 20 Minuten gewartet, so dass sich jeweils ein thermischer Gleichgewichtszustand einstellen konnte. Durch Beobachtung der Probentemperatur wurde jeweils der Gleichgewichtszustand festgestellt. Es wurde somit erst ein Spektrum gemessen, wenn die Probentemperatur gemessen direkt in der Probe im Rahmen der Genauigkeit der Sensoren stabil war. Eine Änderung der Materialfeuchte aufgrund der Veränderung der Probentemperatur bis $\mathrm{zu}$ einem Wert von nahezu $80^{\circ} \mathrm{C}$ wurde wie bereits erwähnt durch die Konstruktion des Aufbaus verhindert. Um trotz dieser Funktionalität eine Veränderung der Materialfeuchte auszuschließen, wurde jeweils die Feuchte für jede vermessene Probe sowohl vor dem Einfüllen als auch nach Beendigung des Temperaturmessablaufs mit Hilfe des Materialfeuchteschnellbestimmers MA100 bestimmt. Die Abweichungen zwischen den Messungen vor und nach den Temperaturuntersuchungen blieben im Bereich der Reproduzierbarkeit des Referenzmessgerätes. Kondensationen oder Ansammlungen von Wasser an den abdeckenden Quarzglasmessfenstern konnten verhindert werden, indem während der Thermostatisierungsphasen die Quarzglasfenster mit Schaumstoff abgedeckt und diese Abdeckungen jeweils nur für die eigentliche Spektrenaufnahme entfernt wurden. Als Weißreferenz wurde in diesem Fall Titandioxidpulver verwendet. Titandioxid wird aufgrund seiner Reflexionseigenschaften standardmäßig als Weißreferenzsubstanz [55] verwendet. Meist ist es wie bei SPEKTRALON ${ }^{\circledR}$, ein als Weißreferenzrohmaterial kommerziell vertriebenes Produkt, in eine Teflonmatrix eingebunden. Der Vorteil von Titandioxidpulver gegenüber einem Spektralonplättchen sind die zu Mehl ähnlichen Eigenschaften, da es sich bei beiden Proben um ein Pulver mit guten Reflexionseigenschaften im NIRSpektralbereich handelt. Außerdem wäre das Befüllen einer Temperierkammer mit einem Spektralonplättchen weitaus schwieriger gewesen, als das Einfüllen von Titandioxidpulver. Dieses Pulver wurde ebenso wie die Proben temperiert. Das Spektrum der Weißreferenz wird in der Reflektionsspektroskopie als Referenzintensität / -spektrum $I_{0}$ verwendet, so dass eine temperierte Weißreferenz die temperaturabhängigen Veränderungen, die nicht zielgrößenabhängig sind, weitestgehend eliminieren sollte. 


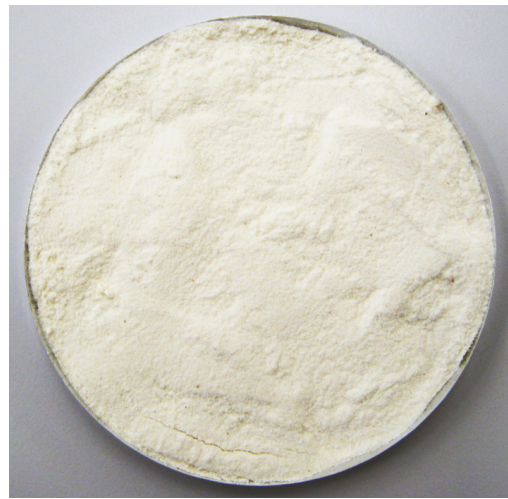

(a) Foto von Weizenmehl Type (b) Absorbanzspektrum von M18 (siehe A.1) 550

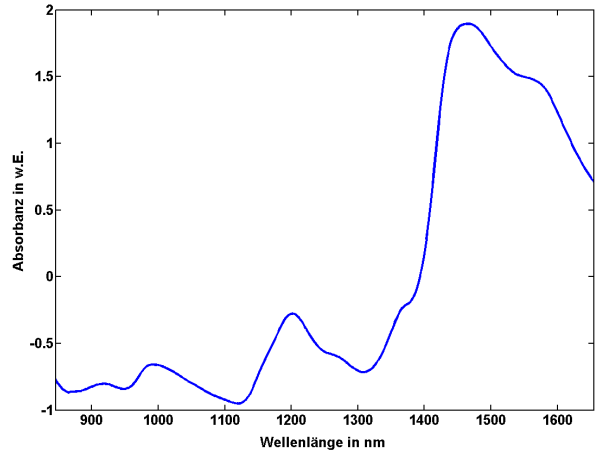

bei $20^{\circ} \mathrm{C}$

Abbildung 3.11: Absorbanzspektrum und Foto von Weizenmehl Type 550

\subsection{Auswahl der Modellsubstanz für die Untersuchungen zum Temperatureinfluss}

Zur systematischen Untersuchung des Einflusses einer variierenden Probentemperatur auf die Zuverlässigkeit einer NIR-spektroskopischen Materialfeuchtemessung musste eine Modellsubstanz gefunden werden. Im Rahmen von Untersuchungen im Vorfeld [56] und weiteren Untersuchungen im Rahmen dieser Arbeit wurden diverse Proben aus der lebensmitteltechnischen aber auch aus der Baustoff- oder der pharmazeutischen Industrie begutachtet. Diese Untersuchungen zeigten, dass Mehl als schütt- und rieselfähige Probe besonders gut als Modellsubstanz für die NIR-spektroskopischen Messungen besonders im Bereich der Lebensmittelindustrie geeignet ist. Mehl zeigt wenig Quereinflüsse, die Auswirkungen auf die spektroskopische Messung haben. So ist das Bräunen durch Erhöhung der Probentemperatur bei Mehl im Gegensatz zu z. B. Kaffeepulver (siehe Kapitel 5.1.2) vernachlässigbar. Zusätzlich lässt sich Mehl aufgrund der Qualitätsansprüche in der Lebensmittelindustrie in sehr konstanten Mahlgraden bekommen, so dass der Einfluss der Korngröße (siehe Kapitel 5.1.1) durch Variation der Mehlsorte sehr gering ist. Mehl zeigt neben der Materialfeuchtevarianz nur sehr geringe Varianzen im Spektrum, so dass Weizenmehl des Typs 550 (siehe Abbildung 3.11) aufgrund seiner guten Reflexionseigenschaften im NIR-Spektralbereich sogar als Weißreferenzstandard verwendet werden könnte, wenn die Änderung der Materialfeuchte nicht von den Umgebungsbedingungen abhängen würde. Die Änderungen der Materialfeuchte bei Mehl sind wiederum nicht so stark umgebungsabhängig wie es z. B. bei Proben von Kartoffelpüreepulver der Fall ist, da Mehl nicht sehr hygroskopisch ist. Folglich war die Handhabung der Mehlproben während des Messablaufs nicht so zeitkritisch, da sich die Materialfeuchte von Mehl eher über mehrere Minuten hinweg verändert. Im Gegensatz zu Kartoffelpüreepulver das sogar gegen Ende eines Trocknungslaufs im Materialfeuchteschnellbestimmer wieder Feuchte aus Luft aufnehmen kann. 


\subsection{Datenaufnahme, Speicherung und Verarbeitung}

Die spektralen Daten der Transmissionsmessung an reinem Wasser (Kapitel 3.3.1) und der Reflexionsmessung an Mehl- und Kaffeepulverproben (Kapitel 3.3.2, 5.1.1, $5.1 .2,5.2$, etc.) wurden zunächst von der Spektrometersoftware im sogenannten *.spc-Format, ein universelles in der Spektroskopie etabliertes Spektrenaustauschformat der Firma Thermo Fisher Scientific Inc. [57], abgespeichert. Die Spektrometersoftware wurde so eingestellt, dass die Spektren als Rohdaten in Zählereignissen (counts) des Analog-Digital-Umsetzers (ADU) auf dem Laborrechner abgespeichert wurden. Zur Korrektur der spektralen Messung auf Quereinflüsse durch Fremdlicht oder Reflexe an der Optik des Messkopfs wurden neben dem Signalspektrum der Probe und dem Weißreferenzspektrum des Titandioxids oder der Leertransmissionsmessung im Fall der Küvettenmessung auch Dunkelspektren aufgenommen, die zusätzlich analog zu den Weißreferenzmessungen als Schwarzmessungen abgelegt wurden. Ein im Rahmen dieser Arbeit erstelltes Skript ermöglichte das Einlesen der SPC-Dateien und die gleichzeitige Absorbanzberechnung mit Korrektur durch Abziehen der Schwarzspektren. Die so berechneten Absorbanzspektren wurden als „DataSetObject" in eine für MATLAB ${ }^{\circledR}$, das in dieser Arbeit in der Version 7.5.0.342 (R2007b) verwendet wurde, lesbare Datei abgespeichert. Das DataSetObject, in dieser Arbeit in Version 4.0 verwendet, ist ein freies Format ${ }^{4}$ der Firma Eigenvector Research, Inc. [58] zur Handhabung großer Datenmengen, im Speziellen abgestimmt auf die Bedürfnisse der Spektroskopie. Dieses Format ermöglicht neben der Ablage ganzer Spektrenserien auch die Zuordnung diverser Zusatzinformationen, wie Eigentümer der Spektren, technische Daten des Spektrometers, etc. Neben der Kompatibilität dieses Datenformats mit MATLAB ${ }^{\circledR}$ und der daraus resultierenden leichteren Verarbeitung der Daten, lässt es sich sehr leicht in die PLS-Toolbox der Firma Eigenvector Research, Inc., die hier in der Version 3.54 verwendet wurde, und in MATLAB ${ }^{\circledR}$ als zusätzliche Erweiterung eingebunden wird, einlesen. Die PLS-Toolbox hat bereits alle wichtigen Werkzeuge wie Datenvorbehandlungsmethoden und die Auswertung durch PLS implementiert. Zusätzlich gibt es eine grafische Oberfläche, die die Modellerstellung vereinfacht, da hier alle wichtigen Grafiken und Daten leicht zugänglich dargestellt werden können. Neben der Erstellung von Kalibriermodellen kann diese Sofware auch die Vorhersage von unbekannten Spektren anhand von fertigen Kalibriermodellen durchführen. Ein weiterer Vorteil ist die Möglichkeit, sämtliche in der grafischen Oberfläche vorhandenen Funktionen auch manuell auf der Eingabezeile von MATlaB ${ }^{\circledR}$ durchführen zu können. Dadurch konnten die entsprechenden Datenvorbehandlungen für die Auswertung in Kapitel 6.3 sinnvoll durchgeführt werden. Die fertigen Kalibriermodelle und die Vorhersagen der Materialfeuchtewerte von unbekannten Spektren konnten entsprechend wieder in MATLAB ${ }^{\circledR}$ kompatiblen Formaten abgespeichert werden.

\footnotetext{
${ }^{4}$ http://software.eigenvector.com/DataSet/index.html
} 


\subsection{Probenvorbereitung}

\begin{tabular}{|c|c|c|c|}
\hline $\begin{array}{l}\text { gesättigte wässrige } \\
\text { Lösung } \\
\text { mit viel Bodenkörper }\end{array}$ & Formel & $\begin{array}{r}\text { \% rel. Luftfeuchtigkeit } \\
\text { über der Lösung (bei } \\
20^{\circ} \mathrm{C} \text { ) }\end{array}$ & $\begin{array}{l}\text { Bezeichnung } \\
\text { der } \\
\text { Befeuch- } \\
\text { tungsart }\end{array}$ \\
\hline Natriumcarbonat & $\mathrm{Na}_{2} \mathrm{CO}_{3}$ & 92 & \\
\hline Kaliumchlorid & $\mathrm{KCl}$ & 86 & \\
\hline Natriumchlorid & $\mathrm{NaCl}$ & 76 & F76 \\
\hline Calciumnitrat & $\mathrm{Ca}\left(\mathrm{NO}_{3}\right)_{2}$ & 55 & F55 \\
\hline Kaliumcarbonat & $\mathrm{K}_{2} \mathrm{CO}_{3}$ & 45 & \\
\hline Calciumchlorid & $\mathrm{CaCl}_{2}$ & 35 & F35 \\
\hline $\begin{array}{l}\text { demineralisiertes } \\
\text { Wasser }\end{array}$ & $\mathrm{H}_{2} \mathrm{O}$ & gegen 100 & $\mathrm{H}_{2} \mathrm{O}$ \\
\hline Kieselgel & $\begin{array}{l}\text { Trockenmittel, } \\
\text { fest }\end{array}$ & gegen 0 & TRO \\
\hline $\begin{array}{l}\text { Probe aus der } \\
\text { Packung }\end{array}$ & $\begin{array}{l}\text { keine } \\
\text { Behandlung }\end{array}$ & Handelsfeuchte & DIR \\
\hline
\end{tabular}

Tabelle 3.5: Herstellung konstanter Luftfeuchtigkeit (in geschlossenen Gefäßen) [59], Beschreibung der Bezeichnungen der Befeuchtungsart, wie sie in dieser Arbeit verwendet wurden

Zur Varianz der Feuchtewerte für die in den Untersuchungen dieser Arbeit verwendeten Mehlproben mussten bestimmte Mengen des jeweils zu untersuchenden Mehls auf unterschiedliche Materialfeuchtegehalte eingestellt werden. Das Einstellen solcher Materialfeuchtegehalte wird auch als Konditionieren einer Probe bezeichnet. Zum Kondititionieren der Mehlsorten wurden jeweils ca. 20-25 g Mehl in ein dafür geeignetes Probengefäß gefüllt. Die befüllten Probengefäße wurden anschließend in luftdicht schließende Plastikschalen gestellt. Auf dem Boden dieser Plastikschalen befand sich eine gesättigte Salzlösung oder ein Trockenmittel. Die Proben wurden 24 Studen in der jeweiligen Konditionierungsschale belassen, so dass die gesamte Probe genügend Feuchte aus der umgebenden Luft aufnehmen und sich ein homogener Zustand der Feuchteverteilung im Innern der Probe einstellen konnte. In Abhängigkeit der verwendeten Salze stellte sich in den Konditionierungsgefäßen eine definierte Luftfeuchte ein, so dass sich entsprechend eine davon abhängige Ausgleichfeuchte in den Mehlproben einstellen konnte. Die unterschiedlichen Salze und die damit einhergehende Luftfeuchte über der gesättigten Lösung stellt Tabelle 3.5 dar. Nach Beendigung der Konditionierungszeit wurden die Probengefäße sofort mit einem Schraubdeckel verschlossen, so dass keine Änderung der Materialfeuchte mehr stattfinden konnte. Bevor es zu Untersuchungen an den nun konditionierten Proben kam, 
wurden die Proben in ihren Gefäßen noch einmal durch Schütteln und Bewegen der Gefäße durchmischt. Die Materialfeuchtewerte wurden für jede Probe einzeln mit dem MA100 bestimmt. Die Ergebnisse dieser Messungen sind in Tabelle $3.2 \mathrm{zu}$ finden.

\begin{tabular}{ll}
\hline Hersteller & Measurement Computing \\
Modell & USB-Temp \\
Messkanäle & 8 \\
Temperatur- & Thermoelemente (div. Ty- \\
sensoren & pen), weitere Typen mög- \\
& lich \\
Sensortyp & Thermoelement Typ K \\
\hline
\end{tabular}

Tabelle 3.6: Technische Daten des Moduls zur Temperaturaufnahme

\begin{tabular}{ll}
\hline Lieferant & Avantes \\
Typ & „low OH“ Fasern \\
Bezeichnung & FC IR400-2-ME0610152, \\
Anregungsfaser & Durchmesser 400 $\mu \mathrm{m}$ \\
Bezeichnung & FC IR600-2-ME0611069, \\
Detektionsfaser & Durchmesser $600 \mu \mathrm{m}$ \\
Faserkopplung & SMA 905 \\
\hline
\end{tabular}

Tabelle 3.7: Typen der Lichtleitfasern für die Transmissionsmessungen an Wasser 


\begin{tabular}{|c|c|}
\hline Hersteller & Frank Optic Products \\
\hline Тур & Fasermesskopf mit Y-Fasern \\
\hline Fasertyp & $\begin{array}{l}\text { Quarzglasfaser, „low } \mathrm{OH}^{\prime \prime} \text {, } \\
\text { NA } 0,22\end{array}$ \\
\hline Anregunsfasern & $\begin{array}{l}18 \text { Stück, Durchmesser } \\
320 \mu \mathrm{m}\end{array}$ \\
\hline Detektionsfaser & $\begin{array}{l}1 \text { Stück, zentral angeord- } \\
\text { net, von Anregunsfaserbün- } \\
\text { del umgeben, Durchmesser } \\
400 \mu \mathrm{m}\end{array}$ \\
\hline $\begin{array}{l}\text { Faserkopplung } \\
\text { Spektrometer- } \\
\text { seite }\end{array}$ & SMA 905 \\
\hline Faserkopplung & firmenspezifische \\
\hline Anregunsseite & $\begin{array}{l}\text { kopplung von Frank Optic } \\
\text { Products }\end{array}$ \\
\hline $\begin{array}{l}\text { Länge Faserka- } \\
\text { bel }\end{array}$ & $2 \mathrm{~m}$ \\
\hline $\begin{array}{l}\text { Reflektions- } \\
\text { messkopf }\end{array}$ & $\begin{array}{l}\text { Edelstahlrohr, Durchmesser } \\
\text { 20mm, Länge } 200 \mathrm{~mm}\end{array}$ \\
\hline
\end{tabular}

Tabelle 3.8: Technische Daten des verwendeten NIR-Fasermesskopfs

\begin{tabular}{|c|c|}
\hline $\begin{array}{l}\text { Hersteller } \\
\text { Typ } \\
\text { Verschluss }\end{array}$ & $\begin{array}{l}\text { Frank Optic Products } \\
\text { ShowTec 50S } \\
\text { mechanischer Shutter, an- } \\
\text { steuerbar durch TTL-Signal }\end{array}$ \\
\hline Leuchtmittel & \\
\hline Hersteller & Welch Allyn \\
\hline $\begin{array}{l}\text { Spannung/ } \\
\text { Strom }\end{array}$ & $12 \mathrm{~V}, 1,67 \mathrm{~A}$ \\
\hline Reflektor & $\begin{array}{l}\text { elliptischer Reflektor, Typ } \\
\text { MR11 }\end{array}$ \\
\hline Artikelnummer & 8211-002 \\
\hline Lebensdauer & ca. 2000 Stunden \\
\hline
\end{tabular}

Tabelle 3.9: Technische Daten der für die Reflektionsmessungen verwendeten NIRLichtquelle 


\begin{tabular}{ll}
\hline Hersteller & Polytec \\
Modell & PSS-L-007 \\
Typ & Wolfram-Halogen-Lampe \\
Eingangsleistung & $7 \mathrm{~W}$ \\
Farbtemperatur & $2800 \mathrm{~K}$ \\
Lebensdauer & $>2000 \mathrm{~h}$ \\
Faserkopplung & SMA 905 \\
\hline
\end{tabular}

Tabelle 3.10: Technische Daten der bei den Transmissionsmessungen verwendeten Lichtquelle

\begin{tabular}{ll}
\hline Hersteller & Contraves \\
Typ & Rheotherm 115 \\
Leistung & $1150 \mathrm{~W}$ \\
\hline
\end{tabular}

Tabelle 3.11: Thermostat zur Temperierung des Wassers und der Mehlproben

\begin{tabular}{|c|c|}
\hline Lieferant & Newport \\
\hline Тур & BCX Lens, bikonvex \\
\hline $\begin{array}{l}\text { Bestellbezeich- } \\
\text { nung }\end{array}$ & KBX049 AR1.8 \\
\hline Beschichtung & $\begin{array}{l}\text { BBAR coat } 1,0-1,5 \mu \mathrm{m} \\
\text { (Breintbandantireflexbe- } \\
\text { schichtung) }\end{array}$ \\
\hline Durchmesser & $25,4 \mathrm{~mm}$ \\
\hline Fokuslänge & $38,1 \mathrm{~mm}$ \\
\hline
\end{tabular}

Tabelle 3.12: Typen der Linsen für die Transmissionsmessungen an Wasser 


\begin{tabular}{ll}
\hline Hersteller & Polytec \\
Modell & PSS-1750 \\
Polychromator- & PSS-175, 3D-Transmissi- \\
Typ & onsgitter mit Ordnungsfilter \\
Dunkelkorrektur & interner Shutter \\
& \\
Spaltbreite & $40 \mu \mathrm{m}$ \\
Faserkopplung & SMA 905 \\
Spektralbereich & $850 \mathrm{~nm}-1650 \mathrm{~nm}$ \\
& \\
spektrale Auf- & $<4$ nm \\
lösung & \\
Streulicht & $1: 50.000$ \\
Dunkelrauschen & 5 counts \\
& \\
Lienearität & $99,9 \%$ \\
Signalrausch- & 8000 \\
verhältnis & \\
Detektor & Hamamatsu G9202-512S \\
Detektortyp & InGaAs-Diodenzeile, \\
& 512 Pixel \\
Kühlung & einstufige thermoelektrische \\
& Kühlung (TEC) \\
Datentransfer & Ethernet \\
und Steuerung & \\
Software & PSS-AXC, Version 1.8 \\
Integrationszeit & $900 \mathrm{~ms}$ \\
Mittelungszahl & 1 Spektrum \\
\hline &
\end{tabular}

Tabelle 3.13: Technische Daten des verwendeten NIR-Spektrometers 


\section{Kapitel 4}

\section{Methoden zur Datenauswertung}

\subsection{Chemometrie und multivariate Statistik}

Der Begriff Chemometrie oder Chemometrik (engl. Chemometrics) wurde 1972 von dem Schweden Svante Wold und dem Amerikaner Bruce Kowalski geprägt. Die Definition lautet [60]: Chemometrie ist die chemische Disziplin, bei der mathematische und statistische Methoden zur Planung und Auswahl optimaler Messverfahren und Experimente und zur Gewinnung maximaler chemischer Informationen bei der Analyse chemischer Daten verwendet werden.

Ein chemometrisches Werkzeug, das häufig [10, 18, 51, 61, 62] für die Auswertung von NIR-Spektren verwendet wird, ist die Partial Least Squares Regression.

\subsubsection{Partial Least Squares Regression - PLS}

Die Partial Least Squares (PLS) Methode, häufig auch Partial Least Square Regression (PLSR) genannt, ist eine Art der multivariaten ${ }^{1}$ oder auch Hauptkomponentenanalyse. Die Grundlage des PLS-Algorithmus ist die Principal Component Regression (PCR). Der Unterschied zwischen der Principal Component Regression und der Partial Least Square Regression liegt in der Art der Hauptkomponentenzerlegung, also den Kriterien, nach denen die Hauptkomponenten gesucht werden. Für eine ausführliche Erklärung der Principal Component Regression sei hier auf $[24,60]$ verwiesen. Der PLS-Algorithmus reduziert zur Erstellung einer Methode die Datenmenge auf ein Minimum. Dazu versucht er, eine Möglichkeit zu finden, spektrale Informationen aus den gemessenen Spektren, die einen höherdimensionalen Raum aufspannen, in einem Raum möglichst geringer Dimension zu beschreiben, ohne die signifikanten Informationen zu verlieren oder Daten aus dem Datensatz zu entfernen. Dies geschieht durch Transformation der spektralen Informationen in ein neues Koordinatensystem. Dazu versucht der Algorithmus über die Lösung eines Eigenwertproblems ein Koordinatensystem zu finden, bei dem alle spektralen Informationen z. B. in einer Ebene liegen (vgl. Abbildung 4.1). Somit wären die

\footnotetext{
${ }^{1}$ univariate Analyse: Verwendung einer charakteristischen Komponente; multivariate Analyse: Verwendung mehrerer charakteristischer Komponenten
} 


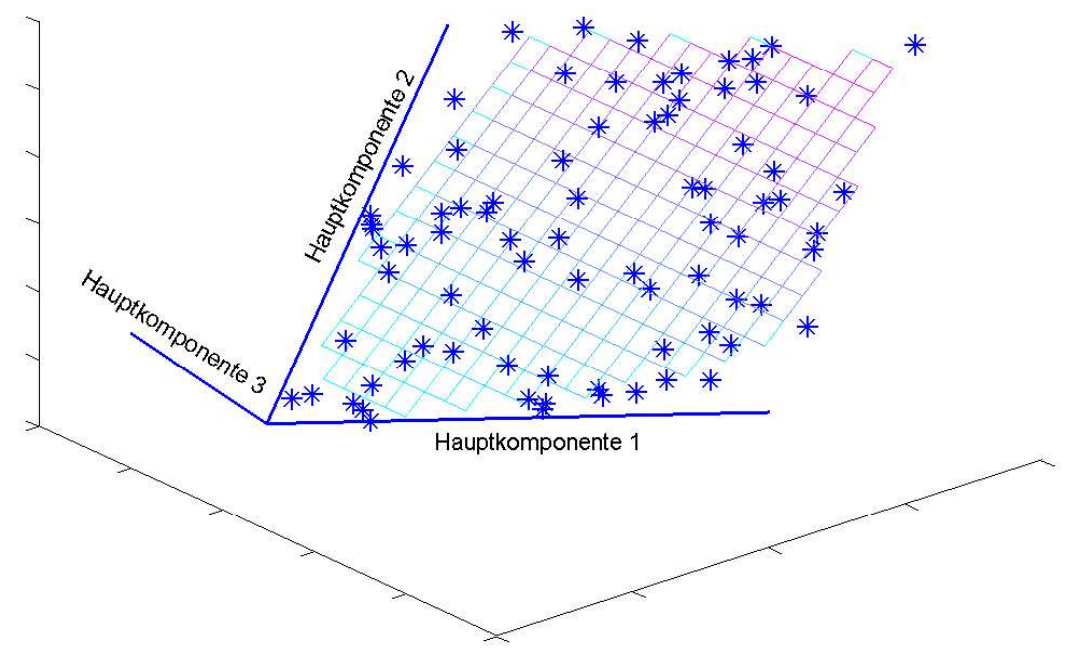

Abbildung 4.1: Dreidimensionale Darstellung der PLS-Transformation der Daten in ein neues Koordinatensystem mit 3 Hauptkomponenten (loadings), die Daten unterliegen keinem Rauschen, das Gitter soll die Fläche von Hauptkomponente 1 und 2 veranschaulichen, in die die spektralen Informationen transformiert wurden

vorher über sehr viele Dimensionen zu beschreibenden spektralen Informationen in diesem Beispiel über nur zwei Dimensionen in dieser Fläche bestimmt. Die Dimensionsreduzierung muss jedoch nicht zwangsläufig auf einen zweidimensionalen Raum hinauslaufen. Die Vektoren, die die einzelnen Koordinaten der Datenpunkte in dem neuen Koordinatensystem beschreiben, werden auch score-Vektoren oder scores ${ }^{2}$ genannt (in einem zweidimensionalen Raum werden somit zwei score-Vektoren zur Beschreibung eines Punktes benötigt). Die Achsen sind die Hauptkomponenten der Transformation und werden beim PLS auch als Rang der Berechnung bezeichnet. Die Einheitsvektoren, auch Eigenvektoren, des neuen Koordinatensystems heißen loadings oder loadings-Vektoren. Die Abweichung der spektralen Informationen aus dieser von dem PLS gefundenen Fläche (dies könnte z. B. durch Rauschen in den Messwerten bewirkt werden) würde von einer dritten Dimension beschrieben. Sollte die Abweichung aus dieser Fläche mit Hilfe der dritten Dimension nicht hinreichend

\footnotetext{
${ }^{2}$ die englischen Begriffe werden auch in deutschsprachiger Fachliteratur verwendet, die Definition der Begriffe loadings und scores wird in der Literatur nicht immer konsistent verwendet $[63,64]$
} 
genau beschrieben sein, so sucht der PLS weitere Dimensionen (höhere Ränge), bis der Fehler der Transformation auf ein Minimum reduziert ist und die spektralen Informationen mit Hilfe des neuen Koordinatensystems möglichst gut beschrieben sind. Noch genauer betrachtet versucht die Partial Least Square Regression die Kovarianz der Spektren zusammen mit der Referenzwertmatrix zu finden und bildet jede einzelne Kovarianz in einem Hauptkomponentenvektor ab.

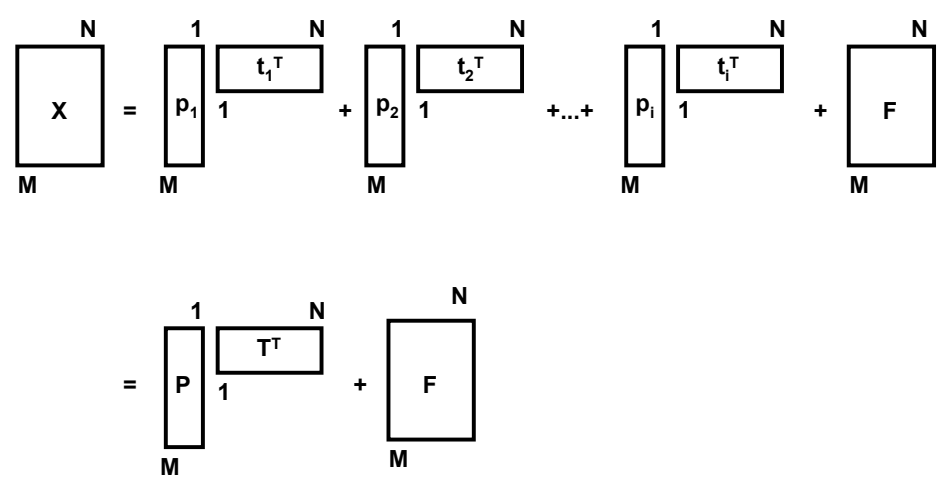

Abbildung 4.2: Blockschema der Faktorisierung der Spektraldatenmatrix

Mathematisch betrachtet, führt der PLS-Algorithmus eine Faktorzerlegung der Spektraldaten $\boldsymbol{X}$ und der Konzentrationsdaten $\boldsymbol{Y}$ durch. Dazu wird die Spektraldatenmatrix mit der Dimension $\mathrm{M} \times \mathrm{N}$, wobei $\mathrm{M}$ die Anzahl der Spektren / der Kalibrierproben und N die Anzahl der verwendeten Stützstellen / Anzahl der verwendeten Wellenlängenwerte ist, in die Vektoren $\mathbf{t}_{i}$ und $\mathbf{p}_{i}$ zerlegt. Diese Vektoren sind die loadings ${ }^{3}$ (Hauptkomponenten) $\mathbf{p}_{i}$ und die scores ${ }^{4}$ der Spektraldaten (Koordinaten der einzelnen Punkte) $\mathbf{t}_{X i}$. Der Index $i$ ist dabei die Anzahl der verwendeten Hauptkomponenten. Die Faktorzerlegung (vgl. auch Abbildung 4.2) der Spektraldaten wird beschrieben durch:

$$
\mathbf{X}=\mathbf{p}_{1} \mathbf{t}_{X 1}^{T}+\mathbf{p}_{2} \mathbf{t}_{X 2}^{T}+\mathbf{p}_{3} \mathbf{t}_{X 3}^{T}+\ldots+\mathbf{F}
$$

Dabei ist $\mathbf{X}$ die Spektraldatenmatrix. F ist die Matrix der spektralen Residuen, also der Informationen, die durch die Faktorzerlegung nicht erfasst werden können

\footnotetext{
${ }^{3} \mathbf{p}_{i}$ hat die Dimension $\mathrm{M} \times 1$

${ }^{4} \mathbf{t}_{X i}$ hat die Dimension $\mathrm{N} \times 1$
} 
(vgl. Kapitel 4.1.3). Analog zur Spektraldatenmatrix wird die Konzentrationsdatenmatrix $\mathbf{Y}$ wie folgt beschrieben:

$$
\mathbf{Y}=\mathbf{p}_{1} \mathbf{t}_{Y 1}^{T}+\mathbf{p}_{2} \mathbf{t}_{Y 2}^{T}+\mathbf{p}_{3} \mathbf{t}_{Y 3}^{T}+\ldots+\mathbf{G} .
$$

Die Konzentrationsdatenmatrix hat die Dimension $\mathrm{M} \times \mathrm{L}$, wobei L die Anzahl der zu analysierenden Stoffe in den Spektren ist (hier: $\mathrm{L}=1$, da nur Wasser, ein Stoff, analysiert wurde). Der PLS-Algorithmus verwendet die gleichen loadings-Vektoren $p_{i}$ und führt eine abhängige Faktorisierung durch, die sowohl $\mathbf{X}$ als auch $\mathbf{Y}$ gut beschreibt. Die $\mathbf{t}_{Y i}$ sind die score-Vektoren ${ }^{5}$ der Konzentrationsdaten. $\mathbf{G}$ ist die Residualmatrix der Konzentrationsdaten, also der Konzentrationsdifferenzen, die nicht von der Faktorzerlegung erfasst werden können. Die optimale Anzahl der Hauptkomponenten (des optimalen Rangs) bewirkt eine sehr gute Robustheit des PLS-Modells. Robust bedeutet in diesem Fall, dass die Analysefehler, die während der Kalibrierung ermittelt wurden, bei Analysen mit diesem Modell nicht deutlich zunehmen [63]. Die

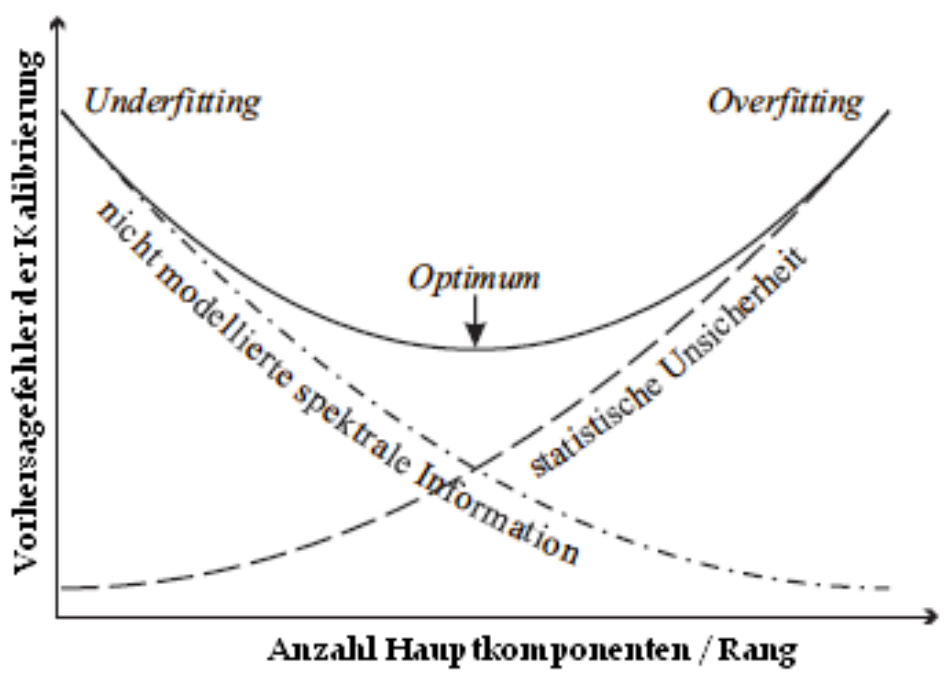

Abbildung 4.3: Abhängigkeit des Vorhersagefehlers von der Anzahl der Hauptkomponenten [63]

optimale Anzahl an Hauptkomponenten wird z. B. mit Hilfe der Kreuzvalidierung (cross validation oder ,leave one out" siehe auch Kapitel 4.1.2 und Abbildung 4.4) gesucht [24]. Neben der "leave one out Methode" können auch weitere Arten der Kreuzvalidierung zur Bewertung der optimalen Hauptkomponentenanzahl verwendet werden. Eine nähere Erläuterung hierzu ist in Kapitel 4.1.2 ausgeführt. Bei der Kreuzvalidierung nach der „leave one out Methode“ wird jeweils ein Spektrum aus dem Kalibrierdatensatz herausgenommen und mit den restlichen Spektren ein PLS-

\footnotetext{
${ }^{5} \mathbf{t}_{Y i}$ hat die Dimension $\mathrm{L} \times 1$
} 
Modell erstellt. Mit diesem Modell wird das zu bestimmende Probenmerkmal (hier die Konzentration von Wasser oder die Materialfeuchte) für das ausgelassene Spektrum und einer steigenden Anzahl an Hauptkomponenten (steigendem Rang) vorhergesagt und der Vorhersagefehler berechnet. Dies wird solange wiederholt, bis jedes Spektrum einmal ausgelassen und vorhergesagt wurde. Die Vorhersagefehlerquadrate für jedes einzelne Spektrum werden für jede geprüfte Hauptkomponentenanzahl aufsummiert und auf die Anzahl der verwendeten Spektren und zu analysierenden Komponenten in der Probe normiert. Das Minimum dieses vom Rang abhängigen Fehlers (nicht zu verwechseln mit dem $R M S E C$, vgl. Kapitel 4.1.2) gibt die optimale Anzahl an Hauptkomponenten für das PLS-Modell an. Würde bei der Modellerstellung ein Rang kleiner als der optimale Rang verwendet, so handelt es sich um ein „Underfitting“, da nicht alle für die Kalibrierung relevanten spektralen Daten modelliert würden. Würde hingegen ein zu großer Rang gewählt, spricht man vom „Overfitting“, da hier neben den für die Kalibrierung relevanten spektralen Informationen auch statistisch unsichere Daten stärker modelliert würden (vgl. Abbildung 4.3). Au§erdem würde ein zu großer Rang, also eine zu große Anzahl an Hauptkomponenten, neben der Verschlechterung des Modells auch eine größere Rechenzeit bewirken, wobei dies bei den Rechenleistungen aktueller Computer eine eher geringerer Nachteil ist.

\subsubsection{Bewertung der Güte einer Kalibrierung}

Die Güte eines mit der Partial-Least-Squares Regression erstellten Kalibriermodells kann mit Hilfe verschiedener statistischer Parameter beurteilt werden [65]:

Bestimmtheitsmaß $\boldsymbol{R}^{\mathbf{2}}$ Das Bestimmtheitsmaß $R^{2}$ gibt den aus der Regression resultierenden, prozentualen Anteil an der Varianz der Konzentration $y$ an:

$$
\begin{aligned}
R^{2} & =\left(1-\frac{S S E}{\sum_{i=1}^{m}\left(y_{i}-\bar{y}_{i}\right)^{2}}\right) \cdot 100 \% \\
\operatorname{mit} S S E & =\sum_{i=1}^{m}\left(\hat{y}_{i}-y_{i}\right)^{2} \\
R^{2} & =\left(1-\frac{\sum_{i=1}^{m}\left(\hat{y}_{i}-y_{i}\right)^{2}}{\sum_{i=1}^{m}\left(y_{i}-\bar{y}_{i}\right)^{2}}\right) \cdot 100 \% .
\end{aligned}
$$




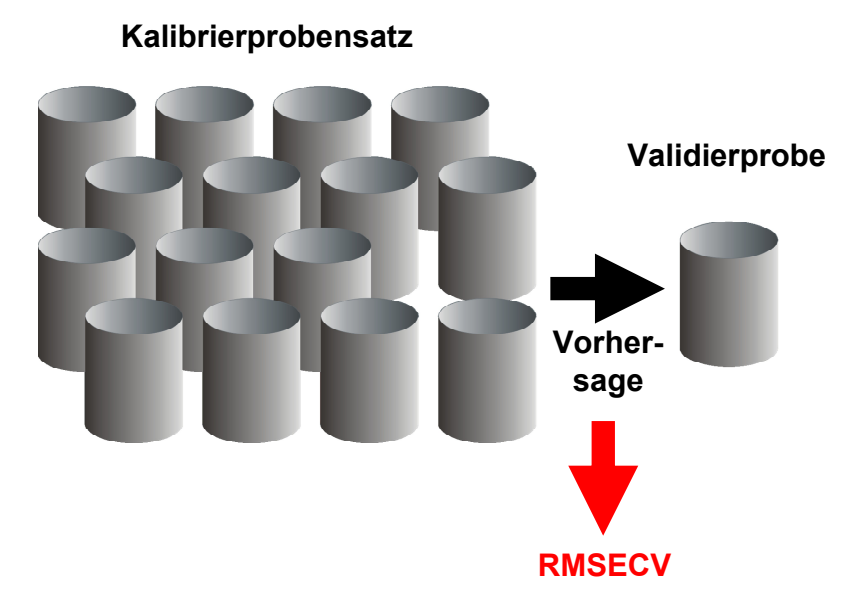

(a) Kreuzvalidierung liefert $R M S E C V$, Anwendung der Vorhersage des Modells auf Spektren aus dem Kalibrierdatensatz

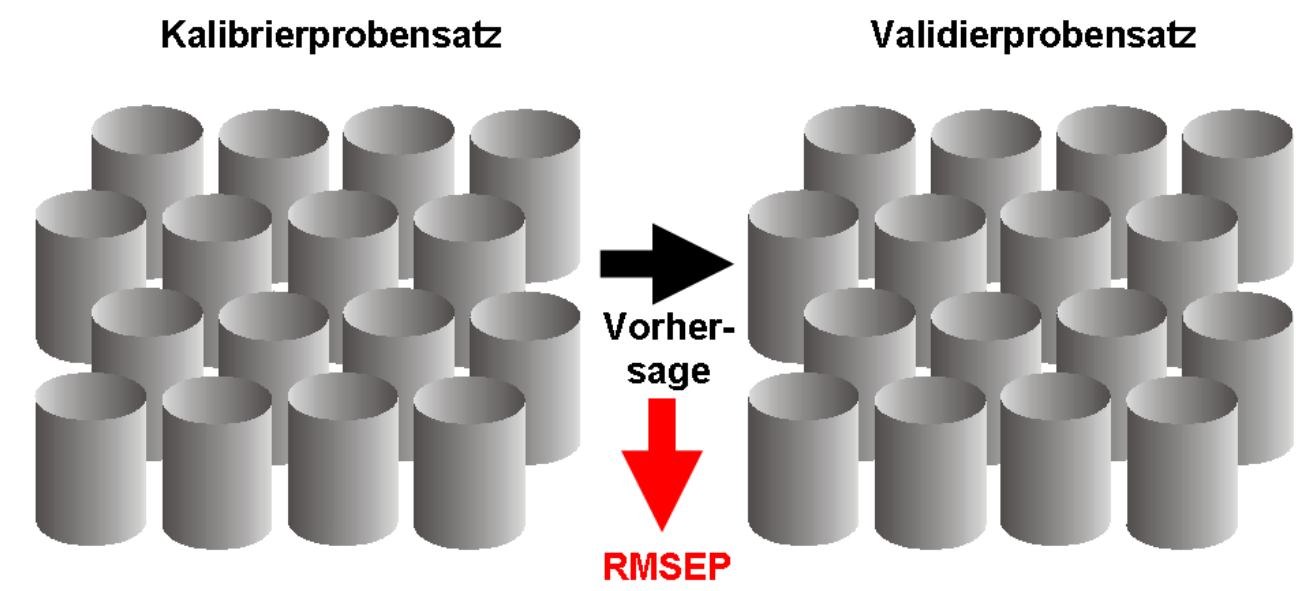

(b) Testsetvalidierung liefert RMSEP, Anwendung auf Spektren, die nicht im Kalibrierdatensatz enthalten sind

Abbildung 4.4: Kreuz- und Testsetvalidierung 
Dabei ist $m$ die Anzahl der verwendeten Kalibrierproben, $\hat{y}_{i}$ die mit dem Kalibriermodell vorhergesagte Konzentration, $y_{i}$ die mit der Referenzanalytik bestimmte Konzentration der Kalibrierproben und $\bar{y}_{i}$ der Mittelwert von $y_{i}$. Je näher $R^{2}$ gegen $100 \%$ geht, um so genauer stimmen die vorhergesagten und die mit der Referenzmethode bestimmten Konzentrationen überein. Eine Kalibrierung, die gute Analyseergebnisse liefert, zeigt einen $R^{2}>90 \%$. SSE steht für sum of squared errors und ist die Summe der Quadrate der Differenzen zwischen vorhergesagter und gemessener Konzentration. Als Grundsatz für die Bewertung eines Modells mit Hilfe des Bestimmtheitsmaßes gilt, dass der Wert $R^{2}$ stets nur im Zusammenhang mit den Voraussetzungen der Modellerstellung bewertet werden kann. Das Bestimmtheitsmaß ist nur beim Vergleich von Modellen mit gleicher Anzahl an Hauptkomponenten sinnvoll zu verwenden. Im Fall von Modellen mit unterschiedlicher Hauptkomponentenzahl kann dieser Paramter möglicherweise zu einer Fehlinterpretation führen, da er im Normalfall mit steigender Hauptkomponentenanzahl größer wird, so dass dieses Vorgehen zur Erstellung von Modellen mit einer viel zu großen Anzahl an Hauptkomponenten einhergeht. Dies führt demnach zwar zu einem vermeintlich guten und damit großen Wert für $R^{2}$, aber auch zu einem „Overfitting“ (vergleiche Abbildung 4.3 auf Seite 52) der Daten, so dass der Vorhersagefehler des Modells größer würde. Zusätzlich wird das Bestimmtheitsmaß mit steigender Anzahl an Proben auch größer werden, da der statistische Fehler $S S E$ i. d. R. mit steigender Probenanzahl kleiner wird, was wiederum zu einer Fehlinterpretation dieses Parameters führen kann (siehe auch [24]).

RMSECV Bei einer Kreuzvalidierung (Veranschaulichung siehe Abbildung 4.4(a)) wird im Fall der „leave one out Methode“ bei der Modellberechnung jeweils ein Spektrum aus dem Kalibrierdatensatz ausgelassen, ein Modell mit den übrigen Kalibrierspektren erstellt und das ausgelassene Spektrum mit diesem Modell vorhergesagt. Der Vorhersagefehler berechnet sich aus der Differenz des vom Modell vorhergesagten und des Referenzwerts. Dieser Vorgang wird so lange wiederholt bis alle Spektren einmal ausgelassen wurden. Das Quadrat des Vorhersagefehlers jeder einzelnen Iteration wird dabei aufsummiert und auf die Anzahl der verwendeten Spektren normiert. Das daraus erhaltene Fehlermaß ist der sogenannte Root Mean Square Error of Cross Validation oder auch Kreuzvaldierungsfehler:

$$
R M S E C V=\sqrt{\frac{1}{m} \cdot \sum_{i=1}^{m}\left(\hat{y}_{i}-y_{i}\right)^{2}} .
$$

Der RMSECV ist die Wurzel des mittleren Fehlerquadrats der Kreuzvalidierung. Er ist ein quantitatives Maß für die Genauigkeit, mit welcher während der Kreuzvalidierung die einzelnen Proben vorhergesagt werden. Je kleiner der $R M S E C V$ ist, um 
so genauer ist die Vorhersage jeder einzelnen Probe während der Kreuzvalidierung. Die Variablenbezeichnung ist analog zum $R^{2}$ gewählt. Die „leave one out Methode“ sollte jedoch nur für Kalibrierdatensätze mit wenigen Spektren (ca. 20-30 Spektren) verwendet werden, da sonst der $R M S E C V$ leicht misverstanden werden kann, da er bei großer Population und somit großer Varianz im Spektraldatensatz kleiner sein könnte, als es die Robustheit des Modells wirklich erlauben würde. Im Fall größerer Datensätze können auch andere Kreuzvalidierungsmethoden, wie z. B. die „leave one out Methode“ mit mehr als einer Probe je Iterationsschritt oder die „random subsets Methode", die den Kalibrierdatensatz zufällig in Teildatensätze unterteilt und dann wie die ,leave one out Methode“ mit diesen Unterdatensätzen verfährt, verwendet werden, um an dieser Stelle nur zwei weitere Beipiele zu geben. Weiterführende Informationen zur Kreuzvalidierung können in [24, 66] gefunden werden.

RMSEP / RMSEE Der RMSEP ist der mittlere Fehler, den das Kalibriermodell bewirkt, wenn ein Validierdatensatz vorhergesagt wird (Veranschaulichung siehe Abbildung 4.4(b)). Die Abkürzung RMSEP steht dabei für Root Mean Square Error of Prediction:

$$
R M S E P=\sqrt{\frac{1}{m} \cdot \sum_{i=1}^{m}\left(\hat{y}_{i}-y_{i}\right)^{2}} .
$$

Er berechnet sich aus dem mittleren Fehlerquadrat $\left(\frac{S S E}{m}\right)$ des Vorhersagewerts $\hat{y}_{i}$ gegenüber dem Referenzwert $y_{i}$. Eng verwandt mit dem $R M S E P$ ist der RMSEE, der Root Mean Square Error of Estimate. Er gibt den modellinhärenten Fehler der Kalibrierung an. Zur Berechnung dieses Werts werden die Kalibrierspektren mit dem Modell vorhergesagt und genauso wie die Validierspektren mit den zugehörigen Referenzwerten verglichen. Folglich wird in die Gleichung 4.7 für $\hat{y}_{i}$ die Vorhersagewerte der Kalibrierspektren und für $y_{i}$ die zugehörigen Referenzwerte eingesetzt, um den $R M S E P$ zu erhalten.

\subsubsection{Ausreißererkennung}

Die Erkennung von Ausreißern spielt zum Einen eine wichtige Rolle bei der Entwicklung eines zuverlässigen Modells und zum Anderen bei der Sicherheit einer Analyse. Ein Ausreißer im Kalibrierdatensatz kann die Analyse unbekannter Proben verfälschen, so dass eine sichere Vorhersage der Konzentration nicht mehr gewährleistet ist. Auch ein Ausreißer in der Gruppe der zu analysierenden Proben wird ein unsicheres Ergebniss in der Vorhersage bewirken. Es ist daher sinnvoll zu überprüfen, wie gut ein Spektrum, sei es das einer unbekannten oder einer Kalibrierprobe, zu den Kalibrierspektren „passt“. Auch ein Fehler in den Referenzwerten, sei es durch falsche Referenzanalytik oder lediglich der fehlerhaften Übetragung der Referenzwerte, wird einen Einfluss auf das zu entwickelnde Modell haben. Andererseits muss 
ein Ausreißer nicht zwingend auch ein fehlerhafter Datensatz im Sinne der zuverlässigen Bestimmung eines oder mehrer Analytenkonzentrationen sein. Es ist durchaus auch möglich, dass ein Datensatz aus Spektrum und zugehörigen Referenzwert(en) zwar im Sinne der Kalibrierung zunächst als Ausreißer zu klassifizieren ist. Jedoch kann es sein, dass genau eine solche Probe repräsentativ für eine Änderung in den zu analysierenden Proben wie z. B. einem neuen Erntejahr ist, so dass diese Probe nicht verworfen sondern mit Hilfe weiterer in dieser Varianz typischen Proben zusätzlich in den Kalibrierdatensatz aufgenommen werden, um ein neues Modell, das gegen diese Varianz robuster ist, zu erstellen. Bei der Ausreißerdiagnostik werden dieselben Maße verwendet, die auch in der Clusteranalyse zur Anwendung kommen. Die Clusteranalyse [60, 24] hat im Gegensatz zur Ausreißerdiagnostik das Ziel, mögliche Gruppierungen von Unterdatensätzen in einem großen Datensatz zu finden. Diese können dann zu einzelnen Datensätzen aus dem Gesamtdatensatz extrahiert und zu Modellen zusammengefasst werden. Die Maße und Parameter der Ausreißerdiagnostik und der Clusteranalyse werden im Folgenden erläutert, jedoch lassen sich für diese Maße keine Richt- oder Maximalwerte angeben, da diese stark von dem Modell und den vom Modell dargestellten Varianzen abhängig sind.

\section{Euklidische Distanz}

Zur Klassifizierung von Spektren und der Feststellung ihrer sicheren Vorhersagbarkeit oder der Zuordnung in einen Kalibrierdatensatz können verschiedene Distanzmaße verwendet werden. Ein grundlegendes Maß, die Distanz zwischen zwei Spektren im Spektraldatenraum festzustellen, ist die Euklidische Distanz

$$
d_{i}=\left[\sum_{k=1}^{K}\left(x_{i k}-\bar{x}_{k}\right)^{2}\right]^{1 / 2}
$$

wobei $K$ die Anzahl der spektralen Stützstellen des Spektrums $x_{i}$ ist. $x_{i k}$ sind somit die Stützstellen des jeweiligen Spektrums. $\bar{x}_{k}$ sind die Stützstellen des Mittelwertspektrums aller Spektren. Die Euklidische Distanz gewichtet alle Richtungen im Spektralraum gleich. Demnach liegen Spektren mit gleicher Euklidischer Distanz auf konzentrischen Kreisen um das Mittelwertspektrum $\bar{x}$ (siehe Abbilung 4.5). Ein Alternative zu der Euklidischen Distanz ist die Mahalanobisdistanz, die die Hauptkomponenten im Zusammenhang mit der von ihnen modellierten Varianz gewichtet.

\section{Mahalanobisdistanz}

Die Mahalanobisdistanz ist ein quantitatives Maß für die Erkennung von Ausreißern. Die Berechnung der Mahalanobisdistanz ermöglicht eine Aussage darüber, wie gut das Spektrum einer Probe zu den Spektren des Kalibrierdatensatzes ,passt“. Sie ist definiert als die richtungs- und somit varianzabhängige Differenz aus dem tatsächlich 


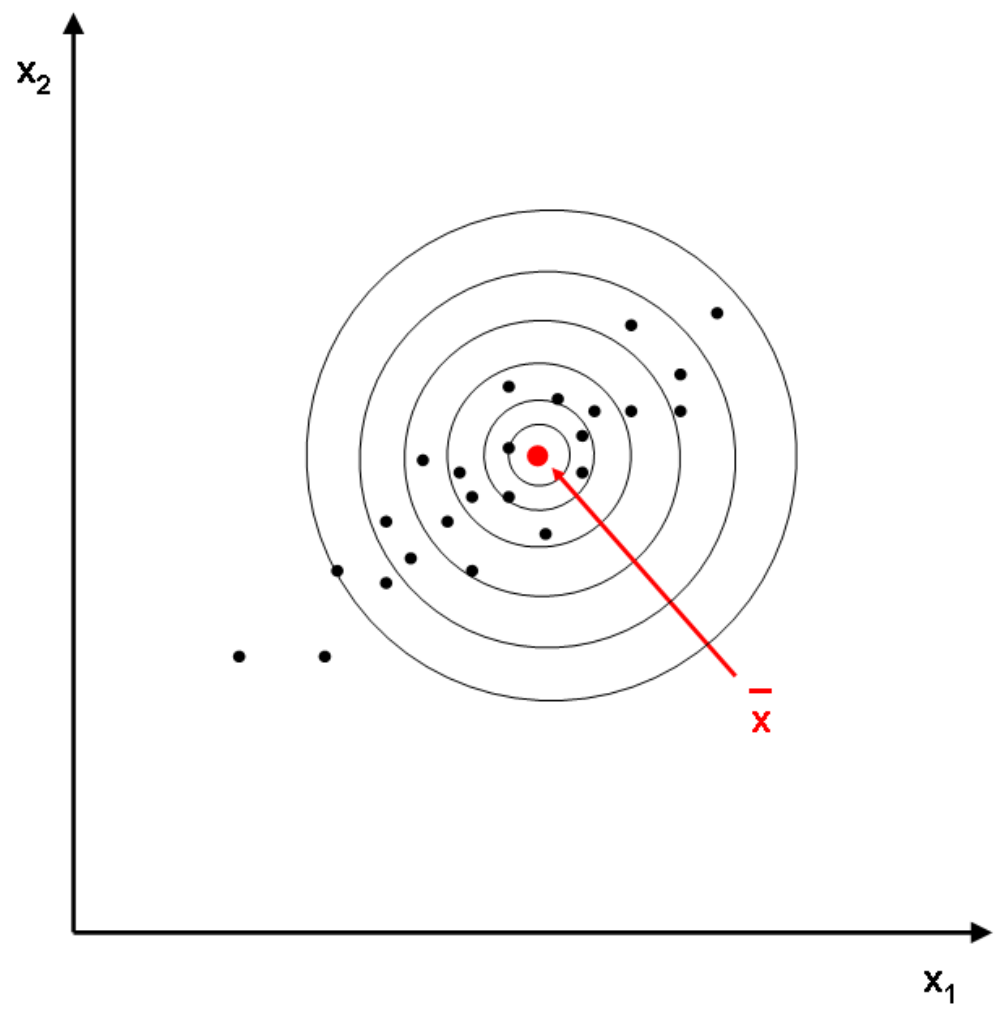

Abbildung 4.5: Die Euklidische Distanz gibt den richtungsunabhängigen Abstand eines Spektrums zum Mittelwert aller Datensätze $\overline{\mathbf{x}}$ an, hier veranschaulicht an zwei Wellenlängenwerten

gemessenen Spektrum der Probe und dem Mittelwertspektrum aller Spektren des Kalibrierdatensatzes [67] (siehe Abbildung 4.7) und wird mathematisch beschrieben mit

$$
d_{i}=\left[\sum_{a=1}^{A}\left(\hat{t}_{a}-\hat{t}_{p a}\right)^{2} / \hat{\lambda}\right]^{1 / 2}
$$

wobei $\hat{t}_{a}$ der $a$-te Hauptkomponentenscorevektor für das zu untersuchende Spektrum, $\hat{t}_{p a}$ in diesem Fall der entsprechende Vektor für das Mittelwertspektrum und $\hat{\lambda}$ der Eigenwert der Hauptkomponente $a$ ist. Je größer die Differenz des gemessenen Spektrums zu dem Mittelwertspektrum der Kalibrierspektren ist, um so mehr wächst der Wert der Mahalanobisdistanz an. Der Distanzwert wächst demnach auch an, wenn die Komponentenkonzentrationen einer untersuchten Probe höher sind als der Kalibrierbereich des Modells und daher die Analyten-Spektren stärker von dem Mittelwertspektrum abweichen. Die Mahalanobisdistanz ist daher ein quantitatives Maß für die Zuverlässigkeit der Analyse. Die Mahalanobisdistanz ist sehr eng verwandt mit dem Hebel (leverage) eines Spektrums (siehe auch Kapitel 4.1.3). Ein Maß für die Größe der Mahalanobisdistanz, wann ein Spektrum ein Ausreißer ist, 
wird an dieser Stelle nicht weiter betrachtet, da in der NIR-Spektroskopie standardmäßig der Hebel zur Erkennung von Ausreißern mit großem Einfluss auf das Modell verwendet wird. Eine Daumenregel für die Größe der Mahalanobisdistanz zur Charakterisierung eines Ausreißers liefert die Arbeit von Whitfield et. al. [68].

\section{Ausreißerarten}

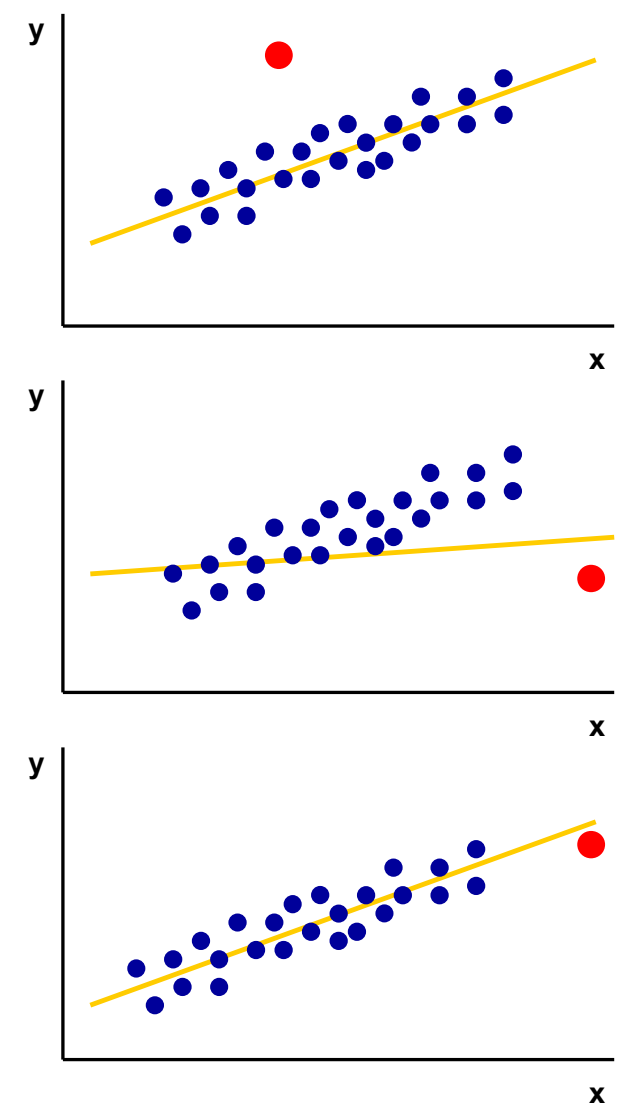

Abbildung 4.6: Einfluss unterschiedlicher Ausreißerarten, oben: $y$-Ausreißer (Referenzwertausreißer), mitte: $x / y$-Ausreißer (spektral und im Referenzwert auffällig), unten: $x$-Ausreißer (spektrale Ausreißer)

Es gibt prinzipiell zwei unterschiedliche Situationen, in denen Ausreißer auftreten können. Diese Fälle müssen unterschieden werden, da sie unterschiedliche Auswirkungen auf ein Kalibriermodell oder die Vorhersage eines solchen Modells haben. Es 
handelt sich dabei um Kalibrier- und Vorhersageausreißer. Kalibrierausreißer sind Ausreißer, die bereits im Kalibrierdatensatz enthalten sind und somit bereits einen Einfluss auf die Modellrobustheit haben. Werden sie unbeachtet im Kalibrierdatensatz belassen, können sie das gesamte Modell derart verfälschen, dass sich ihre Existenz auf zukünftige Vorhersagen signifikant auswirkt. Ein Vorhersageausreißer wiederum entsteht erst in der Phase nach der Erstellung eines Kalibriermodells. Er hat somit keine Auswirkungen auf die Robustheit und die generelle Gültigkeit eines Kalibriermodells. Ein solcher Ausreißer kann aber ein Hinweis auf fehlende Varianzmodellierung im Kalibrierdatensatz sein. In diesem Fall müsste der Kalibrierdatensatz um diese und weitere in ihrem Varianzbeitrag ähnliche Proben erweitert und ein neues Modell erstellt werden. Aufgrund der Nomenklatur werden spektrale Ausreißer auch als $x$-Ausreißer und Ausreißer in der Referenzwertmatrix $y$ auch als $y$-Ausreißer bezeichnet. Spektrale Ausreißer sind dabei Spektren, die nicht zu der Grundgesamtheit des Kalibrierdatensatzes, also der $x$-Datenmatrix, passen. So können z. B. aus Versehen Spektren von falschen Proben einem Kalibrierdatensatz hinzugefügt worden sein. Ebenso können auch Vorhersageausreißer spektraler Natur sein, wenn das Spektrum nicht zur Kalibrierung passt. Referenzwert- oder $y$-Ausreißer sind Datenpaare, bei denen die $y$-Werte eine andere Beziehung zu den $x$-Daten als die sonstigen Referenzwerte zu den jeweiligen Spektren des Kalibrierdatensatzes zeigen. $y$-Ausreißer enstehen z.B. durch Fehler in der Referenzanalytik oder beim Übertragen der Referenzwerte. Diese Ausreißerart kann nur im Kalibrierdatensatz gefunden werden, da die Referenzdaten in der Vorhersagephase nicht zur Verfügung stehen. Die Abbildung 4.6 veranschaulicht die Auswirkung der unterschiedlichen Ausreißertypen auf ein lineares Modell. Zum besseren Verständniss werden die $x$ Daten nur eindimensional dargestellt. Im oberen Abbildungsteil ist ein $y$-Ausreißer gezeigt. Es ist leicht zu erkennen, dass ein solcher Ausreißer bei einem großen Basisdatensatz einen sehr geringen Einfluss auf das Modell hat. Die mittige Darstellung zeigt den Einfluss eines $x$ - und $y$-Ausreißers auf das Kalibriermodell. Hier lässt sich gut beobachten, dass ein solcher Ausreißer das gesamte Modell in seinem Verhalten stark verzerren kann. Im unteren Teil der Abbildung ist ein $x$-Ausreißer dargestellt, der allerdings sehr nah an der Regressiongeraden liegt. Aus diesem Grund würde ein solcher Ausreißer einen sehr geringen Effekt auf das Modell haben. Cook und Weisberg haben in Ihrer Arbeit [69] gezeigt, dass eine Probe dann starken Einfluss auf das Kalibriermodell zeigt, wenn sie sowohl einen bestimmten Mindestabstand vom Mittelwert in $x$ als auch einen großen Abstand zu der linearen Regression des Datensatzes aufweist. Bei einer solchen eindimensionalen Zuordnung sind die Ausreißer leicht zu bestimmen. Wird eine mehrdimensionale Zuordnung benötigt, wie z. B. ein Spektrum mit 512 Stützstellen zu einem Referenzwert der Materialfeuchte, könnte die Betrachtung von Ausreißern für jeden Kanal einzeln, wie für die eindimensionale Zuordnung erörtert, durchgeführt werden. Dies würde jedoch keine Korrelationen innerhalb der Spektren berücksichtigen. Die sinnvolleren Methoden zur Ausreißeranalytik sind multivariater Natur und werden im Folgenden beschrieben. 


\section{Spektrale Ausreißer}

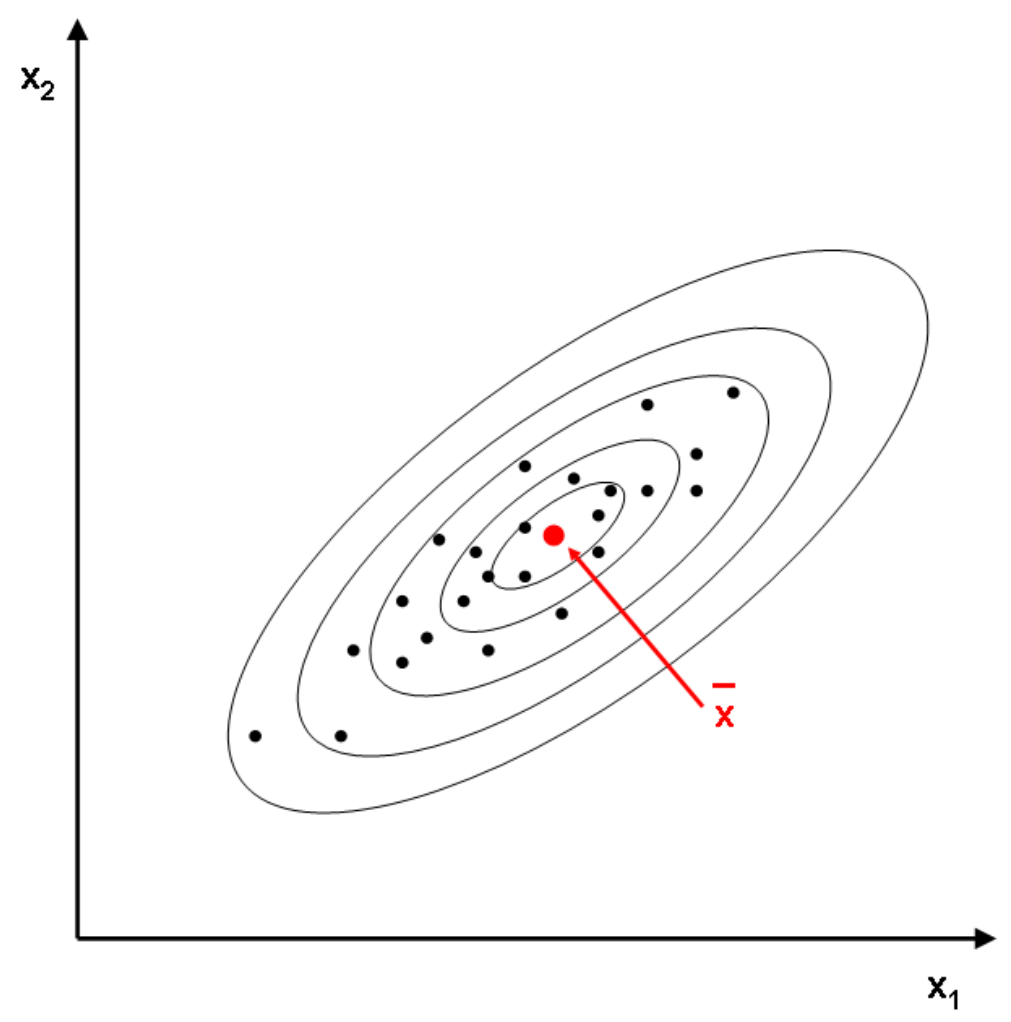

Abbildung 4.7: Punkte mit gleichem Hebel liegen auf einer Elipse mit Zentrum auf dem Mittelwert aller Datensätze $\overline{\mathbf{x}}$. Der Hebel ist eng verwandt mit der Mahalanobisdistanz, daher gilt diese Abbildung analog auch für dieses Distanzmaß

Der wichtigste Kennwert zur Klassifizierung eines spektralen oder $x$-Ausreißers ist der Hebel (engl. leverage) $h_{i}$ [69]. Ein solcher Ausreißer wird auch als H-Ausreißer bezeichnet. Der Hebel ist ein Maß für den Einfluss eines Datensatzes auf das gesamte Kalibriermodell. Je größer der Hebel, um so größer ist auch die Verzerrung des Modells bei Berücksichtigung dieses Datensatzes. Dieser Kennwert ist sehr eng verwandt (siehe Gleichung 4.12) mit der Mahalanobisdistanz $d_{i}$ (siehe Kapitel 4.1.3 auf Seite 57) und ist für ein Spektrum $i$ im Zusammenhang mit der MLR (multiple lineare Regression, näheres siehe [24,60]) definiert als

$$
h_{i}=\mathbf{x}_{i}^{t}\left(\mathbf{X}^{t} \mathbf{X}\right)^{-1} \mathbf{x}_{i},
$$

wobei $\mathbf{X}$ die Matrix der zentrierten $x$-Kalibrierdaten und $\mathbf{x}_{i}$ der zentrierte $\mathbf{x}$-Vektor für das Spektrum $i$ ist. Sind die Spektraldaten nicht zentriert, so kommt noch ein 
Achsenabschnittsterm 1/N der linearen Regression hinzu

$$
h_{i}=\frac{1}{N}+\mathbf{x}_{i}^{t}\left(\mathbf{X}^{t} \mathbf{X}\right)^{-1} \mathbf{x}_{i}
$$

wobei $\mathrm{N}$ die Anzahl der Proben ist. Spektren mit gleichem Hebel liegen geometrisch betrachtet im Spektraldatenraum auf einem Ellipsoid um den Mittelwert $\overline{\mathbf{x}}$ aller Spektren (siehe Abbildung 4.7). Die Form dieses Ellipsoids ist nach Gleichung 4.10 für $h_{i}=$ const. festgelegt durch $\mathbf{X}^{t} \mathbf{X}$, da $\mathbf{x}_{i}$ das jeweilige Spektrum darstellt. Ein Datensatz muss demnach einen größeren Euklidischen Abstand vom Zentrum zeigen, wenn er in einer Richtung größerer Varianz liegt als ein Datensatz, der sich in Richtung geringerer Varianz einordnet, damit diese den gleichen Hebelwert haben. Wird der Hebel wie hier als Distanzmaß im Spektraldatenraum verstanden, so kann er sowohl Ausreißer im Kalibrierdatensatz als auch bei Vorhersagen klassifizieren. Wird der Hebel für ein PCR- oder PLS-Modell bestimmt, so wird $h_{i}$ normalerweise für die score-Vektoren $t_{i}$ berechnet. Der Hebel wird demnach nicht mehr im Spektralsondern im score-Datenraum berechnet. Die Gleichung dafür lautet

$$
h_{i}=\frac{1}{N}+\sum_{a=1}^{A} \frac{\hat{\mathbf{t}}_{i a}^{2}}{\hat{\lambda}_{a}}=\frac{1}{N}+d_{i}^{2},
$$

wobei $\hat{t}_{i a}$ der score-Wert, $\hat{\lambda}_{a}$ die Summe der quadrierten score-Werte in Richtung der Hauptkomponente $a$ für $A$ Hauptkomponenten und Spektrum $i$ und $d_{i}$ die Mahalanobisdistanz (siehe Kapitel 4.1.3 auf Seite 57) ist. Unter bestimmten Bedingungen kann es sogar sein, dass eine Probe in einer bestimmten Varianz-, also einer Hauptkomponentenrichtung, einen sehr großen Hebel aufweist. Dies kann ein Hinweis darauf sein, dass diese Hauptkomponente sogar einzig für die Modellierung dieser Probe benötigt wird. Würde diese Probe aus dem Kalibrierdatensatz entfernt, würde die zugehörige Hauptkomponente nicht mehr in der Kalibrierung auftauchen. Ein genaues Maß für die Größe des Hebelwertes, wann ein Spektrum als H-Ausreißer zu betrachten ist, kann nicht festgelegt werden, da dies wiederum von der jeweiligen Probe abhängig ist. Eine Daumenregel für dieses Problem haben Velleman und Welch aufgestellt [70]. Demnach sollte ein Wert des zwei- bis dreifachen von $(1+A) / N$ ein Warnhinweis sein, dass eine Probe einen Ausreißer darstellt. Ein Spektrum mit einem hohen Hebelwert hat zwar zunächst einen großen Einfluss auf das Kalibriermodell, jedoch kann dies wiederum auch der Hinweis auf eine für die Modellierung signifikante Probe sein, so dass diese Probe nicht aus dem Datensatz entfernt werden sollte, sondern sogar weitere Proben mit ähnlichen Varianzbeiträgen hinzugefügt werden müssen. Ob eine solche Probe nun als Ausreißer aus dem Datensatz entfernt werden sollte, oder sogar aufgrund ihrer Signifikanz für eine neue Varianz im Modell mit zusätzlicher Erweiterung dieser Varianz durch weitere Proben bleiben sollte, muss der Chemometriker anhand seiner Erfahrung bei der Betrachtung des Datensatzes und der zugehörigen Randbedingungen des Probenmaterials entscheiden! 


\section{Referenzwert- und spektrale Residuen}

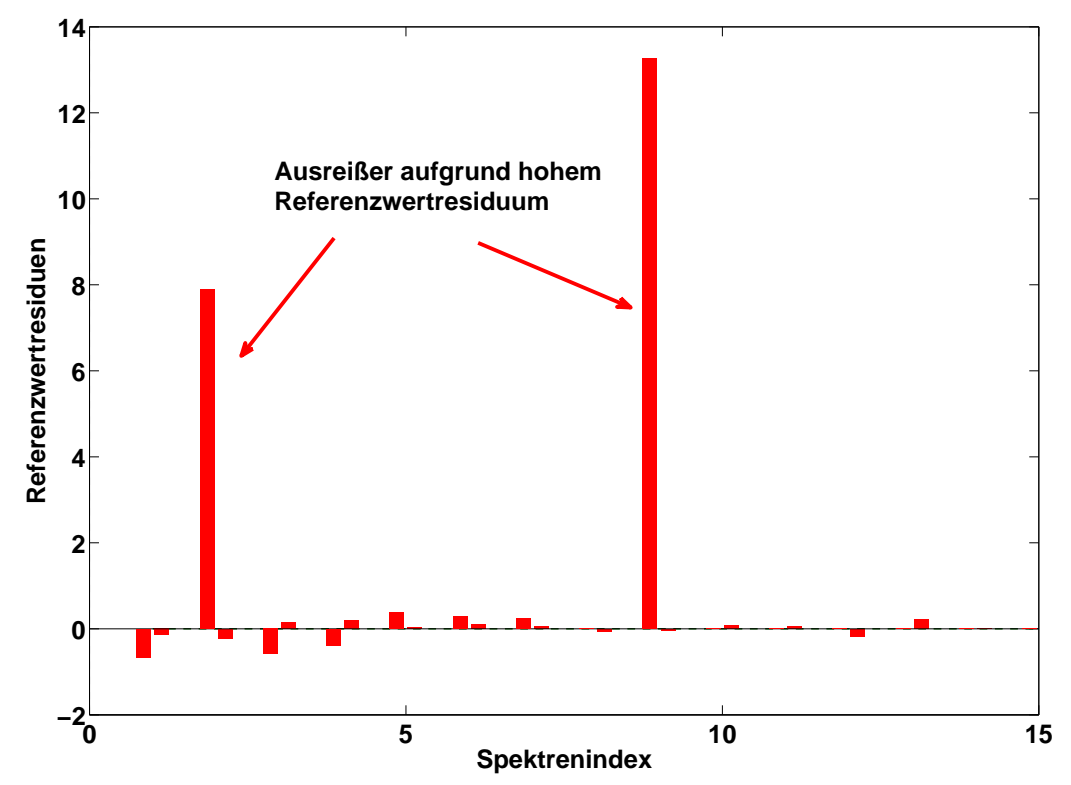

Abbildung 4.8: Ausreißer in den Referenzwertresiduen

Eine weitere Möglichkeit zur Erkennung von Ausreißern ist die Bestimmung der Residuen. Die Referenzwertresiduen $\hat{f}_{i}$ (siehe Abbildung 4.8) für eine Probe $i$ sind allgemein betrachtet die Differenz zwischen dem beobachteten / gemessenen Wert $y_{i}$ und dem mit dem Modell geschätzten Wert $\hat{y}_{i}[60]$. Sie berechnen sich nach

$$
\hat{f}_{i}=y_{i}-\hat{y}_{i}=y_{i}-\bar{y}-\hat{\mathbf{t}}_{i}^{t} \hat{\mathbf{q}}
$$

wobei $\hat{\mathbf{t}}_{i}$ der jeweilige score-Vektor und $\hat{\mathbf{q}}$ der Vektor der $y$-loadings ist. Der obere Teil der Abbildung 4.6 veranschaulicht ein $y$-Residuum. Dabei ist die Ausgleichsgerade das Regressionsmodell und der Abstand des Ausreißers in $y$-Richtung das Residuum für diesen Vorhersagewert. Eine genaue Regel, wie groß ein $y$-Residuum sein darf, um einen Ausreißer zu klassifizieren, existiert nicht. Martens und Næs [71] haben als Daumenregel eine Annäherung für einen Warnhinweis, ab dem ein Residuum auf einen Ausreißer hindeutet, vorgeschlagen. Demnach können Werte mit einem $y$-Residuum größer oder gleich dem 2 bis 3 fachen des Vorhersagefehlers RMSEE (siehe Kapitel 4.1.2) Ausreißer sein.

Die spektralen Residuen $\hat{\mathbf{e}}_{i}$ (siehe Abbildung 4.9) sind als die Differenz zwischen gemessenem Spektrum $\mathbf{x}_{i}$ und von dem Modell erwarteten Spektrum $\hat{\mathbf{x}}_{i}$ definiert. Der Residuenvektor $\hat{\mathbf{e}}_{i}$ beinhaltet somit die elementweise Differenz aus gemessenem und erwartetem Spektrum und berechnet sich nach

$$
\hat{\mathbf{e}}_{i}^{t}=\mathbf{x}_{i}^{t}-\hat{\mathbf{x}}_{i}^{t}=\mathbf{x}_{i}^{t}-\hat{\mathbf{t}}_{i}^{t} \hat{\mathbf{P}}^{t}
$$




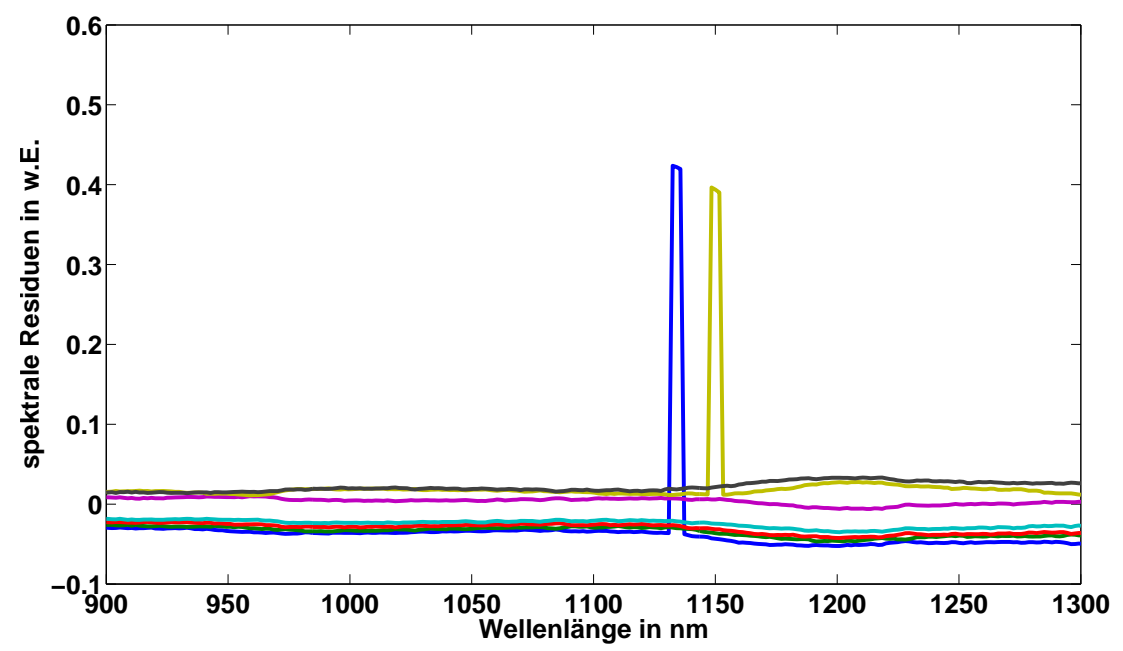

Abbildung 4.9: Ausreißer in den spektralen Residuen, die beiden auffälligen Spektren wurden künstlich mit Pixelfehlern belegt

Je größer das spektrale Residuum für eine Probe ist, d.h. je größer die Differenz zwischen dem tatsächlichen und dem vom Modell erwarteten Spektrum ist, um so wahrscheinlicher ist diese Probe ein Ausreißer. Bewertet wird eine Probe als Ausreißer in Abhängigkeit von den spektralen Residuen, die bei der Modellbildung für jedes einzelne Kalibrierspektrum und dem dazugehörigen Vorhersagespektrum berechnet werden. Demnach ist ein zu analysierendes Spektrum als Ausreißer zu bewerten, wenn die dazugehörigen Residuen stark von den Residuen des Modells abweichen. Ein expliziter Wert ist an dieser Stelle jedoch nicht sinnvoll anzugeben, da der jeweilige Maximalwert stark von dem verwendeten Modell und dem darin modellierten Varianzen abhängt. Die Höhe eines solchen Maximalwertes sinnvoll festzulegen unterliegt der Erfahrung des Chemometrikers. Ein Hilfsmittel zur Bewertung der spektralen Residuen ist die Darstellung des Residuenvektors $\hat{\mathbf{e}}_{i}^{t}$ über der Wellenlängenachse. In dieser Darstellungsweise können Strukturen oder starke Veränderungen der $x$-Residuen festgestellt werden.

\section{Cook-Einfluss}

Wie bereits des öfteren erwähnt, ist es von großer Bedeutung, ob ein Ausreißer einen starken Einfluss auf ein Modell hat und somit bei der Modellierung nicht berücksichtigt werden sollte, oder ob ein Ausreißer die Modellbildung nicht gefährdet, sondern sogar den Informationsgehalt und die Robustheit eines Modells erhöht. Das in der Chemometrie am häufigsten verwendete Maß hierfür ist das Cooksche Einflussmaß [69]. 
Es berücksichtigt sowohl den Einfluss der $y$-Residuen $\hat{f}_{i}$ als auch den Hebelwert $h_{i}$.

$$
D_{i}=\frac{1}{K+1}\left(\frac{\hat{f}_{i}}{\hat{\sigma} \sqrt{1-h_{i}}}\right)^{2}\left(\frac{h_{i}}{1-h_{i}}\right)
$$

Ein Ausreißer muss demnach einen hohen Hebelwert und hohe $y$-Residuen aufweisen, um einen starken Einfluss auf die Modellbildung zu haben. Ein hoher Wert für $D_{i}$ ist i. d. R. immer ein Hinweis, dass ein solcher Ausreißer aus dem Kalibrierdatensatz entfernt werden sollte. Das Cooksche Einflussmaß wurde urspünglich für die MLR [60] definiert, lässt sich jedoch auch auf PLS und PCR- Modelle anwenden [24].

\subsection{Datenvorbehandlung und -verarbeitung}

Die Güte der Spektren in der NIR-Reflexionsspektroskopie ist sehr stark von den Bedingungen der Messanordnung abhängig. Dabei gibt es nicht nur Einflüsse durch den optischen Aufbau oder den optischen Weg, wie z. B. den Abstand zwischen Messkopf und Probe (Kapitel 5.1.3), sondern auch diverse Einflüsse, die nicht sysmteminhärent sind. So kann z. B. Fremdlicht Anteile von NIR-Licht enthalten und somit zu Signalüberhöhungen auf dem Detektor führen. Aber auch probeninhärente Quereinflüsse wie z. B. eine Veränderung der Probenmatrix durch Korngrößenveränderungen (Kapitel 5.1.1), die Verfärbung der Probe bei einem Röstvorgang (Kapitel 5.1.2) oder eine Veränderung der Probentemperatur (Kapitel 6) können einen starken Einfluss auf die Güte der Spektren und somit die Zuverlässigkeit der Vorhersage eines Materialfeuchtewerts durch Anwendung eines multivariaten Kalibriermodells haben. Diese Einflüsse können mit Hilfe der Anwendung von Datenvorbehandlungsmethoden aus den Spektren mathematisch entfernt werden. Es bleibt jedoch zu beachten, dass in bestimmten Fällen die im Folgenden erläuterten Datenvorbehandlungsmethoden durchaus auch Informationen aus den Spektren entfernen können, die für eine zuverlässige Auswertung notwendig sind.

\subsubsection{Korrektur konstanten Basislinienversatzes}

Die Datenvorbehandlungsmethode zur Korrektur eines konstanten Basislinienversatzes wird auch als ,linear offset correction“ bezeichnet. Das sogenannte „Detrending“ bewirkt die Entfernung von Basislinienversätzen, indem eine Verschiebung des Spektrums in $y$-Richtung durch Abziehen einer horizontalen Linie vom Spektrum entfernt wird. Generell entfernt das Detrending alle rein additiven Einflüsse im Spektrum, sofern sie über den gesamten Spektralbereich von konstanter Natur sind. Das Detrending hat aber auch die Möglichkeit, durch Veränderung der Parameter, Effekte höherer Ordnung wie z. B. das Verkippen eines Spektrums durch Abziehen einer linearen Funktion oder die quadratische Verzerrung, die z. B. von Bräunungseffekten (siehe Kapitel 5.1.2) hervorgerufen werden können, durch Abziehen einer Funktion 2. Ordnung zu entfernen. Meist sind hier aber andere Vorbehandlungsmethoden erfolgreicher und zielführender (siehe Kapitel 4.2.3 und 4.2.4). Die Auswirkung der 


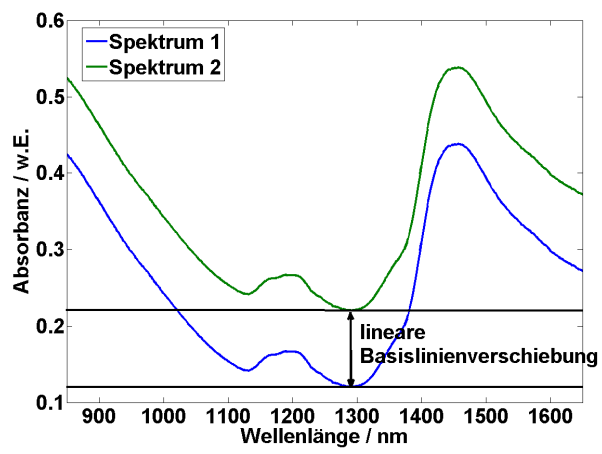

(a) konstante Basislinienverschiebung

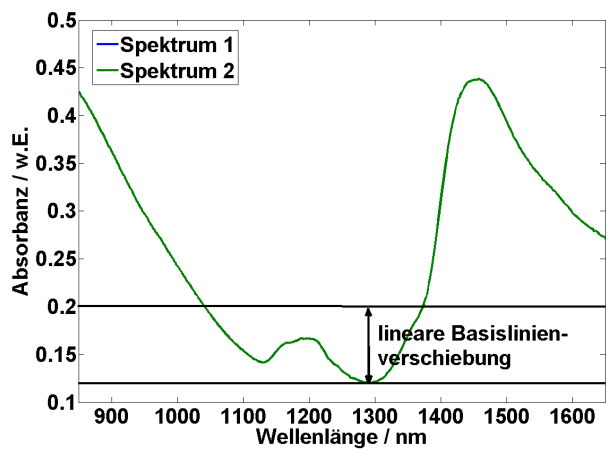

(b) nach Korrektur der konstanten Basislinienverschiebung

Abbildung 4.10: Basislinienkorrektur

linearen Basislinienkorrektur wird in Abbildung 4.10 gezeigt. Mit dieser Datenvorbehandlung können beispielweise folgende Einflüsse im Spektrum enfernt werden:

- Änderungen im Abstand von Probe und Messkopf

- Alterung des Spektrometersystems und der Lichtquelle

\subsubsection{Min-Max-Normierung}

Die Min-Max-Normierung wird auch ,normalization“ genannt und wird zum Vergleich von Spektren angewendet, die z. B. bei unterschiedlichen Pfadlängen bei Küvettenmessungen in Transmission aufgenommen wurden. Bei dieser Normierungsart wird das absolute Minimum des jeweils zu normierenden Spektrums gleich Null gesetzt. Das jeweilige Maximum, auf das normiert werden soll, wird wiederum auf eine Absorptionseinheit gesetzt. Somit können die Spektren leichter auf mögliche multiplikative Einflüsse untersucht werden. Gleichzeitig sind additive Effekte, wie z. B. ein konstanter Basislinienversatz, entfernt. Die Art der Wirkung der Min-MaxNormierung auf zwei voneinander unterschiedliche Spektren zeigt Abbildung 4.11. In Abbildung 4.11(b) lässt sich durch Anwendung dieser Normierung sehr gut der Effekt einer unterschiedlichen Materialfeuchte im Spektrum erkennen, der sich durch Einklappen der auslaufenden Wasserbande im Bereich von 1450-1600 nm äußert. Das Spektrum, dessen Absorbanzverlauf zur Unterscheidung in blau dargestellt wurde, ist von einer Probe mit höherem Materialfeuchtegehalt als die Probe, an der das grün dargestellte Spektrum aufgenommen wurde. Aufgrund dieser Normierung geht allerdings die visuelle Information des Anwachsens der Wasserbande durch Erhöhung der Materialfeuchte verloren. Daher ist diese Datenvorbehandlung auch für die Modellbildung mit solchen Spektren nur bedingt geeignet. 


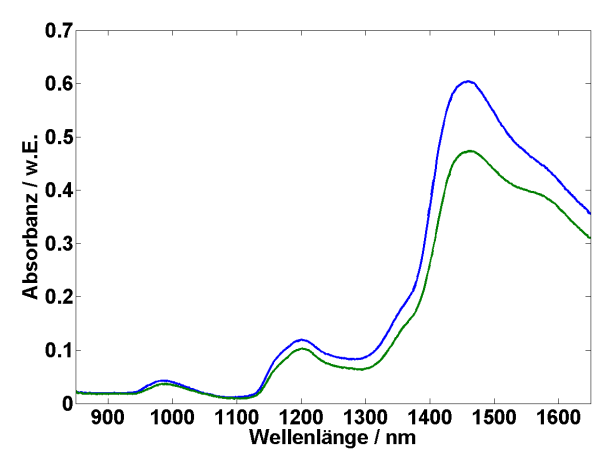

(a) nichtnormierte Spektren

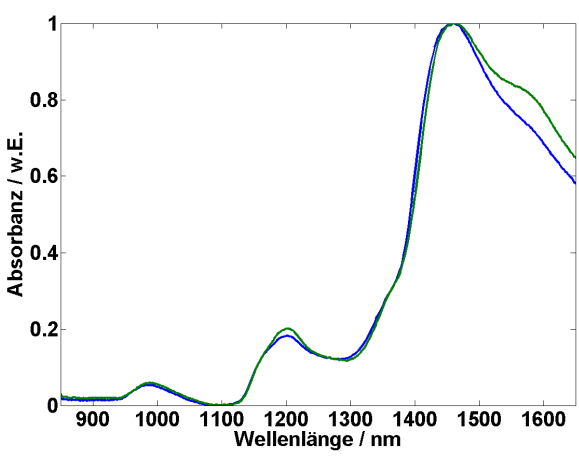

(b) Min-Max-normierte Spektren

Abbildung 4.11: Min-Max-Normierung

\subsubsection{Multiplikative Streukorrektur (MSC)}

Die multiplikative Streukorrektur wird auch als ,multiplicative scatter correction“ bezeichnet und mit MSC abgekürzt. Die Bezeichnung dieser Korrekturmethode lässt bereits vermuten, dass sie Entfernung großer Streuunterschiede z. B. innerhalb einer Produktgruppe verwendet wird. Anhand der Spektren lässt sich eine wellenlängenabhängige Streuung des eingstrahlten Lichts visuell nicht oder nur sehr schwer von der chemisch bedingten Absorption durch z. B. das Wasser in der Probe unterscheiden. Die Streuung bedingt durch die Probenmatrix, also z. B. hervorgerufen durch die Korngröße, könnte anhand des Spektrums einer definierten und charakterisierten Referenzprobe aus dem Datensatz entfernt werden. Der Aufwand hierfür würde aber den normalen Aufwand für die Erstellung einer Kalibrierung bei weitem übersteigen. Die multiplikative Streukorrektur zeigt hier ihren Vorteil. Sie kann die Korrektur auf Basis des vorhanden Datensatzes vornehmen und braucht kein Referenzspektrum. Zur Entfernung der probenbedingten Streuung, wird zunächst das Mittelwertspektrum $\bar{x}^{\text {original }}$ aller Spektren aus dem zu korrigierenden Datensatz bestimmt. Das MSC-korrigierte Spektrum $x_{i}^{\mathrm{MSC}}$ wird dann erhalten, indem dieses Mittelwertspektrum jeweils von dem zu korrigierenden Spektrum $x_{i}^{\text {original }}$ abgezogen wird. Dieses Differenzspektrum wird anschließend auf die Standardabweichung des Spektrums $x_{i}^{\text {original }}$ bezogen auf das Mittelwertspektrum $\bar{x}^{\text {original }}$ normiert. Mathematisch berechnet sich das korrigierte Spektrum mit:

$$
x_{i}^{M S C}=\frac{x_{i}^{\text {original }}-\bar{x}^{\text {original }}}{\hat{x}_{i}}
$$

Diese Methode entfernt sowohl additive als auch multiplikative Effekte aus dem Spektrum. Der Nachteil dieser Korrekturmethode ist, dass ein kompletter Datensatz zur Korrektur benötigt wird. Angewendet wird diese Methode bei Materialien, die untereinander große Streuunterschiede zeigen, z. B. Weizenschrot oder Fleisch [72]. 


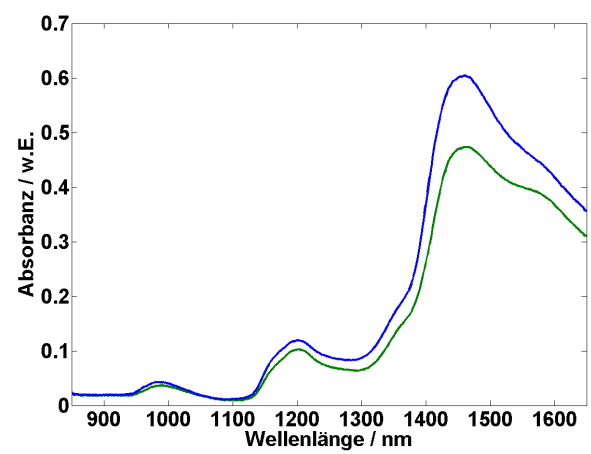

(a) nichtkorrigierte Spektren

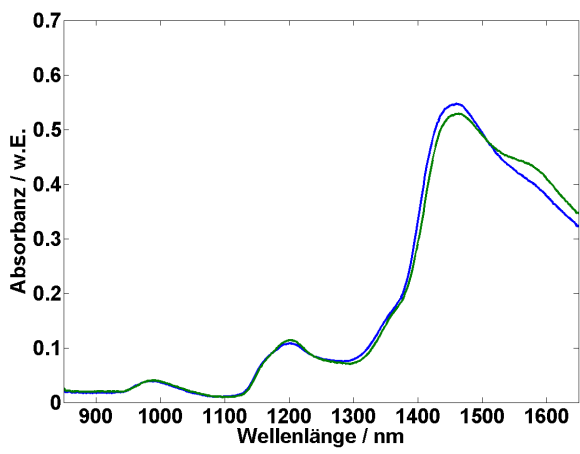

(b) Spektren nach MSC-Korrektur

Abbildung 4.12: Anwendung der multiplikativen Streukorrektur MSC, Darstellung an ausgewählten Spektren

Die Anwendung der multiplikativen Streukorrektur auf Mehlspektren zeigt Abbildung 4.12. Zur Übersichtlichkeit wurden jedoch nur zwei ausgewählte Spektren und nicht der gesamte Datensatz, wie er für diese Methode benötigt wurde, dargestellt. Die Abbildung 4.12(b) zeigt, dass die Anwendung dieser Methode die Verkippung des in blau dargestellten Spektrums gegenüber dem in grün dargestellten Spektrum entfernt hat. Gut zu erkennen ist dieser Effekt, wenn die Flanke der Bande im Bereich von ca. $1300 \mathrm{~nm}$ bis ca. $1400 \mathrm{~nm}$ betrachtet wird, die durch die Anwendung der multiplikativen Streukorrektur fast zur Deckung kommen, was aufgrund der wasserunabhängigkeit dieses Bereichs auch zu erwarten ist. Eine Alternative zu dieser Methode ist die „Standard Normal Variate Methode“, die lediglich das Einzelspektrum mit sich selbst korrigiert. Eine Erläuterung dieses Ansatzes folgt im nächsten Abschnitt.

\subsubsection{Standard Normal Variate (SNV)}

Die Standard Normal Variate Methode (Abkürzung SNV) entfernt sowohl multiplikative als auch additive Effekte im Spektrum. Sie ist der multiplikativen Streukorrektur (MSC) (siehe 4.2.3) sehr ähnlich. Allerdings braucht sie im Gegensatz zur multiplikativen Streukorrektur keinen Spektrensatz, da die Standard Normal Variate Methode das Spektrum auf den Mittelwert der spektralen Stützstellen des jeweiligen Spektrums normiert. Bei der SNV wird jede $i$-te Stützstelle des Spektrums $x$ berechnet mit:

$$
x_{i}^{\mathrm{SNV}}=\frac{x_{i}^{\text {original }}-\bar{x}_{i}^{\text {original }}}{\hat{x}_{i}},
$$

wobei $\bar{x}_{i}^{\text {original }}$ der Mittelwert aller Stützstellen $x_{i}$ des Spektrums und $\hat{x}_{i}$ die Standardabweichung der $i$-ten Stützstelle gegenüber dem Mittelwert aller Stützstellen 


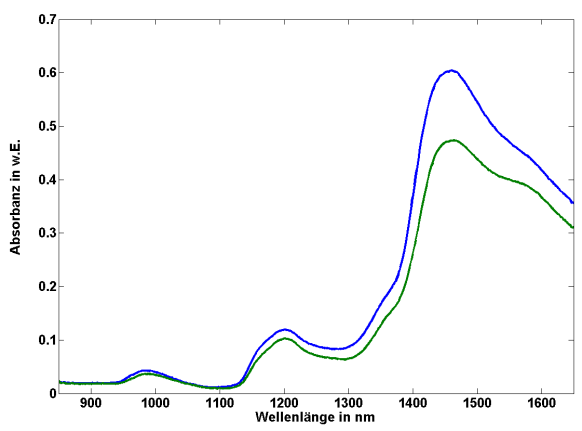

(a) unvorbehandelte Spektren

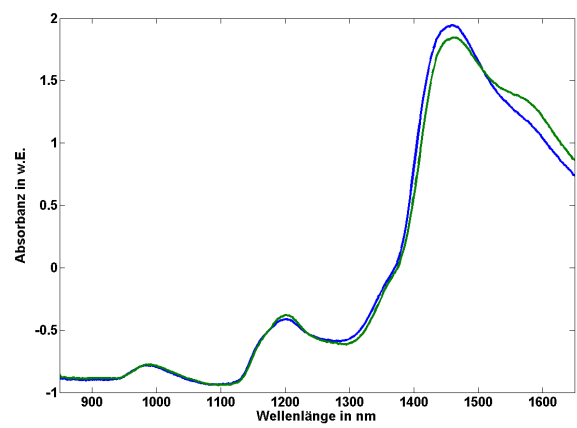

(b) Spektrum nach Anwendung der Standard Normal Variate

Abbildung 4.13: Anwendung der Standard Normal Variate Korrektur SNV

$x_{i}$ des Spektrums ist. Die Awendung der Standard Normal Variate Methode zeigt Abbildung 4.13. Es ist sehr gut zu erkennen, dass die beiden Spektren durch Anwendung dieser Methode sehr gut zur Deckung kommen und sich hauptsächlich nur noch in der Varianz der Wasserbande im Bereich zwischen 1400-1650 nm unterscheiden. Andere Varianzen sind demnach fast vollständig aus den Spektren eleminiert worden.

\subsubsection{Savitzky-Golay-Glättung und Ableitung}

Der Datenvorbehandlungsalgorithmus nach Savitzky und Golay [73] wird in der NIRSpektroskopie standardmäßig zur Glättung von verrauschten Spektren verwendet. Je nach Wahl der Savitzky-Golay-Koeffizienten kann dieser Algorithmus aber auch in einem Schritt eine Glättung und eine erste Ableitung oder eine Glättung kombiniert mit einer Ableitung zweiter Ordnung der spektralen Daten durchfüh-

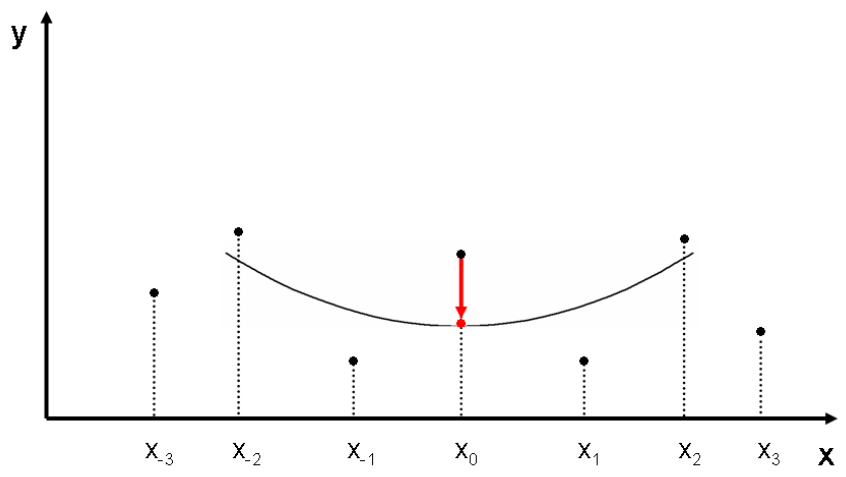

Abbildung 4.14: Funktion der Savitzky-Golay-Glättung

ren. Die Abbildung 4.14 veranschaulicht die Funktionsweise dieses Algorithmus. Sie lässt erkennen, dass die Savitzky-Golay-Datenvorbehandlung der gleitenden Mittelwertbildung sehr ähnlich ist. Beschrieben wird dieser Algorithmus durch die folgende 


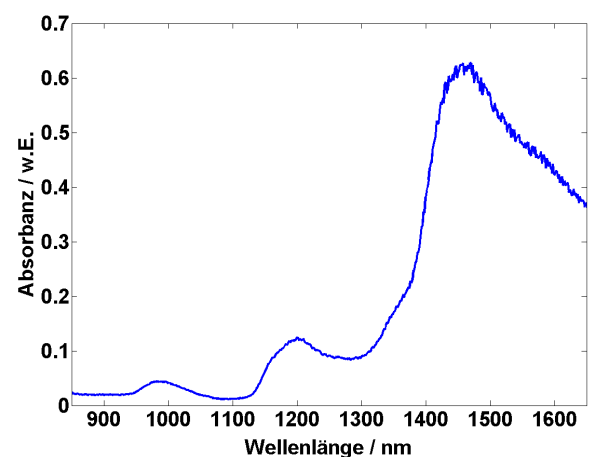

(a) verrauschtes Spektrum

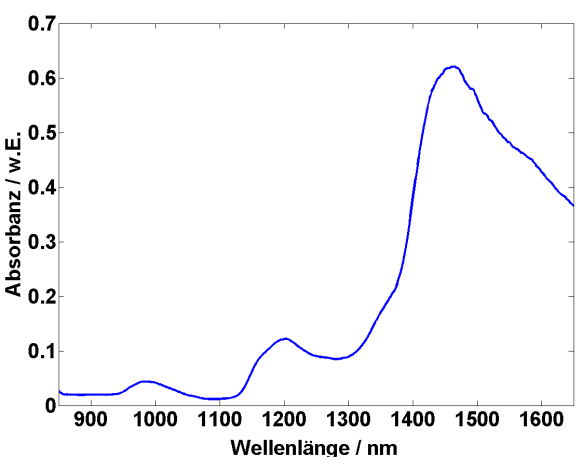

(b) Spektrum nach Glättung durch SavitzkyGolay-Filter

Abbildung 4.15: Anwendung des Savitzky-Golay-Filters

Gleichung:

$$
y_{t}=\frac{1}{h} \sum_{i=-\frac{n-1}{2}}^{\frac{n-1}{2}} a_{i} x_{0+i},
$$

$a_{i}$ sind die Savitzky-Golay-Koeffizienten. $h$ ist ein Normierungsfaktor und wird ebenso wie die Koeffizienten $a_{i}$ aus der Tabelle in der Arbeit von Savitzky und Golay [73] entnommen. $n$ ist die Anzahl an verwendeten Stützstellen für das Glättungsfenster, das wie bei der gleitenden Mittelwertbildung in Wellenlängenrichtung über die Spektren läuft. Die Vorteile des Savitzky-Golay-Algorithmus sind:

- Entfernung des spektralen Rauschens

- Auswertung der Maxima durch höhere Ableitung und gleichzeitige Glättung

- Verhalten des Filters wird durch entsprechende Wahl der Filterkoeffizienten gewählt

Im Gegensatz zur gleitenden Mittelwertberechnung verwendet der Savitzky-GolayFilter ein Polynom zur lokalen Anpassung der Daten. Aufgrund seiner Eigenschaften kann dieser Filter nur bedingt für sehr scharfe Strukturen verwendet werden, da diese möglicherweise als Rauschen interpretiert werden und somit aus den Daten entfernt oder zumindest sehr stark reduziert werden. Abhängig ist dieser Effekt von der Wahl der Filterbreite und dem verwendeten Polynom zur Datenanpassung. Wird dieser Filter zur Ableitung benutzt, müssen auch die Eigenschaften durch die jeweilige Ableitungsart mit in Betracht gezogen werden. So bewirkt eine Ableitung erster Ordnung, dass Signale mit steilem Anstieg mehr an Bedeutung gewinnen als flachere Strukturen. Daher eignet sie sich genauso wie die Glättung besser zur Auswertung breiter Banden und Strukturen, wie sie im NIR fast ausschließlich auftreten. Die 
zweite Ableitung ist vergleichbar mit der ersten Ableitung. Der Vorteil der zweiten Ableitung liegt in der Aufwertung von flacheren Strukturen als bei der ersten Ableitung. Würde diese Methode neben der Ableitung nicht auch gleichzeitig glätten, würde die zweite Ableitung sogar zu einer Aufwertung des Rauschens führen und wäre ohne Glättung nur sehr bedingt einsetzbar. Ein Anwendungsbeispiel der Savitzky-Golay-Glättung zeigt Abbildung 4.15.

\subsection{Entwicklung chemometrischer Methoden}

Die Entwicklung chemometrischer Methoden beruht auf der Erfahrung und dem Wissen des Chemometrikers, der sie entwickelt. Es lässt sich lediglich an Daumenregeln abschätzen, wie eine Kalibrierung erstellt werden muss. Der Chemometriker sieht anhand der einzelnen Darstellungen und Kennwerte seines Modells, ob es robust gegen zu erwartende Einflüsse ist. Erst die Erfahrung und der häufige Umgang mit der Chemometrie erlaubt das Erkennen, in welche Richtung eine Kalibrierung weiterentwickelt werden muss. Ein zunächst als Ausreißer angesehener Datensatz wird sicherlich einen signifikanten Einfluss auf die Modellbildung haben, so lange das Modell nicht bereits zu stark populiert ist. Erst der Chemometriker selbst kann anhand seines Wissens über die Gewinnung und die Bedingungen des Datensatzes, wie z. B. das Erntejahr, die Herkunftsregion oder auch die Sortenzugehörigkeit sicher sagen, ob ein Ausreißer zur Verbesserung der Robustheit eines Modells verwendet werden sollte und möglicherweise noch weitere Proben mit diesen Varianzbeiträgen in die Modellbildung mit einbezogen werden sollten, oder ob ein Datensatz komplett aus einem Modell herausgelassen werden sollte. Es gibt jedoch auch Regeln, die allgemeingültig schriftlich fixiert werden können. So ist das Design eines Kalibrierdatensatzes in einer Norm festgehalten.

\subsubsection{Design eines Kalibrierdatensatzes}

Die Erstellung des Designs eines Kalibrierdatensatzes zur quantitativen multivariaten Auswertung in der Infrarotspektroskopie ist in der ASTM-Norm E1655 [40] festgelegt. Diese Norm beschreibt u. a. in Abhängigkeit von der Probenkomplexität, welche Anzahl an Proben benötigt wird, um eine verlässliche Aussage zu erhalten. Ein repräsentativer Kalibrierprobensatz setzt sich zusammen aus Proben,

- die möglichst alle zu erwartenden chemischen Komponenten beinhalten

- die eine größere Varianz in ihrer chemischen Zusammensetzung zeigen, als die mit dem Modell zu untersuchenden Proben

- deren chemische Komponentenkonzentrationen gleichmäßig über den gesamten Varianzbereich verteilt sind 
- die in derart genügend großer Anzahl vorhanden sind, dass der Zusammenhang zwischen der spektralen Information und den zu modellierenden chemischen Konzentrationen oder Eigenschaften statistisch bestimmt ist

Sollte ein bestimmter Varianzbereich von besonders großem Interesse sein, so kann das Modell an diesen Stellen durch zusätzliche Proben verbessert werden. Es kann aber auch sinnvoll sein, diese speziellen Bereiche aus dem gesamten Kalibrierraum in sogenannte „Unterkalibrierräume“ aufzuteilen.

\subsection{Piecewise Direct Standardization (PDS)}

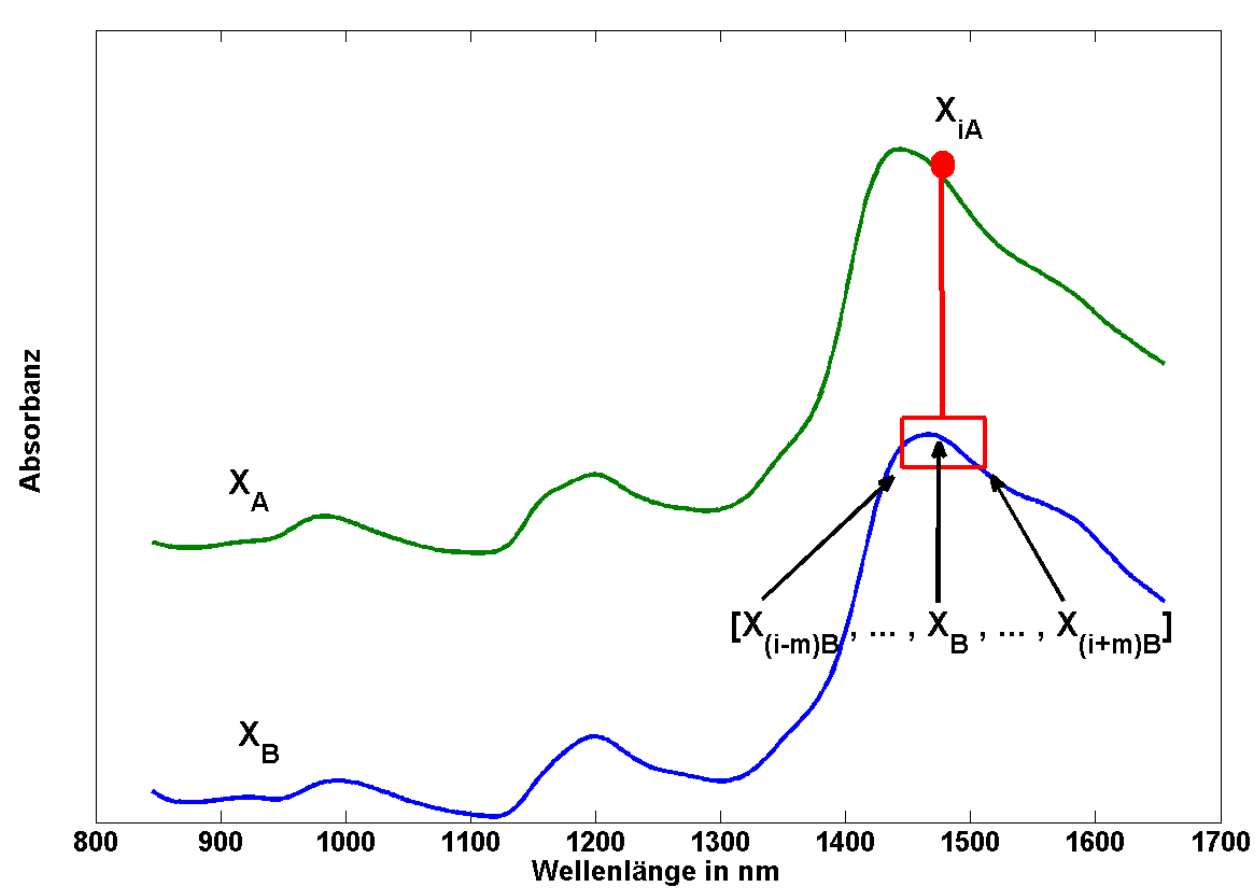

Abbildung 4.16: Funktionsweise der Piecewise Direct Standardization, die beiden Spektren wurden für eine bessere Übersicht gegeneinander verschoben

Der Ansatz zur Korrektur des Temperatureinflusses, wie er in Kapitel 7 verwendet wird, beruht auf einem Algorithmus zum Transfer von Spektrensätzen oder vollständigen Kalibrierungen zwischen zwei Spektrometern. Es gibt einige Gründe, warum eine Kalibrierung, die mit einem Gerät A erstellt wurde, nicht ohne weiteres Spektren eines anderen Gerätes B vorhersagen kann. So können sogar zwischen zwei baugleichen Geräten aus der selben Serie bereits so große bauartbedingte Abweichungen durch Unterschiede in der physikalischen Auflösung des Spektrums bestehen, dass 
eine Kalibrierübertragung von einem auf das andere Gerät nicht ohne Korrektur möglich ist. In der NIR-Spektroskopie sind außerdem verschiedene Technologien für die Aufnahme eines Spektrums zu finden, was wiederum eine Varianz zwischen den Geräten A und B bedeutet. So lassen sich Kalibrierungen, die mit einem FTIRSpektrometer erstellt wurden, nicht direkt auf Spektren, die mit einem Diodenzeilenspektrometer aufgenommen wurden, anwenden (die Erläuterung der einzelnen Technologien ist in [74] zu finden). Aber auch der Transfer einer Kalibrierung von einem Offlinelaborgerät auf ein Prozessmessgerät, das online misst, von einem Gerät mit Freistrahloptik auf ein Gerät, das die Spektren mittels einer Faseroptik aufnimmt, oder ein Unterschied aufgrund der Alterung oder Neujustage eines Gerätes ruft i. d. R. das Bedürfnis nach dem Transfer der Kalibrierung auf das neue Gerät B hervor. In der Literatur sind eine Vielzahl an Methoden zum Transfer einer Kalibrierung zu finden [24, 75, 76, 77]. Zwei dieser Methoden werden als Standardmethoden in den Kapiteln 6.2 und 6.3 untersucht. Die dritte Methode, die Piecewise Direct Standardization [78, 79, 80, 81], wird in Kapitel 7 als Ansatz zur Korrektur des Temperatureinflusses in den nahinfraroten Spektren untersucht. Die Grundlage der Piecewise Direct Standardization ist der Ansatz der „Direct Standardization (DS)“ [24], die einen multivariaten und einen univariaten Ansatz zum Spektrentransfer ermöglicht. Der multivariate Ansatz der „Direct Standardization“ verwendet das gesamte Spektrum zur Berechnung der Transferfunktion, so dass lokale Varianzen in den Spektren nicht hinreichend genug modelliert werden könnten. Der univariate Ansatz wiederum berechnet die Transferfunktion, indem er jede Stützstelle des Spektrums einzeln modelliert, was zwar für einen Unterschied in der Intensität zweier Transferspektren gute Ergebnisse liefern wird, aber Verschiebungen in der Wellenlängenachse nicht berücksichtigen kann.

Die Piecewise Direct Standardization hingegen vereint durch ihren Ansatz die Vorteile der multivariaten und der univariaten Direct Standardization, indem sie jede Stützstelle $x_{\mathrm{iA}}$ des Spektrums $X_{\mathrm{A}}$ des Gerätes A auf einen Wellenlängenbereich $\left[x_{(\mathrm{i}-\mathrm{m}) \mathrm{B}}, \ldots, x_{\mathrm{iB}}, \ldots, x_{(\mathrm{i}+\mathrm{m}) \mathrm{B}}\right]$ des Spektrums $X_{\mathrm{B}}$ des Gerätes B um die selbe Stützstelle $x_{\mathrm{iB}}$ bezieht (Veranschaulichung siehe Abbildung 4.16). Eine lineare Regression der Stützstelle $x_{\mathrm{iA}}$ auf das Fenster der Piecewise Direct Standardization um die Stützstelle $x_{\mathrm{iB}}$ mit der Fensterweite $m$ ergibt ein Regressionsmodell für jede Stützstelle des Spektrums $X_{\mathrm{A}}$ :

$$
\begin{aligned}
& x_{\mathrm{iA}}=X_{\mathrm{iB}} b_{\mathrm{i}}+e_{\mathrm{i}}, \\
& \text { mit } X_{\mathrm{iB}}=\left[x_{(\mathrm{i}-\mathrm{m}) \mathrm{B}}, \ldots, x_{\mathrm{iB}}, \ldots, x_{(\mathrm{i}+\mathrm{m}) \mathrm{B}}\right] \\
& \text { und } e_{\mathrm{i}}: \text { Residuen der Regression. }
\end{aligned}
$$

Der Vektor $b_{\mathrm{i}}$ ist somit der Regressionsvektor der Stützstelle $x_{\mathrm{iA}}$ auf das Fenster $X_{\mathrm{iB}}$. Es ergeben sich folglich $i$ Regressionsvektoren zum Transfer des Spektrums $X_{\mathrm{B}}$ als wäre es auf dem Ursprungsgerät A gemessen worden. Das transferierte Spektrum wird mit $X_{\mathrm{B}(\mathrm{A})}$ symbolisiert. Zusammengefasst werden die Regressionsvektoren $b_{\mathrm{i}}$ in 


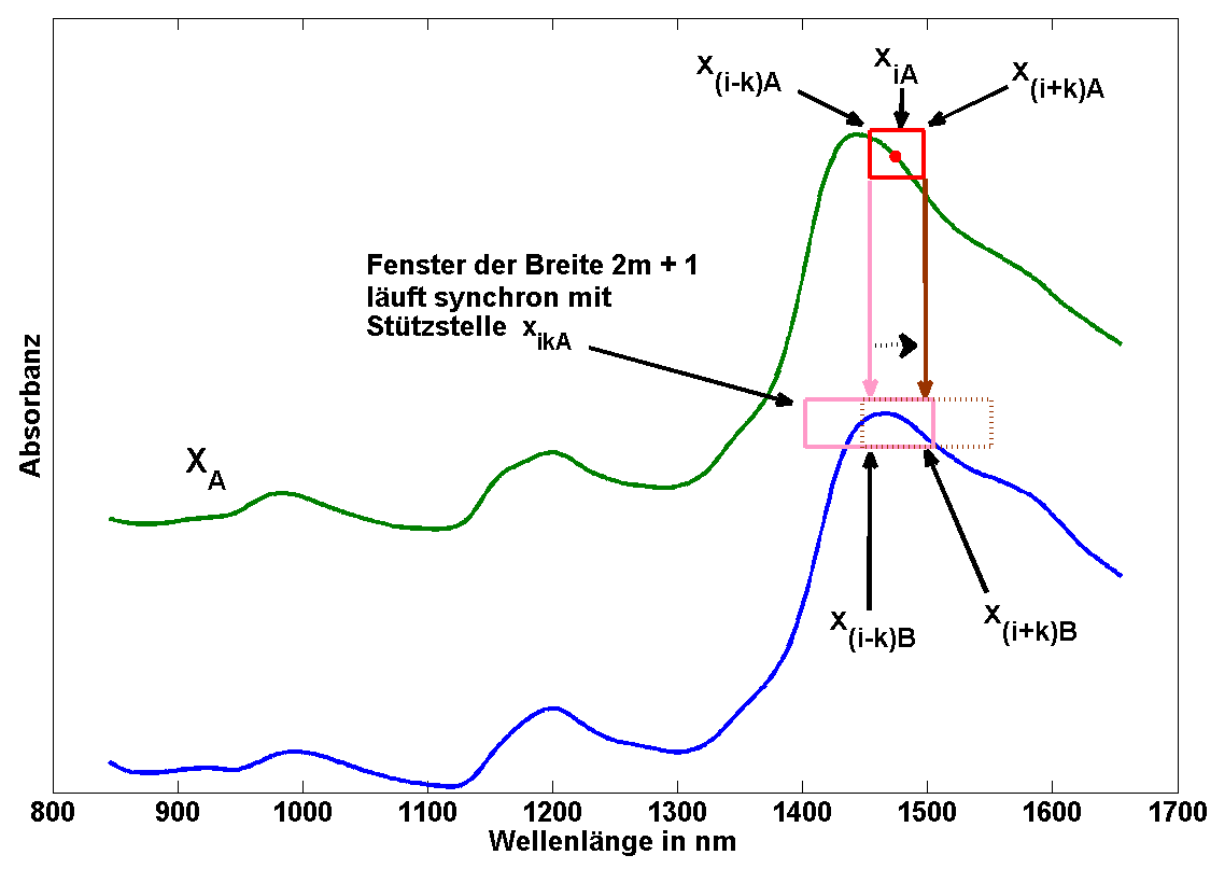

Abbildung 4.17: Funktionsweise der Double Windowed Piecewise Direct Standardization, die beiden Spektren wurden für eine bessere Übersicht gegeneinander verschoben

der Transfermatrix $\hat{B}$, so dass das transferierte Spektrum wie folgt erhalten wird:

$$
X_{\mathrm{B}(\mathrm{A})}^{t}=X_{\mathrm{B}}^{t} \hat{B} \text {. }
$$

Eine Erweiterung der Piecewise Direct Standardization ist die „Double Windowed Piecewise Direct Standardization (DWPDS)“. Sie funktioniert genauso wie die Piecewise Direct Standardization, ergänzt diese aber um ein spektrales Fenster mit der Breite $2 k+1$ um die Stütztelle $x_{\mathrm{iA}}$ im Spektrum $X_{\mathrm{A}}$ des Urgeräts. Diese Transfermethode erstellt somit für jede Stützstelle im Fenster um $x_{\mathrm{iA}}$ einen Regressionsvektor für das zugehörige Fenster im Spektrum $X_{\mathrm{B}}$. Für jede Stützstelle $x_{\text {ikA }}$ des Fensters im Spektrum $X_{\mathrm{A}}$ wird also eine Piecewise Direct Standardization ausgeführt und jeweils ein Regressionsvektor $b_{i k}$ nach Gleichung 4.19 gefunden. Alle Vektoren $b_{i k}$ werden zu einer Matrix $b_{i}$ zusammengefasst, mit deren Hilfe dann das neue Transferspektrum bestimmt werden kann. Die Funktionsweise der Double Windowed Piecewise Direct Standardization ist in Abbildung 4.17 veranschaulicht. Bei der Anwendung der Piecewise Direct Standardization und auch der Double Windowed Piecewise Direct Standardization muss berücksichtigt werden, dass jede ungewollte Varianz, wie z. B. Pixelfehler, wenn sie nicht aus dem Spektrum entfernt wird, mit in die Modellierung der Transfermatrix einfließt. 


\section{Kapitel 5}

\section{Experimentelle Ergebnisse}

Dieses Kapitel stellt die Ergebnisse dar, die im Rahmen dieser Arbeit durch spektroskopische Untersuchungen an verschiedenen Problemstellungen erzielt wurden. Zunächst werden einzelne Quereinflüsse betrachtet, die zu Problemen bei der Erstellung eines chemometrischen Modells führen können. Desweiteren wird der Einfluss einer variierenden Probentemperatur auf das Verhalten chemometrischer Modelle untersucht. Die Materialfeuchtereferenzwerte, die zur Erstellung der Modelle benötigt wurden, sind in Tabelle 3.2 aufgeführt.

\subsection{Quereinflüsse}

Die Vorhersageergebnisse eines chemometrischen Modells sind sehr stark von unterschiedlichen Einflussfaktoren abhängig. So kann eine unterschiedliche Partikelgröße zwischen dem Probenzustand zum Kalibrierzeitpunkt und bei der Vorhersage neuer dem Modell unbekannter Proben bereits einen starken nicht vernachlässig-

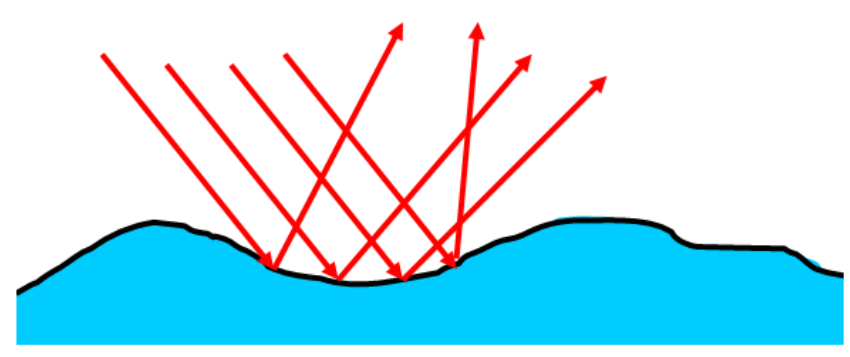

Abbildung 5.1: Diffuse Reflexion an einer Oberfläche baren Einfluss auf die Zuverlässigkeit der Vorhersage des Analyten (hier Materialfeuchte) bewirken (siehe Kapitel 5.1.1). In diesem Fall ist die Messung in diffuser Reflexion ein Grund für diesen Einfluss (siehe Abbildung 5.1). Bei der Messung von Spektren während eines Röstvorgangs kann der Einfluss von Bräunungseffekten Probleme in der Vorhersage der Materialfeuchte bewirken (siehe Kapitel 5.1.2). Dies kann durch Veränderung des Probenspektrums sogar soweit gehen, dass eine Probe nicht mehr zum Kalibrierdatensatz passt und somit ein Ausreißer wird, obwohl sich hauptsächlich die visuellen Eigenschaften, also die Farbe der Probe, verändert hat. In der Reflexionsspektrosko- 


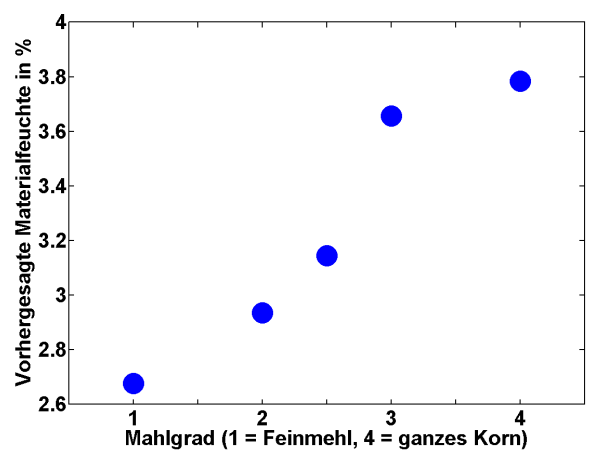

(a) Abhängigkeit der Materialfeuchtevorhersage von der Partikelgröße an Kaffee

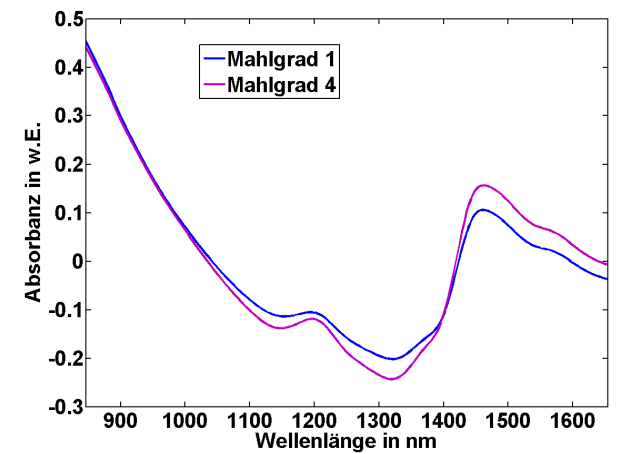

(c) Kaffeepulverspektren geglättet und offsetkorrigiert

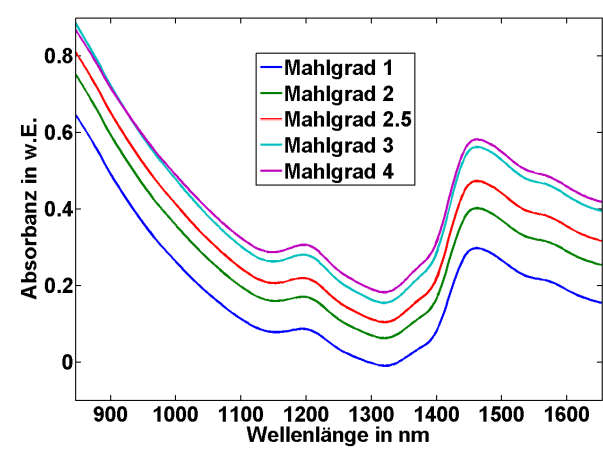

(b) geglättete Spektren von Kaffeepulver

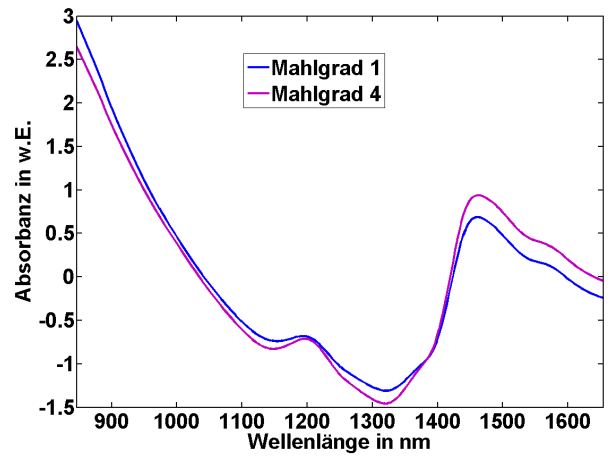

(d) Kaffeepulverspektren geglättet und mit SNV korrigiert

Abbildung 5.2: Einfluss der Korngröße auf die Vorhersage und die Absorbanzspektren von Kaffeepulver anhand verschiedener Mahlgrade

pie ist die Anordnung von Probe und Sensorkopf eine weitere Fehlerquelle, da der Abstand zwischen Probe und Sensorkopf einen entscheidenden Einfluss auf die Größe des Messflecks aber auch auf die vom Messkopf wieder aufgenommene Lichtmenge hat.

\subsubsection{Partikelgröße}

Eine Varianz in der Partikelgröße einer Probe wird sich vermutlich in der Vorhersagequalität eines Modells äußern, da die unterschiedlich großen Partikel ein unterschiedliches Streuverhalten [82] zeigen. Zur genaueren Untersuchung dieses Einflusses auf die Vorhersage, wurde eine Bohnenkaffeeprobe bei unterschiedlichen Mahlgraden mit Hilfe einer Haushaltsmühle ${ }^{1}$ zermahlen und anschließend jeweils ein Spektrum

\footnotetext{
${ }^{1}$ Hersteller: Jupiter, Typ 862
} 
aufgenommen. Der Referenzwert wurde konstant gehalten, indem die gleiche Probe gemahlen, vermessen und dabei Wartezeiten zwischen dem Mahlen und dem Messen geringstmöglich gehalten wurden. Somit ist die zu beobachtende Varianz nur noch vom Mahlgrad abhängig. Die Abbildung 5.2(a) zeigt die Vorhersage eines Kaffeemodells für diese Spektren. Das Modell wurde mit Hilfe diverser unterschiedlicher Kaffeesorten erstellt. Die Spektren des Kalibrierdatensazes wurden vor der Modellberechnung mit den folgenden Datenvorbehandlungen korrigiert:

\section{- Zentrierung}

- standard normal variate SNV

- 1. Ableitung

Es lässt sich leicht erkennen, dass eine starke Abhängigkeit von der Partikelgröße, also dem Mahlgrad, vorhanden ist. Somit ist gezeigt, dass ein chemometrisches Modell sehr stark auf die Varianz der Körnung reagieren kann, sofern diese Varianz nicht mit im Modell berücksichtigt wird. Die Abbildung 5.2(b) zeigt die geglätteten (Savitzky-Golay mit Filterweite 25 Pixel und Polynom 2. Ordnung) Absorbanzspektren von Kaffeepulver mit unterschiedlichen Mahlgraden bei gleicher Materialfeuchte. Es ist gut zu erkennen, dass die Spektren gegeneinander in $y$-Richtung verschoben sind. Die Verschiebung ist um so stärker, je größer der Mahlgrad ist (höherer Mahlgrad bedeutet hier höhere Korngröße). Diese Korrelation könnte auf die unterschiedlichen Streueigenschaften der unterschiedlich großen Partikel zurückzuführen sein. Nach der Kubelka-Munk-Theorie [82] zeigen größere Partikel eine stärkere diffuse Streuung als kleinere Partikel. Demnach würde das Kaffeepulver mit dem höchsten Mahlgrad die stärkste diffuse Streuung bewirken, was wiederum dazu führt, dass über den gesamten Spektralbereich weniger Licht in den Fasermesskopf wieder eingekoppelt wird als bei geringerer Streuung. Dies hat zur Folge, dass die Grundabsorbanz höher wird und somit die Spektren in $y$-Richtung nach oben verschoben werden. Dieser Effekt, auch wenn er hier scheinbar mit der Korngröße korreliert, kann jedoch auch von unterschiedlichen Abständen zwischen Probenoberfläche und Sensorkopf hervorgerufen werden (siehe auch Kapitel 5.1.3). Um den Einfluss der Korngröße aber besser klassifizieren zu können, wurden das Spektrum mit der geringsten Korngröße (Mahlgrad 1) und das Spektrum mit der höchsten Korngröße (Mahlgrad 4) in Abbildung 5.2(c) nach einer Glättung und einer Offsetkorrektur dargestellt. Würden die Spektren keinen Korngrößeneinfluss zeigen, so müssten sie idealerweise direkt aufeinander liegen. Es ist aber leicht zu erkennen, dass neben dem additiven Effekt der Verschiebung in $y$-Richtung auch ein multiplikativer Effekt auf die Form des Spektrums wirkt. Das Spektrum mit Mahlgrad 4 sieht so aus, als wäre es gegenüber dem Spektrum mit Mahlgrad 1 durch eine quadratische Funktion verzerrt worden. Weitere Erkenntnisse, dass der Einfluss der Korngröße sowohl 
additiver als auch multiplikativer Natur ist, gibt die Abbildung 5.2(d). Hier sind die gleichen Spektren wie in der Abbildung 5.2(c) dargestellt. Sie wurden lediglich anstelle mit der reinen Offsetkorrektur mit der standard normal variate (SNV) Methode vorbehandelt. Die SNV korrigiert im Gegensatz zur Offsetkorrektur sowohl additive als auch multiplikative Einflüsse. Mit Hilfe dieser Datenvorbehandlung ist gut zu erkennen, dass der Einfluss der Korngröße definitiv sowohl additiver als auch multiplikativer Natur ist, da die Spektren zwar mittlerweile besser aufeinander liegen, was gut an dem überlappenden Bereich zwischen ca. $1350 \mathrm{~nm}$ und ca. $1450 \mathrm{~nm}$ aber auch am Maximum bei ca. $1200 \mathrm{~nm}$ zu erkennen ist. Es lässt sich aber beobachten, dass die SNV-Datenvorbehandlung nicht alle Effekte des Korngrößeneinflusses vollständig eliminieren kann. Diese Effekte müssen folglich mit in der Modellbildung berücksichtigt werden. Der Vorteil, den die SNV-Datenvorbehandlung in diesem Fall bringt, ist eine Reduzierung des Arbeitsaufwandes, da durch die Reduzierung des Korngrößeneffekts diese Varianz auch im Modell nicht mehr so stark berücksichtigt werden muss. Ein Modell mit dieser Vorbehandlung wird also robuster gegen den Korngrößeneinfluss sein, als ein Modell, das ohne Vorbehandlung erstellt wurde.

\subsubsection{Verfärbungen der Probe}

Die Bräunung einer Probe wird hervorgerufen durch die Maillardreaktion, benannt nach dem Chemiker Louis Camille Maillard, der diese Reaktion 1912 [83, 84, 85] beschrieben hat. Es handelt sich hierbei um eine nicht-enzymatische Veränderung der Zucker und Aminosäuren und ist nicht mit dem Karamellisieren zu verwechseln, wobei beide Reaktionen gemeinsam auftreten können. Die färbenden Substanzen, die bei dieser Reaktion entstehen, werden Melanoide genannt. Die Bräunung, also die Verfärbung der Probe, müsste einen Einfluss auf das spektrale Verhalten einer Probe besonders in der Nähe des sichtbaren Bereichs haben, da der nahinfrarote Spektralbereich direkt an das sichtbare Spektrum anschliesst. Untersucht wurde diese Vermutung, indem Kaffee bei unterschiedlichen Röstgraden spektral vermessen wurde. Die Abbildung 5.3 zeigt exemplarisch an zwei verschiedenen Kaffeeproben mit jeweils einem geringen und einem hohen Röstgrad den spektralen Einfluss dieser Veränderung. Es ist sehr gut zu erkennen, dass im Bereich zwischen 900 und ca. $1200 \mathrm{~nm}$ ein starker Einfluss der Bräunung vorhanden ist, da die Absorption mit abnehmender Wellenlänge steigt. Eine solche Varianz wirft die Vermutung auf, dass eine geeignete Datenvorbehandlung diesen Einfluss minimieren sollte. Experimente mit unterschiedlichen Datenvorbehandlungen haben jedoch gezeigt, dass der Einfluss der Verfärbung kaum zufriedenstellend aus den Spektren entfernt werden kann. Zusätzlich haben diese Untersuchungen gezeigt, dass die Bräunungsinformation in einigen Fällen sogar für die Modellbildung wichtig ist, da sie das Modell gegen Schwankungen in der Probenmatrix robust machen. Im Normalfall führt dieser Einfluss lediglich zu einer bis maximal zwei zusätzlichen Hauptkomponenten, die das Modell somit robuster, aber auch weniger präzise, machen. 


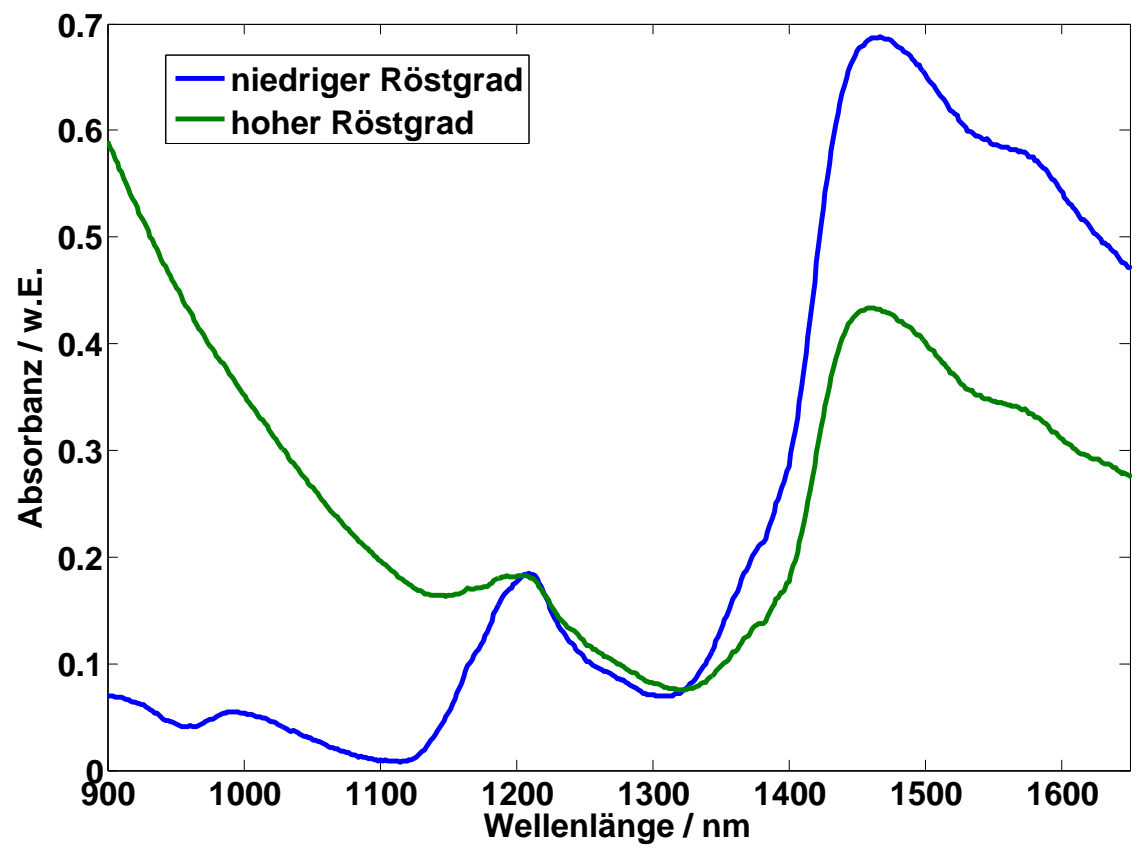

Abbildung 5.3: Einfluss der Probenfärbung / des Röstgrads auf das spektrale Verhalten einer Probe

\subsubsection{Abstand Probe - Sensorkopf}

Die NIR-spektroskopische Messtechnik in Reflexionsanordnung ist i.d. R. ohne zusätzlichen technischen Aufwand, wie z. B. einer Parallelisierungsoptik, sehr stark von der Variation des Abstands zwischen Messkopf und Probenoberfläche abhängig, da die gemessene Intensität bei der Spektrenaufnahme stark variieren kann. Der Grund hierfür ist der von dem Sensor beobachtete Raumwinkel ${ }^{2} \Omega=A / r^{2}$ ( $A$ Kugelfläche des Raumwinkels, $r$ Radius der Kugelfläche). Aufgrund der Apertur des NIR-Messkopfs wird nur ein bestimmter Teil des von der Probe reflektierten Lichts wieder aufgefangen. Die in den Messkopf eingekoppelte Intensität ist abhängig von dem Abstand, da bei größerem Abstand (kleinerem Raumwinkel) aufgrund der Streuung des Lichts an der Probe mehr Intensität verloren gegangen ist bevor das Licht in den Messkopf wieder eintritt als bei kleinerem Abstand und somit größerem Raumwinkel. Zunächst lässt sich nun vermuten, dass das Aufsetzen des Messkopfes auf die Probe die besten Ergebnisse liefern wird, da hier der beobachtete Raumwinkel sehr groß ist und somit das meiste Licht wieder eingekoppelt

\footnotetext{
${ }^{2}$ Als Raumwinkel $\Omega$ bezeichnet man das Verhältnis einer Kugelfläche zum Quadrat ihres Radius [86]. Für die Berechnung des Raumwinkels kann die Kugelfläche hier mit einer Kreisscheibe genähert werden [87]: $\Omega_{\text {Kreisscheibe }}=\pi R^{2} / r^{2}$, R: Radius der Kreisfläche
} 
wird. Hier gibt es jedoch andere Quereinflüsse, die diese Lösung nicht ermöglichen, da eine reale Probe stets auch bei bester Homogenisierung lokal unterschiedliche Feuchtewerte aufzeigen wird, so dass eine Bestimmung der Feuchte in einem möglichst kleinen Probenfleck (Aufsetzen) keine verlässlichen Ergebnisse liefern würde.

Zusätzlich spielen auch unterschiedliche
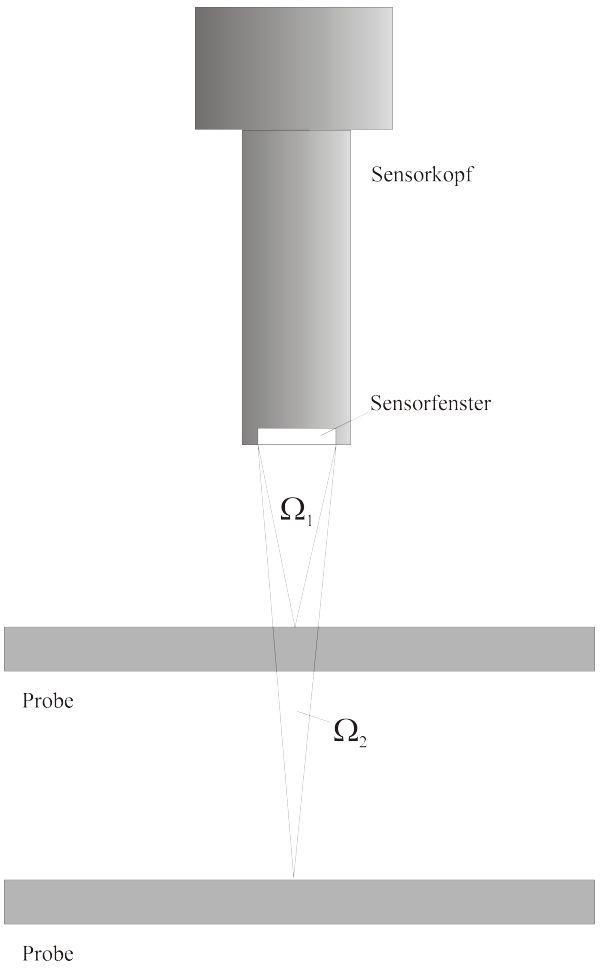
Korngrößen und die Oberflächenstruktur der Probe eine Rolle bei der Streuung des reflektierten Lichtes. Bei größerem Abstand des Messkopfes zur Probe wird auch ein größerer Fleck der Probe ausgeleuchtet, so dass das Ergebnissspektrum über eine größere Fläche gemittelt wird, als wenn der Messkopf aufgesetzt würde. Bei der Mittelung über eine möglichst große Fläche (Fläche der Ausleuchtung) werden folglich die Korngröße, die Oberflächenstruktur und die lokale Feuchteverteilung in der Probe einen viel kleineren Einfluss auf das Messergebniss zeigen. Daher muss für den Arbeitsabstand ein Kompromiss zwischen Ausleuchtungsfläche (Mittelung, möglichst großer Abstand) und aufgenommener Intensität (großes Signal, möglichst kleiner Abstand) gefunden werden [56]. Die Abbildung 5.5 zeigt die Abstands-

Abbildung 5.4: Raumwinkelabhängigkeit abhängigkeit der Vorhersage eines Kalivom Probenabstand briermodells zur Bestimmung der Materialfeuchte von Mehl. Die rote horizontale

Linie zeigt den aufgrund der Referenzanalytik zu erwartenden Materialfeuchtewert. Bereits in dieser Auftragung lässt sich sehr gut erkennen, dass der optimale Arbeitsabstand im Bereich zwischen $150 \mathrm{~mm}$ und $200 \mathrm{~mm}$ liegt. Auch die Darstellung der Residuen der Vorhersage gegenüber dem zu erwartenden Referenzwert zeigt, dass die Abweichung der Vorhersage vom Materialfeuchtereferenzwert in diesem Bereich am geringsten ist. Vorherige Untersuchungen im Rahmen dieser Arbeit haben gezeigt, dass ein Aufbau eines Fasermesskopfes ohne Parallelisierungsoptik eine Abstandstoleranz von lediglich $\pm 3 \mathrm{~mm}$ zeigt. Daher wurde der in dieser Arbeit verwendete Messkopf mit einer Parallelisierungsoptik ausgestattet, so dass sich der optimale Arbeitsabstand im Bereich zwischen $150 \mathrm{~mm}$ und $200 \mathrm{~mm}$ befindet. Aus technischen Gründen wurde der Messabstand zwischen Probe und Sensorkopf für die Untersuchungen dieser Arbeit auf $160 \mathrm{~mm}$ festgelegt, so dass eine Schwankung der Probenhöhe von $\pm 10 \mathrm{~mm}$ kein Problem darstellte. 

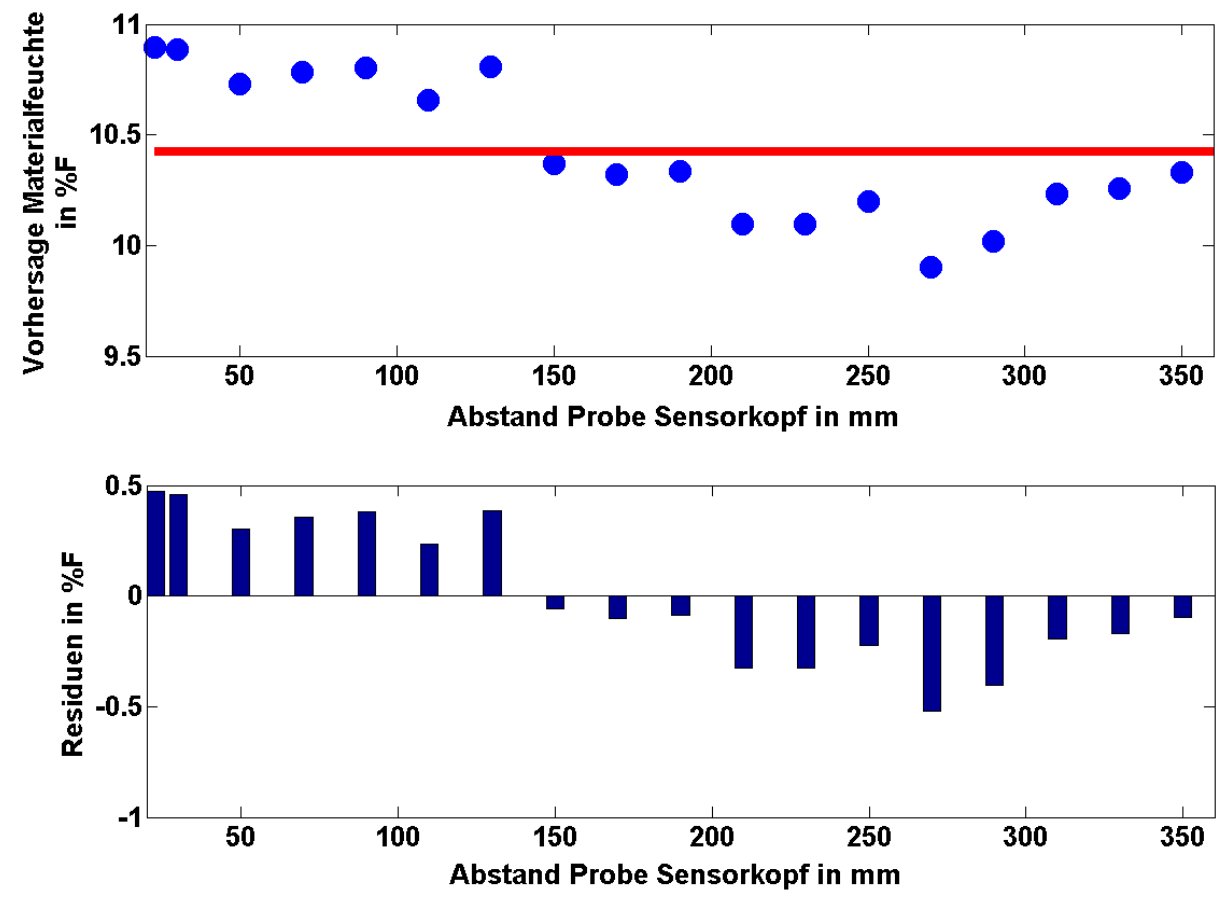

Abbildung 5.5: Abhängigkeit der Materialfeuchtevorhersage vom Abstand zwischen Probe und Sensorkopf mit Residuenplot

\subsection{Einfluss der Probentemperatur auf die Vorhersagen eines Kalibriermodells}

In diesem Kapitel soll der Einfluss der Temperatur auf die Vorhersage eines zu entwickelnden Kalibriermodells untersucht werden. Dazu wurde zunächst ein Modell bei einer Temperatur von $20^{\circ} \mathrm{C}$ entwickelt. Die hierfür verwendeten Proben wurden nach den Regeln zur Erstellung eines Kalibrierdatensatzes (siehe Kapitel 4.3.1 auf Seite 71) ausgewählt. Dieser Datensatz besteht somit aus den Spektren der Mehle mit den Probenbezeichnungen M09, M12, M13, M17, M18, M19, M21 und M45 (nähere Erläuterung siehe Tabelle A.1 auf Seite 115). Zum Vergleich der Vorhersageergebnisse eines für eine Temperatur von $20^{\circ} \mathrm{C}$ erstellten Modells wurden zunächst die statistischen Kennwerte ermittelt. Hierzu wurden die Spektren des für $20^{\circ} \mathrm{C}$ erstellten Modells mit diesem Modell vorhergesagt und die vorhergesagten Feuchte- mit den Referenzfeuchtewerten verglichen. Anschließend wurden die Spektren der Datensätze für eine steigende Temperatur vorhergesagt und wiederum mit den zugehörigen Referenzfeuchtewerten verglichen. Die Spektren wurden mit dem in Kapitel 3.3.2 auf Seite 38 beschriebenen Aufbau aufgenommen. Die Temperatur wurde dabei zusätzlich zu der $20^{\circ} \mathrm{C}$ Stufe auf Stufen von $30^{\circ} \mathrm{C}, 40^{\circ} \mathrm{C}, 50^{\circ} \mathrm{C}, 60^{\circ} \mathrm{C}, 70^{\circ} \mathrm{C}$ und 


\begin{tabular}{rrrr}
\hline $\begin{array}{r}\text { Temperaturstufe } \\
\text { am Thermostat }\end{array}$ & $\begin{array}{r}\text { RMSEP für } \\
\text { Modell bei } \\
\mathbf{2 0} \mathbf{C}^{\circ}\end{array}$ & $\begin{array}{r}\text { Achsenabschnitt } \\
\text { der Regressions- } \\
\text { geraden }\end{array}$ & $\begin{array}{r}\text { Steigung der Re- } \\
\text { gressionsgeraden }\end{array}$ \\
$20^{\circ} \mathrm{C}$ & 0,224 & 0,059 & 0,99 \\
$30^{\circ} \mathrm{C}$ & 0,996 & 0,27 & 1,1 \\
$40^{\circ} \mathrm{C}$ & 2,374 & 0,4 & 1,2 \\
$50^{\circ} \mathrm{C}$ & 4,206 & 0,43 & 1,3 \\
$60^{\circ} \mathrm{C}$ & 6,250 & 0,026 & 1,5 \\
$70^{\circ} \mathrm{C}$ & 9,044 & $-0,42$ & 1,8 \\
$80^{\circ} \mathrm{C}$ & 11,858 & $-0,66$ & 2,1 \\
\hline
\end{tabular}

Tabelle 5.1: Kenngrößen der Vorhersage von Materialfeuchtewerten bei unterschiedlichen Temperaturen, Kalibrierung auf alle Mehle M09, M12, M13, M17, M18, M19, M21 und M45 bei einer Temperatur von $20^{\circ} \mathrm{C}$ mit einem Kreuzvalidierungsfehler $R M S E C V=0,28241$ und Vorhersage derselben Mehle bei den genannten Temperaturstufen

$80^{\circ} \mathrm{C}$ am Thermostat eingestellt. Die Probentemperaturen für die jeweiligen Spektrenmessungen sind in den Tabellen im Kapitel A.2 auf Seite 116 dargestellt. Die Ergebnisse der Vorhersage der Feuchtewerte mit dem für $20^{\circ} \mathrm{C}$ erstellten Modell für die eben erwähnten Temperaturstufen sind in Tabelle 5.1 aufgeführt. Eine Diskussion der hier vorgestellten Ergebnisse folgt in Kapitel 6.1. Zur weiteren systematischen Untersuchung des Einflusses der Temperatur auf die Vorhersage mit einem festen Kalibriermodell bei varierender Temperatur wurde der Datensatz gemäß den Regeln nach Kapitel 4.3.1 in eine Kalibrier- und einen Validierdatensatz aufgeteilt. Dadurch wurden die Mehle M09, M12, M13, M17, M18 und M19 mit den zugehörigen Referenzfeuchtewerten zum Kalibrierdatensatz zusammengefasst. Die Mehle M21 und M45 wurden zusammen mit den entsprechenden Referenzfeuchtewerten jeweils in einem Validierdatensatz vereinigt. Das Kalibriermodell wurde anschließend analog zu dem vorher beschriebenen Vorgehen auf die nun vom Kalibrierdatensatz unabhängigen Spektren angewendet. Durch die Aufteilung der Datensätze waren die beiden Mehle M21 und M45 nicht in dem Datensatz des Kalibriermodells für $20^{\circ} \mathrm{C}$ enhalten. Somit wurde hier gleichzeitig die Robustheit des Modells auf unbekannte Mehlproben geprüft. Die Ergebnisse für diese Auswertungsart zeigt Tabelle 5.2. Beide in diesem Kapitel dargestellten Modelle haben folgende Parameter:

- Datenvorbehandlung ${ }^{3}$

Savitzky-Golay-Glättung Filterweite 25 Pixel und quadratisches Polynom standard normal variate (SNV)

\footnotetext{
${ }^{3}$ Aufzählungsreihenfolge ist Reihenfolge der Anwendung auf die Daten
} 
Mean Centering

- Spektralbereich

$845-1655 \mathrm{~nm}$

- Hauptkomponentenanzahl

5 Faktoren

- Feuchtebereich

$5,48-18,16 \% \mathrm{~F}$

\begin{tabular}{rrrr}
\hline Temperaturstufe & $\begin{array}{r}\text { RMSEP für } \\
\text { Modell bei }\end{array}$ & $\begin{array}{r}\text { Achsenabschnitt } \\
\text { der Regressions- } \\
\text { am Thermostat }\end{array}$ & $\begin{array}{r}\text { Steigung der Re- } \\
\text { gressionsgeraden }\end{array}$ \\
& $\mathbf{2 0}^{\circ} \mathrm{C}$ & $-0,3$ & 1,0 \\
$20^{\circ} \mathrm{C}$ & 0,436 & 0,3 & 1,1 \\
$30^{\circ} \mathrm{C}$ & 1,031 & 0,43 & 1,2 \\
$40^{\circ} \mathrm{C}$ & 2,8 & 0,22 & 1,4 \\
$50^{\circ} \mathrm{C}$ & 4,678 & $-0,012$ & 1,6 \\
$60^{\circ} \mathrm{C}$ & 7,19 & $-0,04$ & 1,9 \\
$70^{\circ} \mathrm{C}$ & 10,307 & $-0,31$ & 2,1 \\
$80^{\circ} \mathrm{C}$ & 13,454 & &
\end{tabular}

Tabelle 5.2: Kenngrößen der Vorhersage von Materialfeuchtewerten bei unterschiedlichen Temperaturen, Kalibrierung auf die Mehle M09, M12, M13, M17, M18 und M19 bei einer Temperatur von $20^{\circ} \mathrm{C}$ mit einem Kreuzvalidierungsfehler $R M S E C V=0,22093$ und Vorhersage der Mehle M21 und M45 bei den genannten Temperaturstufen

Eine detaillierte Betrachtung der Ergebnisse der Untersuchung des Einflusses einer variierenden Probentemperatur auf den Vorhersagefehler RMSEP und damit einhergehend die Steigung der linearen Regression der Vorhersage- und Referenzwerte wird in Kapitel 6.1 dargestellt.

\subsection{Veränderung der Kenngrößen von Kalibriermodellen aufgrund von Veränderungen der Probentemperatur}

Im Kapitel 5.2 wurde der Einfluss einer variierenden Probentemperatur auf die Kenngrößen der Vorhersage eines Kalibriermodells, erstellt bei konstanter Temperatur, untersucht. Dieses Kapitel widmet sich nun der Auswirkung einer variierenden 
Probentemperatur auf die Kenngrößen bei der Modellerstellung und folglich auch auf die Kenngröße der Vorhersage des jeweiligen Modells. Hierzu wurden die Datensätze wie bisher in einen Kalibrier- und einen Validierdatensatz für die jeweilige Temperaturstufe aufgeteilt. Die Mehle M09, M12, M13, M17, M18 und M19 bildeten zusammen mit ihren Referenzfeuchtewerten wiederum die Kalibrierdatensätze. Die Validierdatensätze wurden analog zum bisherigen Vorgehen wiederum aus den Spektren der Mehlsorten M21 und M45 erstellt. Die Ergebnisse dieser Modellbildungen sind in Tabelle 5.3 dargestellt.

\begin{tabular}{rrrr}
\hline $\begin{array}{r}\text { Temperaturstufe } \\
\text { am Thermostat }\end{array}$ & $\begin{array}{r}\text { Hauptkom- } \\
\text { ponentenan- } \\
\text { zahl }\end{array}$ & $\begin{array}{r}\text { Kreuzvalidie- } \\
\text { rungsfehler } \\
\text { RMSECV }\end{array}$ & $\begin{array}{r}\text { Vorhersagefehler } \\
\text { RMSEP für } \\
\text { jeweiliges Modell }\end{array}$ \\
$20^{\circ} \mathrm{C}$ & 5 & 0,221 & 0,436 \\
$30^{\circ} \mathrm{C}$ & 5 & 0,265 & 0,413 \\
$40^{\circ} \mathrm{C}$ & 5 & 0,292 & 0,313 \\
$50^{\circ} \mathrm{C}$ & 5 & 0,348 & 0,508 \\
$60^{\circ} \mathrm{C}$ & 5 & 0,34 & 0,536 \\
$70^{\circ} \mathrm{C}$ & 5 & 0,309 & 0,598 \\
$80^{\circ} \mathrm{C}$ & 5 & 0,399 & 0,643 \\
Modell für alle & & & 0,75499 \\
Temperaturstufen & 7 & 0,47003 & \\
$20^{\circ} \mathrm{C}, 30^{\circ} \mathrm{C}, 40^{\circ} \mathrm{C}$, & & & \\
$50^{\circ} \mathrm{C}, 60^{\circ} \mathrm{C}, 70^{\circ} \mathrm{C}$, & & & \\
$80^{\circ} \mathrm{C}$ & & &
\end{tabular}

Tabelle 5.3: Kenngrößen der Vorhersage von Materialfeuchtewerten bei unterschiedlichen Temperaturen, Kalibrierung auf die Mehle M09, M12, M13, M17, M18 und M19 bei den jeweils erwähnten Temperatureinstellungen des Thermostats (Zusammenhang mit Probentemperatur siehe A.2 auf Seite 116) und Vorhersage der Mehle M21 und M45 bei den genannten Temperaturstufen

Alle in diesem Kapitel dargestellten Modelle haben folgende gemeinsame Parameter:

- Datenvorbehandlung ${ }^{4}$

Savitzky-Golay-Glättung Filterweite 25 Pixel und quadratisches Polynom standard normal variate (SNV)

Mean Centering

\footnotetext{
${ }^{4}$ Aufzählungsreihenfolge ist Reihenfolge der Anwendung auf die Daten
} 
- Spektralbereich

$845-1655 \mathrm{~nm}$

\section{- Feuchtebereich}

$$
5,48-18,16 \% \mathrm{~F}
$$

Um sowohl die Veränderung des Kreuzvalidierungsfehlers $R M S E C V$ als auch des Vorhersagefehlers RMSEP bei den jeweiligen Validierdatensätzen der einzelnen Temperaturmodelle betrachten zu können, wurden diese beiden Kenngrößen in Abbildung 5.6 in Abhängigkeit von der jeweiligen Kalibriertemperatur des Modells dargestellt. Ganz rechts im Bild findet sich in beiden Teildarstellungen der jeweilige Kennwert für ein Modell, das über alle Temperaturen erstellt wurde. Auch diese Modelle wurden jeweils auf einen Validierdatensatz, zusammengesetzt aus den Spektren der Mehle M21 und M45, für alle Temperaturstufen angewendet, wie es in Tabelle 5.3 dargestellt wurde. Sowohl für den Kreuzvalidierungsfehler $R M S E C V$ als auch für den Vorhersagefehler RMSEP ist in erster Näherung ein linearer Zusammenhang mit steigender Temperatur zu beobachten. Dies zeigt auch ein ähnliches Verhalten der Ausgleichsgeraden für die beiden Kenngrößen in dieser Betrachtungsweise (siehe Gleichungen der Regressionsgeraden in der jeweiligen Abbildung). Demnach lässt sich feststellen, dass mit steigender Temperatur beide Kenngrößen linear anwachsen. Zusätzlich lässt sich sogar die Modellbildung über alle Temperaturen in diesen Zusammenhang einordnen. Diese Beobachtung lässt sich bei der Betrachtung der Anzahl an Hauptkomponenten zusätzlich untermauern. Die Hauptkomponentenanzahl zur Beschreibung aller signifikanten Varianzen im Spektrum steigt gegenüber den Modellen für Einzeltemperaturen, wenn alle Spektren unterschiedlicher Temperaturen in ein Modell zusammengefasst werden. Dies ist kein überraschendes Verhalten, da die variierende Temperatur, wie die Beschreibung schon impliziert, eine weitere Varianz ins Spektrum bringt. Somit benötigt ein Kalibriermodell zusätzliche Hauptkomponenten zur Beschreibung dieses Einflusses. Eine Erhöhung der Hauptkomponentenzahl um 2 Hauptkomponenten deutet allerdings bereits auf einen nichtlinearen Einfluss der Temperatur auf die Modellbildung hin, da eine zusätzliche lineare Varianz im Spektrendatensatz mit nur einer weiteren Hauptkomponente zu beschreiben wäre. Weitere Ergebnisse und daraus resultierende Schlussfolgerungen sind in Kapitel 6 zu finden.

\subsection{Zusammenfassung der experimentellen Ergebnisse}

In diesem Kapitel wurden die grunlegenden experimentellen Ergebnisse dieser Arbeit dargestellt und diskutiert. Es wurden verschiedene Quereinflüsse, die eine spektroskopische Messung verfälschen können, näher betrachtet. Dabei zeigte sich, dass der 

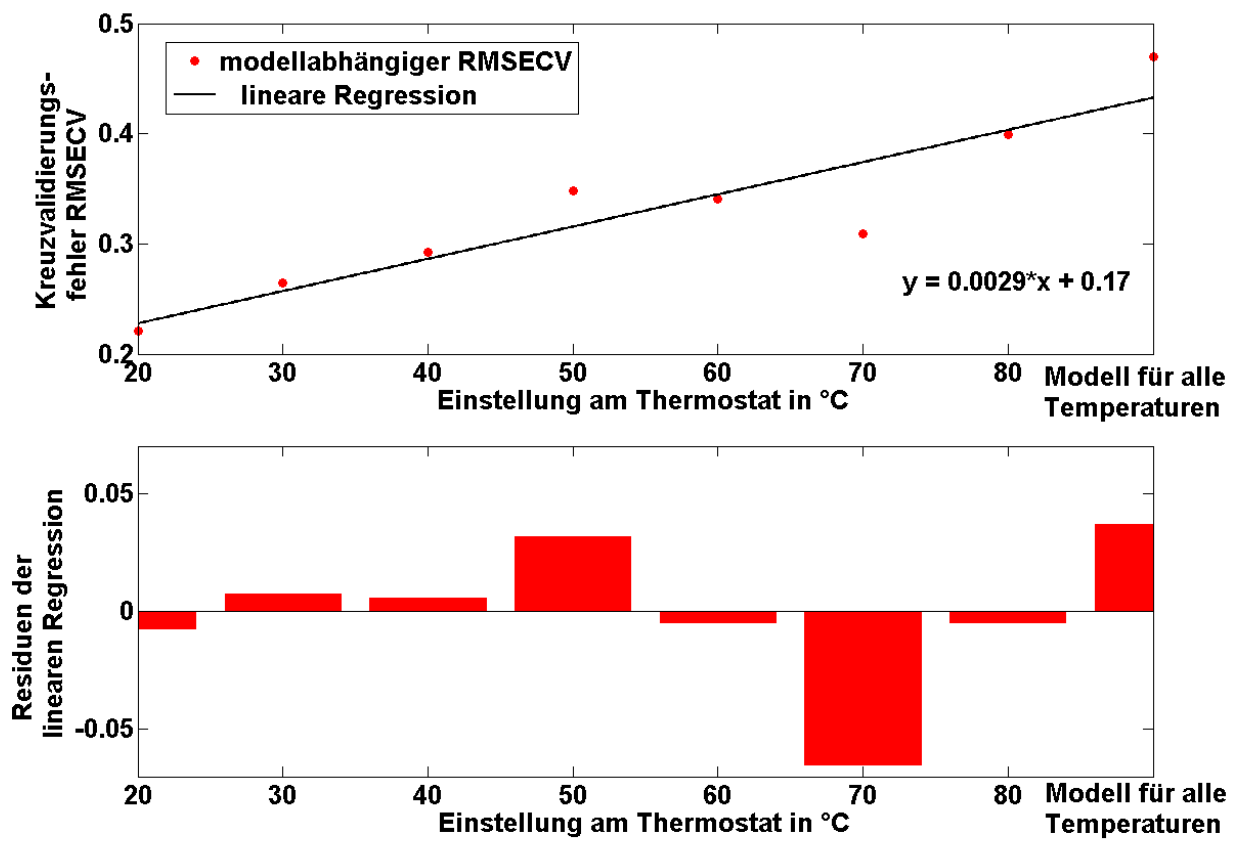

(a) temperaturabhängiger Kreuzvalidierungsfehler $R M S E C V$
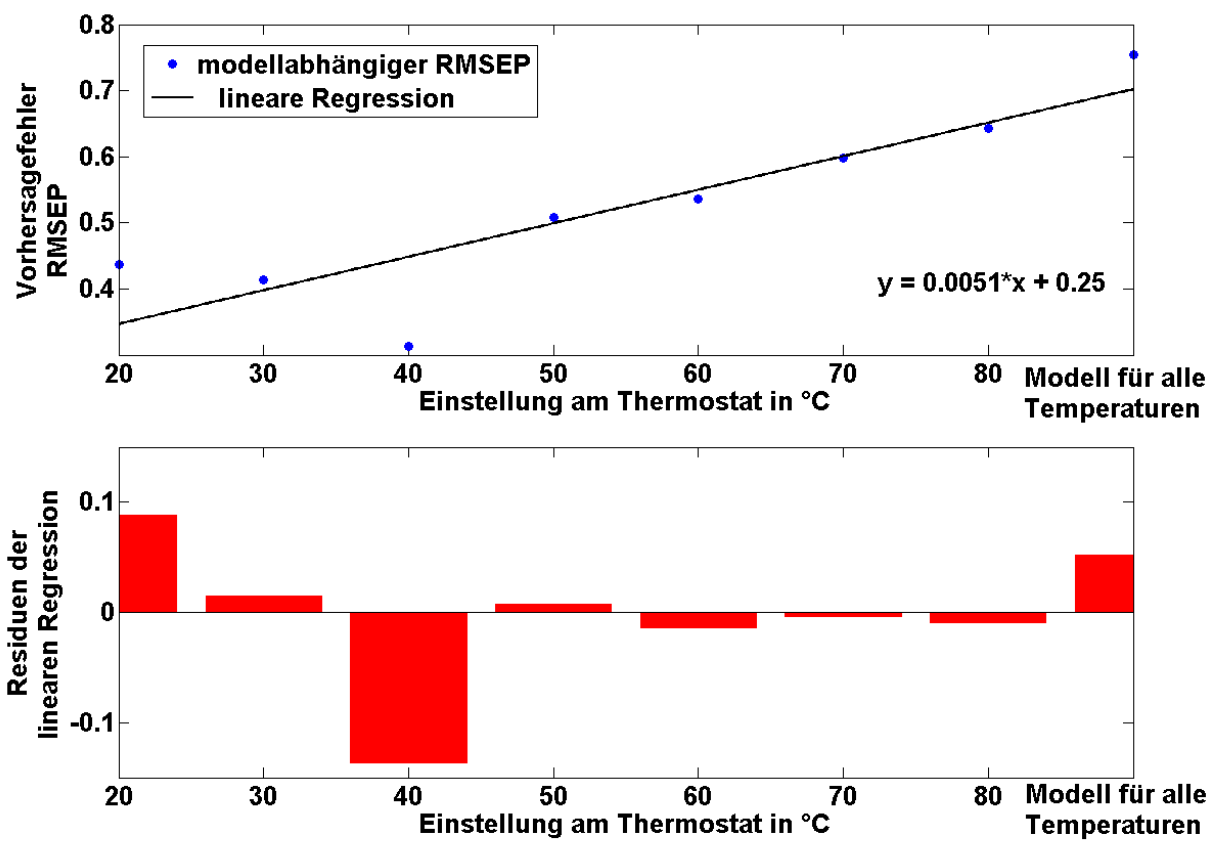

(b) temperaturabhängiger Vorhersagefehler RMSEP

Abbildung 5.6: Kreuzvalidierungsfehler RMSECV und Vorhersagefehler $R M S E P$ verschiedener Modelle für definierte Temperaturstufen analog zu Tabelle 5.3 
Einfluss einer Varianz in der Korngröße bei einer spektroskopischen Messung zu sehr großen Fehlern in der Vorhersage des zu bestimmenden Paramters führen kann (Kapitel 5.1.1). Auch die Verfärbung der Probe (Kapitel 5.1.2) oder eine Änderung des Abstands zwischen Probe und Sensorkopf (Kapitel 5.1.3) sind nicht zu vernachlässigende Einflüsse, die somit bei den weiteren Messungen im Rahmen dieser Arbeit berücksichtigt und mit der Wahl von Mehl als Modellsubstanz und der Einhaltung eines festen Abstands zwischen Sensor und Probe als Einfluss minimiert werden konnten.

Im nächsten Schritt wurde untersucht, welchen Einfluss die Probentemperatur auf die Vorhersage eines Modells hat, indem ein Modell bei einer konstanten Temperatur erstellt wurde und Spektren verschiedener Temperatur vorhergesagt wurden. Mit diesen Betrachtungen konnte gezeigt werden, dass der Vorhersagefehler eines bei fester Temperatur erstellten Modells linear mit der Probentemperatur wächst (Kapitel 5.2 und Diskussion in Kapitel 6.1).

Weiterhin wurde der Einfluss einer variierenden Probentemperatur auf die Modellbildung untersucht. Dazu wurden Modelle auf Basis von Datensätzen bei unterschiedlichen Temperaturen erststellt und die Kenngrößen wie Kreuzvaldierungsfehler, Anzahl der Hauptkomponenten und Vorhersagefehler untersucht (Kapitel 5.3). Es hat sich gezeigt, dass sowohl der Kreuzvalidierungsfehler als auch der Vorhersagefehler linear mit der Probentemperatur wachsen. Die Vereinigung von Spektren unterschiedlicher Temperatur in einem Gesamtdatensatz zeigte eine Erhöhung der Hauptkomponentenanzahl um 2 Hauptkomponenten (siehe Tabelle 5.3 auf Seite 84). Dies ist bereits ein Hinweis auf einen nichtlinearen Einfluss der Temperatur auf die Spektren, da eine lineare Varianz bei der Modellbildung lediglich eine weitere Hauptkomponente zur Folge haben würde. Nichtlineare Einflüsse müssen in dem linearen Ansatz der chemometrischen Methoden meist mit mindestens zwei Hauptkomponenten zusätzlich zu der Hauptkomponentenzahl des nichtbeeinflussten Modells modelliert werden. 



\section{Kapitel 6}

\section{Diskussion}

\subsection{Verhalten der Vorhersage bei variierender Temperatur}

\subsubsection{Zusammenhang Vorhersagefehler und Steigung der linearen Regression}

Dieses Kapitel stellt die Untersuchungen des Verhaltens der Steigung der linearen Regression über den vom Modell vorhergesagten und dem Referenzwert dar. Es zeigt den Zusammenhang dieses Parameters mit dem Vorhersagefehler RMSEP auf. Das für diese Untersuchungen verwendete Modell wurde bereits in Kapitel 5.2 besprochen. Die Abbildung zeigt die Darstellung des Zusammenhangs zwischen diesen beiden Werten. Dabei entspricht jeder RMSEP-Wert dem Vorhersagefehler des Modells aus Kapitel 5.2 für eine Temperaturstufe von $20^{\circ} \mathrm{C}, 30^{\circ} \mathrm{C}, 40^{\circ} \mathrm{C}, 50^{\circ} \mathrm{C}$, $60^{\circ} \mathrm{C}, 70^{\circ} \mathrm{C}$ und $80^{\circ} \mathrm{C}$. Analog hierzu entspricht jeder dargestellte Steigungswert jeweils der Steigung der linearen Regression über die Vorhersagewerte und die zugehörigen Referenzwerte. Beide Werte steigen mit zunehmender Temperatur, so dass entsprechend jeweils das Datenpaar aus RMSEP und Steigungswert einer Temperaturstufe entspricht. Sowohl der RMSEP des Modells über alle Mehlproben, als auch der RMSEP des Modells über den aufgeteilten Datensatz (Erläuterung hierzu siehe Kapitel 5.2), verhalten sich zur zugehörigen Steigung sehr ähnlich. Der Zusammnenhang lässt sich also unabhängig von der Kalibrierart in erster Näherung schreiben als:

$$
\operatorname{RMSEP}\left(\vartheta_{x}\right)=a \cdot \text { Steigung }\left(\vartheta_{x}\right)+b,
$$

womit der lineare Zusammenhang von RMSEP und Steigung der linearen Regression experimentell nachgewiesen werden konnte. 


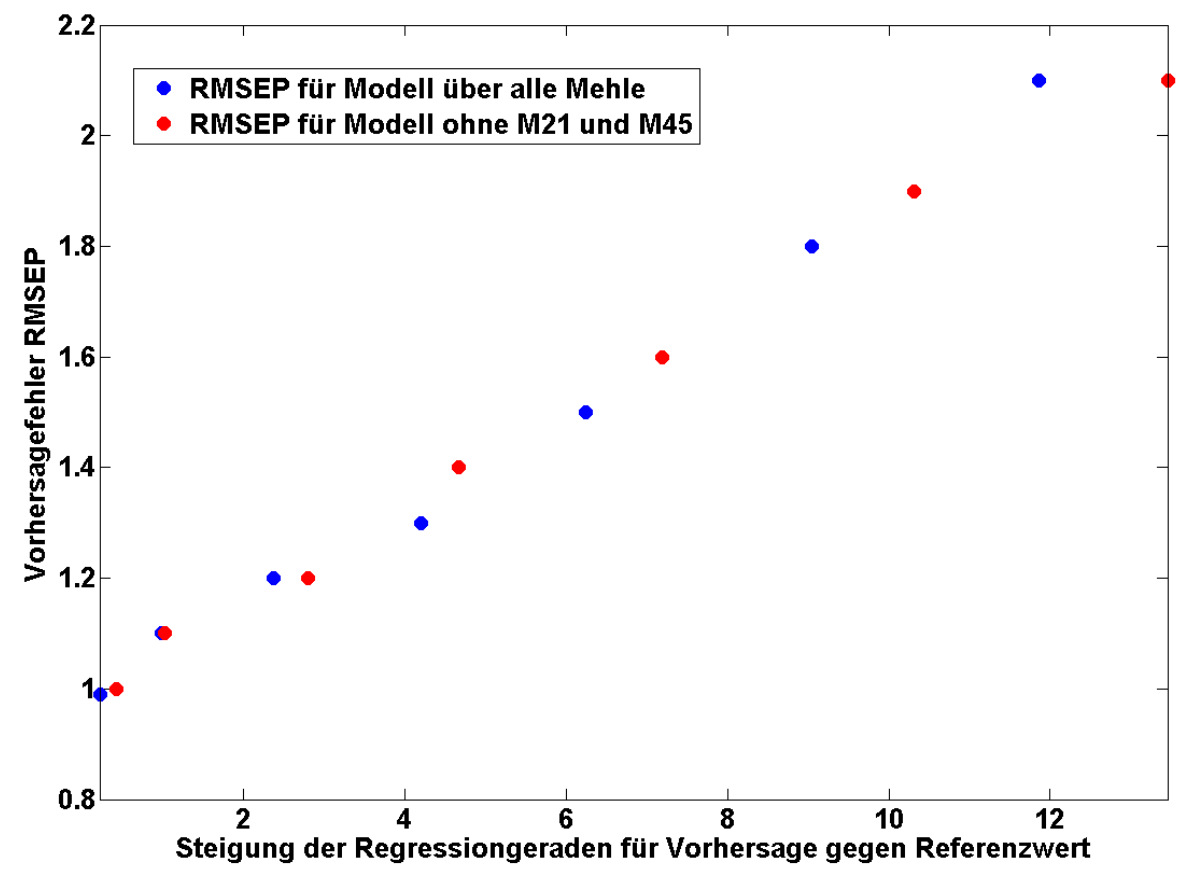

Abbildung 6.1: Zusammenhang des Vorhersagefehlers RMSEP und der Steigung der Vorhersage bei veränderlicher Temperatur sowohl für das Modell über den gesamten Datensatz als auch das Modell über den in Kalibrierund Validierdaten aufgeteilten Datensatz

\subsubsection{Zusammenhang Steigung der linearen Regression und Temperaturvarianz}

Zum weiteren Verständnis der Auswirkung der Temperaturvarianz auf die Vorhersage eines chemometrischen Modells wird nun der Zusammenhang zwischen der Probentemperatur und der Steigung der linearen Regression über den Vorhersage- und den Referenzwert des Modells aus Kapitel 5.2 bei einer Veränderung der Probentemperatur untersucht. Es wurden folglich analog zu Kapitel 5.2 wiederum die Spektren aller Temperaturstufen mit dem $20^{\circ} \mathrm{C}$-Modell vorhergesagt. Die Abbildung 6.2(a) zeigt den Zusammenhang dieser Steigung mit der Probentemperatur für das Kalibriermodell auf Basis des gesamten Kalibrierdatensatzes. Die Daten dieser Betrachtung sind in Tabelle 5.1 auf Seite 82 zu finden. In erster Näherung lässt sich der Zusammenhang der Steigung mit einer anwachsenden Temperatur quadratisch beschreiben. Dies ist sowohl für die Kalibrierung über den gesamten Mehldatensatz als auch für die Kalibrierung über den aufgeteilten Datensatz (siehe Abbildung 6.2(b)), wie er bereits in Kapitel 5.2 beschrieben wurde, zu beobachten. Die Ergebnisse für den aufgeteilten Datensatz sind in Tabelle 5.3 auf Seite 84 zu finden. Der 
Vorhersagefehler verhält sich aufgrund der Beobachtung aus Kapitel 6.1.1 in erster Näherung ebenso quadratisch (siehe Abbildung 6.3). Der Einfluss einer variierenden Probentemperatur auf die Vorhersage eines bei konstanter Temperatur erstellten Kalibriermodells ist unter Betrachtung des Vorhersagefehlers folglich in erster Näherung von quadratischer Natur. Dies bestätigt die Beobachtungen aus Kapitel 5.3, wo bereits durch Erstellung unterschiedlicher Modelle für unterschiedliche Probentemperaturen ein nichtlinearer Einfluss der Probentemperatur auf die Modellbildung aufgrund einer stärkeren Erhöhung der Hauptkomponentenanzahl, als sie für eine lineare Varianz zu erwarten wäre, festgestellt wurde. 

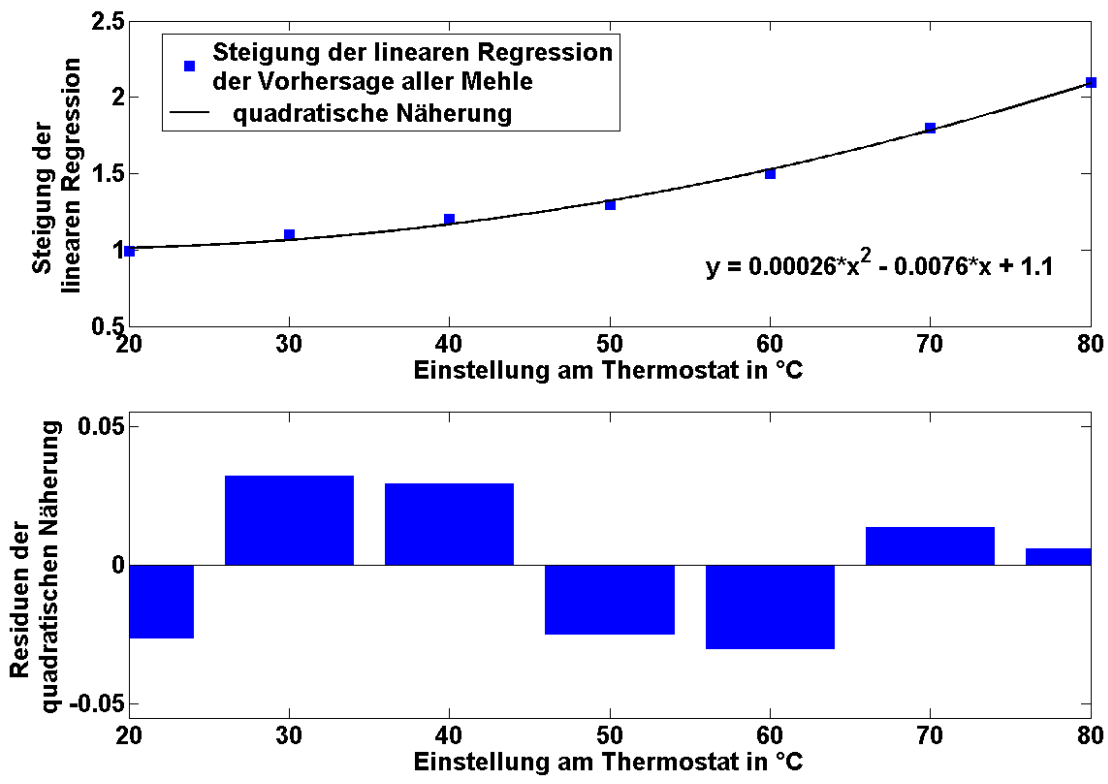

(a) Zusammenhang für das Modell über alle Mehle (Gesamtdatensatz)
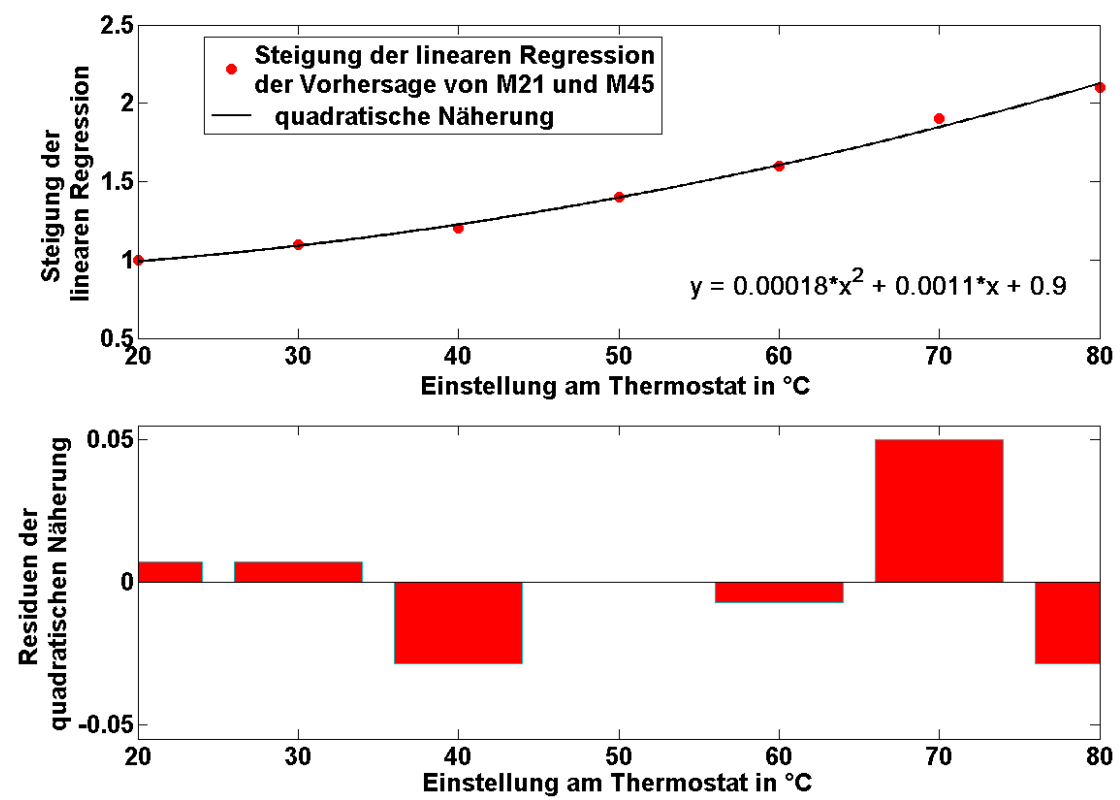

(b) Zusammenhang für das Modell über den aufgeteilten Datensatz (Kalibrier- und Validierdatensatz)

Abbildung 6.2: Steigung der linearen Regressionen der Vorhersagen der beiden $20^{\circ} \mathrm{C}$-Modelle für den aufgeteilten und den Gesamtdatensatz, angewendet auf Spektren bei Temperatureinstellungen am Thermostat von $20^{\circ} \mathrm{C}, 30^{\circ} \mathrm{C}$, $40^{\circ} \mathrm{C}, 50^{\circ} \mathrm{C}, 60^{\circ} \mathrm{C}, 70^{\circ} \mathrm{C}$ und $80^{\circ} \mathrm{C}$; verwendete Mehle M09, M12, M13, M17, M18, M19, M21 und M45 

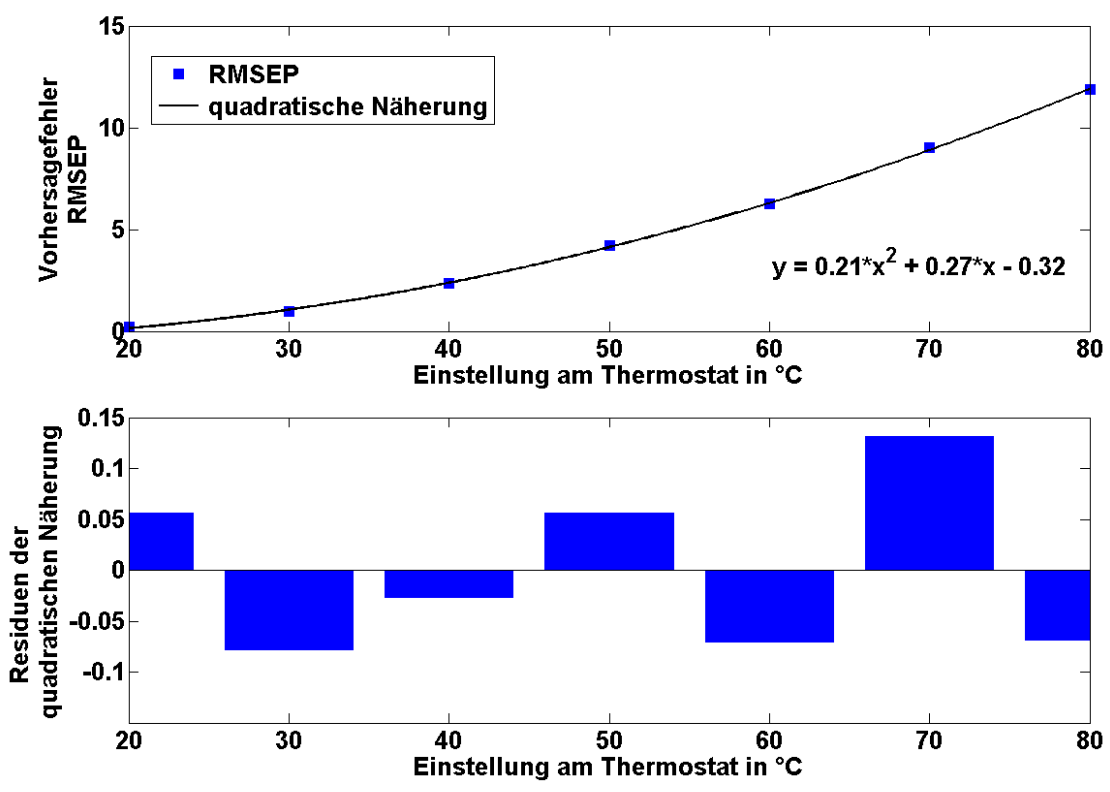

(a) Zusammenhang für das Modell über alle Mehle (Gesamtdatensatz)
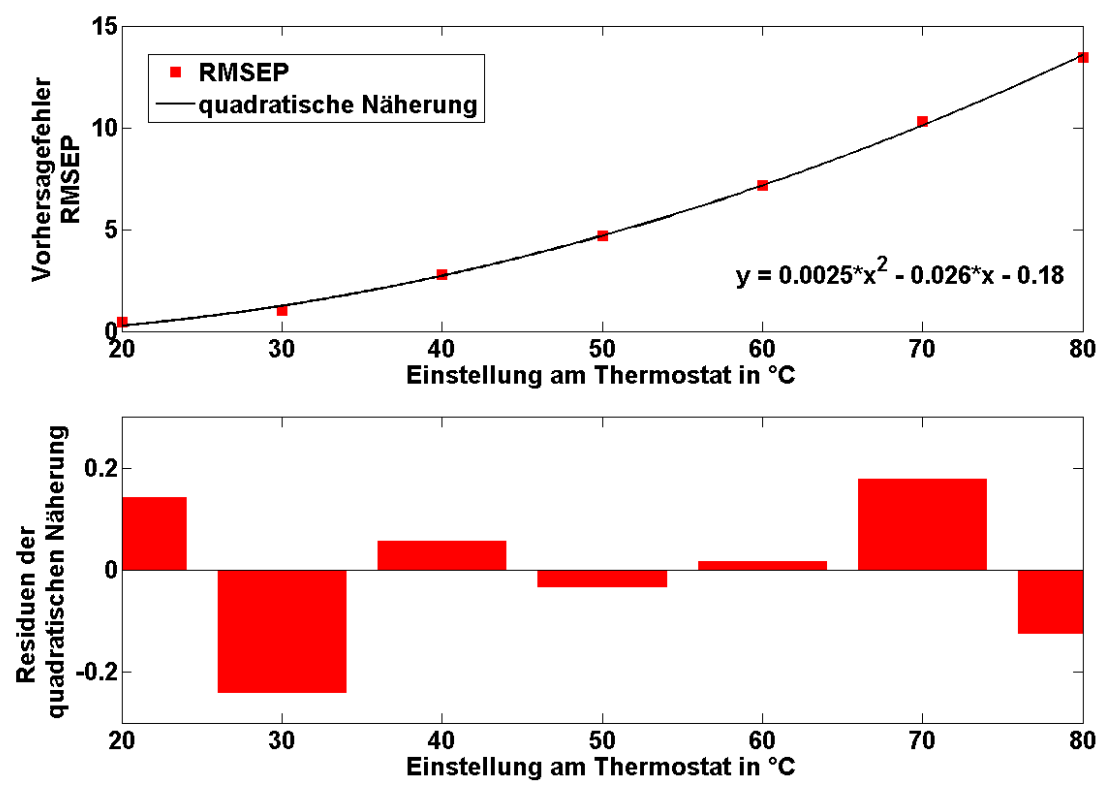

(b) Zusammenhang für das Modell über den aufgeteilten Datensatz (Kalibrier- und Validierdatensatz)

Abbildung 6.3: Vorhersagefehler RMSEP der beiden $20^{\circ} \mathrm{C}$-Modelle für den aufgeteilten und den Gesamtdatensatz, angewendet auf Spektren bei Temperatureinstellungen am Thermostat von $20^{\circ} \mathrm{C}, 30^{\circ} \mathrm{C}, 40^{\circ} \mathrm{C}, 50^{\circ} \mathrm{C}, 60^{\circ} \mathrm{C}$, $70^{\circ} \mathrm{C}$ und $80^{\circ} \mathrm{C}$; verwendete Mehle M09, M12, M13, M17, M18, M19, M21 und M45 


\subsection{Ansatz zur Temperaturkorrektur durch Kalibriertransfer im Referenzwertraum}

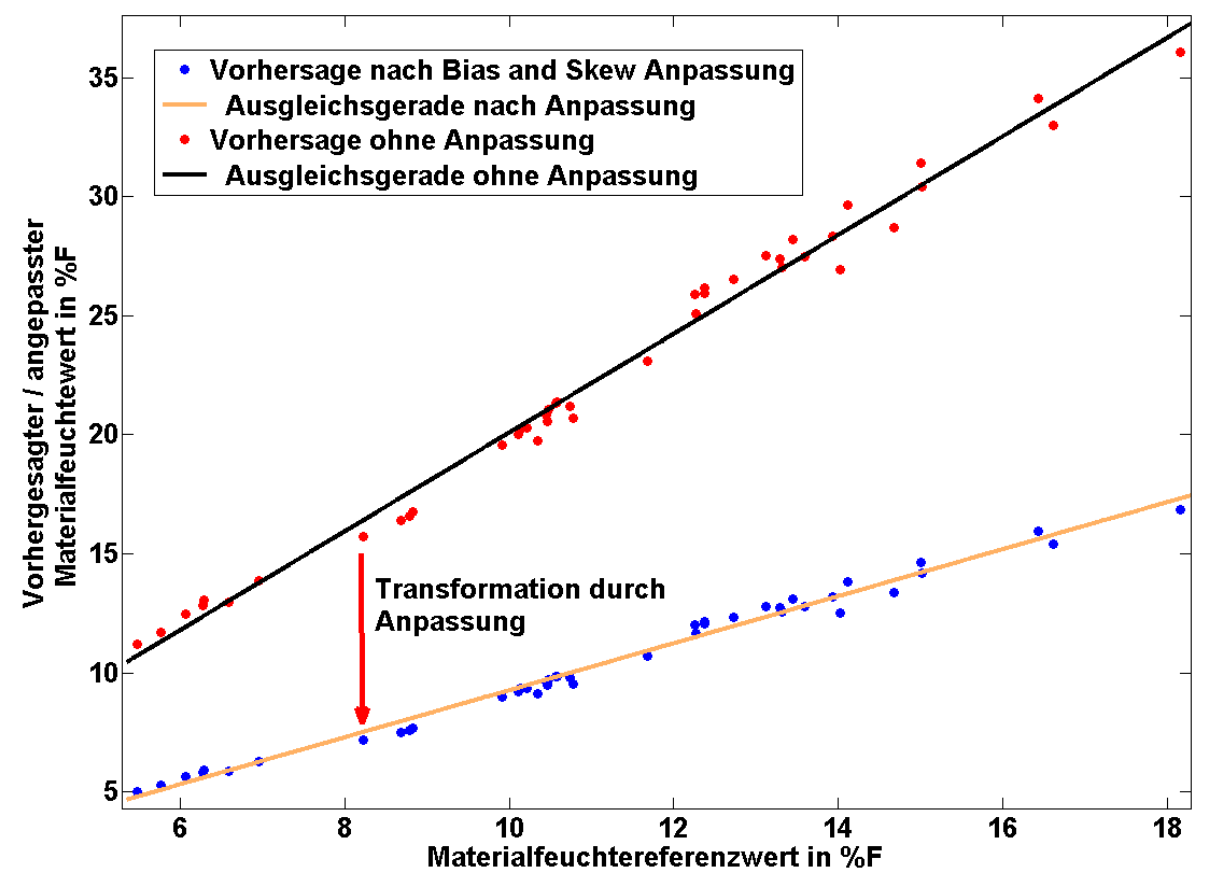

Abbildung 6.4: Exemplarische Darstellung der Anpassung durch „Bias and Skew adjustment" am Komplettdatensatz mit allen Mehlen

Wie bereits in Kapitel 5.2 beschrieben, lässt sich die Abweichung der Vorhersage von Spektren bei einer festen Temperaturstufe mit einem Modell, das für eine andere Temperaturstufe erstellt wurde, auf einen linearen Zusammenhang zurückführen, auch wenn der Zusammenhang, betrachtet über mehrere Temperaturstufen, nicht linear zu beschreiben ist, da die Steigung der linearen Regression nicht linear sondern quadratisch mit der Temperatur anwächst, wie es im Kapitel 6.1.2 gezeigt wurde. Die lineare Abweichung der Vorhersage lässt sich demnach mit Hilfe der linearen Regression wie folgt beschreiben:

$$
M\left(\vartheta_{\text {pre }}\right)=a \cdot M_{\text {ref }}+b
$$

wobei $M_{r e f}$ der Materialfeuchtereferenzwert der jeweiligen Probe und $M\left(\vartheta_{\text {pre }}\right)$ der Vorhersagewert des Kalibriermodells für die Temperaturstufe $\vartheta_{\text {pre }}$ ist. Dieser lineare Zusammenhang lässt die Vermutung zu, dass eine Korrektur der Vorhersage für einzelne Temperaturstufen erreicht werden kann, indem die fehlerhaften Vorhersagewerte $M\left(\vartheta_{\text {pre }}\right)$ durch Umformen der Regressionsgleichung auf die eigentlichen 
Vorhersagewerte der ,ursprünglichen“ Kalibrierung bei Kalibriertemperatur zurücktransformierbar sind. Wird die Gleichung 6.2 umgeformt, so folgt der Zusammenhang:

$$
M_{r e f}=\frac{M\left(\vartheta_{\text {pre }}\right)-b}{a}
$$

Durch eine solche Anpassung der Vorhersagewerte auf Basis der linearen Regression können die fehlerhaften Materialfeuchtewerte auf die Referenzwerte zurücktransformiert werden. Dieses Vorgehen der Anpassung wird in der Literatur als „Bias and Skew adjustment" oder auch „Bias and Skew correction“ bezeichnet und wurde ursprünglich als eine der ersten Kalibriertransfermethoden in der NIR-Spektroskopie eingeführt. Nähere Erläuterungen zum Vorgehen bei dieser Transfermethode sind in dem Buch von Næs et.al. [24] zu finden. Eine Veranschaulichung dieser Methode zeigt Abbildung 6.4.

In Kapitel 5.2 wurden bereits mehrere Vorhersagen mit den beiden Modellen für eine Temperatur von $20^{\circ} \mathrm{C}$ besprochen. Die Vorhersagen wurden zum Einen für den gesamten Mehldatensatz und zum Anderen für einen aufgeteilten Datensatz erstellt. Die Methode des „Bias and Skew adjustment“ wurde exemplarisch an den Vorhersagen für die $80^{\circ} \mathrm{C}$-Temperaturstufe dargestellt. Das Ergebnis zeigt Tabelle 6.1. Zusammenfassend lässt sich für diesen Ansatz der Temperaturkorrektur mit

\begin{tabular}{|c|c|c|c|c|}
\hline 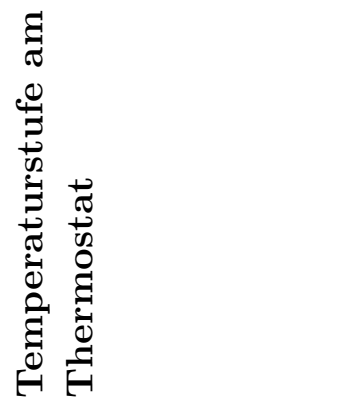 & 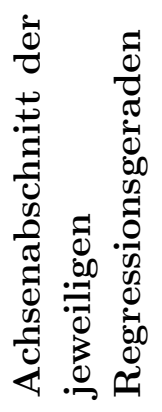 & 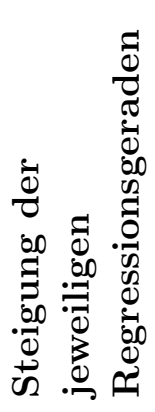 & 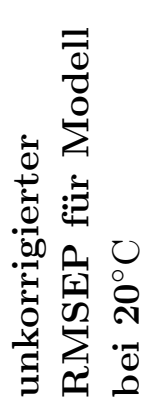 & 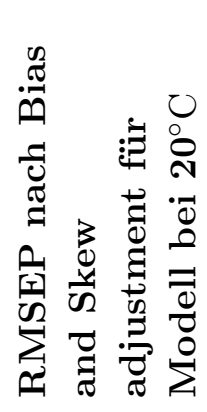 \\
\hline $\begin{array}{l}80^{\circ} \mathrm{C} \\
\text { Modell über alle } \\
\text { Mehle } \\
80^{\circ} \mathrm{C}\end{array}$ & $-0,66$ & 2,1 & 11,859 & 0,837 \\
\hline $\begin{array}{l}\text { Modell über } \\
\text { Kalibrierdatensatz }\end{array}$ & $-0,31$ & 2,1 & 13,454 & 0,412 \\
\hline
\end{tabular}

Tabelle 6.1: Vorhersagefehler RMSEP für die Vorhersage von Spektren bei $80^{\circ} \mathrm{C}$ mit einem Modell für $20^{\circ} \mathrm{C}$ für ein Modell über alle Mehle und ein Modell über einen aufgeteilten Datensatz (Aufteilung siehe Kapitel 5.2)

Hilfe des „Bias and Skew adjusment“ feststellen, dass eine Anpassung mit dieser Methode sehr gut machbar ist und zu überzeugenden Ergebnissen führt, da die Vorhersagefehler nach Anwendung dieser Methode in der gleichen Größenordnung 
des Vorhersagefehlers des jeweiligen Modells auf Spektren, die bei Kalibriertemperatur aufgenommen wurden, liegen (vergleiche hierzu Tabellen 5.1 und 5.3). Jedoch bedeutet dieser Ansatz auch sehr große Einschränkungen in der Robustheit und Zuverlässigkeit eines multivariaten Modells. Außerdem bewirkt dieser Ansatz den Verlust sämtlicher Vorteile der chemometrischen Auswertung. So ist ein solches angepasstes Modell nur so lange gültig, wie keine Veränderungen in der Probenmatrix stattfinden. Bereits kleine Abweichungen von den Anpassungsbedingungen können komplett zum Versagen der Anpassung führen. Zusätzlich gehen alle Sicherheitsmechanismen zur Verhinderung einer Fehlervorhersage wie z. B. die Ausreißerdiagnostik verloren, da eine Ausreißerdiagnostik nur im Spektraldatenraum durchgeführt werden kann. Die Anpassung findet bei diesem Ansatz allerdings nur im Referenzdatenraum statt. Folglich ist dieser Ansatz nur sehr eingeschränkt anwendbar, kann jedoch in Spezialfällen durchaus zu schnellen und akzeptablen Ergebnissen führen.

\subsection{Ansatz zur Temperaturkorrektur über Dotierung des Kalibrierdatensatzes}

Ein weiterer Ansatz zum Umgang mit dem Einfluss einer variierenden Probentemperatur ist die implizite Berücksichtigung der Probentemperatur im Kalibrierdatensatz. Dieser Ansatz benötigt keine Messung der Probentemperatur. Sie wird lediglich im Bereich der zu erwartenden Änderung variiert. Um bei dem bisher betrachteten Beispiel zu bleiben, würde dieser Ansatz zunächst von einem bei $20^{\circ} \mathrm{C}$ erstellten Kalibriermodell ausgehen. Nun sollen jedoch Proben bei einer Temperatur von $80^{\circ} \mathrm{C}$ untersucht und ihr Materialfeuchtewert vorhergesagt werden. Dem vorgeschlagenen Ansatz folgend müssten nun einige Spektren (ca.10-20 Spektren) an voneinander unabhängigen Proben mit variierendem Feuchtegehalt eingemessen werden. Die Spektren dieser in Temperatur und Feuchte variierenden Proben werden anschließend zusammen mit ihren Materialfeuchtereferenzwerten in den bisherigen Kalibrierdatensatz für $20^{\circ} \mathrm{C}$ aufgenommen und es wird ein neues, erweitertes Kalibriermodell entwickelt. Diese Erweiterung oder auch Dotierung des Kalibrierdatensatzes mit Spektren, die bei Zieltemperatur aufgenommen wurden, wird sowohl in der Literatur $[25,88,89]$ als sinnvoller Ansatz vorgeschlagen und auch im praktischen Umfeld der Industrie eingesetzt. Die Tabelle 6.2 zeigt das Ergebnis für die Erweiterung eines Kalibrierdatensatzes bei $20^{\circ} \mathrm{C}$ mit signifikanten Spektren aus einem Datensatz für $80^{\circ} \mathrm{C}$. Außerdem zeigt es zum Vergleich die Kennwerte eines auf den $80^{\circ} \mathrm{C}$-Spektren erstellten Datensatzes. Es wurde die aus Kapitel 5.2 bekannte Einteilung in Kalibrier- und Validierdatensatz verwendet. Demnach wurden die Spektren der gleichen Proben im $20^{\circ} \mathrm{C}$-Kalibrierdatensatz und im $80^{\circ} \mathrm{C}-\mathrm{Kali}-$ brierdatensatz verwendet. Bei dieser Untersuchung lässt sich beobachten, dass der Vorhersagefehler RMSEP des Modells für unbekannte Spektren, also den Validier- 


$\begin{array}{lllll} & & & \\ & & & \end{array}$

Tabelle 6.2: Dotierung des Kalibrierdatensatzes bei $20^{\circ} \mathrm{C}$ mit signifikanten Spektren aus dem $80^{\circ} \mathrm{C}-$ Kalibrierdatensatz (Aufteilung analog zu 5.2)

datensatz, bei dem dotierten $20^{\circ} \mathrm{C}$-Modell größer ist als der Vorhersagefehler für das $80^{\circ} \mathrm{C}$-Modell. Der Kreuzvalidierungsfehler, also der Fehler des Modells, angewendet auf Spektren aus dem Kalibrierdatensatz, verdoppelt sich sogar gegenüber dem Modell für die Zieltemperatur von $80^{\circ} \mathrm{C}$. Dieses Ergebnis zeigt, dass der Ansatz der Erweiterung des Kalibrierdatensatzes um einige Spektren, die um die Varianz der Probentemperatur erweitert wurden, zwar generell möglich ist und auch praktikable Ergebnisse liefert. Allerdings wäre es für eine Anwendung der Temperaturkorrektur wünschenswert, dass der Vorhersagefehler für unbekannte Spektren möglichst gering wird. Der Ansatz der Dotierung zum Transfer einer Kalibrierung hin zu einer neuen Probentemperatur liefert bessere Ergebnisse, wenn die Datenbasis der Spektren bei Zieltemperatur groß wird gegen die Anzahl an Spektren des Urkalibrierdatensatzes. Dies ist in der Realität allerdings selten machbar, da in industriellen Prozessen solche Erweiterungsmessungen meist nur unter sehr großem Aufwand durchgeführt werden können, so dass ein alternativer Ansatz zur Verbesserung der Modelle auf einer geringen Spektrenanzahl bei Zieltemperatur gefunden werden muss. 


\subsection{Ansatz durch Kalibriertransfer des Spektraldatensatzes}

Bisher wurden zwei Ansätze zur Korrektur des Temperatureinflusses untersucht, die beide bereits ihre Anwendung im Routinemessbetrieb bei NIR-spektroskopischen Messaufgaben finden. Jedoch zeigen beide Ansätze Eigenschaften, die noch verbessert werden können. So ist es wünschenswert, dass die Anwendung einer Temperaturkorrektur nicht zum Ausfall der Ausreißerdiagnostik führt, wie es bei Regressionsanpassung (Kapitel 6.2) passiert. Das Fehlen der Ausreißerdiagnostik würde in vielen Bereichen der Lebensmittelindustrie aber auch der chemischen oder pharmazeutischen Industrie zu Ausfällen von kompletten Produktionschargen führen, wenn Fehler im Prozess nicht mehr als Ausreißer erkannt würden. Eine Verbesserung zeigt hier bereits der Ansatz der Kalibrierdatensatzerweiterung mit temperaturvariierten Spektren (Kapitel 6.3), da hier die Ausreißerdiagnostik nicht verloren geht. Jedoch wird das Modell aufgrund der zusätzlichen Varianz der Temperatur ungenauer und auch die Ausreißerdiagnostik wird zwangsläufig unsicherer.

Daher ist ein weiterer Ansatz sinnvoll, der den gesamten Kalibrierdatensatz nicht nur um die Varianz der Probentemperatur erweitert, sondern den kompletten Spektrensatz im Spektraldatenraum derart verschiebt, dass die Spektren im Bereich der neuen zu erwartenden Spektren liegen. Geometrisch gesprochen wird dieser Ansatz die Form der Kalibrierspektren so verändern, als wären sie bereits bei der Zieltemperatur aufgenommen worden. Der hier erläuterte Ansatz verwendet eine Spektrentransformation mit Hilfe der „Piecewise Direct Standardization PDS“.

Zum Transfer der Kalibrierspektren müssen einige Spektren bei der Zieltemperatur eingemessen werden. Anschließend werden zu den neuen Spektren Spektren aus dem Kalibrierdatensatz gesucht, die zueinander passen und somit den gleichen Materialfeuchtereferenzwert zeigen. Diese Spektren werden als Transferspektrenpaare verwendet und auf ihrer Basis wird die Transfermatrix berechnet, die anschließend für die Transferierung des gesamten Kalibrierdatensatzes bei ursprünglicher Temperatur hin zur Zieltemperatur verwendet wird. Diese nun transformierten Spektren werden zusammen mit den bei Zieltemperatur zusätzlich eingemessenen Spektren zu einem vereinten Kalibrierdatensatz zusammengefasst und auf dieser Datenbasis wird ein neues Modell erstellt. Das Ergebnis dieser Vorgehensweise ist in Tabelle 6.3 exemplarisch für einen Kalibriertransfer von $20^{\circ} \mathrm{C}$ zu einer Zieltemperatur von $80^{\circ} \mathrm{C}$ dargestellt. Es wurden verschiedene Ansätze betrachtet. Der Vergleich des transferierten Modells Nr.3 mit dem Modell Nr.2, das für die Zieltemperatur bei $80^{\circ} \mathrm{C}$ erstellt wurde, zeigt ein ähnliches Verhalten des Vorhersagefehlers RMSEP und des Kreuzvalidierungsfehlers $R M S E C V$ wie beim Erweiterungsansatz aus Kapitel 6.3, wobei der $R M S E C V$ bereits leicht verbessert wurde. Es bleibt dabei aber

\footnotetext{
${ }^{1}$ Datenvorbehandlung: 1. Savitzky-Golay-Glättung bei einer Filterweite von 25 Pixeln und einem Polynom 2.Ordnung, 2. standard normal variate (SNV)
} 


\begin{tabular}{lllll} 
MNr. & Modell für & HKz & RMSECV & RMSEP \\
1 & $20^{\circ} \mathrm{C}$ & 6 & 0,179 & 0,399 \\
2 & $80^{\circ} \mathrm{C}$ & 6 & 0,296 & 0,476 \\
3 & $20^{\circ} \mathrm{C} \rightarrow \mathrm{PDS} \rightarrow 80^{\circ} \mathrm{C}$ & 6 & 0,381 & 0,712 \\
4 & $20^{\circ} \mathrm{C} \rightarrow \mathrm{PDS} \rightarrow 80^{\circ} \mathrm{C}$ & 6 & 0,381 & 1,813 \\
5 & $20^{\circ} \mathrm{C} \rightarrow \mathrm{DWPDS} \rightarrow 80^{\circ} \mathrm{C}$ & 6 & 0,415 & 0,462 \\
6 & $20^{\circ} \mathrm{C} \rightarrow \mathrm{DWPDS} \rightarrow 80^{\circ} \mathrm{C}$ & 6 & 0,415 & 1,495 \\
7 & $20^{\circ} \mathrm{C}$ & 6 & 0,179 & 0,399 \\
8 & $80^{\circ} \mathrm{C} \rightarrow 0^{\circ} \mathrm{C}$ & 6 & 0,295 & 0,476 \\
9 & $20^{\circ} \mathrm{C} \rightarrow \mathrm{DWPDS} \rightarrow, 24$ & 0,454 \\
\hline
\end{tabular}

Tabelle 6.3: Anwendung des Kalibriertransfers „Piecewise Direct Standardization“ (PDS), Transformation des $20^{\circ} \mathrm{C}$-Kalibrierdatensatzes auf eine Temperatur von $80^{\circ} \mathrm{C}$, wobei der Kalibrierdatensatz in allen aufgeführten Betrachtungen wiederum der Datensatz aus Kapitel 5.2 ist, entsprechendes gilt für den Validierdatensatz, MNr.: Modellnummer, HKz: Hauptkomponentenzahl, Erläuterung der Modelle in Tabelle 6.4 auf der nächsten Seite.

zu berücksichtigen, dass in diesem Fall der Validierdatensatz auch aus dem transferierten $20^{\circ} \mathrm{C}$-Validierdatensatz bestand. Die Anwendung des Modells Nr. 3 auf den Validierdatensatz, der bei $80^{\circ} \mathrm{C}$ aufgenommen wurde, ist als Modell Nr. 4 dargestellt und zeigt einen sehr großen Vorhersagefehler RMSEP, wobei selbstverständlich der Kreuzvalidierungsfehler $R M S E C V$ gleich bleibt, da sich das eigentliche Modell zwischen Nr. 3 und Nr. 4 nicht ändert. Das Modell Nr. 5 wurde mit einer Erweiterung der PDS erzeugt. Hier kam die „Double Windowed Piecewise Direct Standardization DWPDS“ zum Einsatz. Die Double Windowed Piecewise Direct Standardization hat den Vorteil, dass der Transfer durch die Schachtelung der Fenster noch präziser stattfindet (siehe Kapitel 4.4 auf Seite 72). Das Ergebnis des Modells Nr. 5 muss wiederum mit dem Ergebnis des Modells Nr. 3 verglichen werden, da dieses Modell wiederum auf den zur Zieltemperatur hin transferierten Validierdatensatz angewendet wurde. Es lässt sich feststellen, dass der Kreuzvalidierungsfehler durch die Double Windowed Piecewise Direct Standardization zwar leicht ansteigt, aber noch nicht das Niveau des Modells aus Kapitel 6.3 (siehe Tabelle 6.2 auf Seite 97) erreicht, so dass auch dieser Ansatz immer noch bessere Ergebnisse liefert. Wird nun das Modell Nr. 5 auf den Validierdatensatz bei $80^{\circ} \mathrm{C}$ angewendet, so ergibt sich die Vorgehensweise nach Modell Nr.6. Zwangsläufig bleibt der Kreuzvalidierungsfehler wiederum konstant, da sich das Modell zwischen Nr. 5 und Nr. 6 nicht ändert. Der Vergleich von der Vorgehensweise nach Modell Nr. 6 mit dem Ergebnis des Modells Nr. 4, bei dem das transformierte Modell auch auf den bei $80^{\circ} \mathrm{C}$ aufgenommenen 
MNr. Modell für

$1 \quad 20^{\circ} \mathrm{C}$

$2 \quad 80^{\circ} \mathrm{C}$

$3 \quad 20^{\circ} \mathrm{C} \rightarrow \mathrm{PDS} \rightarrow 80^{\circ} \mathrm{C}$

$4 \quad 20^{\circ} \mathrm{C} \rightarrow \mathrm{PDS} \rightarrow 80^{\circ} \mathrm{C}$

5

6

$7 \quad 20^{\circ} \mathrm{C}$

$8 \quad 80^{\circ} \mathrm{C}$

$9 \quad 20^{\circ} \mathrm{C} \rightarrow$ DWPDS $\rightarrow 80^{\circ} \mathrm{C}$

\section{Anmerkungen / Erläuterungen}

Spektren bei $20^{\circ} \mathrm{C}$, Modell: Kalibrierdatensatz, Vorhersage Validierdatensatz

Spektren bei $80^{\circ} \mathrm{C}$, Modell: Kalibrierdatensatz, Vorhersage Validierdatensatz

Spektren von $20^{\circ} \mathrm{C}$ mit PDS nach $80^{\circ} \mathrm{C}$ transformiert, Modell: umgewandelter Kalibrierdatensatz, Vorhersage Validierdatensatz von $20^{\circ} \mathrm{C}$ auf $80^{\circ} \mathrm{C}$ umgewandelt

Spektren von $20^{\circ} \mathrm{C}$ mit PDS nach $80^{\circ} \mathrm{C}$ transformiert, Modell: umgewandelter Kalibrierdatensatz, Vorhersage Validierdatensatz $80^{\circ} \mathrm{C}$

Spektren von $20^{\circ} \mathrm{C}$ mit DWPDS (Window 15, Subwindow 11) nach $80^{\circ} \mathrm{C}$ transformiert, Modell: umgewandelter Kalibrierdatensatz, Vorhersage Validierdatensatz von $20^{\circ} \mathrm{C}$ auf $80^{\circ} \mathrm{C}$ umgewandelt

Spektren von $20^{\circ} \mathrm{C}$ mit DWPDS (Window 15, Subwindow 11) nach $80^{\circ} \mathrm{C}$ transformiert, Modell: umgewandelter Kalibrierdatensatz, Vorhersage Validierdatensatz $80^{\circ} \mathrm{C}$

Spektren bei $20^{\circ} \mathrm{C}$, Daten wurden vor Modellbildung vorbehandelt ${ }^{1}$, Vorhersage des Validierdatensatzes

Spektren bei $80^{\circ} \mathrm{C}$, Daten wurden vor Modellbildung vorbehandelt ${ }^{1}$, Vorhersage des Validierdatensatzes

Kalibrierdatensatz mit DWPDS nach $80^{\circ} \mathrm{C}$ transformiert, Vorhersage Validierdatensatz original $80^{\circ} \mathrm{C}$-Spektren

Tabelle 6.4: Erläuterung der Modelle aus Tabelle 6.3 auf der vorherigen Seite 
Validierdatensatz angewendet wurde, zeigt einen leichten Anstieg des Kreuzvalidierungsfehlers $R M S E C V$, aber eine Verbesserung des Vorhersagefehlers RMSEP. Aufgrund der Funktionsweise der Piecewise Direct Standardization und auch der Double Windowed Piecewise Direct Standardization lässt sich aber leicht erkennen (siehe auch Kapitel 4.4 auf Seite 72), dass diese beiden Transfermethoden anfällig gegen spektrale Artefakte wie z. B. eine Offsetverschiebung oder eine Verkippung des Spektrums ist, da dies die Form der Spektren maßgeblich beeinflusst und solche Artefakte vom Transferalgorithmus folglich als zu modellierende spektrale Formunterschiede angesehen werden, die aber nicht berücksichtigt werden dürfen, da sie für eine Auswertung nicht informationsrelevant sind und lediglich zu Fehlern führen. Daher wurde zur Verbesserung der Methode zusätzlich eine Datenvorbehandlung vor der eigentlichen Kalibriermodellerstellung durchgeführt. Zunächst wurden die Spektren mit dem Savitzky-Golay-Algorithmus [73] mit einer Fensterweite von 25 Pixeln und einem Polynom zweiten Grades geglättet. Anschließend wurden spektrale Verkippungen und Offests innerhalb des Datensatzes mit der Standard Normal Variate (SNV) entfernt. Auf Basis dieser vorbehandelten Spektren wurden die Modelle Nr. 7 und Nr. 8 erstellt. Der Vergleich dieser beiden Modelle mit den Modellen Nr. 1 und Nr. 2 zeigt, dass sich beide Modelle jeweils gleich verhalten. Dies ist jedoch auch nicht weiter verwunderlich, da die Datenvorbehandlung, die im Fall der Modelle Nr. 7 und 8 vor der Modellerstellung an den Daten selbst durchgeführt wurde, bei der Erstellung der Modelle Nr. 1 und 2 bereits automatisch durchgeführt wurde. Die gleiche Vorgehensweise liegt auch den Modellen Nr.3-6 zu Grunde. Der Vorteil an dem Vorgehen nach Modell Nr. 7 und 8 ist jedoch, dass nun bereits vorbehandelte Daten vor der eingentlichen Modellerstellung zur Verfügung stehen, so dass diese bereinigten Daten auch für die Double Windowed Piecewise Direct Standardization verwendet werden können, was wiederum das Ergebnis dieses Transferalgorithmus verbessert. Wird nun der Kalibrierdatensatz von Modell Nr. 7 mit der Double Windowed Piecewise Direct Standardization und den signifikanten Spektren aus dem $80^{\circ} \mathrm{C}-$ Datensatz analog zu den bisherigen Modelltransfers nach Nr. 3-6 transferiert, ergibt sich das Verhalten nach Modell Nr. 9. Dieses Modell zeigt einen Kreuzvalidierungsfehler $R M S E C V$, der sogar leicht geringer ausfällt, als der $R M S E C V$ des Modells Nr. 8, das auf dem $80^{\circ}$ C-Datensatz erstellt wurde. Sogar der Vorhersagefehler $R M S E P$ des tranferierten Modells Nr. 9 ist ein wenig kleiner als der RMSEP des $80^{\circ} \mathrm{C}$-Modells Nr. 8. Da es sich bei den vorhergesagten Spektren nach Modell Nr. 9 bereits um den Validierdatensatz aus Spektren handelt, die bei der Zieltemperatur $80^{\circ} \mathrm{C}$ aufgenommen wurden, ist gezeigt, dass der neue Ansatz des Spektrentransfers mit Hilfe der Piecewise Direct Standardization und im Besonderen mit Hilfe der Double Windowed Piecewise Direct Standardization die bisherigen Ansätze wie sie in Kapitel 6.2 und Kapitel 6.3 untersucht wurden, ersetzen kann und dabei sogar eine Verbesserung des Vorhersagefehlers ermöglicht.

Der Vorteil dieses Ansatzes ist, dass der Nutzer der NIR-Spektroskopie von seiner bisher etablierten Vorgehensweise, wie sie in Kapitel 6.3 beschrieben ist, nicht ab- 
weichen muss. Er muss weiterhin lediglich wenige Spektren bei der Zieltemperatur einmessen und erstellt auf die gleiche Art seine Kalibrierung. Es wird lediglich ein Rechenschritt zwischengeschaltet, der den Transfer der anzupassenden Kalibrierung in den spektralen Raum der Spektren bei Zieltemperatur verschiebt und somit den Vorhersagefehler minimiert. Bei einer automatisierten Kalibrierung [90] würde der Nutzer somit von dem zusätzlichen Rechenschritt nichts mitbekommen, aber trotz eines unveränderten Vorgehens bei der Neukalibrierung auf die geänderte Temperatur ein sicheres Vorhersageergebnis bekommen. Zusätzlich bleibt die Ausreißerdiagnostik erhalten, da im Gegensatz zum Ansatz der Regressionsanpassung aus Kapitel 6.2 die Veränderung im Spektraldatenraum stattfindet.

Die Untersuchungen dieses Abschnitts zur neuartigen Methode des Kalibriertransfers zusammenfassend lässt sich das folgende Vorgehen daraus ableiten. Zur Übertragung einer Kalibrierung auf eine neue Messtemperatur werden Spektren bei der Zieltemperatur eingemessen. Sowohl diese als auch die Ursprungsspektren werden mit einer bestimmten Datenvorbehandlung von störenden spektralen Artefakten befreit und es werden auf Basis der Referenzwerte Transferspektrenpaare zusammengestellt. Anhand dieser Paare wird die Transfermatrix mit Hilfe der Double Windowed Piecewise Direct Standardization bestimmt und ein neues Modell mit den transferierten Spektren erstellt. Die detaillierte Erläuterung dieser Methode folgt in Kapitel 7.

\subsection{Fazit der Diskussion}

Im Rahmen dieser Arbeit wurden drei verschiedene Ansätze zur Korrektur eines Einflusses durch eine variierende Probentemperatur untersucht, von denen zwei Ansätze bereits in der NIR-Spektroskopie verwendet werden. Es konnte gezeigt werden, dass der Ansatz der Korrektur durch Transfer der Regressionsgleichung der Materialfeuchtevorhersage gegen die Materialfeuchtereferenzwerte zwar funktioniert, aber mit sehr großen Einschränkungen einhergeht, so dass alle Vorteile der multivariaten Auswertung durch chemometrische Algorithmen eingebüßst werden müssen. Im zweiten Schritt konnte gezeigt werden, dass der Ansatz der Erweiterung des Ursprungsspektraldatensatzes um neue Spektren, die bei der Zieltemperatur eingemessen wurden, funktioniert aber noch nicht genügend gute Ergebnisse liefert. Diese Ergebnisse konnten jedoch mit dem neuartigen Ansatz des Kalibriertranfers mit Hilfe der Piecewise Direct Standardization erreicht werden. Es besteht sogar die Möglichkeit, diese Methode durch weitere Untersuchungen noch zu verbessern. Genauer wird hierauf noch einmal in Kapitel 9 eingegangen. 


\section{Kapitel 7}

\section{Methode zur Durchführung der Temperaturkorrektur}

In den Kapiteln 6.2, 6.3 und 6.4 wurden unterschiedliche Ansätze zur Korrektur des Temperatureinflusses in der NIR-Spektroskopie untersucht, die teilweise auch bereits so eingesetzt werden (Kapitel 6.2 und 6.3). In Kapitel 6.4 wurde ein neuer Ansatz vorgeschlagen und untersucht, der die Korrektur der in der Messtemperatur veränderten Spektren selbst ermöglicht, indem der Kalibrierdatensatz im Spektraldatenraum transformiert wird.

Es konnte gezeigt werden, dass dieser Ansatz die Modellqualität eines transferierten Modells liefert, die der Qualität eines bei der Zieltemperatur erstellten Modells entspricht. Somit ermöglicht dieser Ansatz eine signifikante Verbesserung zu den bisherigen Ansätzen. Aus den Erkenntnissen des Kapitels 6.4 lässt sich nun eine Methode zum Vorgehen und sogar zur Automatisierung der Temperaturkorrektur nach diesem Ansatz ableiten. Im Folgenden wird das Vorgehen analog zu den Beispielen aus Kapitel 6.4 erläutert.

Der Datensatz, auf dessen Basis das ursprüngliche Kalibriermodell erstellt wurde, ist ein bei einer Temperatur von $20^{\circ} \mathrm{C}$ aufgenommer Satz von Mehlspektren. Es wird nun davon ausgegangen, dass der Anwender eine Veränderung seiner Messtemperatur hin $\mathrm{zu} 80^{\circ} \mathrm{C}$ erreichen möchte. Als erstes müssen nun wenige Spektren bei der Zieltemperatur eingemessen werden. Damit einhergehend werden gleichzeitig die zugehörigen Referenzwerte dieser Spektren mit Hilfe der Referenzanalytik (für Materialfeuchte siehe Kapitel 2.1) bestimmt. Damit ist die eigentliche Arbeit des Messens bereits erledigt.

Die nächsten Schritte sind nun die Abwandlung der bisherigen Datenauswertung von der Regressionsanpassung oder der Dotierung eines Kalibrierdatensatzes hin zum Spektrentransfer.

Im ersten Schritt zur Erweiterung der Auswertung müssen nun Spektren aus dem Urdatensatz bei $20^{\circ} \mathrm{C}$ ausgewählt werden, die den Spektren bei der Zieltemperatur von $80^{\circ} \mathrm{C}$ möglichst ähnlich sind. Um die ähnlichen Spektren zu klassifizieren, kann der Referenzwert der jeweiligen Spektren verwendet werden. Sind die Referenzwerte gleich, können diese Spektren zu Transferspektrenpaaren zusammengefasst werden. 
Es sollten mindestens 5 Spektrenpaare für diese Auswertungsart gefunden werden. Je größer die Basis der Transferspektrenpaare ist, um so genauer lässt sich ein Datensatz an die neue Temperatur anpassen. Jedoch ist eine Basismenge von ca. 20 Spektrenpaaren ausreichend.

Sind diese Transferspektrenpaare gefunden, kommt der Algorithmus zum Kalibriertransfer zum Einsatz. Mit Hilfe des PDS-Transferalgorithmus (siehe Kapitel 4.4) wird nun eine Transfermatrix bestimmt, die die Verzerrung der bei $20^{\circ} \mathrm{C}$ aufgenommenen Spektren derart ermöglicht, als wären die Spektren bereits bei der Zieltemperatur von $80^{\circ} \mathrm{C}$ aufgenommen worden. Ist die Transfermatrix gefunden, kann der gesamte $20^{\circ} \mathrm{C}$-Datensatz durch Anwendung dieser Matrix umgerechnet werden. Die transferierten Spektren können nun zusammen mit den Spektren, die bei $80^{\circ} \mathrm{C}$ aufgenommen wurden, und den jeweils zugehörigen Referenzwerten zu einem neuen Kalibrierdatensatz zusammengefasst werden. Auf Basis dieses neuen Kalibrierdatensatzes kann nun ein Modell erstellt werden, das zum Einen die Varianzen des Urmodells bei $20^{\circ} \mathrm{C}$ berücksichtigt, und zum Anderen für die Messungen bei einer Temperatur von $80^{\circ} \mathrm{C}$ geeignet ist. Allerdings ist das neue Modell nur für eine Temperatur von $80^{\circ} \mathrm{C}$ geeignet. Spektren bei $20^{\circ} \mathrm{C}$ lassen sich mit diesem Modell nun nicht mehr vorhersagen.

Ist es gewünscht, die $80^{\circ} \mathrm{C}$ Spektren für eine Erweiterung des Urmodells zu verwenden, ohne dass dieses wie in Kapitel 6.3 robust gegen die neue Temperatur von $80^{\circ} \mathrm{C}$ wird, so kann dieser Ansatz selbstverstädlich auch in die andere Richtung verwendet werden.

Es hat sich gezeigt, dass die Spektren vor Bestimmung der Transfermatrix auf jeden Fall mit einer Datenvorbehandlung auf mögliche additive und multiplikative spektrale Effekte korrigiert werden müssen. Der Grund hierfür lässt sich anhand eines einfachen Beispiels leicht verstehen. Es kann sein, dass die Spektren aufgrund leicht unterschiedlicher Messbedingunen, wie z. B. Unterschied im Abstand zwischen Messkopf und Probe, gegeneinander in der Basislinie der Absorbanz verschoben sind. Wird dieser Offset nicht vorher aus dem Datensatz entfernt, würde der Transferalgorithmus diesen Offset als Spektrenvarianz betrachten und diese folglich in der Transfermatrix berücksichtigen. Das würde allerdings zu einer Verfälschung des transferierten Datensatzes führen, da der Offset keine Probenvarianz ist. Die für die Untersuchungen aus Kapitel 6.4 beste Datenvorbehandlung kann in Tabelle 6.3 bei den Modellen 7 und 8 nachgelesen werden.

Ein Veranschaulichung des konkreten Vorgehens für die Bewertung der Ergebnisse aus Kapitel 6.4 und die Methode, wie sie hier vorgeschlagen wird, ist in Abbildung $7.1 \mathrm{zu}$ finden. Die Besonderheit in dieser Abbildung ist die Verwendung eines weiteren Modells, das bei der Zieltemperatur von $80^{\circ} \mathrm{C}$ erstellt wurde. Dieses Modell ist lediglich zur Bewertung des Erfolgs des Kalibriertransfers nötig und wird für die eigentliche Methode im Routinefall nicht mehr benötigt. Zur Bewertung des Transfererfolgs wurden in Abbildung 7.2 beispielhaft zwei Spektren dargestellt, von denen das rot dargestellte Spektrum ein ursprünglich bei $20^{\circ} \mathrm{C}$ aufgenommenenes Spek- 
trum ist, das mit der hier vorgeschlagenen Methode in ein Spektrum verwandelt wurde, als wäre es bei $80^{\circ} \mathrm{C}$ aufgenommen worden. Das blau dargestellte Spektrum ist an der identischen Mehlprobe $\mathrm{M} 21$ bei $80^{\circ} \mathrm{C}$ aufgenommen worden. Im Idealfall sollten diese beiden Spektren folglich identisch sein (vergleiche Abbildung 7.2(a)). Um festzustellen, wie stark die beiden Spektren maximal von einander abweichen, wurde das Differenzspektrum (vergleiche Abbildung 7.2(b)) gebildet. Demnach unterscheiden sich die beiden Spektren in einem Varianzbereich von ca. $\pm 0,06 \mathrm{w}$.E. in der Absorbanz. Dies entspricht bei einem Maximalwert von ca. 2,1 w.E. einer maximalen Varianz von 5,7\%. 


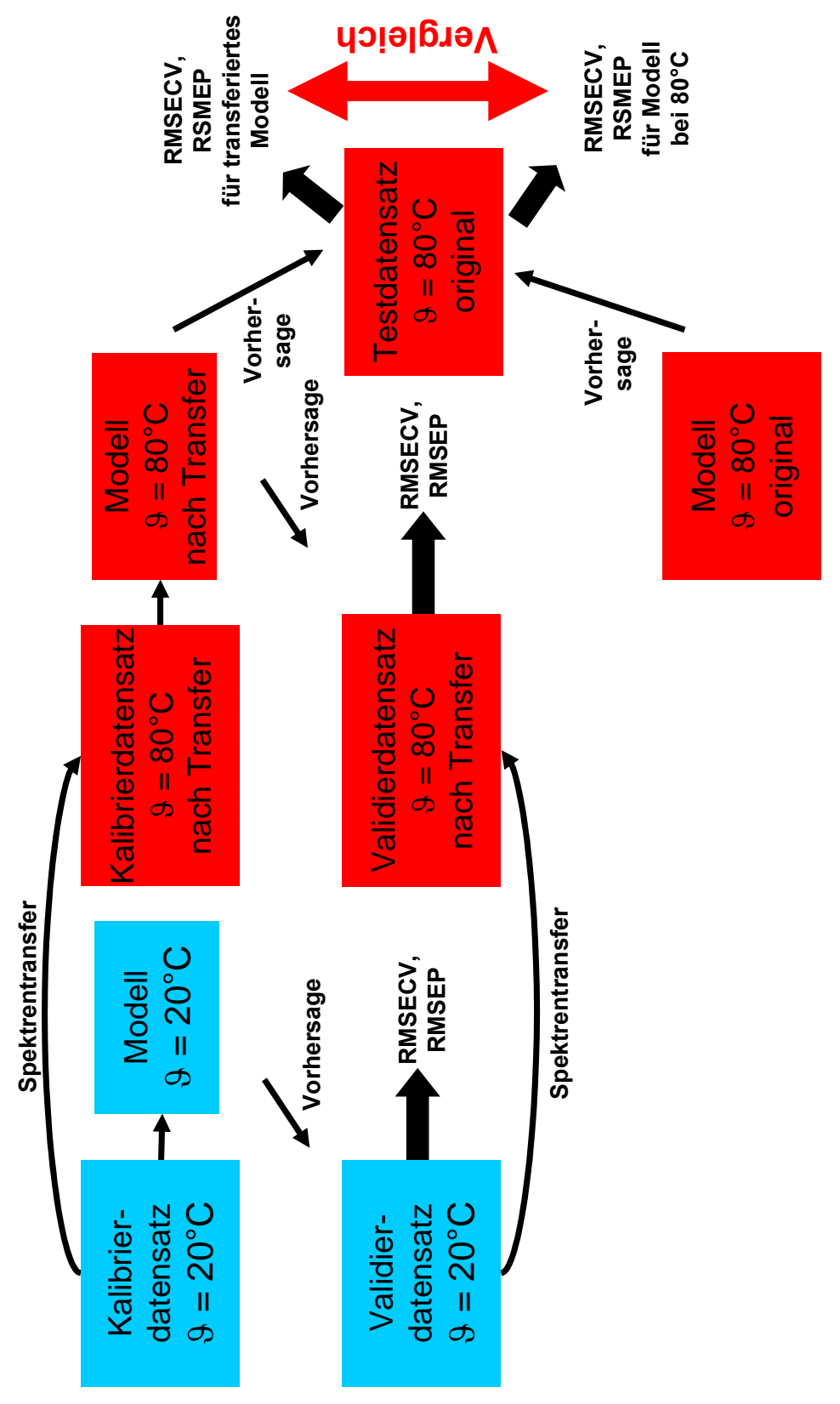




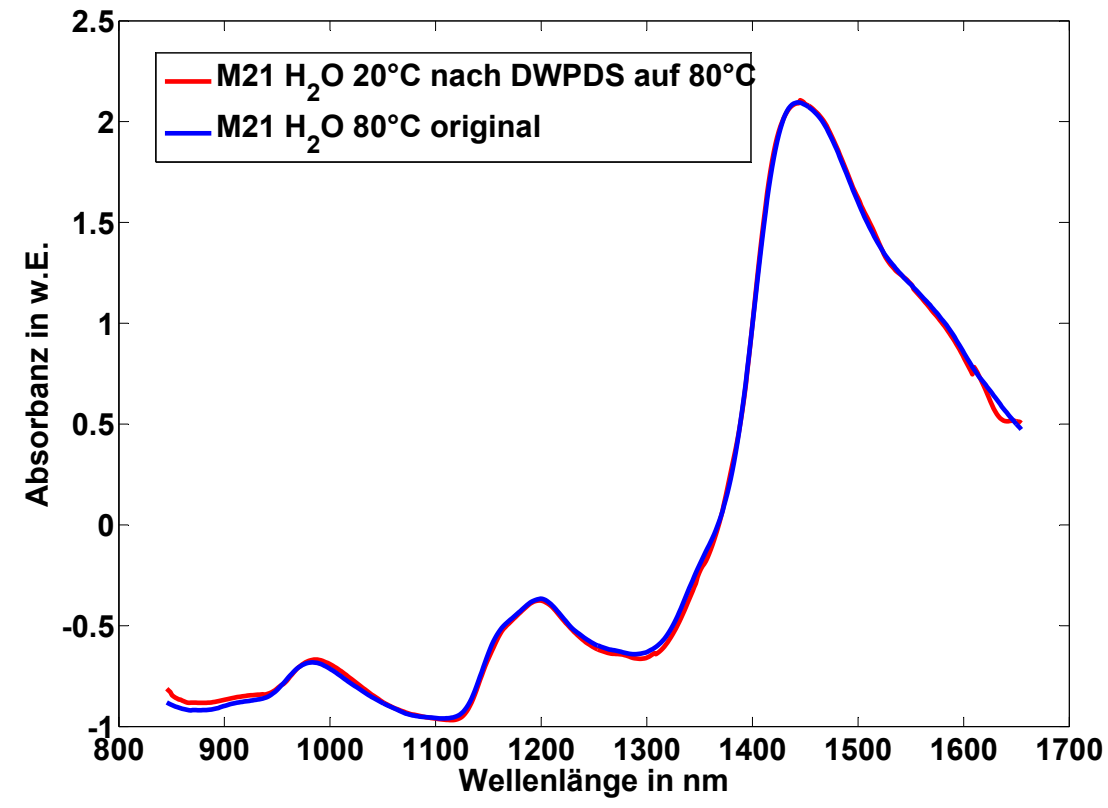

(a) transferiertes und bei $80^{\circ} \mathrm{C}$ gemessenes Spektrum

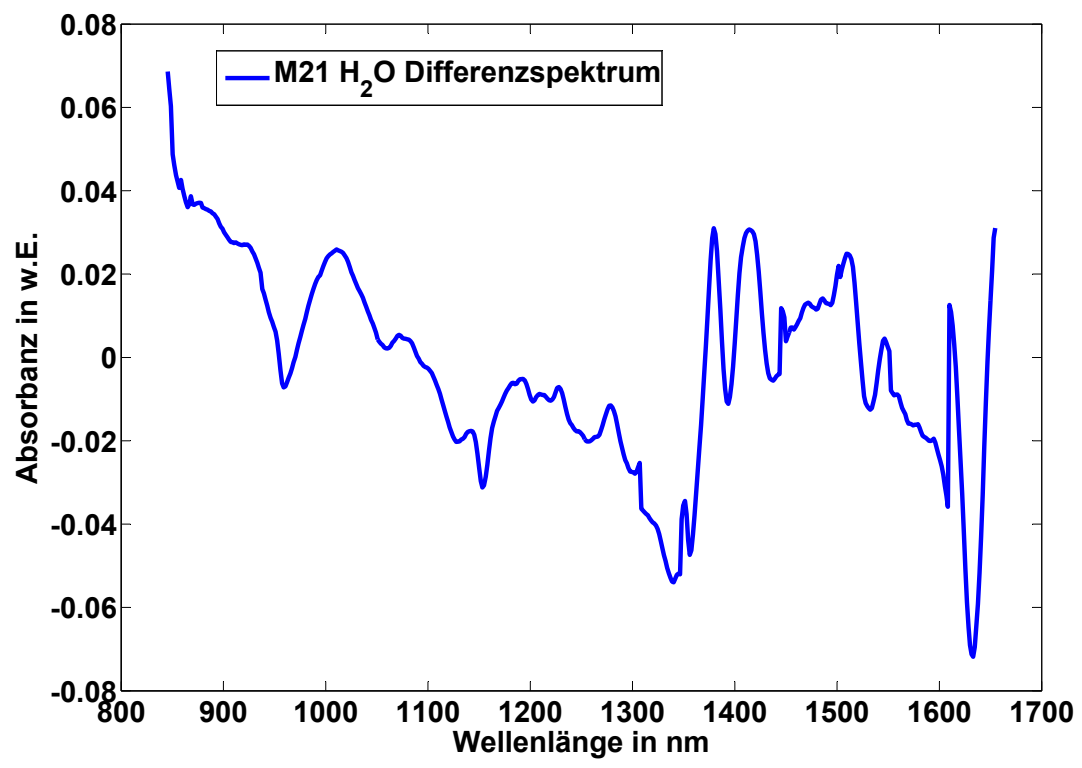

(b) Differenzspektrum der oben dargestellten Spektren

Abbildung 7.2: Vergleich zweier ausgewählter Spektren des Mehls M21 bei der Befeuchtungsart $\mathrm{H}_{2} \mathrm{O}$ (siehe Kapitel 3.6) nach dem Transfer mit Double Windowed Piecewise Direct Standardization von $20^{\circ} \mathrm{C}$ nach $80^{\circ} \mathrm{C}$ mit Differenzspektrum der beiden Spektren 



\section{Kapitel 8}

\section{Zusammenfassung}

Der Markt der Prozessmesstechnik wächst stetig und der Bedarf an universellen Messtechniken zur Qualitätskontrolle wird immer größer. Der wichtigste Parameter einer industriellen Prozesskontrolle ist die Materialfeuchte. Sie spielt eine große Rolle bei Prozessen wie Trocknungen, Mischungsvorgängen, in der Mehlherstellung, bei Kaffeeröstvorgängen usw. Die beste Messtechnik zur Bestimmung der im Produkt auftretenden Materialfeuchte bereits während des laufenden Prozesses ist die NIRSpektroskopie. Die Auswertung von NIR-Spektren ist sehr komplex und bedarf eines äußerst fundierten Wissens über das Vorgehen und die Interpretation während der Auswertung. Das größte bisher nicht gelöste Problem bei dieser Messtechnik ist die Varianz der Probentemperatur während des Prozesses. So können diese Schwankungen nicht nur zu falschen Messergebnissen führen, sondern sogar die komplette Auswertung der Spektren ad absurdum führen. Diese Arbeit hat die Lösung des Temperaturproblems in der NIR-spektroskopischen Messtechnik herbeigeführt. Dazu wurden zunächst die wichtigsten Quereinflüsse, die zu einer Fehlmessung führen können, untersucht und charakterisiert. Auf Basis dieser Erkenntnisse konnte gezeigt werden, welche Einflüsse eine Temperaturvarianz auf die Vorhersage eines chemometrischen Modells zur Auswertung von NIR-Spektren hat. Für diese Charakterisierung wurden Modelle entwickelt, deren Reaktion auf die Temperaturvarianz verglichen wurde. Die bisher ungenügend ausgeführten Ansätze zur Korrektur einer veränderlichen Probentemperatur wurden untersucht und es wurde gezeigt, dass die Ergebnisse dieser Methoden und auch ihre Randbedingungen verbesserungswürdig sind. Es wurde eine neuartige Lösung der Temperaturproblematik gefunden. Hierzu wurde die Piecewise Direct Standardization so in eine Methode eingebunden, dass Spektren des Kalibrierdatensatzes derart in ihrer spektralen Form durch diesen Transferalgorithmus verändert werden, als wären sie bereits bei der Zieltemperatur, für die eine Korrektur benötigt wird, gemessen worden. Die neuartige Methode liefert zum Einen Ergebnisse, als wäre eine Schwankung der Temperatur nie aufgetreten. Zum Anderen hat diese neuentwickelte, universelle Methode den großen Vorteil, dass der Endanwender der Messtechnik sein Vorgehen zur Anpassung einer Kalibrierung in keiner Weise ändern muss. Ganz im Gegenteil kann er sich bei einer zukünftigen Temperaturkorrektur nach wie vor nach den gleichen Arbeitsan- 
weisungen, die er in seinem Prozess festgeschrieben hat, richten. Es wird lediglich ein weiterer, intelligenter und hochkomplexer Auswerteschritt zwischengeschaltet, von dem der Endanwender in einer automatisierten Umgebung nichts mitbekommt. Er wird lediglich die weitaus verbesserten Ergebnisse, die maximierte Universalität und die sehr viel höhere Zuverlässigkeit seiner Prozessmesstechnik bemerken. 


\section{Kapitel 9}

\section{Ausblick}

Im Rahmen dieser Arbeit konnte gezeigt werden, dass die Korrektur des Einflusses einer variierenden Probentemperatur mit einer neuartigen Methode des Kalibriertransfers mit Hilfe der Piecewise Direct Standardization möglich ist. Es hat sich aber auch gezeigt, dass die Genauigkeit des Spektrentransfers noch verbessert werden kann (siehe Ergebnis Kapitel 7). Die Abweichung der transferierten Spektren von den Spektren, die bei Zieltemperatur aufgenommen wurden, also die für die Zieltemperatur zu erwartenden Spektren, kann darin begründet sein, dass neben der Temperaturvarianz noch weitere Varianzen in den Spektren aufgetreten sind. Diese Varianzen müssen in weiterführenden Untersuchungen betrachtet und bewertet werden. Es muss untersucht werden, welchen Einfluss beispielsweise ein unterschiedlicher Fett- oder Proteingehalt zwischen den Spektren der Proben, die jeweils als Spektrentransferpaare zusammengestellt werden, bewirkt. Es hat sich zwar gezeigt, dass bei Mehl solche Einflüsse eine untergeordnete Rolle zu spielen scheinen, da sich die Spektren sehr gut transferieren ließen. Allerdings wird Mehl in der Herstellungsphase auch auf einen konstanten Proteingehalt hin untersucht, so dass die Varianz bei dieser Probenart eher gering sein sollte. Daher sollten die Untersuchungen dieser Arbeit mit zusätzlichem Fokus auf weitere Parameter an weiteren Proben durchgeführt werden. Eine sehr interessante Probe ist in diesem Zusammenhang Kaffee. Der Fettgehalt von Kaffee kann in seinen unterschiedlichen Behandlungsstufen variieren, so dass dieser Bestandteil bei Kaffee möglicherweise durchaus einen Einfluss auf die Transferspektrenpaare haben kann. Auch der Einfluss unterschiedlicher Korngrößen auf die Qualität der vorgeschlagenen Korrekturmethode könnte an Kaffee sehr gut untersucht werden. Nicht zuletzt eine Varianz des Bräunungsgrades, wie er aufgrund der Veränderung der Probentemperatur durchaus möglich ist, könnte einen Einfluss auf die Modellbildung mit diesem Ansatz zeigen. Für diese Untersuchungen wäre Kaffee in gemahlener Form ebenso gut als Probe geeignet. Um eine universelle Anwendbarkeit der Temperaturkorrekturmethode zu überprüfen, müssten Untersuchungen an diversen Proben durchgeführt werden. Dabei wäre es sinnvoll, auch auf eine Varianz der Probenklassen zu achten. Es sollten neben Lebensmittelprodukten auch weitere pulverförmige Proben, wie z. B. Baustoffe oder Produkte der chemischen Industrie untersucht werden. Eine Anwendung dieser Me- 
thode auf pharmazeutische Produkte wäre sicherlich ebenso von sehr großem Interesse. Weiterhin sollte die Anwendbarkeit dieser Methode auch an flüssigen und pastösen Proben untersucht werden, da sich derartige Proben durchaus anders verhalten als pulverförmige Proben. Neben der Varianz der Proben könnte untersucht werden, wie sich die Feuchtevarianz auf die Güte des Spektrentransfers nach dieser Methode auswirkt. So ist es durchaus denkbar, dass Spektren am Rand des Materialfeuchtereferenzbereichs des Modells sich anders verhalten und schlechter tranferiert werden, als Spektren, die einen Referenzwert weiter innen im Referenzwertintervall der Kalibrierung haben. Losgelöst von der Varianz der Materialfeuchte könnten noch weitere Transfermethoden untersucht werden, wie sie in [24, 75, 76, 77] zu finden sind. Möglicherweise könnten Untersuchungen zur Optimierung des Messablaufs für die Suche der Transferspektrenpaare noch zu einer Verbesserung der Transferergebnisse führen. Die Zuordnung der Transferspektrenpaare könnte neben dem Vergleich der Referenzwerte möglicherweise über eine Untersuchung der Spektrenvarianz verbessert werden. So könnte mit Hilfe von Ähnlichkeitsmaßen festgestellt werden, in wieweit sich Spektren aus dem Urdatensatz und dem neuen Datensatz, welcher bei Zieltemperatur für den Transfer aufgenommen wurde, ähneln. In diesem Zusammenhang müssten dann Kriterien gefunden werden, nach denen diese Spektren mit Hinsicht auf die Eignung für einen Transfer klassifiziert werden können. Es sollte eine Untersuchung der statistischen Kennzahlen für die Kriterien zur automatischen Bewertung und Erstellung eines Transfermodells durchgeführt werden, welche Akzeptanzbereiche für diese Parameter festgelegt werden können. Bisher wurde diese Methode an Daten aus einem Versuchsaufbau gewonnen, so dass weiterführende Arbeiten das Ziel haben sollten, diese Ergebnisse und die vorgeschlagene Methode in der Prozesswelt unter realen Bedingungen zu testen und zu validieren. Die Verbesserung der Methode zur Temperaturkorrektur könnte auch durch anpassen der Daten an die Bedingungen der Piecewise Direct Standardization erreicht werden. Die Piecewise Direct Standardization arbeitet mit einem gleitenden Fenster zur Bestimmung der Transfermatrix zwischen den Spektren. Auf Grund dieses Charakters können spektrale Artefakte an den Rändern der transferierten Spektren auftreten, so dass das Ergebnis der Korrekturmethode sicherlich durch Einschränkung des spektralen Bereichs, der zur Auswertung genommen wird, verbessert werden kann. Hierzu müssten die Spektren nach dem Transfer lediglich an den Rändern um jeweils die halbe Fensterbreite des Transferalgorithmus beschnitten werden.

Sind diese Untersuchungen abgeschlossen, sollte die Verwendbarkeit der vorgeschlagenen Methode zur Temperaturkorrektur für weitere Parameter neben der Materialfeuchte untersucht werden. Es sollte durchaus möglich sein, auch Kalibrierungen auf Parameter wie Fett-, Protein-, Stärke-, etc. mit diesem Ansatz in Hinsicht auf den Temperatureinfluss zu korrigieren.

Eine weitere Verbesserung der Temperaturkorrektur könnte eine Anwendung nichtlinearer Ansätze auf diese Problematik liefern. Hierzu müssten verschiedene Methoden wie lokale Modelle (nächste Nachbarn Modelle) [91] oder Cluster Weighted Models 
(CWM) [92] auf ihre Tauglichkeit getestet werden. Es ist durchaus möglich, dass die Zusammenfassung mehrer dieser nichtlinearen Methoden zu sogenannten Ensemblemethoden [93] zu noch besseren Ergebnissen führt. Diese Ergebnisse müssten anschließend mit den Erkenntnissen dieser Arbeit verglichen werden. 



\section{Anhang A}

\section{Probennachweis und Temperaturmesswerte}

\section{A.1 Herkunftsnachweis der verwendeten Mehlproben}

\begin{tabular}{llllll}
\hline $\begin{array}{l}\text { Probenbe- } \\
\text { zeichnung }\end{array}$ & Mehlsorte & Mehltype & Hersteller & Lieferant & $\begin{array}{l}\text { europ. } \\
\text { duktcode }\end{array}$ \\
M09 & Dinkelmehl & Type 1050 & denree & Bioladen & 4021851526309 \\
M12 & Dinkelmehl & Type 630 & Spielberger & Bioladen & 4022381234184 \\
M13 & Dinkelmehl & Type 1050 & Spielberger & Bioladen & 4022381231183 \\
M17 & Weizenmehl & Type 405 & Alnatura & tegut & 4104420021860 \\
M18 & Weizenmehl & Type 550 & Alnatura & tegut & 4104420016125 \\
M19 & Weizenmehl & Type 1050 & Alnatura & tegut & 4104420016149 \\
M21 & Dinkelmehl & Type 630 & Alnatura & tegut & 4104420021884 \\
M45 & Weizenmehl & Type 405 & Goldähren & Aldi & 29005109 \\
\hline
\end{tabular}




\section{A.2 Temperaturmesswerte und Einstellungen des Thermostaten für Mehlmessungen bei Variation der Probentemperatur}

Hinweis Die Bezeichnung der Befeuchtungsarten ist in Kapitel 3.6 und Tabelle 3.5 zu finden.

\begin{tabular}{rlllllll}
\hline $\begin{array}{r}\text { Thermostat } \\
\text { eingestellte Temperatur in }{ }^{\circ} \mathrm{C}\end{array}$ & 20,00 & 30,00 & 40,00 & 50,00 & 60,00 & 70,00 & 80,00 \\
\hline $\begin{array}{r}\text { Mehl M09 getrocknet } \\
\text { gemessene Probentemperatur } \\
\text { in }{ }^{\circ} \mathrm{C}\end{array}$ & 19,75 & 29,48 & 39,25 & 49,06 & 58,77 & 68,53 & 78,23 \\
\hline $\begin{array}{r}\text { Mehl M09 direkt } \\
\text { gemessene Probentemperatur } \\
\text { in }{ }^{\circ} \mathrm{C}\end{array}$ & 19,71 & 29,52 & 39,28 & 48,87 & 58,76 & 68,72 & 78,54 \\
\hline $\begin{array}{r}\text { Mehl M09 F35 } \\
\text { in }{ }^{\circ} \mathrm{C}\end{array}$ & 19,85 & 29,58 & 39,32 & 49,23 & 58,93 & 68,72 & 78,65 \\
gemessene Probentemperatur & & & & & & \\
\hline $\begin{array}{r}\text { Mehl M09 F76 } \\
\text { gemessene Probentemperatur } \\
\text { in }{ }^{\circ} \mathrm{C}\end{array}$ & 19,94 & 29,65 & 39,35 & 49,36 & 58,82 & 68,63 & 78,36 \\
\hline $\begin{array}{r}\text { Mehl M09 H2O } \\
\text { in }{ }^{\circ} \mathrm{C}\end{array}$ & 19,95 & 29,64 & 39,30 & 49,14 & 58,76 & 68,65 & 78,47 \\
\hline gemessene Probentemperatur & & & & & & \\
\hline
\end{tabular}


Mehl M12 getrocknet

am Thermostat eingestellte Temperatur in ${ }^{\circ} \mathrm{C}$ gemessene Probentemperatur in ${ }^{\circ} \mathrm{C}$ $\begin{array}{lllllll}20,00 & 30,00 & 40,00 & 50,00 & 60,00 & 70,00 & 80,00\end{array}$

$19,88 \quad 29,54 \quad 39,31 \quad 49,20 \quad 58,83 \quad 68,58 \quad 78,40$

\begin{tabular}{|c|c|c|c|c|c|c|c|}
\hline Mehl M12 direkt & & & & & & & \\
\hline $\begin{array}{r}\text { am Thermostat eingestellte } \\
\text { Temperatur in }{ }^{\circ} \mathrm{C}\end{array}$ & 20,00 & 30,00 & 40,00 & 50,00 & 60,00 & 70,00 & 80,00 \\
\hline $\begin{array}{r}\text { gemessene } \\
\text { Probentemperatur in }{ }^{\circ} \mathrm{C}\end{array}$ & 19,78 & 29,62 & 39,05 & 49,10 & 58,70 & 68,50 & 78,33 \\
\hline \multicolumn{8}{|l|}{ Mehl M12 F35 } \\
\hline $\begin{array}{r}\text { am Thermostat eingestellte } \\
\text { Temperatur in }{ }^{\circ} \mathrm{C}\end{array}$ & 20,00 & 30,00 & 40,00 & 50,00 & 60,00 & 70,00 & 80,00 \\
\hline $\begin{array}{r}\text { gemessene } \\
\text { Probentemperatur in }{ }^{\circ} \mathrm{C}\end{array}$ & 20,01 & 29,60 & 39,36 & 49,06 & 58,96 & 68,74 & 78,51 \\
\hline \multicolumn{8}{|l|}{ Mehl M12 F55 } \\
\hline $\begin{array}{r}\text { am Thermostat eingestellte } \\
\text { Temperatur in }{ }^{\circ} \mathrm{C} \\
\end{array}$ & 20,00 & 30,00 & 40,00 & 50,00 & 60,00 & 70,00 & 80,00 \\
\hline $\begin{array}{r}\text { gemessene } \\
\text { Probentemperatur in }{ }^{\circ} \mathrm{C} \\
\end{array}$ & 19,78 & 29,45 & 39,30 & 48,97 & 58,77 & 68,58 & 78,32 \\
\hline \multicolumn{8}{|l|}{ Mehl M12 F76 } \\
\hline $\begin{array}{r}\text { am Thermostat eingestellte } \\
\text { Temperatur in }{ }^{\circ} \mathrm{C}\end{array}$ & 20,00 & 30,00 & 40,00 & 50,00 & 60,00 & 70,00 & 80,00 \\
\hline $\begin{array}{r}\text { gemessene } \\
\text { Probentemperatur in }{ }^{\circ} \mathrm{C}\end{array}$ & 19,74 & 29,47 & 39,20 & 49,01 & 58,67 & 68,42 & 78,38 \\
\hline \multicolumn{8}{|l|}{ Mehl M12 H2O } \\
\hline $\begin{array}{r}\text { am Thermostat eingestellte } \\
\text { Temperatur in }{ }^{\circ} \mathrm{C}\end{array}$ & 20,00 & 30,00 & 40,00 & 50,00 & 60,00 & 70,00 & 80,00 \\
\hline $\begin{array}{r}\text { gemessene } \\
\text { Probentemperatur in }{ }^{\circ} \mathrm{C}\end{array}$ & 19,66 & 29,55 & 39,28 & 49,00 & 58,82 & 68,62 & 78,33 \\
\hline
\end{tabular}




\begin{tabular}{|c|c|c|c|c|c|c|c|}
\hline Mehl M13 getrocknet & & & & & & & \\
\hline $\begin{array}{r}\text { am Thermostat eingestellte } \\
\text { Temperatur in }{ }^{\circ} \mathrm{C}\end{array}$ & 20,00 & 30,00 & 40,00 & 50,00 & 60,00 & 70,00 & 80,00 \\
\hline $\begin{array}{r}\text { gemessene } \\
\text { Probentemperatur in }{ }^{\circ} \mathrm{C}\end{array}$ & 19,81 & 29,62 & 39,33 & 49,02 & 58,68 & 68,49 & 78,16 \\
\hline \multicolumn{8}{|l|}{ Mehl M13 direkt } \\
\hline $\begin{array}{r}\text { am Thermostat eingestellte } \\
\text { Temperatur in }{ }^{\circ} \mathrm{C}\end{array}$ & 20,00 & 30,00 & 40,00 & 50,00 & 60,00 & 70,00 & 80,00 \\
\hline $\begin{array}{r}\text { gemessene } \\
\text { Probentemperatur in }{ }^{\circ} \mathrm{C}\end{array}$ & 19,70 & 29,58 & 39,41 & 49,09 & 58,80 & 68,71 & 78,56 \\
\hline \multicolumn{8}{|l|}{ Mehl M13 F35 } \\
\hline $\begin{array}{r}\text { am Thermostat eingestellte } \\
\text { Temperatur in }{ }^{\circ} \mathrm{C}\end{array}$ & 20,00 & 30,00 & 40,00 & 50,00 & 60,00 & 70,00 & 80,00 \\
\hline $\begin{array}{r}\text { gemessene } \\
\text { Probentemperatur in }{ }^{\circ} \mathrm{C}\end{array}$ & 19,87 & 29,70 & 39,53 & 49,15 & 58,91 & 68,75 & 78,47 \\
\hline \multicolumn{8}{|l|}{ Mehl M13 F76 } \\
\hline $\begin{array}{r}\text { am Thermostat eingestellte } \\
\text { Temperatur in }{ }^{\circ} \mathrm{C}\end{array}$ & 20,00 & 30,00 & 40,00 & 50,00 & 60,00 & 70,00 & 80,00 \\
\hline $\begin{array}{r}\text { gemessene } \\
\text { Probentemperatur in }{ }^{\circ} \mathrm{C}\end{array}$ & 19,90 & 29,70 & 39,50 & 49,16 & 58,72 & 68,61 & 78,28 \\
\hline \multicolumn{8}{|l|}{ Mehl M13 H2O } \\
\hline $\begin{array}{r}\text { am Thermostat eingestellte } \\
\text { Temperatur in }{ }^{\circ} \mathrm{C}\end{array}$ & 20,00 & 30,00 & 40,00 & 50,00 & 60,00 & 70,00 & 80,00 \\
\hline $\begin{array}{r}\text { gemessene } \\
\text { Probentemperatur in }{ }^{\circ} \mathrm{C}\end{array}$ & 19,90 & 29,58 & 39,35 & 49,03 & 58,78 & 68,54 & 78,28 \\
\hline
\end{tabular}


Mehl M17 getrocknet

am Thermostat eingestellte Temperatur in ${ }^{\circ} \mathrm{C}$ gemessene Probentemperatur in ${ }^{\circ} \mathrm{C}$ $\begin{array}{lllllll}20,00 & 30,00 & 40,00 & 50,00 & 60,00 & 70,00 & 80,00\end{array}$

$\begin{array}{lllllll}19,92 & 29,48 & 39,44 & 49,30 & 59,03 & 68,77 & 78,60\end{array}$

\begin{tabular}{|c|c|c|c|c|c|c|c|}
\hline Mehl M17 direkt & & & & & & & \\
\hline $\begin{array}{r}\text { am Thermostat eingestellte } \\
\text { Temperatur in }{ }^{\circ} \mathrm{C}\end{array}$ & 20,00 & 30,00 & 40,00 & 50,00 & 60,00 & 70,00 & 80,00 \\
\hline $\begin{array}{r}\text { gemessene } \\
\text { Probentemperatur in }{ }^{\circ} \mathrm{C}\end{array}$ & 19,84 & 29,80 & 39,20 & 49,20 & 58,90 & 68,72 & 78,57 \\
\hline \multicolumn{8}{|l|}{ Mehl M17 F35 } \\
\hline $\begin{array}{r}\text { am Thermostat eingestellte } \\
\text { Temperatur in }{ }^{\circ} \mathrm{C}\end{array}$ & 20,00 & 30,00 & 40,00 & 50,00 & 60,00 & 70,00 & 80,00 \\
\hline $\begin{array}{r}\text { gemessene } \\
\text { Probentemperatur in }{ }^{\circ} \mathrm{C}\end{array}$ & 20,06 & 29,73 & 39,42 & 49,11 & 59,11 & 68,80 & 78,62 \\
\hline \multicolumn{8}{|l|}{ Mehl M17 F55 } \\
\hline $\begin{array}{r}\text { am Thermostat eingestellte } \\
\text { Temperatur in }{ }^{\circ} \mathrm{C}\end{array}$ & 20,00 & 30,00 & 40,00 & 50,00 & 60,00 & 70,00 & 80,00 \\
\hline $\begin{array}{r}\text { gemessene } \\
\text { Probentemperatur in }{ }^{\circ} \mathrm{C}\end{array}$ & 19,91 & 29,54 & 39,35 & 49,20 & 58,97 & 68,61 & 78,51 \\
\hline \multicolumn{8}{|l|}{ Mehl M17 F76 } \\
\hline $\begin{array}{r}\text { am Thermostat eingestellte } \\
\text { Temperatur in }{ }^{\circ} \mathrm{C}\end{array}$ & 20,00 & 30,00 & 40,00 & 50,00 & 60,00 & 70,00 & 80,00 \\
\hline $\begin{array}{r}\text { gemessene } \\
\text { Probentemperatur in }{ }^{\circ} \mathrm{C}\end{array}$ & 19,77 & 29,38 & 39,15 & 49,00 & 58,76 & 68,50 & 78,53 \\
\hline \multicolumn{8}{|l|}{ Mehl M17 H2O } \\
\hline $\begin{array}{r}\text { am Thermostat eingestellte } \\
\text { Temperatur in }{ }^{\circ} \mathrm{C}\end{array}$ & 20,00 & 30,00 & 40,00 & 50,00 & 60,00 & 70,00 & 80,00 \\
\hline $\begin{array}{r}\text { gemessene } \\
\text { Probentemperatur in }{ }^{\circ} \mathrm{C}\end{array}$ & 19,73 & 29,64 & 39,30 & 49,05 & 58,89 & 68,90 & 78,54 \\
\hline
\end{tabular}




\begin{tabular}{|c|c|c|c|c|c|c|c|}
\hline \multirow{2}{*}{$\begin{array}{r}\text { Mehl M18 getrocknet } \\
\text { am Thermostat eingestellte } \\
\text { Temperatur in }{ }^{\circ} \mathrm{C}\end{array}$} & \multirow[b]{2}{*}{20,00} & \multirow[b]{2}{*}{30,00} & \multirow[b]{2}{*}{40,00} & \multirow[b]{2}{*}{50,00} & \multirow[b]{2}{*}{60,00} & \multirow[b]{2}{*}{70,00} & \multirow[b]{2}{*}{80,00} \\
\hline & & & & & & & \\
\hline $\begin{array}{r}\text { gemessene } \\
\text { Probentemperatur in }{ }^{\circ} \mathrm{C}\end{array}$ & 19,90 & 29,50 & 39,35 & 49,22 & 58,82 & 68,53 & 78,40 \\
\hline \multicolumn{8}{|l|}{ Mehl M18 direkt } \\
\hline $\begin{array}{r}\text { am Thermostat eingestellte } \\
\text { Temperatur in }{ }^{\circ} \mathrm{C}\end{array}$ & 20,00 & 30,00 & 40,00 & 50,00 & 60,00 & 70,00 & 80,00 \\
\hline $\begin{array}{r}\text { gemessene } \\
\text { Probentemperatur in }{ }^{\circ} \mathrm{C}\end{array}$ & 19,80 & 29,74 & 39,22 & 49,06 & 58,85 & 68,61 & 78,40 \\
\hline \multicolumn{8}{|l|}{ Mehl M18 F35 } \\
\hline $\begin{array}{r}\text { am Thermostat eingestellte } \\
\text { Temperatur in }{ }^{\circ} \mathrm{C}\end{array}$ & 20,00 & 30,00 & 40,00 & 50,00 & 60,00 & 70,00 & 80,00 \\
\hline $\begin{array}{r}\text { gemessene } \\
\text { Probentemperatur in }{ }^{\circ} \mathrm{C}\end{array}$ & 20,04 & 29,66 & 39,35 & 48,98 & 58,86 & 68,62 & 78,54 \\
\hline \multicolumn{8}{|l|}{ Mehl M18 F55 } \\
\hline $\begin{array}{r}\text { am Thermostat eingestellte } \\
\text { Temperatur in }{ }^{\circ} \mathrm{C}\end{array}$ & 20,00 & 30,00 & 40,00 & 50,00 & 60,00 & 70,00 & 80,00 \\
\hline $\begin{array}{r}\text { gemessene } \\
\text { Probentemperatur in }{ }^{\circ} \mathrm{C}\end{array}$ & 19,88 & 29,48 & 39,29 & 49,07 & 58,76 & 68,42 & 78,26 \\
\hline \multicolumn{8}{|l|}{ Mehl M18 F76 } \\
\hline $\begin{array}{r}\text { am Thermostat eingestellte } \\
\text { Temperatur in }{ }^{\circ} \mathrm{C}\end{array}$ & 20,00 & 30,00 & 40,00 & 50,00 & 60,00 & 70,00 & 80,00 \\
\hline $\begin{array}{r}\text { gemessene } \\
\text { Probentemperatur in }{ }^{\circ} \mathrm{C}\end{array}$ & 19,80 & 29,46 & 39,21 & 48,97 & 58,63 & 68,44 & 78,28 \\
\hline \multicolumn{8}{|l|}{ Mehl M18 H2O } \\
\hline $\begin{array}{r}\text { am Thermostat eingestellte } \\
\text { Temperatur in }{ }^{\circ} \mathrm{C}\end{array}$ & 20,00 & 30,00 & 40,00 & 50,00 & 60,00 & 70,00 & 80,00 \\
\hline $\begin{array}{r}\text { gemessene } \\
\text { Probentemperatur in }{ }^{\circ} \mathrm{C}\end{array}$ & 19,79 & 29,50 & 39,22 & 48,97 & 58,60 & 68,52 & 78,22 \\
\hline
\end{tabular}




\begin{tabular}{|c|c|c|c|c|c|c|c|}
\hline \multirow{2}{*}{$\begin{array}{r}\text { Mehl M19 getrocknet } \\
\text { am Thermostat eingestellte } \\
\text { Temperatur in }{ }^{\circ} \mathrm{C}\end{array}$} & \multirow[b]{2}{*}{20,00} & \multirow[b]{2}{*}{30,00} & \multirow[b]{2}{*}{40,00} & \multirow[b]{2}{*}{50,00} & \multirow[b]{2}{*}{60,00} & \multirow[b]{2}{*}{70,00} & \multirow[b]{2}{*}{80,00} \\
\hline & & & & & & & \\
\hline $\begin{array}{r}\text { gemessene } \\
\text { Probentemperatur in }{ }^{\circ} \mathrm{C}\end{array}$ & 19,93 & 29,74 & 39,38 & 49,29 & 59,10 & 68,76 & 78,50 \\
\hline \multicolumn{8}{|l|}{ Mehl M19 direkt } \\
\hline $\begin{array}{r}\text { am Thermostat eingestellte } \\
\text { Temperatur in }{ }^{\circ} \mathrm{C}\end{array}$ & 20,00 & 30,00 & 40,00 & 50,00 & 60,00 & 70,00 & 80,00 \\
\hline $\begin{array}{r}\text { gemessene } \\
\text { Probentemperatur in }{ }^{\circ} \mathrm{C}\end{array}$ & 19,74 & 29,53 & 39,24 & 49,05 & 59,89 & 68,47 & 78,12 \\
\hline \multicolumn{8}{|l|}{ Mehl M19 F35 } \\
\hline $\begin{array}{r}\text { am Thermostat eingestellte } \\
\text { Temperatur in }{ }^{\circ} \mathrm{C}\end{array}$ & 20,00 & 30,00 & 40,00 & 50,00 & 60,00 & 70,00 & 80,00 \\
\hline $\begin{array}{r}\text { gemessene } \\
\text { Probentemperatur in }{ }^{\circ} \mathrm{C}\end{array}$ & 19,63 & 29,39 & 39,14 & 48,94 & 58,70 & 68,49 & 78,14 \\
\hline \multicolumn{8}{|l|}{ Mehl M19 F55 } \\
\hline $\begin{array}{r}\text { am Thermostat eingestellte } \\
\text { Temperatur in }{ }^{\circ} \mathrm{C}\end{array}$ & 20,00 & 30,00 & 40,00 & 50,00 & 60,00 & 70,00 & 80,00 \\
\hline $\begin{array}{r}\text { gemessene } \\
\text { Probentemperatur in }{ }^{\circ} \mathrm{C}\end{array}$ & 19,79 & 29,34 & 39,20 & 49,00 & 58,82 & 68,64 & 78,42 \\
\hline \multicolumn{8}{|l|}{ Mehl M19 F76 } \\
\hline $\begin{array}{r}\text { am Thermostat eingestellte } \\
\text { Temperatur in }{ }^{\circ} \mathrm{C}\end{array}$ & 20,00 & 30,00 & 40,00 & 50,00 & 60,00 & 70,00 & 80,00 \\
\hline $\begin{array}{r}\text { gemessene } \\
\text { Probentemperatur in }{ }^{\circ} \mathrm{C}\end{array}$ & 19,82 & 29,40 & 39,22 & 48,97 & 58,76 & 68,58 & 78,18 \\
\hline \multicolumn{8}{|l|}{ Mehl M19 H2O } \\
\hline $\begin{array}{r}\text { am Thermostat eingestellte } \\
\text { Temperatur in }{ }^{\circ} \mathrm{C}\end{array}$ & 20,00 & 30,00 & 40,00 & 50,00 & 60,00 & 70,00 & 80,00 \\
\hline $\begin{array}{r}\text { gemessene } \\
\text { Probentemperatur in }{ }^{\circ} \mathrm{C}\end{array}$ & 19,80 & 29,31 & 39,08 & 48,98 & 58,73 & 68,40 & 78,08 \\
\hline
\end{tabular}




\begin{tabular}{|c|c|c|c|c|c|c|c|}
\hline \multirow{2}{*}{$\begin{array}{r}\text { Mehl M21 getrocknet } \\
\text { am Thermostat eingestellte } \\
\text { Temperatur in }{ }^{\circ} \mathrm{C}\end{array}$} & \multirow[b]{2}{*}{20,00} & \multirow[b]{2}{*}{30,00} & \multirow[b]{2}{*}{40,00} & \multirow[b]{2}{*}{50,00} & \multirow[b]{2}{*}{60,00} & \multirow[b]{2}{*}{70,00} & \multirow[b]{2}{*}{80,00} \\
\hline & & & & & & & \\
\hline $\begin{array}{r}\text { gemessene } \\
\text { Probentemperatur in }{ }^{\circ} \mathrm{C}\end{array}$ & 19,86 & 29,59 & 39,23 & 49,15 & 58,98 & 68,58 & 78,43 \\
\hline \multicolumn{8}{|l|}{ Mehl M21 direkt } \\
\hline $\begin{array}{r}\text { am Thermostat eingestellte } \\
\text { Temperatur in }{ }^{\circ} \mathrm{C}\end{array}$ & 20,00 & 30,00 & 40,00 & 50,00 & 60,00 & 70,00 & 80,00 \\
\hline $\begin{array}{r}\text { gemessene } \\
\text { Probentemperatur in }{ }^{\circ} \mathrm{C}\end{array}$ & 19,77 & 29,59 & 39,46 & 48,90 & 58,92 & 68,84 & 78,83 \\
\hline \multicolumn{8}{|l|}{ Mehl M21 F35 } \\
\hline $\begin{array}{r}\text { am Thermostat eingestellte } \\
\text { Temperatur in }{ }^{\circ} \mathrm{C}\end{array}$ & 20,00 & 30,00 & 40,00 & 50,00 & 60,00 & 70,00 & 80,00 \\
\hline $\begin{array}{r}\text { gemessene } \\
\text { Probentemperatur in }{ }^{\circ} \mathrm{C}\end{array}$ & 19,98 & 29,66 & 39,34 & 49,33 & 59,11 & 68,75 & 78,72 \\
\hline \multicolumn{8}{|l|}{ Mehl M21 F76 } \\
\hline $\begin{array}{r}\text { am Thermostat eingestellte } \\
\text { Temperatur in }{ }^{\circ} \mathrm{C}\end{array}$ & 20,00 & 30,00 & 40,00 & 50,00 & 60,00 & 70,00 & 80,00 \\
\hline $\begin{array}{r}\text { gemessene } \\
\text { Probentemperatur in }{ }^{\circ} \mathrm{C}\end{array}$ & 19,95 & 29,71 & 39,35 & 49,33 & 59,20 & 68,85 & 78,43 \\
\hline \multicolumn{8}{|l|}{ Mehl M21 H2O } \\
\hline $\begin{array}{r}\text { am Thermostat eingestellte } \\
\text { Temperatur in }{ }^{\circ} \mathrm{C}\end{array}$ & 20,00 & 30,00 & 40,00 & 50,00 & 60,00 & 70,00 & 80,00 \\
\hline $\begin{array}{r}\text { gemessene } \\
\text { Probentemperatur in }{ }^{\circ} \mathrm{C}\end{array}$ & 19,92 & 29,60 & 39,34 & 49,25 & 59,08 & 68,74 & 78,57 \\
\hline
\end{tabular}




\begin{tabular}{|c|c|c|c|c|c|c|c|}
\hline Mehl M45 getrocknet & & & & & & & \\
\hline $\begin{array}{r}\text { am Thermostat eingestellte } \\
\text { Temperatur in }{ }^{\circ} \mathrm{C}\end{array}$ & 20,00 & 30,00 & 40,00 & 50,00 & 60,00 & 70,00 & 80,00 \\
\hline $\begin{array}{r}\text { gemessene } \\
\text { Probentemperatur in }{ }^{\circ} \mathrm{C}\end{array}$ & 19,84 & 29,54 & 39,39 & 49,10 & 58,92 & 68,72 & 78,40 \\
\hline \multicolumn{8}{|l|}{ Mehl M45 direkt } \\
\hline $\begin{array}{r}\text { am Thermostat eingestellte } \\
\text { Temperatur in }{ }^{\circ} \mathrm{C}\end{array}$ & 20,00 & 30,00 & 40,00 & 50,00 & 60,00 & 70,00 & 80,00 \\
\hline $\begin{array}{r}\text { gemessene } \\
\text { Probentemperatur in }{ }^{\circ} \mathrm{C}\end{array}$ & 19,79 & 29,59 & 39,41 & 48,93 & 58,84 & 68,83 & 78,74 \\
\hline \multicolumn{8}{|l|}{ Mehl M45 F76 } \\
\hline $\begin{array}{r}\text { am Thermostat eingestellte } \\
\text { Temperatur in }{ }^{\circ} \mathrm{C}\end{array}$ & 20,00 & 30,00 & 40,00 & 50,00 & 60,00 & 70,00 & 80,00 \\
\hline $\begin{array}{r}\text { gemessene } \\
\text { Probentemperatur in }{ }^{\circ} \mathrm{C}\end{array}$ & 19,83 & 29,64 & 39,42 & 49,20 & 58,98 & 68,84 & 78,54 \\
\hline \multicolumn{8}{|l|}{ Mehl M45 H2O } \\
\hline $\begin{array}{r}\text { am Thermostat eingestellte } \\
\text { Temperatur in }{ }^{\circ} \mathrm{C}\end{array}$ & 20,00 & 30,00 & 40,00 & 50,00 & 60,00 & 70,00 & 80,00 \\
\hline $\begin{array}{r}\text { gemessene } \\
\text { Probentemperatur in }{ }^{\circ} \mathrm{C}\end{array}$ & 19,93 & 29,54 & 39,44 & 49,30 & 59,05 & 68,89 & 78,55 \\
\hline
\end{tabular}





\section{Abbildungsverzeichnis}

2.1 Übersicht Feuchtemessverfahren . . . . . . . . . . . . . . . . . . . . . . . 4

2.2 Sorptionsisothermen von Lebensmitteln . . . . . . . . . . . . . . . . 6

2.3 Wasserbindungsarten . . . . . . . . . . . . . . . 8

2.4 Geometrie und Elektronegativität des Wassermoleküls . . . . . . . . . 9

2.5 Vereinfachtes Phasendiagramm Wasser . . . . . . . . . . . . . . . 10

2.6 Wasserclusterbildung . . . . . . . . . . . . . . . . . 11

2.7 Potential harmonischer Oszillator . . . . . . . . . . . . . . . 12

2.8 Graphische Darstellung Boltzmannverteilung . . . . . . . . . . . . . . 14

2.9 Potential anharmonischer Oszillator . . . . . . . . . . . . . . . 15

2.10 Energieniveaus harmonischer und anharmonischer Oszillator . . . . . 16

2.11 Normalschwingungen $\mathrm{H}_{2} \mathrm{O}$. . . . . . . . . . . . . . . . . . . 17

2.12 Schwingungsenergieniveaus der OH-Bindung . . . . . . . . . . . . 18

2.13 Schwingungsenergieniveaus des Wassermoleküls in flüssiger Phase . . 20

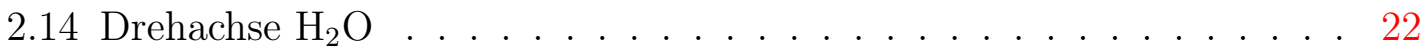

3.1 Trockenschrankmethode, Gerätedarstellung und Prinzipskizze . . . . 27

3.2 MA45 MA100 . . . . . . . . . . . . . . . . . . 28

3.3 WDS $400 \ldots \ldots \ldots \ldots \ldots \ldots$

3.4 Spektroskopie Transmissionsanordnung . . . . . . . . . . . . . . . . . 32

3.5 Spektroskopie Reflexionsanordnung . . . . . . . . . . . . . . . . . 33

3.6 Absorbanzspektren von Wasser und Mehl . . . . . . . . . . . . . . . . 34

3.7 Schema temperierbare Küvette für Transmissionsmessungen . . . . . 36

3.8 Temperaturabhängige Transmissionsspektren von Wasser . . . . . . . 38

3.9 Versuchsaufbau Temperiereinheit . . . . . . . . . . . . . . . . . 39

3.10 Temperaturabhängige Spektren von Mehl M12 . . . . . . . . . . . . . . 40

3.11 Spektrum und Foto von Weizenmehl Type 550 . . . . . . . . . . . . . 42

4.1 Darstellung Transformation PLS . . . . . . . . . . . . . . . . . 50

4.2 Blockschema Faktorisierung beim PLS . . . . . . . . . . . . . . 51

4.3 Over- und Underfitting beim PLS . . . . . . . . . . . . . . . 52

4.4 Kreuz- und Testsetvalidierung . . . . . . . . . . . . . . . . . . 54

4.5 Euklidische Distanz . . . . . . . . . . . . . . . . . . . 58

4.6 Graphische Darstellung des Einflusses von $x$ - und $y$-Ausreißern . . . . 59

4.7 Graphische Darstellung des Hebels (leverage) . . . . . . . . . . . . . . 61 
4.8 Ausreißer in den Referenzwertresiduen . . . . . . . . . . . . . . 63

4.9 Ausreißer in den spektralen Residuen . . . . . . . . . . . . . . . . . 64

4.10 Darstellung linearer Offset . . . . . . . . . . . . . . . . . . 66

4.11 Min-Max-Normierung . . . . . . . . . . . . . . . . . . 67

4.12 Multiplikative Streukorrektur MSC . . . . . . . . . . . . . . . 68

4.13 Anwendung Standard Normal Variate SNV . . . . . . . . . . . . . . 69

4.14 Savitzky-Golay-Glättung . . . . . . . . . . . . . . . . . . . 69

4.15 Savitzky-Golay-Filter . . . . . . . . . . . . . . . . . . . 70

4.16 Funktionsweise Piecewise Direct Standardization . . . . . . . . . . 72

4.17 Funktionsweise Double Windowed Piecewise Direct Standardization . 74

5.1 Diffuse Reflexion . . . . . . . . . . . . . . . . . . . . 75

5.2 Einfluss Mahlgrade Vorhersage und Spektren von Kaffeepulver . . . . 76

5.3 Quereinfluss Röstgrad der Probe . . . . . . . . . . . . . . . . . . 79

5.4 Skizze: Raumwinkel NIR-Messkopf . . . . . . . . . . . . . . . . . 80

5.5 Quereinfluss Abstand Probe zu Sensorkopf 20 bis $350 \mathrm{~mm}$. . . . . . 81

5.6 RMSECV und RMSEP für unterschiedliche Temperaturen und zugehörige Modelle . . . . . . . . . . . . . 86

6.1 Zusammenhang RMSEP und Steigung der Vorhersage bei veränderlicher Temperatur . . . . . . . . . . . . . . . . . 9 90

6.2 Steigung der linearen Regression der Vorhersage bei unterschiedlichen Temperaturen und allen Mehlproben . . . . . . . . . . . . . . . 92

6.3 Vorhersagefehler RMSEP bei unterschiedlichen Temperaturen und allen Mehlproben ..................... . . 93

6.4 Veranschaulichung Bias and Skew adjustment . . . . . . . . . . . 94

7.1 Transferalgorithmus Temperaturkorrektur . . . . . . . . . . . 106

7.2 Spektrenvergleich bei DWPDS von $20^{\circ} \mathrm{C}$ nach $80^{\circ} \mathrm{C} \ldots . . . . . .107$ 


\section{Literaturverzeichnis}

[1] C. Connolly, NIR spectroscopy for foodstuff monitoring, Sensor Review 25(3), 192, 2005, publisher: Emerald Group Publishing Limited.

[2] D. Filmore, Keeping an (infrared) eye out: near-IR spectroscopy is helping pharma move to on-line monitoring of dosage unit production., Modern Drug Discovery 6, 35, 2003, URL http://pubs.acs.org/subscribe/journals/ mdd/v06/i09/pdf/903filmore.pdf.

[3] C. Kurowski, D. Timm, U. Grummisch, U. Meyhack, H. Grunewald, The benefits of near infrared analysis for food product quality., Journal of Near Infrared Spectroscopy 6, A343, 1998.

[4] A. Bozkurt, A. Rosen, H. Rosen, B. Onaral, A portable near infrared spectroscopy system for bedside monitoring of newborn brain, BioMedical Engineering OnLine 4(29), 2005, URL http: / /www . pubmedcentral . nih.gov/ articlerender.fcgi?artid=1112605.

[5] K. A. BAkeEv, Molecular Spectroscopy Workbench, NearInfrared Spectroscopy as a Process Analytical Tool, Part II: At-line and On-line Applications and Implementation Strategies, Spectroscopy 19(1), 39, 2004.

[6] T. Woodcock, G. Downey, C. P. O'Donnell, Review: Better quality food and beverages: the role of near infrared spectroscopy, Journal of Near Infrared Spectroscopy 19(1), 1, 2008.

[7] H. Forcinio, Pharmaceutical Industry Embraces NIR Technology, Spectrocopy 18(9), 16, 2003.

[8] J. Gerold, Auf die Schnelle, Rohstoffidentifikation in der Kosmetikindustrie mit Hilfe der NIR-Spektroskopie, Pharma + Food 5, 46, 2001.

[9] M. C. Pasikatan, J. L. Steele, C. K. Spillman, E. Haque, Review - Near infrared reflectance spectroscopy for online particle size analysis of powders and ground materials, J. Near Infrared Spectrosc. 9, 153, 2001.

[10] O. Berntsson, G. Zackrisson, G. Östling, Determination of moisture in hard gelatin capsules using near-infrared spectroscopy: applications to at-line process control of pharmaceutics, J. Pharm. Biomed. Anal. 15(7), 895, 1997. 
[11] J. E. Mullins, T. V. S. Tomer, Seema, R. Vivilecchia, Moisture determination in powder and tablets by near infrared spectroscopy and its correlation with coulometric Karl Fischer titration analysis, G.I.T. Laboratory Journal 04, $28,2005$.

[12] L. Sukowski, M. Ulmschneider, Inline process analytical technology based on qualitative nearinfrared spectroscopy modeling, Pharm. Ind. 67(7), 830, 2005 .

[13] E. K. AALJOKI, Near infrared spectroscopy finds new roles in process analysis, Kemia - Kemi 22(5), 397, 1995.

[14] I. B. Benson, Not just moisture: a review of some commercially successful near infrared applications., Near Infrared Spectroscopy: The Future Waves, Proceedings of the International Conference on Near Infrared Spectroscopy, 7th, Montreal, Aug. 6-11, 1995 239-248, 1996.

[15] S. Cooke, H.-P. Gut, J. Sellors, J. Oelichmann, Application of FT nearIR spectroscopy in food and drink industries, Lebensmittel- $\&$ Biotechnologie 15(2), 63, 1998.

[16] H. NAGEL, Thermogravimetrische Materialfeuchtebestimmung: Grundlagen und Praktische Anwendungen, Verlag Moderne Industrie, Landsberg/ Lech, 2002 .

[17] C. Blazquez, G. Downey, C. O’Donnell, D. O'Callaghan, V. HoWARD, Prediction of moisture, fat, and inorganic salts in processed cheese by near infrared reflectance spectroscopy and multivariate data analysis., Journal of Near Infrared Spectroscopy 12(3), 149, 2004.

[18] A. Dunko, A. Dovletoglou, Moisture assay of an antifungal by nearinfrared diffuse reflectance spectroscopy, J. Pharm. Biomed. Anal. 28(1), 154, 2002 .

[19] K. A. Bakeev, Molecular Spectroscopy Workbench, NearInfrared Spectroscopy as a Process Analytical Tool, Part I: Laboratory Applications, Spectroscopy 18(11), 32, 2003.

[20] T. Azzouz, A. Puigdomenech, M. Aragay, R. Tauler, Comparison between different data pretreatment methods in the analysis of forage samples using nearinfrared diffuse reflectance spectroscopy and partial leastsquares multivariate calibration method., Analytica Chimica Acta 484(1), 121, 2003, URL http://www.sciencedirect.com/science/article/ B6TF4-48CFRXJ-5/2/d7e3bbfdf7b0306168b4a307bf4eaffb. 
[21] A. Candolfi, R. De Maesschalck, D. Jouan-Rimbaud, P. A. Hailey, D. L. MASSART, The influence of data pre-processing in the pattern recognition of excipients near-infrared spectra., Journal of Pharmaceutical and Biomedical Analysis 21(1), 115, 1999, URL http://www.sciencedirect.com/science/ article/B6TGX-3XFTGOP-F/2/f9be9d33ed48a62acfe9e77752688374.

[22] M. Blanco, J. Coello, H. Iturriaga, S. Maspoch, J. Pages, NIR calibration in non-linear systems: different PLS approaches and artificial neural networks, Chemometrics and Intelligent Laboratory Systems 50(1), 75, 2000, URL http://www.sciencedirect.com/science/article/ B6TFP-3Y8VGYK-7/2/73e6860ace92b4c69e19591ea70997e1.

[23] F. Wülfert, W. KoK, A. Smilde, Influence of Temperature on Vibrational Spectra and Consequences for the Predictive Ability of Multivariate Models, Anal. Chem. 70(9), 1761, 1998, URL http://dx.doi.org/10.1021/ ac9709920.

[24] T. Næs, T. Isaksson, T. Fearn, T. Davies, Multivariate Calibration and Classification, NIR Publications, 2004.

[25] F. Wülfert, W. T. Kok, O. E. de Noord, A. K. Smilde, Linear techniques to correct for temperature-induced spectral variation in multivariate calibration, Chemometrics and Intelligent Laboratory Systems 51(2), 189, 2000, URL http://www.sciencedirect.com/science/article/ B6TFP-40PGW3S-4/2/de3a1d4e66045de479e9e8c34ec48825.

[26] K. Kupfer, Materialfeuchtemessung: Grundlagen, Messverfahren, Applikationen, Normen, expert-Verlag, 1997.

[27] R. Wernecke, Industrielle Feuchtemessung - Grundlagen, Messmethoden, technische Anwendungen, Wiley-VCH Verlag GmbH \& KGaA, Weinheim, 2003.

[28] K. Kupfer (Herausgeber), Electromagnetic Aquametry - Electromagnetic Wave Interaction with Water and Moist Substances, Springer, 2005.

[29] P. W. Atkins, J. De Paula, Physikalische Chemie, Wiley-VCH, 2006.

[30] V. Hopp, Wasser, Wiley-VCH, 2004.

[31] A. Holleman, E. WiBerG, Lehrbuch der organischen Chemie, Walter de Gruyter, 1985.

[32] C. E. Mortimer, Chemie: das Basiswissen der Chemie, Georg Thieme Verlag, Stuttgart, 1996. 
[33] G. WedLeR, Lehrbuch der Physikalischen Chemie, VCH, Weinheim, 3. Auflage, 2004.

[34] J. M. Hollas, Moderne Methoden in der Spektroskopie, Friedr. Vieweg und Sohn Verlagsgesellschaft mbH, Braunschweig, 1995.

[35] D. Eisenberg, W. Kauzmann, The structure and properties of water, Oxford University Press, London, 1969.

[36] S. Y. Venyaminov, F. G. Prendergast, Water (H2O and D2O) Molar Absorptivity in the 1000-4000 Range and Quantitative Infrared Spectroscopy of Aqueous Solutions, Analytical Biochemistry 248(2), 234, 1997.

[37] F. Bartha, O. Kapuy, C. Kozmutza, C. Van Alsenoy, Analysis of weakly bound structures: hydrogen bond and the electron density in a water dimer, Journal of Molecular Structure 666-67, 117, 2003.

[38] M. Chaplin, Hydrogen Bonding in Water, http://www.Isbu.ac.uk/water/ hbond.html, 2004.

[39] R. C. Dougherty, Temperature and pressure dependence of hydrogen bond strength: A perturbation molecular orbital approach, Journal of Chemical Physics 109(17), 7372, 1998.

[40] American Society for Testing and Materials international, Standard Practices for Infrared Multivariate Quantitative Analysis, ASTM-Norm, 2005, ASTM 1655-05.

[41] Deutsches Institut für Normung, Bestimmung des Feuchtegehaltes von Getreide und Getreideerzeugnissen, DIN-Norm, 1967, DIN 10350.

[42] H. StÖCKER, Taschenbuch der Physik, Band 4, Verlag Harri Deutsch, 2000.

[43] Sartorius AG, Göttingen, Betriebsanleitung Sartorius WDS 400, Coulometer zur Wassergehaltsbestimmung, 2004.

[44] W. Schmidt, Optische Spektroskopie, Wiley-VCH Verlag GmbH, Weinheim, 2000 .

[45] G. Kortüm, Reflexionsspektroskopie, Springer-Verlag, Berlin, 1969.

[46] W. Gottwald, G. Wachter, IR-Spektroskopie für Anwender, Wiley VCH, Weinheim [u.a.], 1997.

[47] W. F. MCClure, Near-Infrared Spectroscopy: The Giant is Running Strong, Anal. Chem. 66(1), 43A, 1994. 
[48] J. J. Workman, Review of Process and Non-invasive Near-Infrared and Infrared Spectroscopy: 1993-1999, Appl. Spec. Rev. 34(1\& 2), 1, 1999.

[49] P. Williams, K. H. NorRis (Herausgeber), Near-infrared technology in the agricultural and food industries, St. Paul, Minn.: American Association of Cereal Chemists, 2 Auflage, 2001.

[50] E. Wust, In-Line Measurement of High Moisture Products, Near-infrared Spectroscop.: Future Waves 7, 268, 1996.

[51] E. Cleve, E. Bach, E. Schollmeyer, Using chemometric methods and NIR spectrophotometry in the textile industry, Anal. Chim. Act. 420(2), 163, 2000.

[52] A. Fong, G. M. Hieftue, Near-Infrared Measurements of Relative and Absolute Humidity through Detection of Water Adsorbed on a Silica Gel Layer, Anal. Chem. 67(6), 1139, 1995.

[53] M. Chaplin, Molecular Vibration and Absorption of Water Molecules, http: //www.martin.chaplin.btinternet.co.uk/vibrat.html, 2004.

[54] F. D. BarbozA, R. J. Poppi, Determination of alcohol content in beverages using short-wave near-infrared spectroscopy and temperature correction by transfer calibration procedures, Analytical and Bioanalytical Chemistry 377(4), 695, 2003, URL http://www.springerlink.com/openurl. asp?genre=article\&id=doi:10.1007/s00216-003-2128-2.

[55] H. Kellerhans, Spectrometer with automatic referencing, Patentschrift, 2002, URL http://www.freepatentsonline.com/EP1221597A1.html, EP1221597.

[56] S. Gross, Machbarkeitsstudie für einen optogravimetrischen Materialfeuchteschnellbestimmer zur Feuchtebestimmung von Schüttgütern, Diplomarbeit, Hochschule für Angewandte Wissenschaft, Fachhochschule Hildesheim/Holzminden/Göttingen, Fakultät für Naturwissenschaften und Technik, Göttingen, 2004.

[57] Galactic, Galactic Universal Data Format Specification, 1997, URL http: //www. thermo.com/grams.

[58] Eigenvector Research, InC., DataSet - Standard Data Object, for use with MATLAB, 2005, URL http://www.eigenvector.com/.

[59] H. P. Latscha, G. Schilling, H. A. Klein, Chemiedatensammlung: Laborhilfen für Studium und Praxis, Band 246, Springerverlag, Berlin, 1990. 
[60] M. Отто, Chemometrics, Statistics and Computer Application in Analytical Chemistry, Wiley-VCH, 2007.

[61] L. Thygesen, S.-O. Lundqvist, NIR measurement of moisture content in wood under unstable temperature conditions. Part 1. Thermal effects in near infrared spectra of wood, J. Near Infrared Spectrosc. 8(3), 183, 2000.

[62] Y.-A. Woo, J.-W. Ahn, I.-K. Chun, H.-J. Kim, Development of a Method for the Determination of Human Skin Moisture Using a Portable Near-Infrared System, Anal. Chem. 73(20), 4964, 2001.

[63] V. J. Frost, Kalibrationsoptimierung mittels genetischer Algorithmen: Eine Methode zur automatischen Selektion von PCR-Faktoren in der NIRSpektrometrie, Dissertation, Gerhard-Mercator-Universität - Duisburg, 2000.

[64] E. R. Malinowski, D. G. Howery, Factor Analysis in Chemistry, John Wiley and Sons, New York, 1980.

[65] F. ZEYEN, NIR-Spektrometrie als Methode der quantitativen Analyse von synthetischen und pflanzlichen Wirkstoffen in Tabletten und Granulaten, Dissertation, Gerhard-Mercator-Universität-Gesamthochschule - Duisburg, 2000.

[66] M. Stone, Cross-validatory choice and assessment of statistical prediction, Journal of the Royal Statistical Society: Series B 39, 111, 1974.

[67] J.-P. Conzen, Multivariate Kalibration, Bruker Optik GmbH, 2001.

[68] R. G. Whitfield, M. E. Gerger, R. L. Sharp, Near-Infrared Spectrum Qualification via Mahalanobis Distance Determination, Applied Spectroscopy 41(7), 1204, 1987.

[69] R. D. Cook, S. Weisberg, Residuals and Influence in Regression, Chapman and Hall, New York, 1982.

[70] P. F. Velleman, R. E. Welsch, Efficient Computing of Regression Diagnostics, American Statist 35, 234, 1981.

[71] H. Martens, T. NÆs, Multivariate Calibration, John Wiley \& Sons, New York, 1998.

[72] P. Geladi, D. MacDougall, H. Martens, Linearization and Scatter-Correction for Near-Infrared Reflectance Spectra of Meat, Applied Spectroscopy 39, $377,1985$.

[73] A. Savitzky, M. J. E. Golay, Smoothing and Differentiation of Data by Simplified Least Squares Procedures, Anal. Chem. 36(8), 1627, 1964. 
[74] H. Siesler, Y. Ozaki, S. Kawata, H. Heise, Near-Infrared Spectroscopy, principles, instruments, applications, Wiley-VCH, 2002.

[75] B. Walczak, E. Bouveresse, D. Massart, Standardization of Near-Infrared Spectra in the Waveletdomain, Chemometr. Intell. Lab. 36, 41, 1997.

[76] J. S. Shenk, M. O. Westerhaus, J. Templeton, W. C., Calibration Transfer Between near Infrared Reflectance Spectrophotometers, Crop Sci 25(1), 159, 1985, URL http://crop.scijournals.org/cgi/content/ abstract/cropsci;25/1/159.

[77] S. Wold, H. Antti, F. Lindgren, J. Öhman, Orthogonal signal correction of near-infrared spectra, Chemometr. Intell. Lab. 44, 175, 1998.

[78] Y. Wang, D. J. Veltkamp, B. R. Kowalski, Multivariate Instrument Standardization, Analytical Chemistry 63, 2750, 1991.

[79] Y. WANG, M. J. LySAGHt, B. R. Kowalski, Improvement of multivariate calibration through instrument standardization, Analytical Chemistry 64(5), 562, 1992, URL http://pubs.acs.org/doi/abs/10.1021/ac00029a021.

[80] Z. Wang, T. Isaksson, B. R. Kowalski, New approach for distance measurement in locally weighted regression, Analytical Chemistry 66(2), 249, 1994, URL http://pubs.acs.org/doi/abs/10.1021/ac00074a012.

[81] Z. Wang, T. Dean, B. R. Kowalski, Additive Background Correction in Multivariate Instrument Standardization, Analytical Chemistry 67(14), 2379, 1995, URL http://pubs .acs.org/doi/abs/10.1021/ac00110a009.

[82] P. Kubelka, F. Munk, Ein Beitrag zur Optik der Farbanstriche, Z. Techn. Physik 12, 593, 1931.

[83] L. C. Maillard, Réaction générale des acides aminés sur les sucres, Journal de Physiologie 14, 813, 1912.

[84] L. C. Maillard, Action des acides aminés sur les sucres: formation des mélanoïdines par voie méthodique, Compte-rendu de l'Académie des sciences (154), 66, 1912.

[85] L. C. Maillard, Réaction générale des acides aminés sur les sucres: ses conséquences biologiques, Compte-rendu de la Société de Biologie (72), 599, 1912.

[86] H. Kuchling, Nachschlagebücher Für Grundlagenfächer, Physik, VEB Fachbuchverlag Leipzig, Leipzig, 1985. 
[87] P. Apian-Bennewitz, Messung und Modellierung von Lichtstreuenden Materialien zur Computer-Simulation von Tageslichtbeleuchtung, Dissertation, Albert-Ludwigs-Universität, Freiburg im Breisgau, 1995.

[88] J. Hageman, J. A. Westerhuis, A. Smilde, Temperature robust multivariate calibration: An overview of methods for dealing with temperature influences on near infrared spectra, J. Near Infrared Spectrosc. 13, 53, 2005.

[89] F. Chauchard, J. M. Roger, V. Bellon-Maurel, Correction of the temperature effect on near infrared calibration - Application to soluble solid content prediction., Journal of Near Infrared Spectroscopy 12, 199, 2004.

[90] K. Diedrich, H. Nagel, W. Spannagel, Trocknungswaage, Patentschrift, 2006, DE 102004053734 B4.

[91] C. Merkwirth, U. Parlitz, W. Lauterborn, Fast Exact and Approximate Nearest Neighbor Searching for Nonlinear Signal Processing, Phys. Rev. E 62(2), 2089, 2000.

[92] D. Engster, U. Parlitz, Handbook of Time Series Analysis, Kapitel 3 Local and Cluster Weighted Modeling for Time Series Prediction, WILEY-VCH Verlag, 2006.

[93] U. PARLitz, Identification of pre-sliding friction dynamics, Chaos 14(2), 420, 2004. 


\section{Danksagung}

Ich möchte Herrn Prof. Lauterborn für die Betreuung meiner Arbeit als Referent seitens der Universität Göttingen danken.

Stellvertretend für die Mitarbeiter des Laser Laboratoriums Göttingen e.V. danke ich Herrn Prof. Marowsky für die Möglichkeit der Durchführung der experimentellen und theoretischen Untersuchungen im Rahmen dieser Arbeit. Den Mitarbeitern des Laser Laboratoriums Göttingen e.V. danke ich für die Betreuung und ihre Hilfestellungen bei Fragen und Problemen.

Herrn Dr. Christoph Lenth danke ich für die Betreuung meiner Arbeit von Seite des Laboratoriums Göttingen e.V..

Für die Sartorius AG möchte ich Herrn Dr. Manfred Rahe für die interessante Themenstellung, die Bereitstellung der Mittel und die Unterstützung während der Arbeit danken.

Besonders danke ich auch der Gruppe unter der Leitung von Herrn Spannagel für die Betreuung seitens der Sartorius AG und ihre fortwährende Hilfsbereitschaft.

Ein besonderer Dank gilt Herrn Dr. Christian Grimm für die stete Diskussionsbereitschaft, Betreuung und Korrektur meiner Arbeit und für seine Tipps zur Formatierung. Außerdem danke ich ihm für seinen Support in IATEX.

Für die Anfertigung der mechanischen Teile und der Umbauten an den Versuchsaufbauten danke ich der Ausbildungswerkstatt der Sartorius AG, der Versuchswerkstatt der Mechatronik in Vertretung Herrn Brandenburg und Herrn Andreas Sparta vom Laser Laboratorium Göttingen e.V..

Ein weiterer Dank geht an Herrn Christian Salb vom Laser Laboratorium Göttingen e.V. für die Unterstützung bei der Anfertigung des Versuchsaufbaus zur Reflektionsmessung und für die Unterstützung bei den experimentellen Untersuchungen.

Herrn Björn Helmker möchte ich für das Korrekturlesen dieser Arbeit in Hinsicht auf Rechtschreib- und Grammatikfehler danken.

Ganz besonders danke ich meinen Eltern und meiner Familie, die mich während der Dissertationsphase mit ihrer Hilfe und ihrem Verständnis stets unterstützt haben. Ich danke Ihnen für die seelische und moralische Unterstützung während der gesamten Zeit, für ihre Liebe und ihr Verständnis, die das Gelingen dieser Arbeit erst möglich machten.

Meiner Tanzpartnerin Frau Martina Lotsch möchte ich an dieser Stelle einen besonderen Dank für die stete und flexible Bereitschaft, den Trainingsplan für die Turniere, Meisterschaften und auch das Kadertraining des Landes Niedersachsen immer wieder an meinen Zeitplan der Dissertationsphase anzupassen. Außerdem 
möchte ich Ihr für Ihr Verständnis und die seelische und moralische Unterstützung in der Endphase der Arbeit danken. Das Training hat mir die nötige Abwechslung und Erholung ermöglicht. 


\section{Lebenslauf}

\section{Sven Groß}

geboren $\quad 01$. November 1978 als Sohn von Bernd Groß und Sigrid Groß, geb. Rutz, in Göttingen

Nationalität deutsch

Tätigkeit Doktorand am Laser Laboratorium Göttingen e.V. in Zusammenarbeit mit der Sartorius AG

\section{Hochschultätigkeiten}

2001-2003 Vorbereitung und Durchführung des Einführungstutoriums Mathematik für Erstsemesterstudenten

2001-2004 Mitglied der Studienkommision der Fakultät Naturwissenschaften und Technik, HAWK Göttingen

2001-2004 Mitglied des Praxisverbundbeirates der Fakultät Naturwissenschaften und Technik, HAWK Göttingen

\section{Hochschulausbildung}

seit 2005

2004

2004

$2003-2004$

2002
Doktorarbeit an der an der Universität Göttingen

Diplom mit Auszeichnung als bester Student des Semesters an der HAWK Göttingen

Diplomarbeit am Laser-Laboratorium Göttingen e. V. in Zusammenarbeit mit der Sartorius AG Göttingen, Schwerpunkt: „Spektroskopische Untersuchungen an Schüttgütern aus Lebensmittelindustrie, Chemie und Pharmazie"

Studienarbeit „Bestimmung des gesamten organischen Kohlenstoffgehalts (TOC) mittels laserinduzierter Fluoreszenz" am Laser-Laboratorium Göttingen e. V.

Studienarbeit „Auswertung und Darstellung eines Temperaturfeldes" in Visual $\mathrm{C}++$ incl. Windowsoberflächenprogrammierung 

Hochschule für angewandte Wissenschaft und Kunst, Fakultät Naturwissenschaft und Technik, Göttingen

\begin{tabular}{|c|c|}
\hline \multicolumn{2}{|r|}{ Schulbildung } \\
\hline 1998 & Abitur (Leistungskurse Mathematik und Physik) \\
\hline $1991-1998$ & Felix-Klein-Gymnasium in Göttingen \\
\hline $1988-1991$ & Orientierungsstufe Jahnschule in Göttingen \\
\hline $1985-1988$ & Grundschule in Diemarden \\
\hline \multicolumn{2}{|r|}{ Zivildienst } \\
\hline 1998-1999 & Zivildienst in der Multiple-Sklerose-Beratungsstelle in Göttingen \\
\hline \multicolumn{2}{|r|}{ Zusätzliche Ausbildungen } \\
\hline $1999-2002$ & Ausbildung zum Industrieelektroniker mit Facharbeiterabschluss \\
\hline 2004 & $\begin{array}{l}\text { Ausbildung zum Laserschutzbeauftragten an der HAWK Fakul- } \\
\text { tät Naturwissenschaften und Technik in Göttingen }\end{array}$ \\
\hline \multicolumn{2}{|r|}{ Ehrenamtliches Engagement } \\
\hline $1999-2002$ & $\begin{array}{l}\text { Trainer / Co-Trainer Standardformationen (2. Team und Kin- } \\
\text { dergruppe) des TSC-Schwarz-Gold-Göttingen e. V. }\end{array}$ \\
\hline $2005-2008$ & $\begin{array}{l}\text { Trainer Tanzkreis Erwachsene und Senioren des TSC-Schwarz- } \\
\text { Gold-Göttingen e. V. }\end{array}$ \\
\hline seit 2008 & Mitglied im Vorstand des TSC-Schwarz-Gold-Göttingen e. V. \\
\hline seit 2009 & $\begin{array}{l}\text { Vorsitzender des geschäftsführenden Vorstandes des TSC-Schwarz- } \\
\text { Gold-Göttingen e. V. }\end{array}$ \\
\hline
\end{tabular}

